NUREG/CR-1498

PNL-3324

\title{
Population Dose Commitments Due to Radioactive Releases from Nuclear Power Plant Sites in 1977
}

Prepared by D. A. Baker

Pacific Northwest Laboratory

Operated by

Battelle Memorial Institute

Prepared for

U.S. Nuclear Regulatory

Commission 


\section{NOTICE}

This report was prepared as an account of work sponsored by an agency of the United States Government. Neither the United States Government nor any agency thereof, or any of their employees, makes any warranty, expressed or implied, or assumes any legal liability or responsibility for any third party's use, or the results of such use, of any information, apparatus product or process disclosed in this report, or represents that its use by such third party would not infringe privately owned rights.

Available from

GPO Sales Program

Division of Technical Information and Document Control

U. S. Nuclear Regulatory Commission

Washington, D. C. 20555

and

National Technical Information Service

Springfield, Virginia 22161 
NUREG/CR-1498

PNL-3324

\section{Population Dose Commitments Due to Radioactive Releases from Nuclear Power Plant Sites in 1977}

Manuscript Completed: August 1980

Date Published: October 1980

Prepared by

D. A. Baker

Pacific Northwest Laboratory

Richland, WA 99352

Prepared for

Division of Technical Support

Office of Management and Program Analysis

U.S. Nuclear Regulatory Commission

Washington, D.C. 20555

NRC FIN No. B2243 



\section{ABSTRACT}

Population radiation dose commitments have been estimated from reported radionuclide releases from commercial power reactors operating during 1977 . Fifty-year dose commitments from a one-year exposure were calculated from both liquid and atmospheric releases for four population groups (infant, child, teen-ager and adu $3 t$ ) residing between 2 and $80 \mathrm{~km}$ from each site. This report tabulates the results of these calculations, showing the dose comitments for both liquid and airborne pathways for each age group and organ. Also included for each site is a histogram showing the fraction of the total population within 2 to $80 \mathrm{~km}$ around each site receiving various average dose commitments from the airborne pathways.

The total dose commitment from both 1 iquid and airborne pathways ranged from a high of 220 person-rem to a low of 0.003 person-ren with an arithmetic mean of 16 person-rem. The total population dose for all sites was estimated at 700 person-rem for the 92 million people considered at risk.

The average individual dose commitment from all pathways on a site basis ranged from a low of $2 \times 10^{-5} \mathrm{mrem}$ to a high of $0.1 \mathrm{mren}$. No attempt was made in this study to determine the maximum dose comitment received by any one individual from the radionuclides released at any of the sites. 



\section{CONTENTS}

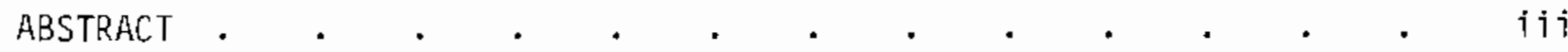

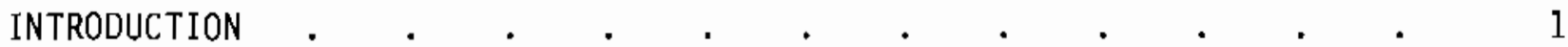

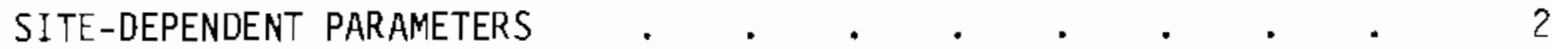

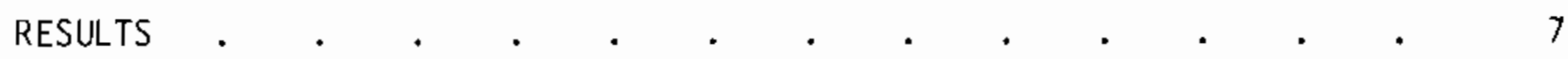

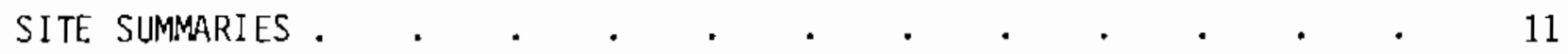

Arkansas . . . . . . . . . . . . . . . . . . 12

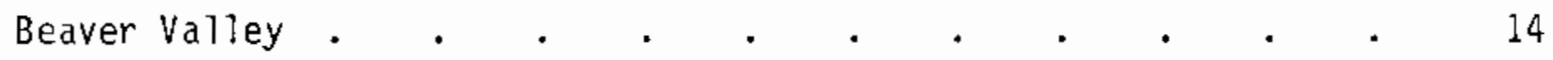

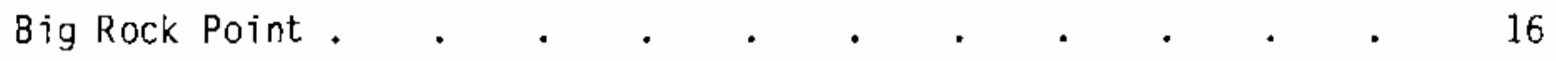

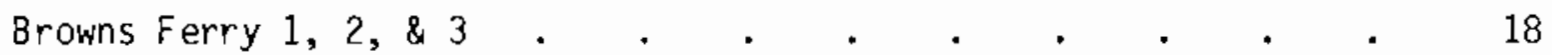

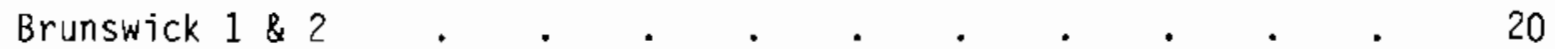

Calvert Cliffs . . . . . . . . . . . . . . . . . 22

Cook . . . . . . . . . . . . . . . . 24

Cooper Station . . . . . . . . . . . . . . . . . 26

Crystal River . $\quad . \quad$. $\quad . \quad$. . . . . . . . . . . 28

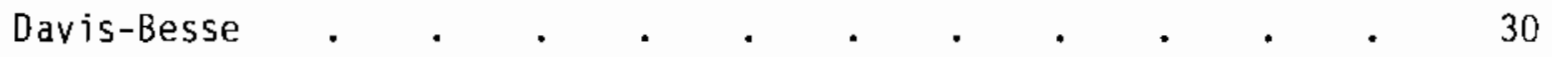

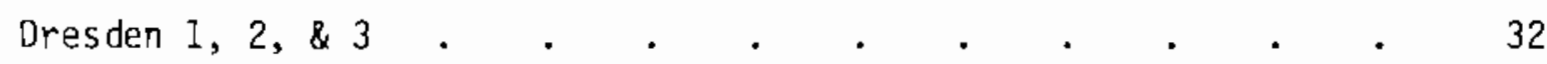

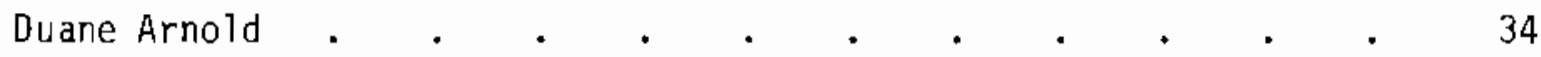

J. A. Fitzpatrick . . . . . . . . . . . . . . . 36

Fort Calhoun . $\quad . \quad$. . . . . . . . . . . . 33

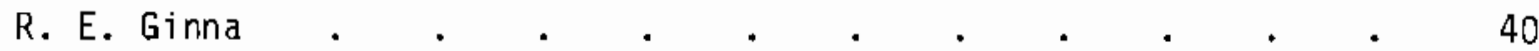

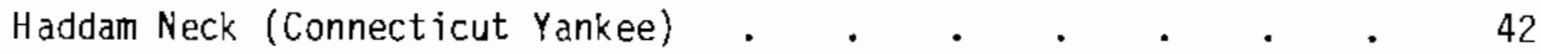

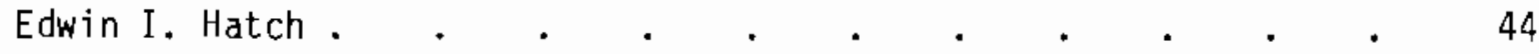

Humboldt Bay . . . . . . . . . . . . . . . . 46

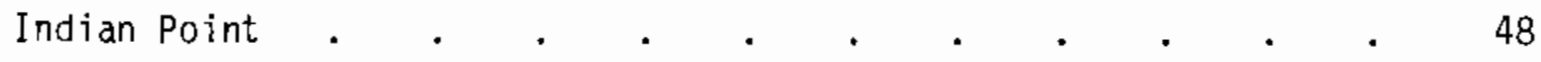




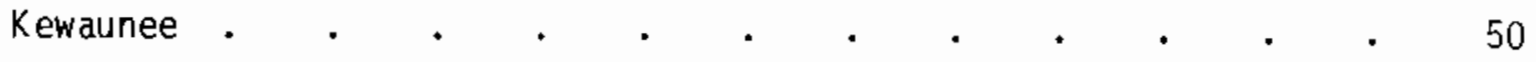

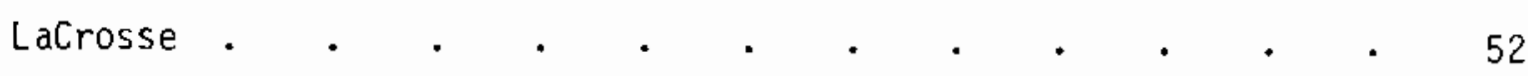

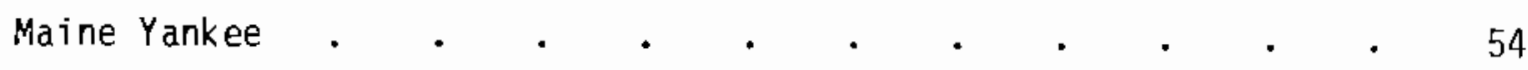

Millstone Point $1 \& 2$ \& . . . . . . . . . . . . 56

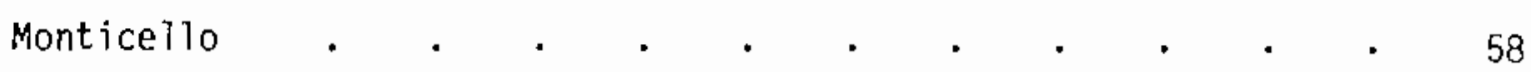

Nine Mile Point $. \quad . \quad . \quad . \quad . \quad . \quad . \quad . \quad . \quad 60$

0conee $1,2, \& 3 \quad . \quad . \quad . \quad . \quad . \quad . \quad . \quad . \quad . \quad . \quad 62$

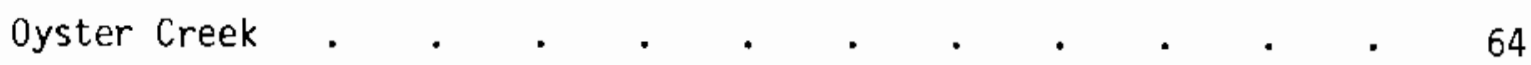

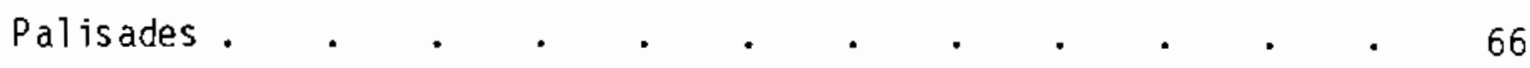

Peach Bottorn $2 \& 3$ \& . . . . . . . . . . . . . . . . 68

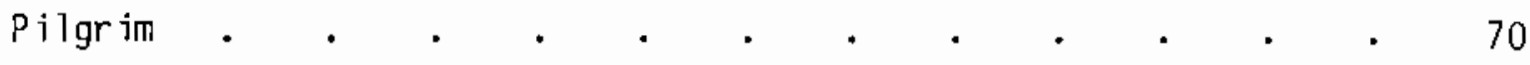

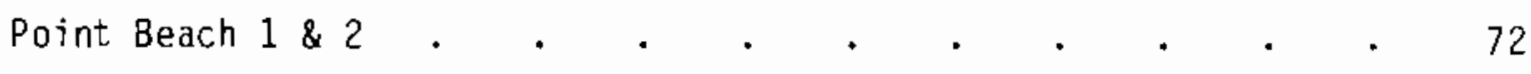

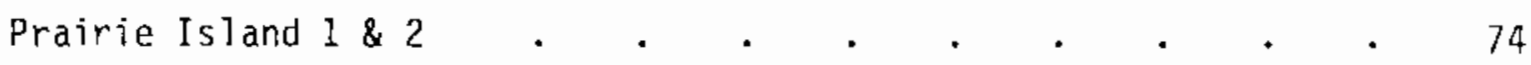

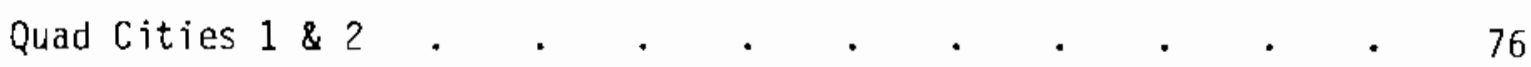

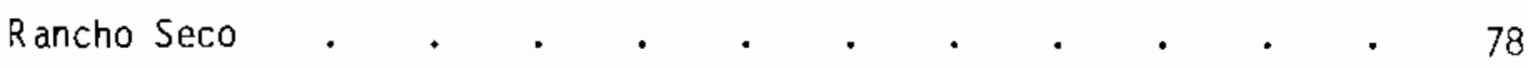

H. B. Robinson . . . . . . . . . . . . . . 80

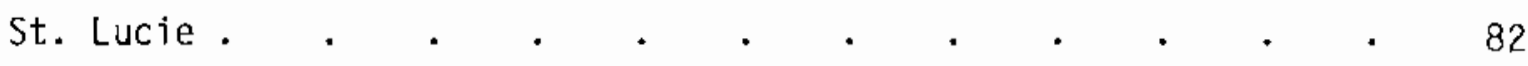

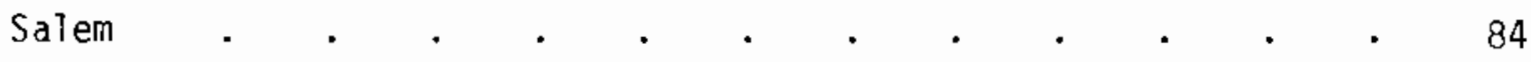

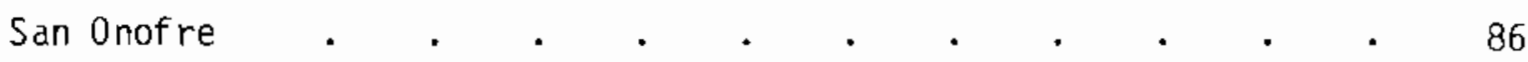

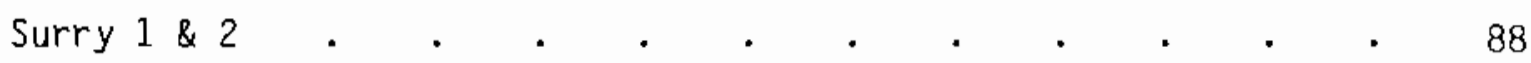

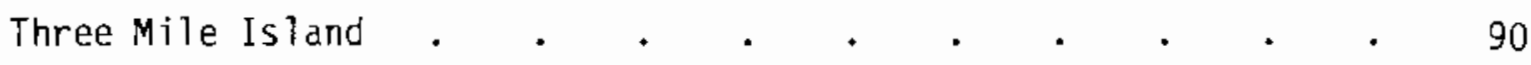

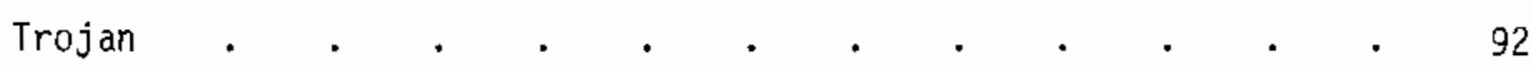

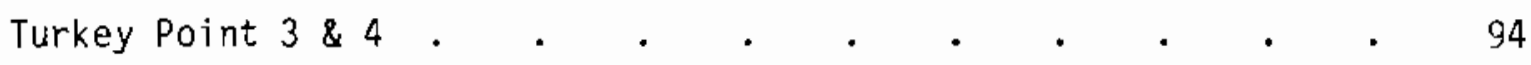

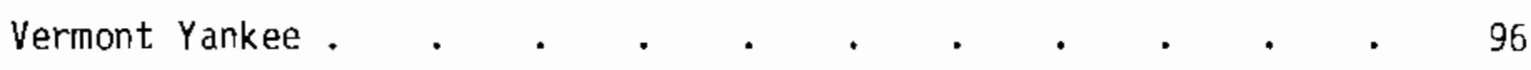

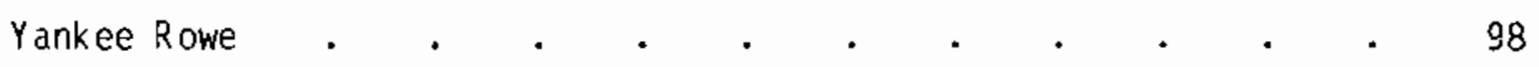




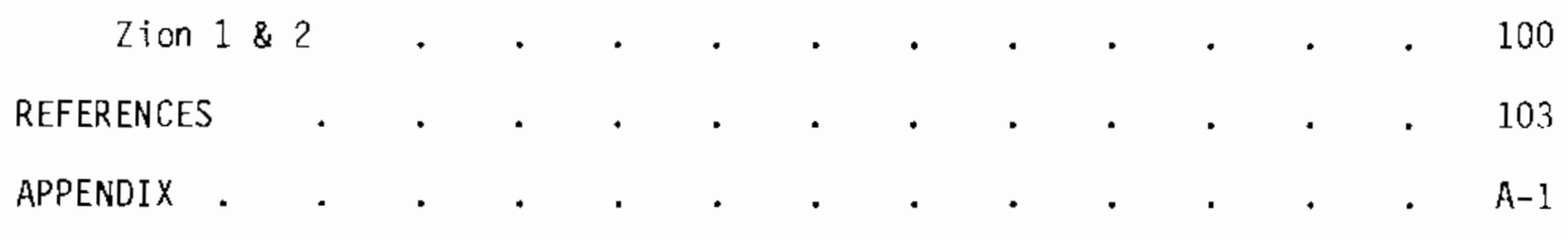



The Pacific Northwest Laboratory (PNL) is operated by Battelle Memorial Institute for the Department of Energy (BOE). This study, sponsored by the Nuclear Regulatory Commission (NRC) and conducted at PNL, estimates radiation dose commitments from reported radionuclide releases at 60 commercial power reactors operating during 1977. This work is a continuation of the study done for 1975 and 1976 releases (Baker, Soldat and Watson 1977, Baker 1979). In this study, as in the last, we estimated the population (collective) dose conmitment (a) from both the liquid and gaseous releases to four age groups making up the population residing in the region of the site: infant ( 0 to 1 $\mathrm{yr}$ ), child (1 to $11 \mathrm{yr}$ ), teen-ager (11 to $17 \mathrm{yr}$ ) and adult (17 $\mathrm{yr}$ and older).

The particular organs of reference in this study are listed in Table 1. The major pathways by which radionuclides travel from the reactor to the individual receptors are shown in Table 2. Other possible liquid pathways

\section{TABLE 1. Organs Considered in This Study}

\section{Organs Affected by Airborne Releases}

Total body

Thyroid

Bone

GI tract

Liver

Lung
Organs Affected by Waterborne Releases

Total body

Thyroid

Bone

GI tract

Liver

TABLE 2. Pathways Considered in This Study by which Radionuclides Travel from Reactors to Persons

Pathways for

Airborne Releases

Air submersion

Contaminated ground

Inhalation

Ingestion of food crops and animal products
Pathways for

Waterborne Releases

Ingestion of drinking water Ingestion of fish and invertebrates

(a) As used in this report, dose commitment describes the total-body dose equivalent received over 50 years from intake during the year 1977. 
such as direct exposure from waterborne activities (swimming, boating, shoreline recreation) and internal exposure through ingestion of food produced using contaminated irrigation water were not included. This was because we have found from past experience that the doses from these pathways is generally much smaller than the doses from the pathways considered in this study.

The regional population for which we estimated doses included those persons estimated to be living in a region between 2 and $80 \mathrm{~km}$ around the reactor sites during 1977. We estimated the numbers of persons by extrapolating Bureau of Census data for 1970 to 1977. Atmospheric transport factors (annual average dilution and annual average deposition) were calculated for the region around each site using appropriate meteorological data supplied by the NRC's Office of Nuclear Reactor Regulation. To calclilate the doses, we used models approved by the NRC. We incorporated these models into two small computer codes to expedite the dose calculations involved for each site.

Site-specific parameters other than releases, meteorology and population were obtained from environmental statements for the various reactors when available (Table 3). Such parameter values include the total population drinking contaminated water, river flow, dilution flow from the reactors (for sites not on rivers), fish and invertebrate harvest for region, and dilution factors for drinking water and aquatic foods.

The reactors included in this study, their type, licensed thermal power rating and net electrical output for 1977 are listed in Table 4 . Those reactors which had an operating augmented gaseous radioactive waste system in 1977 are identifjed in the table. Populations at risk and the dose comitments derived in this study are also tabulated.

\section{SITE-DEPENDENT PARAMETERS}

The section entitled Site Sumaries gives the location (including latitude and Tongitude) for each reactor site and the estimated 1977 population within 2 to $80 \mathrm{~km}$ around the site. This population is derived from the 1970 census by extrapolation to 1977. In addition, the location of major metropolitan centers within $80 \mathrm{~km}$ are listed along with their 1977 extrapolated populations. The populations of the Standard Metropolitan Statistical Areas (SMSA) are given where applicable. Next, the average production rates of vegetable crops and animal products are given for the area within an $80-\mathrm{km}$ radius based up on the statewide average. This production has been reduced for sites on lakes and seacoasts to account for the presence of the body of water. An animal grazing factor is estimated for each site location. This factor accounts for the fraction of the year during which grazing animals such as milk cows and beef cattle graze on fresh pasture in the region around the site. After average production rates are given, the location of the meteorological station used in the acquisition of diffusion climatology data is indicated, along with the percent data recovery and period of record. 


\section{TABLE 3. Environmental Statements for Power Plants Included in This Study}

\begin{tabular}{|c|c|c|c|c|}
\hline $\begin{array}{l}\text { Site } \\
\text { Number }\end{array}$ & Reactor site & $\begin{array}{l}\text { Docket } \\
\text { Number }\end{array}$ & Date & Remarks \\
\hline 1 & Big Rock Point & & & ES(a) not available \\
\hline 2 & Brown's Ferry 1, 2, 3 & & Ju1 71 & $\begin{array}{l}\text { ES published by Tennessee } \\
\text { Valley Authority }\end{array}$ \\
\hline 3 & Cooper Station & $50-298$ & Feb 73 & Draft ES \\
\hline 4 & Dresden 1 & & & ES of Dresden 2, 3 used \\
\hline 4 & Dresden 2, 3 & $50-237,50-249$ & Nov 73 & \\
\hline 5 & Beaver Valley 1 & $50-334$ & Jul 73 & \\
\hline 6 & Humboidt Bay & & & \\
\hline 7 & LaCrosse & $50-409$ & Jun 76 & Draft ES \\
\hline 8 & Millstone Point 1,2 & $50-245,50-336$ & Jun 73 & \\
\hline 9 & Monticello & $50-263$ & Nov 72 & \\
\hline 10 & Nine Mile Point 1 & $50-220$ & $\operatorname{Jan} 74$ & \\
\hline 11 & Dyster Creek & $50-219$ & Dec 74 & \\
\hline 12 & Peach Bottom 2, 3 & $50-277,50-278$ & Apr 73 & \\
\hline 13 & Pilgrim 1 & $50-293$ & May 72 & \\
\hline 14 & Quad Cities 1, 2 & $50-254,50-265$ & Sep 72 & \\
\hline 15 & Vermont Yankee & $50-271$ & Jul 72 & \\
\hline 16 & St. Lucie & $50-335$ & Jun 73 & \\
\hline 17 & Brunswick 1,2 & $50-324,50-325$ & Juก 73 & Draft ES \\
\hline 18 & Duane Arnold & $50-331$ & Mar 73 & \\
\hline 19 & J. A. Fitzpatrick & $50-333$ & Mar 73 & \\
\hline 20 & E. I. Hatch & $50-321$ & $0 \mathrm{ct} 72$ & \\
\hline 21 & Arkansas 1 & $50-313$ & Feb 73 & \\
\hline 22 & $\begin{array}{l}\text { Connect icut Yankee } \\
\text { (Haddam Neck) }\end{array}$ & $50-213$ & oct 73 & \\
\hline 23 & Fort Calhoun & $50-285$ & Aug 72 & \\
\hline 24 & H. 8. Robinson & $50-261$ & Apr 74 & \\
\hline 25 & Indian Point 1,2 & $50-247$ & Sep 72 & ES of Indian Point 2 used \\
\hline 26 & Salem & $50-272,50-311$ & Apr 73 & \\
\hline 27 & Kewaunee & $50-305$ & $\operatorname{Dec} 72$ & \\
\hline 28 & Maine Y ankee & $50-309$ & Ju1 72 & \\
\hline 29 & Oconee $1,2,3$ & $\begin{array}{l}50-269,50-270 \\
50-287\end{array}$ & $\operatorname{Mar} 72$ & \\
\hline 30 & Palisades & $50-255$ & Jun 72 & \\
\hline 31 & Point Beach 1, 2 & $50-266,50-301$ & May 72 & \\
\hline 32 & Prairie island 1,2 & $50-282,50-306$ & May 73 & \\
\hline 33 & R. E. Ginna & $50-244$ & Dec 73 & \\
\hline 34 & San Onofre 1 & $50-206$ & oct 73 & \\
\hline 35 & Surry 1,2 & $50-281$ & Jun 72 & ES of Surry 2 used \\
\hline 36 & Three Mile Is 1 and 1 & $50-289$ & Dec 72 & \\
\hline 37 & Turkey Point 3,4 & $50-250,50-251$ & Feb 72 & Draft ES \\
\hline 38 & Yankee Rowe & & & ES not available \\
\hline 39 & Zion 1,2 & $50-295,50-304$ & Dec 72 & \\
\hline 40 & Calvert Cliffs 1 & $50-317$ & Apr 73 & \\
\hline 41 & Cook 1 & $50-315$ & Aug 73 & \\
\hline 42 & Trojan & $50-344$ & Jan 73 & Draft ES \\
\hline 43 & Rancho Seco & $50-312$ & $\operatorname{Mar} 73$ & \\
\hline 44 & Crystal River $3(b)$ & $50-302$ & May 73 & \\
\hline 45 & Davis-Besse (b) & $50-346$ & Mar 73 & \\
\hline
\end{tabular}

(a) Environmental Statement

(b) Added for 1977 
TABLE_4. Reactor Characteristics and PopuTation Total Body Dose Commitments, 1977

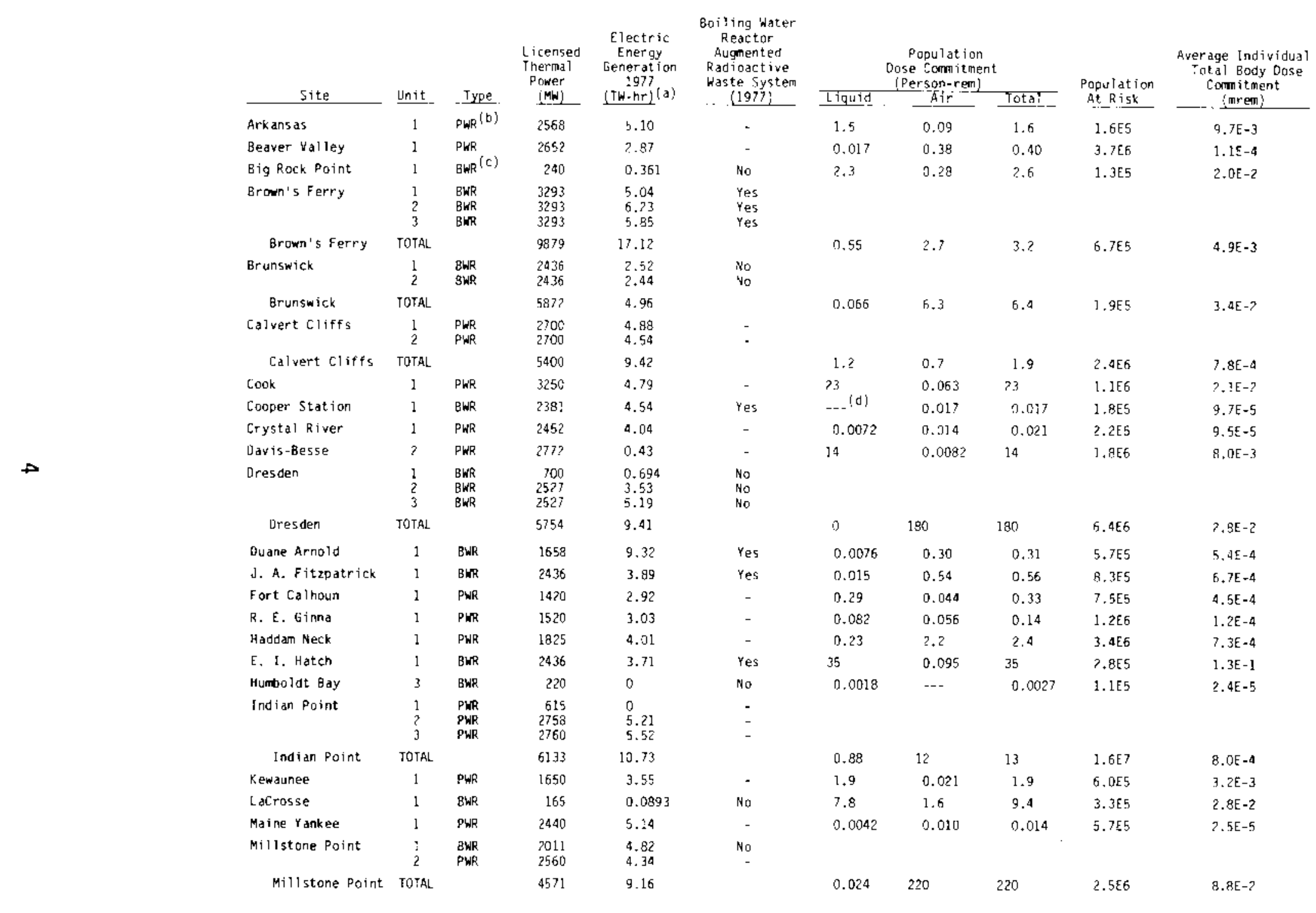


TABLE 4 (contd)

\begin{tabular}{|c|c|c|c|c|c|c|c|c|c|c|}
\hline Manticello & 1 & BWF & 1670 & 3.57 & Yes & 0 & 0.20 & 0.20 & $? .1 E 6$ & $9.6 \mathrm{E}-5$ \\
\hline Nine Mile Point & 1 & BWF & 1850 & 2.95 & No & 3.0 & 0.098 & 3.1 & $8.3 E 5$ & $3.7 E-3$ \\
\hline Oconee & $\begin{array}{l}1 \\
2 \\
3\end{array}$ & $\begin{array}{l}\text { PWF } \\
\text { PWR } \\
\text { PWR }\end{array}$ & $\begin{array}{l}2568 \\
2568 \\
2568\end{array}$ & $\begin{array}{l}3.94 \\
3.83 \\
5.24\end{array}$ & : & & & & & \\
\hline Oconee & TOTAL & & 7704 & $3.0 \%$ & & 37 & 0.69 & 39 & $7.4 \varepsilon 5$ & $5.1 \mathrm{E}-2$ \\
\hline Oyster Creex & 1 & BWR & 5930 & 3.25 & No & 0.011 & 41 & 41 & $3.3 E 6$ & 1.2E-2 \\
\hline Palis ades & $:$ & PWP & 2530 & 5.08 & - & 0.63 & 0.0015 & 0.67 & $1.0 \mathrm{E} 6$ & 6. $7 E-4$ \\
\hline Peach Bottom & ${ }_{3}^{2}$ & $\begin{array}{l}B W R \\
B W R\end{array}$ & $\begin{array}{l}3293 \\
3293\end{array}$ & $\begin{array}{l}4.02 \\
4.77\end{array}$ & $\begin{array}{l}\text { Yes } \\
\text { Yes }\end{array}$ & & & & & \\
\hline Peach bottom & TOTAL & & 6586 & 8.79 & & 6.4 & 5.3 & 11 & $4.1 E 6$ & $2.8 E-3$ \\
\hline pilgrim & 1 & BWR & 1998 & 2.65 & Yes & 0.093 & 52 & 52 & $4.4 E 6$ & 1. $2 E-2$. \\
\hline Point Beach & $\begin{array}{l}1 \\
2\end{array}$ & $\begin{array}{l}P W R \\
P W R\end{array}$ & $\begin{array}{l}1518 \\
1518\end{array}$ & $\begin{array}{l}3.59 \\
3.62\end{array}$ & - & & & & & \\
\hline Point Beach & OTAL & & 3036 & 7.31 & & 3.11 & 0.071 & 0.18 & $5.9 E 5$ & $3.0 E-4$ \\
\hline Proirie Island & $\frac{1}{2}$ & $\begin{array}{l}\text { PWR } \\
\text { PWR }\end{array}$ & $\begin{array}{l}1650 \\
1650\end{array}$ & $\begin{array}{l}3.71 \\
3.38\end{array}$ & - & & & & & \\
\hline Prairie jsland & TOTAL & & 3300 & 1.59 & & 6.27 & 0.32 & 0.59 & $2.1 \mathrm{EG}$ & $2.8 E-A$ \\
\hline Quad Cities & $\dot{z}$ & $\begin{array}{l}\text { BWR } \\
\text { aWR }\end{array}$ & $\begin{array}{l}2511 \\
7511\end{array}$ & $\begin{array}{l}3.52 \\
4.37\end{array}$ & $\begin{array}{l}y_{\text {eS }} \\
\text { Yes }\end{array}$ & & & & & \\
\hline Quad cities & TUTA. & & 5027 & 1.99 & & 3.7 & 1.3 & 4.0 & 6.775 & $5.9 E-3$ \\
\hline Rancho seco & 1 & DWR & 2172 & 5.98 & - & 0 & 0.064 & 0.064 & $1.5 E 6$ & $4,3 E-5$ \\
\hline H. B. Robenson & ] & PWR & 2200 & 4.23 & - & 0,45 & 0.024 & 0.47 & $6.4 F 5$ & $7.5 E-4$ \\
\hline St. Lucie & 1 & Piw & 2560 & 5.34 & - & $0.06 ?$ & 0.58 & 0.65 & $? .9 \mathrm{~Eb}$ & $2.3 E-3$ \\
\hline 5 alem & 1 & PWR & 3338 & 2.06 & - & 0.087 & 0.038 & 0.12 & 4.9E6 & $2.6 \mathrm{E}-5$ \\
\hline San Orofre & 1 & PWR & 1347 & 2.33 & - & 0,74 & 0.036 & 0.78 & $3.8 \mathrm{E} 6$ & $2.0 \mathrm{E}-4$ \\
\hline Surry & $\frac{?}{2}$ & $\begin{array}{l}\text { PWR } \\
\text { PWNR }\end{array}$ & $\begin{array}{l}2441 \\
7441\end{array}$ & $\begin{array}{l}5.02 \\
4.46\end{array}$ & - & & & & & \\
\hline Surry & TOTAL & & $488 ?$ & 9.18 & & 2.4 & 1.3 & 3.7 & $\therefore$. BEG & $? .15-3$ \\
\hline Three Mile Islatid & : & PWR & 7535 & 5.46 & - & 0.28 & 1.7 & $? .0$ & 3.955 & $1.1 \varepsilon-3$ \\
\hline Trojan & 1 & PwK & 3411 & 6.99 & - & 0.026 & 0.082 & 0.11 & 1.266 & $8.8 \mathrm{E}-5$ \\
\hline Turkey point & $\begin{array}{l}3 \\
4\end{array}$ & $\begin{array}{l}\text { PNR } \\
\text { PWR }\end{array}$ & $\begin{array}{l}2200 \\
2200\end{array}$ & $\begin{array}{l}4.47 \\
3.57\end{array}$ & - & & & & & \\
\hline Turkey $P_{i j i n t}$ & TO: AL & & 4400 & 8.14 & & 0.02 & 0.38 & $0.40^{\circ}$ & 7.166 & $2.0 E-4$ \\
\hline Vermont Yarike & 1 & BWhit & $: 593$ & 3.54 & Yes & 0.29 & 0.13 & 0.37 & $1.4 E 5$ & $2.6 E-4$ \\
\hline Yarkee Rowe & $\mathrm{i}$ & PAR & 600 & $\therefore, 03$ & - & $3 .: 5$ & 0.016 & 0,20 & $1.6 \mathrm{E} 6$ & 1. $3 E-4$ \\
\hline$z: 0 n$ & $\begin{array}{l}1 \\
?\end{array}$ & $\begin{array}{l}P W R \\
P_{N R R}\end{array}$ & $\begin{array}{l}3250 \\
3250\end{array}$ & $\begin{array}{l}3.07 \\
5.20\end{array}$ & - & & & & & \\
\hline zion & TOTQP, & & 6500 & -11.31 & & 13 & $-9 . \underline{5}$ & $m$ & $7.0 E \underline{6}$ & $3.7 E-3$ \\
\hline TOTAL FOR ALL SITES & & & & 246 & & 160 & 540 & 700 & 9.257 &.- \\
\hline Arithmet ic Medn & & & & 5.5 & & 3.5 & 12 & 16 & 2.0.05 & $1,1 E-2$ \\
\hline Geometric Mean & & & & 4.0 & & 0.29 & 0.30 & 1,3 & $1.1 F 6$ & $\therefore .2 E-3$ \\
\hline
\end{tabular}


Various site-dependent factors associated with the waterborne pathways are presented next. For lake and ocean sites, we used the average dilution of plant effluents for the year 1977 specified by Decker (1979). For river sites, the average annual river flow is tabulated. This flow was used in place of a dilution flow from the plant to account for dilutions of liquid releases at the locations of probable intake of drinking water and aquatic food catch. Any exceptions to this scheme have been footnoted. Next is shown the estimated 1977 population utilizing drinking water drawn from supplies containing diluted effluents from the site. These are shown with an estimated dilution factor where applicable. Fish and invertebrate catch data taken from the respective plant environmental statenent, when available (see Table 3 ), are listed next, along with estimated dilution factors for the lake and ocean sites. 


\section{RESULTS}

This report consists of a summary of values used for site-specific parameters at each site, as explained above, and the results of population dose commitment calculations. The population dose commitments are presented in two tables facing the page summarizing site-specific parameters for that site. These tables include both liquid and airborne pathway dose comitments for the several organs of reference for each age group investigated. They also include the dose to the whole population which includes all age groups. The airborne population dose commitments for each of 160 segments partitioning the region around the site(a) were divided by the population residing within that segment to derive an average individual dose for that segment. These doses are summarized as a histogram showing percent of the population receiving a given dose level for each site. The fractional population dose from the liquid pathway was not determined in this manner, because the NRC does not at present take into account the location of individuals exposed via this pathway, except those exposed through ingestion of drinking water.

Population dose commitments estimated for both the liquid pathways and airborne pathways varied widely over the 45 sites (60 reactors) studied. The total dose comitments (from both pathways) varied from a high of 220 to a low of 0.003 person-rem. The arithmetic mean for the dose from liquid pathways was 3 person-rem and the mean for the dose from airborne pathways was 12 person-rem (see Table 4).

Releases from Monticello, Rancho Seco and Dresden resulted in the smallest doses from liquid pathways (zero, zero, and near zero, respectively). This was because no liquid releases were reported for Montice 110 and Rancho Seco, and the receiving waters for the Dresden site are contaminated with Chicago sewage to such an extent as to severely limit use of this water for drinking or fishing. The largest liquid pathway doses to the total body calculated were 37 person-rem at Dconee and 35 person-rem at Hatch. These doses resulted primarily from the radioactive cesium (Cs-134 and Cs-137) released by these plants.

The lowest total airborne pathway dose to the total body was estimated for Palisades (0.0015 person-rem); while the highest were at Millstone Point (220 person-rem) and Dresden (180 person-rem). The major contributors to these doses were the noble gases: $\mathrm{Kr}-88$ and $\mathrm{Xe}-135$. The total population dose commitments from all sites for 1977 were estimated to be 160 person-rem via liquid pathways and 540 person-rem via the airborne pathways (Table 4). Compared to 1976, the "liquid dose" is higher (160 vs. 82); and also, the "air dose" is higher (540 vs. 390).

We should point out here, however, that the doses estimated in this study are extremely low compared to an average annual background dose of 0.1 rem,

(a) See Appendix for definition of segments. 
and they are well within all limits. Even the highest site average individua? dose commitment of 0.13 mrem (Table 4) from one site (Hatch) is well within any national limits.

Figure 1 shows graphically the wide range of the airborne population dose commitments for the reactor sites. The median, upper and lower quartiles and upper and lower octiles for the distribution of doses calculated for each of 160 segments are indicated for each site. Figure 2 is a histogram for all 45 sites taken together. We can see from this plot that about $25 \%$ of the tota? population at risk (92 million) would each receive a dose commitment of between 0.0003 and $0.001 \mathrm{mrem}$. We can see further that less than $0.5 \%$ receive a dose which is less than $1 \times 10^{-6}$ mrem. Although not discernible fram the plot, less than $0.01 \%$ received a dose of between 3 and 10 mrem. There were no average dose commitments greater than 10 mrem. However, no attempt was made in this study to estimate the maximum dose commitment received by any one individual from the radionuclides released at any of the sites.

It should be noted that we have been comparing dose comititments calculated in this study with annual background. This comparison is not quite exact, since these dose comitments are those total-body doses received from the year's (1977) effluent release, over 50 years of a person's lifetime. However, most of the dose comitment calculated here is delivered in the first year, so the comparison is reasonably valid. 


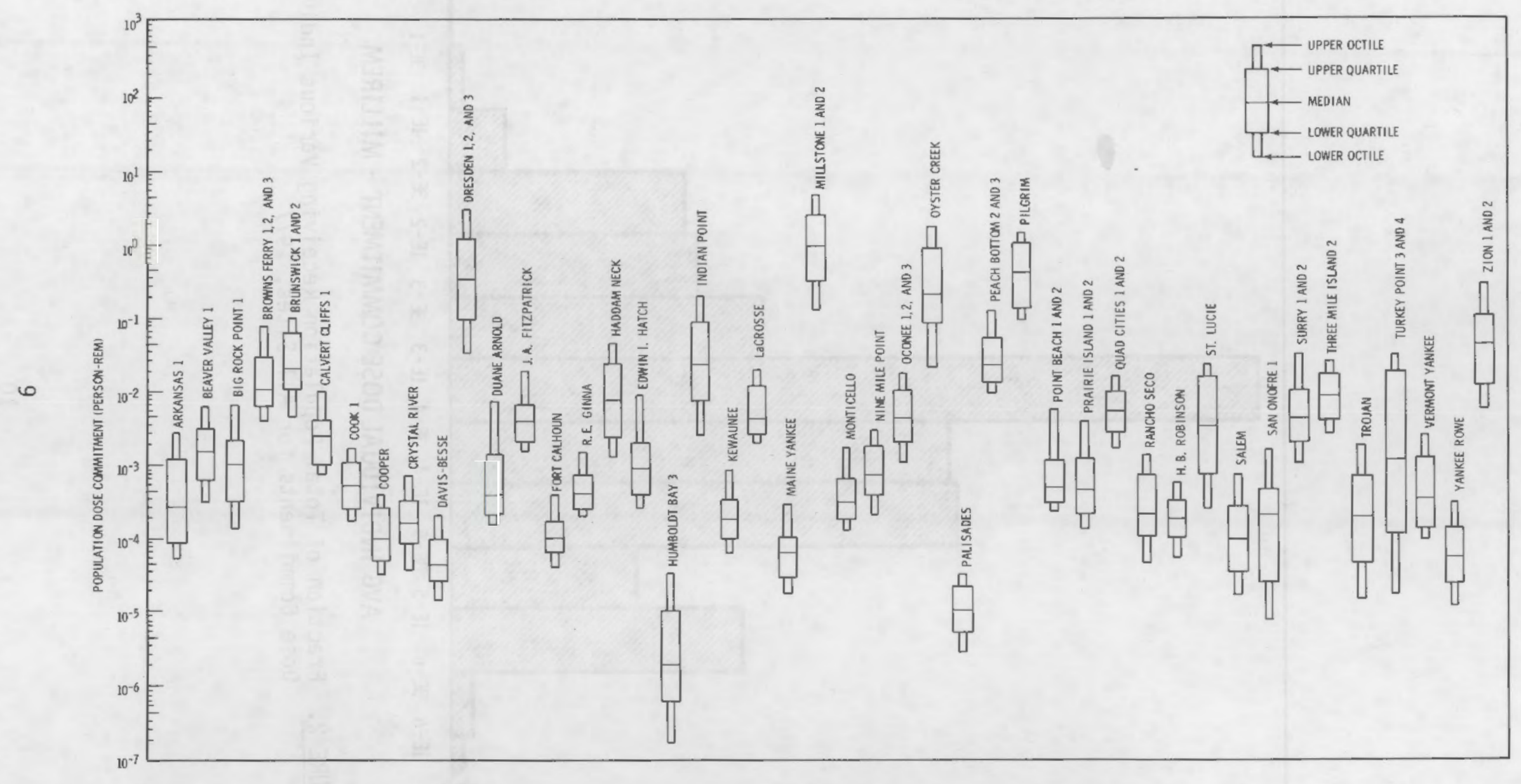

FIGURE 1. Airborne Population Dose Commitments for the Reactor Sites, 1977 


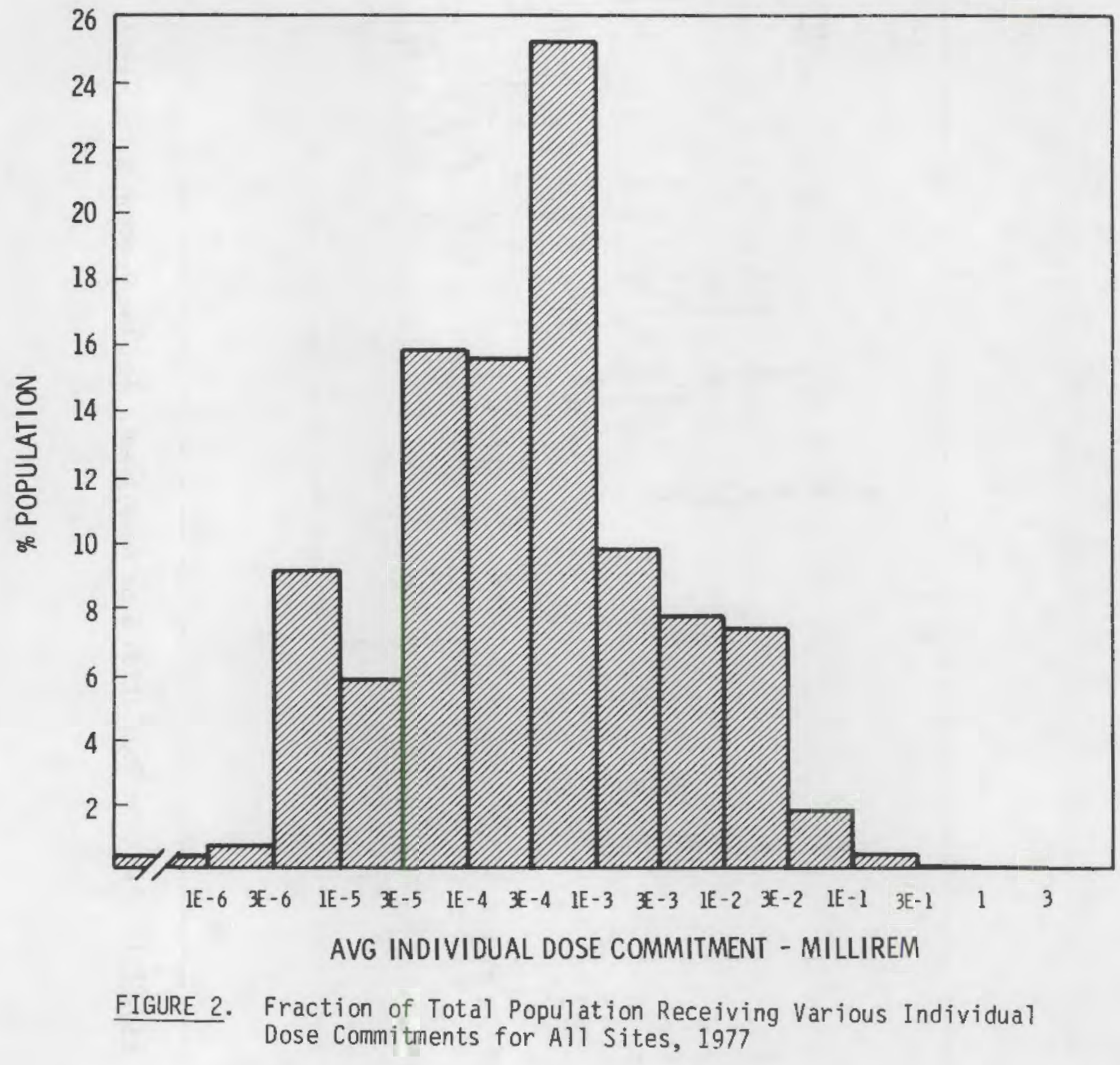


SITE SUMMARIES

1977 


Site $\frac{\text { ARKANSAS }}{\text { Location N } \frac{35.3075^{\circ}}{N}}$
Total Population Within 2-to-80-km Region

\begin{tabular}{l}
$\frac{\text { POPE COUNTY, ARKANSAS }}{\mathrm{W}} \frac{93.2308^{\circ}}{1.6 \mathrm{E} 5}$ \\
\hline
\end{tabular}

Major Metropolitan Centers Within Region

\begin{tabular}{l}
$\frac{\text { Center }}{\text { Russellville }}$ \\
\hline Conway \\
\hline
\end{tabular}

$\frac{\frac{\text { Population }}{13,000}}{17,000}$

$\begin{array}{r}\frac{}{2 \text { Location }} \\ 10 \mathrm{~km} \\ \hline 77 \mathrm{~km} \\ \hline\end{array}$

Average Annual State Production of Crops and Animal Products in $80-\mathrm{km}$ Radius Circle Veg $5.8 \mathrm{E} 6 \quad \mathrm{~kg} M i l \mathrm{k}+4.8 \mathrm{E} 7{ }^{\ell}$ Meat $7.2 \mathrm{E} 7$

Regional Productivity Factor

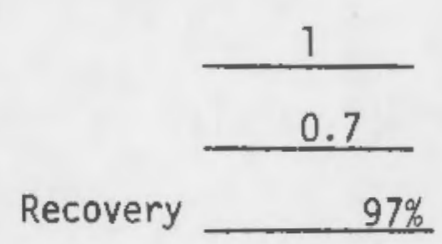

Animal Grazing Factor

Location of Meteorological Station Site Recovery

$97 \%$

Period of Record 1 JAN $75-31$ DEC 75

Average Arkansas___ River Flow at Site $36,000 \mathrm{ft}^{3} / \mathrm{s}$

Drinking Water

Exposed Population None_ Dilution Factor

Fish

Edible Harvest $1.4^{(\mathrm{a})} \mathrm{kg} / \mathrm{yr}$ Dilution Factor

(a) Average individual consumption rates as given in the FES (1973) were used in lieu of catch data. 


\begin{tabular}{|c|c|c|c|c|c|}
\hline & $\begin{array}{l}\text { POPUL } \\
\text { AVERAGE IN }\end{array}$ & $\begin{array}{l}\text { DOSE-COP } \\
\text { DUAL DOSE }\end{array}$ & $\begin{array}{l}\text { ENT ESTI } \\
\text { MITMENT }\end{array}$ & $\begin{array}{l}\text { AND } \\
\text { GRAM FOR }\end{array}$ & \\
\hline & Dose Commit & s (persor & ) from $L i$ & Pathways & \\
\hline & Total Body & GI-LLI & Thyroid & Bone & Liver \\
\hline $\begin{array}{l}\text { Infant } \\
\text { Child } \\
\text { Teen } \\
\text { Adult }\end{array}$ & $\begin{array}{l}0.0 \mathrm{E}+00 \\
6.5 \mathrm{E}-02 \\
1.2 \mathrm{E}-\mathrm{O1} \\
1.3 \mathrm{E}+00\end{array}$ & $\begin{array}{l}0.0 \mathrm{E}+00 \\
1.0 \mathrm{E}-02 \\
2.1 \mathrm{E}-02 \\
1.8 \mathrm{E}-01\end{array}$ & $\begin{array}{l}0.0 \mathrm{E}+00 \\
5.9 \mathrm{E}-03 \\
4.3 \mathrm{E}-03 \\
2.8 \mathrm{E}-02\end{array}$ & $\begin{array}{l}0.0 \mathrm{E}+00 \\
3.2 \mathrm{E}-01 \\
2.0 \mathrm{E}-01 \\
1.2 \mathrm{E}+00\end{array}$ & $\begin{array}{l}0.0 \mathrm{E}+00 \\
3.4 \mathrm{E}-01 \\
2.9 \mathrm{E}-01 \\
1.7 \mathrm{E}+00\end{array}$ \\
\hline TOTAL & $1.5 E+00$ & $2.1 E-01$ & $3.8 E-02$ & $1.7 E+00$ & $2.4 E+00$ \\
\hline
\end{tabular}

Dose Commitments (person-rem) from Airborne Pathways

\begin{tabular}{|c|c|c|c|c|c|c|}
\hline & Total Body & GI-LLI & Thyroid & Bone & Liver & Lung \\
\hline $\begin{array}{l}\text { Inf ant } \\
\text { Child } \\
\text { Teen } \\
\text { Adult }\end{array}$ & $\begin{array}{l}1.2 \mathrm{E}-03 \\
1.5 \mathrm{E}-02 \\
1.1 \mathrm{E}-02 \\
6.4 \mathrm{E}-02\end{array}$ & $\begin{array}{l}1.2 \mathrm{E}-03 \\
1.5 \mathrm{E}-02 \\
1.1 \mathrm{E}-02 \\
6.4 \mathrm{E}-02\end{array}$ & $\begin{array}{l}2.5 \mathrm{E}-03 \\
2.2 \mathrm{E}-02 \\
1.4 \mathrm{E}-02 \\
7.4 \mathrm{E}-02\end{array}$ & $\begin{array}{l}1.1 \mathrm{E}-03 \\
1.2 \mathrm{E}-02 \\
8.7 \mathrm{E}-03 \\
5.2 \mathrm{E}-02\end{array}$ & $\begin{array}{l}1.3 \mathrm{E}-03 \\
1.5 \mathrm{E}-02 \\
1.1 \mathrm{E}-02 \\
6.4 \mathrm{E}-02\end{array}$ & $\begin{array}{l}1.3 \mathrm{E}-03 \\
1.6 \mathrm{E}-02 \\
1.2 \mathrm{E}-02 \\
6.9 \mathrm{E}-\mathrm{D} 2\end{array}$ \\
\hline TOTAL & $9.0 \mathrm{E}-02$ & $9.0 \mathrm{E}-02$ & $1.1 E-01$ & $7.4 \mathrm{E}-02$ & $9.0 E-02$ & $9.8 \mathrm{E}-02$ \\
\hline
\end{tabular}

Production/consumption factors:

Produce: $<1$

Milk: 2.2

Meat: 5.4

FRACTIDN OF POPULATION RECEIVING AN INDICATED AVERAGE TOTAL-BODY DOSE COMMITMENT FROM AIRBORNE PATHWAYS

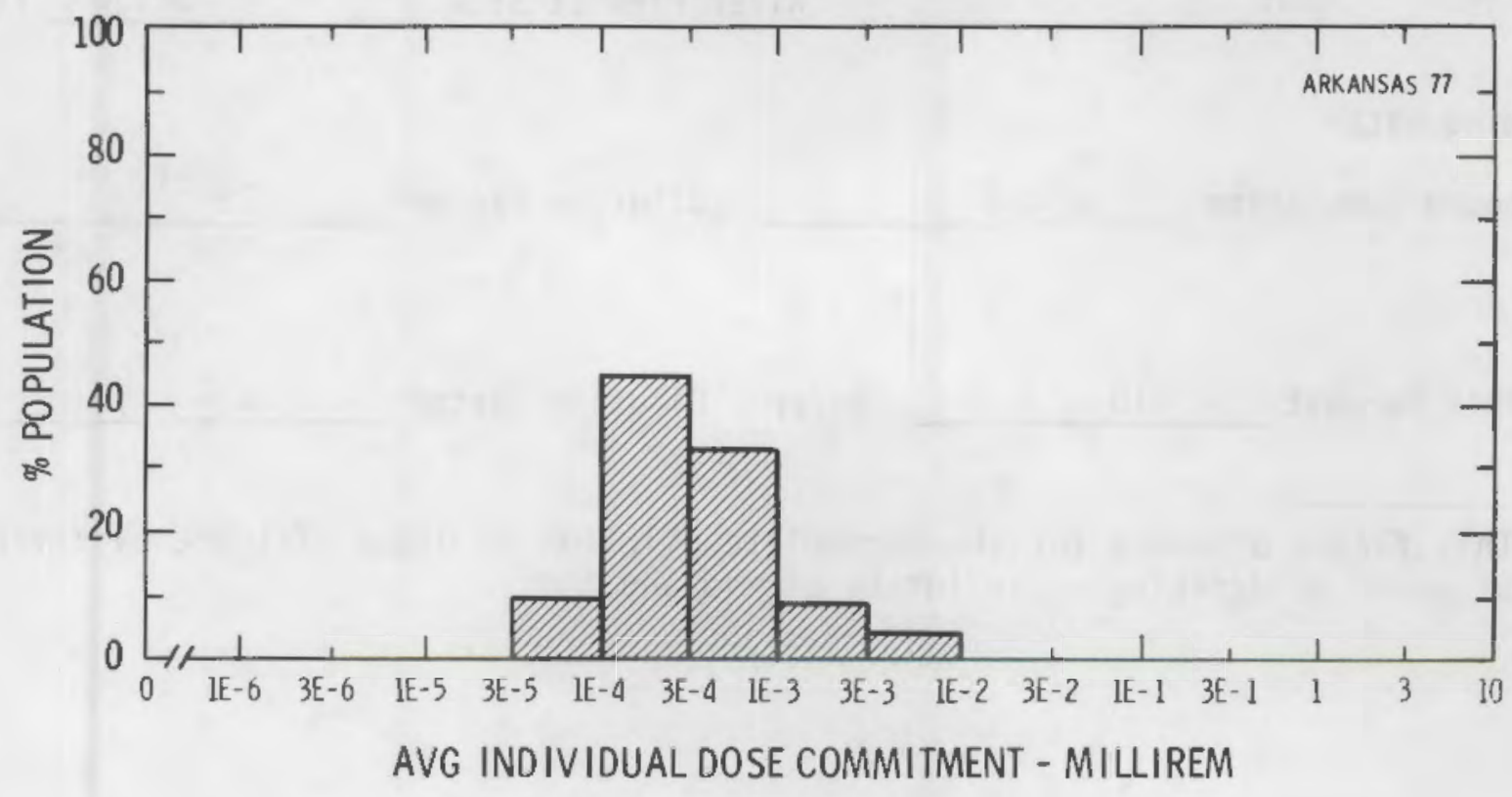


Site BEAVER VALLEY

Location $\mathrm{N} \quad 40.6214^{\circ}$

Total Population Within 2-to-80-km Region
SHIPPINGPORT, PENNSYI VANIA

W $80.4347^{\circ}$

Major Metropolitan Centers Within Region

\begin{tabular}{l}
\multicolumn{1}{c}{ Center } \\
Pittsburg SMSA \\
\hline Youngstown-Warren SMSA \\
\hline Steubenville \\
\hline Wheeling \\
\hline
\end{tabular}

Population

Location

Average Annual State Production of Crops and Animal Products in $80-\mathrm{km}$ Radius Circle

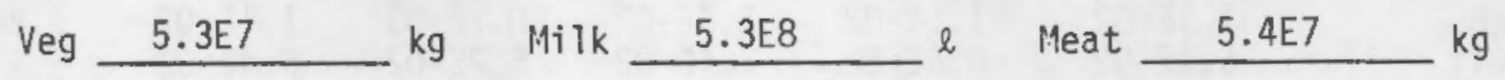

Regional Productivity Factor

\begin{tabular}{ll}
$32 \mathrm{~km}$ & $\mathrm{SE}$ \\
\hline $56 \mathrm{~km}$ & $\mathrm{NNW}$ \\
\hline $31 \mathrm{~km}$ & $\mathrm{SW}$ \\
\hline $69 \mathrm{~km}$ & $\mathrm{SSW}$ \\
\hline
\end{tabular}

Animal Grazing Factor

$\frac{1}{0.5}$

Location of Meteorological Station Site

Recovery $92 \%$

Period of Record

1 JAN 77 - 31 DEC 77

Average

Ohio

River Flow at Site

$30,000 \mathrm{ft}^{3} / \mathrm{s}$

Drinking Water

Exposed Population

6,400

Dilution Factor

${ }_{12}^{(a)}$

Fish

Edible Harvest 410 $\mathrm{kg} / \mathrm{yr}$ Dilution Factor 1

(a) This factor accounts for the incomplete dilution of plant effluent by river at point of drinking water intake at Midland. 


\begin{abstract}
POPULATION DOSE-COMMITMENT ESTIMATES ANO AVERAGE INDIVIOUAL OOSE-COMMITMENT HISTOGRAM FOR BEAVER VALLEY
\end{abstract}

\begin{tabular}{|c|c|c|c|c|c|}
\hline & Total Body & GI-LLI & Thyroid & Bone & Liver \\
\hline Inf ant & 3. $3 E-04$ & $2.8 \mathrm{E}-04$ & $3.1 E-04$ & $1.9 \mathrm{E}-04$ & $2.6 \mathrm{E}-04$ \\
\hline Child & $3.9 E-03$ & $3.6 \mathrm{E}-03$ & $3.1 \mathrm{E}-03$ & $2.9 \mathrm{E}-03$ & $2.8 \mathrm{E}-03$ \\
\hline Teen & $1.4 \mathrm{E}-03$ & $1.9 \mathrm{E}-03$ & $1.2 \mathrm{E}-03$ & $1.0 \mathrm{E}-03$ & $1.1 \mathrm{E}-03$ \\
\hline Adult & 02 & $1.7 \mathrm{E}$ & $9.7 E-03$ & $8.2 E-03$ & $9.0 E-03$ \\
\hline TOTAL & $1.7 \mathrm{E}-02$ & $2.3 E-02$ & $1.4 \mathrm{E}-02$ & 1. $2 E-02$ & $1.3 \mathrm{E}-02$ \\
\hline
\end{tabular}

Dose Commitments (person-rem) from Airborne Pathways

\begin{tabular}{|c|c|c|c|c|c|c|}
\hline & Total Body & GI-LLI & Thyroid & Bone & Liver & Lung \\
\hline $\begin{array}{l}\text { Infant } \\
\text { Child } \\
\text { Teen } \\
\text { Adult }\end{array}$ & $\begin{array}{l}6.7 \mathrm{E}-03 \\
7.9 \mathrm{E}-02 \\
4.9 \mathrm{E}-02 \\
2.5 \mathrm{E}-01\end{array}$ & $\begin{array}{l}6.7 \mathrm{E}-03 \\
7.9 \mathrm{E}-02 \\
4.9 \mathrm{E}-02 \\
2.5 \mathrm{E}-01\end{array}$ & $\begin{array}{l}7.0 \mathrm{E}-03 \\
8.0 \mathrm{E}-02 \\
4.9 \mathrm{E}-02 \\
2.5 \mathrm{E}-01\end{array}$ & $\begin{array}{l}5.9 \mathrm{E}-05 \\
7.2 \mathrm{E}-04 \\
5.1 \mathrm{E}-04 \\
3.0 \mathrm{E}-03\end{array}$ & $\begin{array}{l}6.7 \mathrm{E}-03 \\
7.9 \mathrm{E}-02 \\
4.9 \mathrm{E}-02 \\
2.5 \mathrm{E}-01\end{array}$ & $\begin{array}{l}6.7 \mathrm{E}-03 \\
7.9 \mathrm{E}-02 \\
4.9 \mathrm{E}-02 \\
2.5 \mathrm{E}-01\end{array}$ \\
\hline TOTAL & $3.8 E-01$ & $3.8 \mathrm{E}-01$ & $3.9 E-01$ & $4.3 E-03$ & $3.8 E-01$ & $3.9 \mathrm{E}-01$ \\
\hline
\end{tabular}

Production/consumption factors:

Produce: <1 Milk: $1.1 \quad$ Meat: <1

FRACTION OF POPULATION RECEIVING AN INDICATED AVERAGE

TOTAL-BODY DOSE COMMITMENT FROM AIRBORNE PATHWAYS

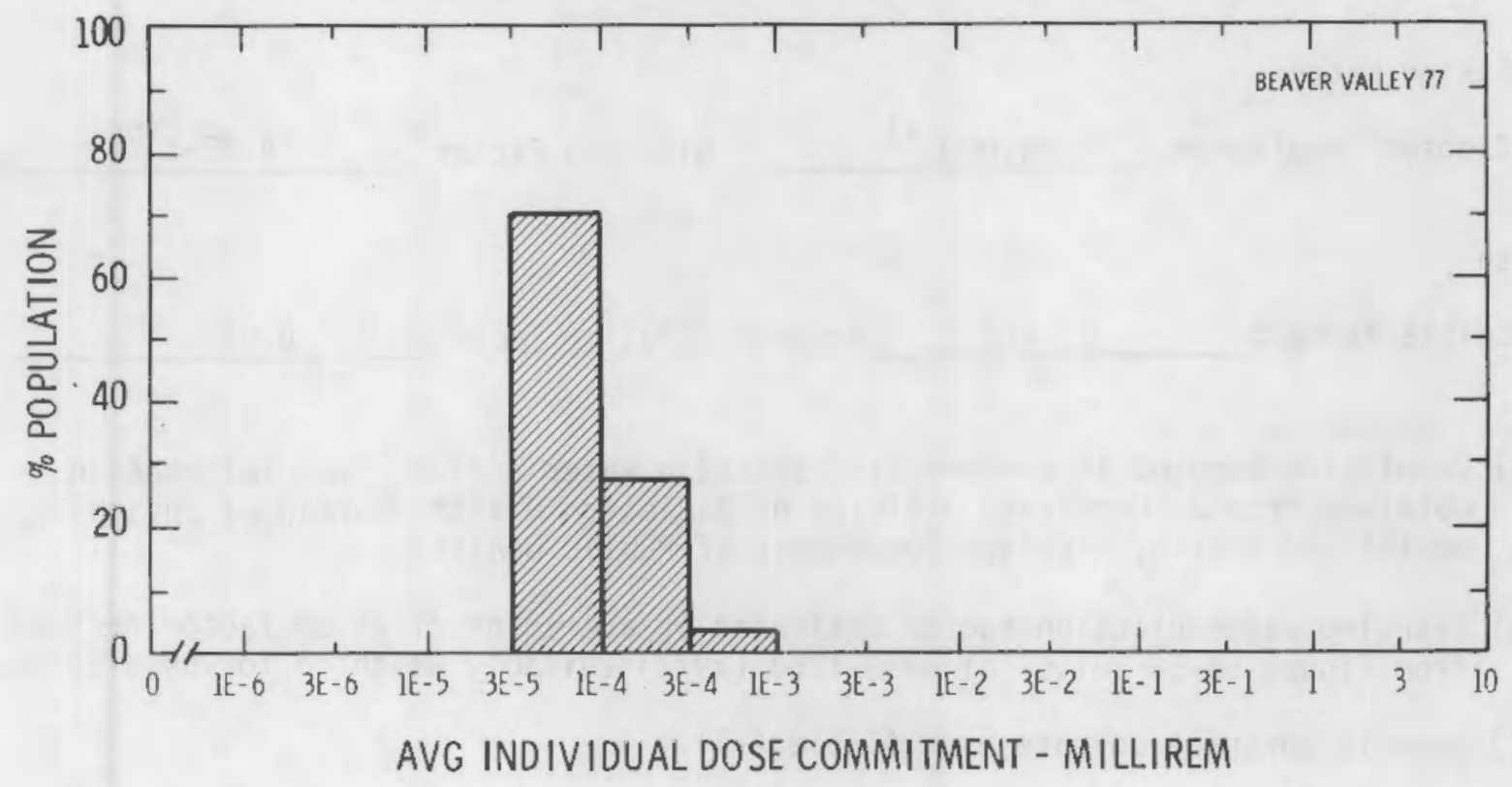


Site BIG ROCK POINT

Location $N$

N

$45.3589^{\circ}$

Total Population Within 2-to-80-km Region
CHARLEVOIX COUNTY. MICHIGAN

$85.1958^{\circ}$

$1.3 E 5$

Major Metropolitan Centers Within Region

Center

Traverse City
Population

18,000
Location

$70 \mathrm{~km}$

Average Annual State Production of Crops and Animal Products in 80-km Radius Circle

Veg $\frac{6.9 E 7}{\mathrm{~kg} \quad M i l k-2.9 \mathrm{E} 8{ }^{\ell} \text { Meat }{ }^{\ell} 4.5 \mathrm{E} 7} \mathrm{~kg}$

Regional Productivity Factor

0.5

Animal Grazing Factor

0.5

Location of Meteorological Station Site

Recovery

$85 \%$

Period of Record

9 FEB 61 - 8 FEB 63

Average Water Dilution Flow from Plant

$83 \mathrm{ft}^{3} / \mathrm{s}$

Drinking Water

Exposed Population

$24,000^{(a)}$

Dilution Factor

$4.9 E-5^{(b)}$

Fish

Edible Harvest.

(c) $\mathrm{kg} / \mathrm{yr}$ Dilution Factor

0.01

(a) Population exposed to contaminated drinking water derived from information obtained from J. Hennigan, Division of Radiation Health, Bureau of Environmental and Health, Michigan Department of Public Health.

(b) Drinking water dilution factor estimated by averaging dilution factor derived from Figure 6B-5, Vol. I of WASH-1258 (1973) suitably weighted for population.

(c) Generic consumption rate used (Table A-1). 


\section{POPULATION DOSE-COMMITMENT ESTIMATES AND}

AVERAGE INDIVIDUAL DOSE-COMMITMENT HISTOGRAM FOR BIG ROCK POINT

Dose Commitments (person-rem) from Liquid Pathways

\begin{tabular}{|c|c|c|c|c|c|}
\hline & Total Body & GI -LLI & Thyroid & Bone & Liver \\
\hline $\begin{array}{l}\text { Inf ant } \\
\text { Child } \\
\text { Teen } \\
\text { Adult }\end{array}$ & $\begin{array}{l}5.2 \mathrm{E}-07 \\
9.6 \mathrm{E}-02 \\
1.8 \mathrm{E}-01 \\
2.0 \mathrm{E}+00\end{array}$ & $\begin{array}{l}2.1 \mathrm{E}-07 \\
6.2 \mathrm{E}-03 \\
1.3 \mathrm{E}-02 \\
1.1 \mathrm{E}-01\end{array}$ & $\begin{array}{l}1.2 \mathrm{E}-07 \\
2.5 \mathrm{E}-05 \\
1.9 \mathrm{E}-05 \\
1.4 \mathrm{E}-04\end{array}$ & $\begin{array}{l}3.0 \mathrm{E}-06 \\
5.4 \mathrm{E}-01 \\
3.2 \mathrm{E}-01 \\
1.9 \mathrm{E}+00\end{array}$ & $\begin{array}{l}3.7 E-06 \\
5.8 E-01 \\
4.8 E-01 \\
2.9 E+00\end{array}$ \\
\hline TOTAL & $2.3 E+00$ & $1.3 \mathrm{E}-01$ & $1.8 \mathrm{E}-04$ & 2. $7 \mathrm{E}+00$ & $3.9 E+00$ \\
\hline
\end{tabular}

Dose Commitments (person-rem) from Airborne Pathways

\begin{tabular}{|c|c|c|c|c|c|c|}
\hline & Total Body & GI-LLI & Thyroid & Bone & Liver & Lung \\
\hline Inf ant & 4. $0 E-03$ & $4.0 E-03$ & 4. $1 \mathrm{E}-03$ & 4.0E-03 & $4.0 E-03$ & $4.1 E-03$ \\
\hline d & 4. $4 \mathrm{E}-02$ & $4.4 \mathrm{E}-02$ & $4.6 \mathrm{E}-02$ & $4.4 \mathrm{E}-02$ & $4.4 \mathrm{E}-02$ & $4.6 \mathrm{E}-02$ \\
\hline S & $3.2 \bar{E}-02$ & 3.2E-D2 & $3.3 \mathrm{E}-02$ & $3.2 \mathrm{E}-02$ & $3.2 \mathrm{E}-02$ & $3.4 \mathrm{E}-02$ \\
\hline $1 \mathrm{t}$ & & 2. & 2 & 01 & -01 & 2. $0 \mathrm{E}-01$ \\
\hline JTAL & $2.8 \mathrm{E}-01$ & $2.8 \mathrm{E}-01$ & $2.8 \mathrm{E}-01$ & $2.8 \mathrm{E}-01$ & $2.8 E-01$ & 2.9E-01 \\
\hline
\end{tabular}

Production/consumption factors:

Produce: 1.4 Milk: 8.5

Meat: 2.2

FRACTION OF POPULATION RECEIVING AN INDICATED AVERAGE TOTAL-BODY DOSE COMMITMENT FROM AIRBORNE PATHWAYS

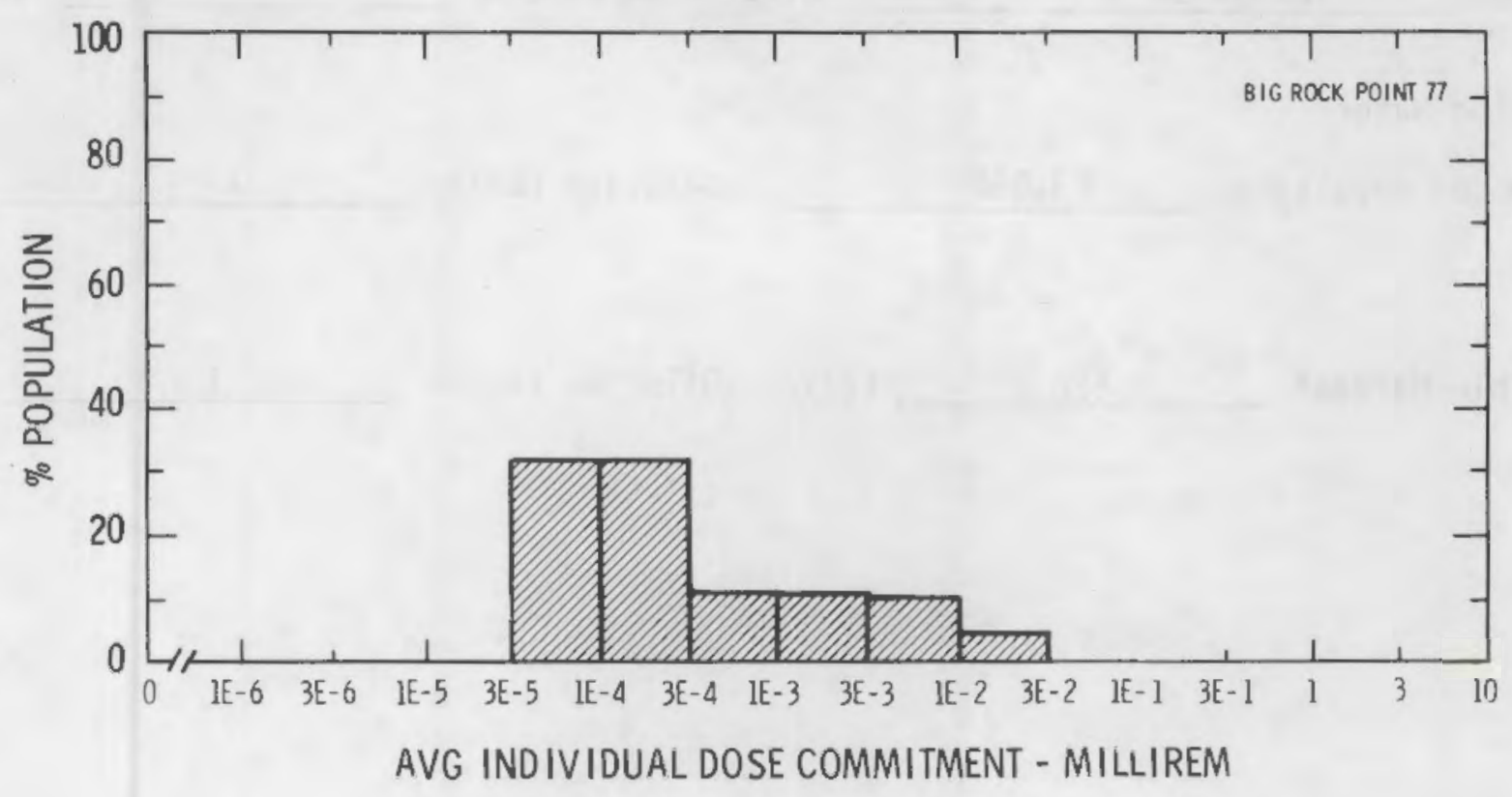


Site BROWNS FERRY

Location $\mathrm{N} 34.7036^{\circ}$

Total Population Within 2-to-80-km Region
DECATUR, ALABAMA

W $87.1211^{\circ}$

6.7 E5

Major Metropolitan Centers Within Region

\begin{tabular}{l} 
Decatur $^{\text {Center }}$ \\
\hline Huntsville \\
\hline
\end{tabular}

$\frac{\frac{\text { Population }}{40,000}}{150,000}$

\begin{tabular}{ll}
$\frac{2}{2}$ Location \\
$16 \mathrm{~km}$ & $\mathrm{SE}$ \\
\hline $48 \mathrm{~km}$ & $\mathrm{E}$ \\
\hline
\end{tabular}

Average Annual State Production of Crops and Animal Products in $80-\mathrm{km}$ Radius C'ircle Veg $1.7 \mathrm{E} 7$ $\mathrm{kg} \quad \mathrm{Milk}$ $5.7 \mathrm{E} 7$ \& Meat 8.6E7 $\mathrm{kg}$

Regional Productivity Factor

Animal Grazing Factor $\frac{1}{0.7}$

Location of Meteorological Station Site Recovery $94 \%$

Period of Record 1 JAN $74-31$ DEC 75

Average Tennessee River Flow at Site $45,000 \mathrm{ft}^{3} / \mathrm{s}$

Drinking Water

Exposed Population 23,000

Dilution Factor 1

Fish

Edible Harvest 1.6E6 $\mathrm{kg} / \mathrm{yr}$ Dilution Factor 1 


\section{POPULATION DOSE-COMMITMENT ESTIMATES AND \\ AVERAGE INDIVIDUAL DOSE-COMMITMENT HISTOGRAM FOR \\ BROWNS FERRY $1,2 \& 3$}

Dose Commitments (person-rem) from Liquid Pathways

Infant

Child

Teen

Adult

TOTAL

\begin{tabular}{|c|c|c|c|c|}
\hline Total Body & GI-LLI & Thyroid & Bone & Liver \\
\hline $4.1 E-05$ & $\overline{3.1 E-05}$ & $\overline{1.5 E-03}$ & $1.3 \mathrm{E}-04$ & $9.6 \mathrm{E}-05$ \\
\hline $3.4 \mathrm{E}-02$ & $4.1 E-02$ & $2.0 E-02$ & 1. $2 \mathrm{E}-01$ & $1.4 \mathrm{E}-01$ \\
\hline $4.8 \mathrm{E}-02$ & $8.6 E-02$ & 1. $0 \mathrm{E}-02$ & $7.1 \mathrm{E}-02$ & $1.2 E-01$ \\
\hline & & $6.7 \mathrm{E}-02$ & $4.2 E-01$ & $7.0 \mathrm{E}-01$ \\
\hline $5.5 \mathrm{E}-01$ & $8.8 E-01$ & $9.8 \mathrm{E}-02$ & $6.1 E-01$ & $9.5 \mathrm{E}-01$ \\
\hline
\end{tabular}

Dose Commitments (person-rem) from Airborne Pathways

\begin{tabular}{|c|c|c|c|c|c|c|}
\hline & Total Body & GI-LLI & Thyroid & Bone & Liver & Lung \\
\hline $\begin{array}{l}\text { Inf ant } \\
\text { Child } \\
\text { Teen } \\
\text { Adult }\end{array}$ & $\begin{array}{l}3.8 \mathrm{E}-02 \\
4.3 \mathrm{E}-01 \\
3.1 \mathrm{E}-01 \\
1.9 \mathrm{E}+00\end{array}$ & $\begin{array}{l}3.8 \mathrm{E}-02 \\
4.2 \mathrm{E}-01 \\
3.1 \mathrm{E}-01 \\
1.9 \mathrm{E}+00\end{array}$ & $\begin{array}{l}5.0 E-02 \\
5.0 E-01 \\
3.4 E-01 \\
2.0 E+00\end{array}$ & $\begin{array}{l}3.8 \mathrm{E}-02 \\
4.3 \mathrm{E}-01 \\
3.1 \mathrm{E}-01 \\
1.9 \mathrm{E}+00\end{array}$ & $\begin{array}{l}3.9 E-02 \\
4.3 E-01 \\
3.1 E-01 \\
1.9 E+00\end{array}$ & $\begin{array}{l}4.2 E-02 \\
4.8 E-01 \\
3.8 E-01 \\
2.1 E+00\end{array}$ \\
\hline TOTAL & $2.7 E+00$ & $2.7 \mathrm{E}+00$ & $2.9 \mathrm{E}+00$ & $2.7 \mathrm{E}+00$ & $2.7 \mathrm{E}+00$ & $3.0 \mathrm{E}+00$ \\
\hline
\end{tabular}

Production/consumption factors:
Produce: $<1$
Milk: $<1$
Meat: 1.6

FRACTION OF POPULATION RECEIVING AN INDICATED AVERAGE

TOTAL-BODY DOSE COMMITMENT FROM AIRBORNE PATHWAYS

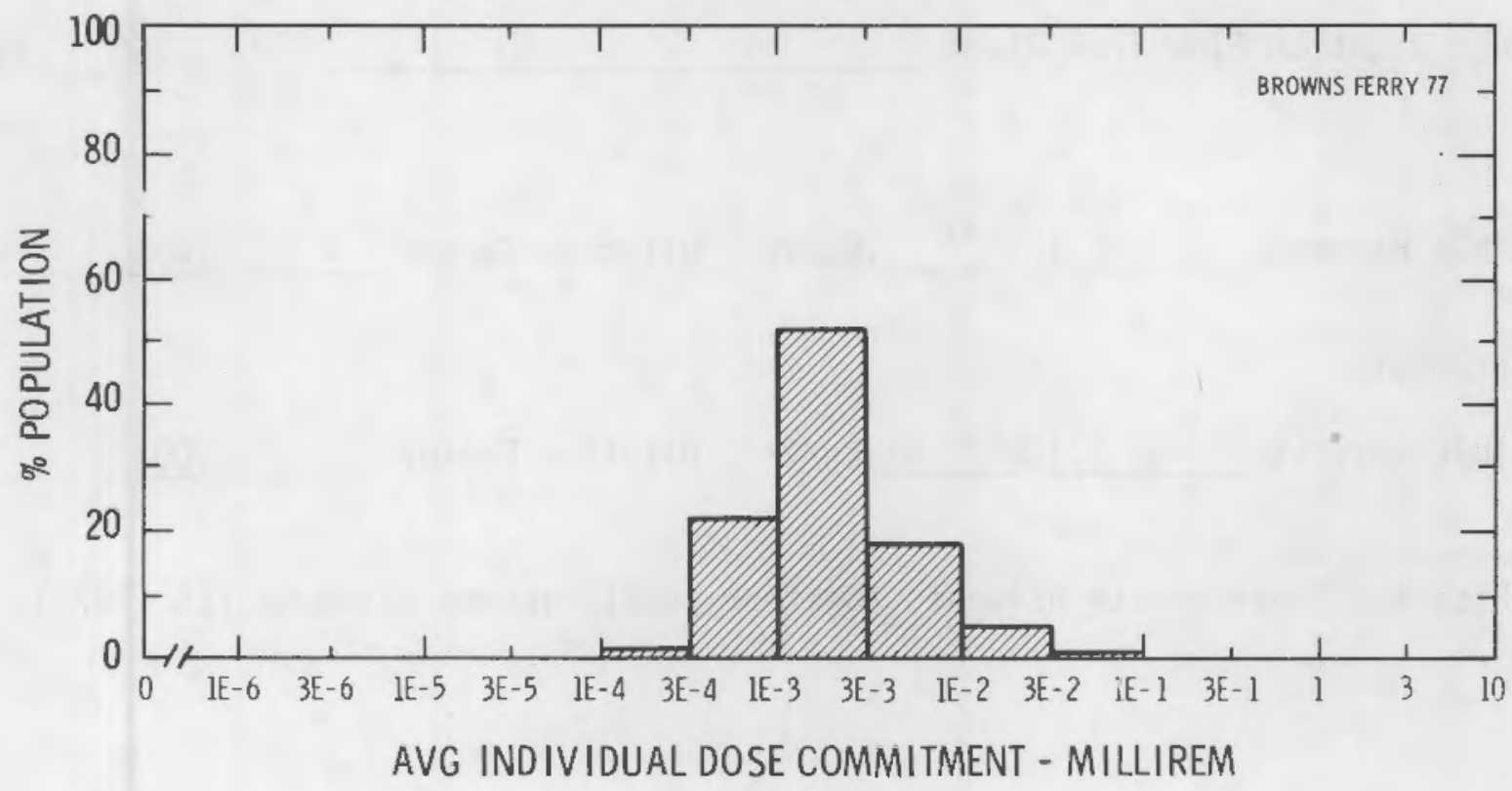


Site BRUNSWICK

Location $\mathrm{N}$ $33.9586^{\circ}$

Total Population Within 2-to-80-km Region
BRUNSWICK COUNTY, NORTH CAROLINA

W $78.0208^{\circ}$

Major Metropolitan Centers Within Region

Center

Wilmington
Population

51,000

\section{Location} N

Average Annual State Production of Crops and Animal Products in 80-km Radius Circle Veg 2.6E7 $\mathrm{kg}$ Milk $1.0 \mathrm{E} 8$ \& Meat $5.8 E 7$ $\mathrm{kg}$

Regional Productivity Factor

0.3

Animal Grazing Factor

0.7

Location of Meteorological Station Site Recovery $93 \%$

Period of Record 1 JAN $75-31$ DEC 75

Average Dilution Flow from Plant 250 $f t^{3} / s$

Fish

Edible Harvest $2.1 E 5^{(a)}$ $\mathrm{kg} / \mathrm{yr}$ Dilution Factor 0.001 Invertebrates Edible Harvest $1.1 \mathrm{E} 5^{(\mathrm{a})}$ $\mathrm{kg} / \mathrm{yr}$ Dilution Factor 0.002

(a) Fish and invertebrate harvests together total harvest given in FES (1973). 


\begin{tabular}{|c|c|c|c|c|c|c|}
\hline & \multicolumn{5}{|c|}{$\begin{array}{l}\text { POPULATION DOSE-COMMITMENT ESTIMATES AND } \\
\text { AVERAGE INOIVIDUAL DOSE-COMMITMENT HISTOGRAM FOR } \\
\text { BRUNSWICK } 1 \& 2\end{array}$} & \multirow{2}{*}{ Liver } \\
\hline & \multicolumn{6}{|l|}{$=$} \\
\hline & Total Body & GI-LLI & & Thyroid & Bone & \\
\hline $\begin{array}{l}\text { Infant } \\
\text { Child } \\
\text { Teen } \\
\text { Adult }\end{array}$ & $\begin{array}{l}0.0 \mathrm{E}+00 \\
1.1 \mathrm{E}-02 \\
7.6 \mathrm{E}-03 \\
4.7 \mathrm{E}-02\end{array}$ & $\begin{array}{l}0.0 \mathrm{E} \\
1.0 \mathrm{E} \\
2.1 \mathrm{E} \\
1.8 \mathrm{E}\end{array}$ & $\begin{array}{l}0.1 \\
7.2 \\
5.2 \\
3.2\end{array}$ & $\begin{array}{l}+00 \\
-03 \\
-03 \\
-02\end{array}$ & $\begin{array}{l}0.0 \mathrm{E}+00 \\
9.2 \mathrm{E}-03 \\
5.9 \mathrm{E}-03 \\
3.8 \mathrm{E}-02\end{array}$ & $\begin{array}{l}0.0 \mathrm{E}+00 \\
1.9 \mathrm{E}-02 \\
1.7 \mathrm{E}-02 \\
1.0 \mathrm{E}-01\end{array}$ \\
\hline TOTAL & $6.6 \mathrm{E}-02$ & $2.2 \mathrm{E}$ & 4.7 & -02 & $5.3 E-02$ & $1.4 E-01$ \\
\hline & Dose Conmitn & nts (pe & rem) $f$ & Airborne & e Pathways & \\
\hline & Total Body & GI-LLI & Thyroid & Bone & Liver & Lung \\
\hline $\begin{array}{l}\text { Inf ant } \\
\text { Child } \\
\text { Teen } \\
\text { Adult }\end{array}$ & $\begin{array}{l}9.0 \mathrm{E}-02 \\
1.0 \mathrm{E}+00 \\
7.4 \mathrm{E}-01 \\
4.5 \mathrm{E}+00\end{array}$ & $\begin{array}{l}9.0 \mathrm{E}-02 \\
1.0 \mathrm{E}+00 \\
7.3 \mathrm{E}-01 \\
4.4 \mathrm{E}+00\end{array}$ & $\begin{array}{l}1.8 \mathrm{E}-01 \\
1.6 \mathrm{E}+00 \\
9.5 \mathrm{E}-01 \\
5.2 \mathrm{E}+00\end{array}$ & $\begin{array}{l}9.3 \mathrm{E}-02 \\
1.1 \mathrm{E}+00 \\
7.9 \mathrm{E}-01 \\
4.7 \mathrm{E}+00\end{array}$ & $\begin{array}{l}9.0 \mathrm{E}-02 \\
1.0 \mathrm{E}+00 \\
7.3 \mathrm{E}-01 \\
4.4 \mathrm{E}+00\end{array}$ & $\begin{array}{l}9.2 \mathrm{E}-02 \\
1.0 \mathrm{E}+00 \\
7.8 \mathrm{E}-01 \\
4.6 \mathrm{E}+00\end{array}$ \\
\hline TOTAL & $6.3 E+00$ & $6.2 \mathrm{E}+00$ & $7.9 E+00$ & $6.7 E+00$ & $6.2 E+00$ & $6.5 E+00$ \\
\hline
\end{tabular}

Production/consumption factors:

Produce: $<1 \quad$ Milk: $1.3 \quad$ Meat: 1.2

FRACTION OF POPULATION RECEIVING AN INDICATED AVERAGE

TOTAL-BODY DOSE COMMITMENT FROM AIRBORNE PATHWAYS

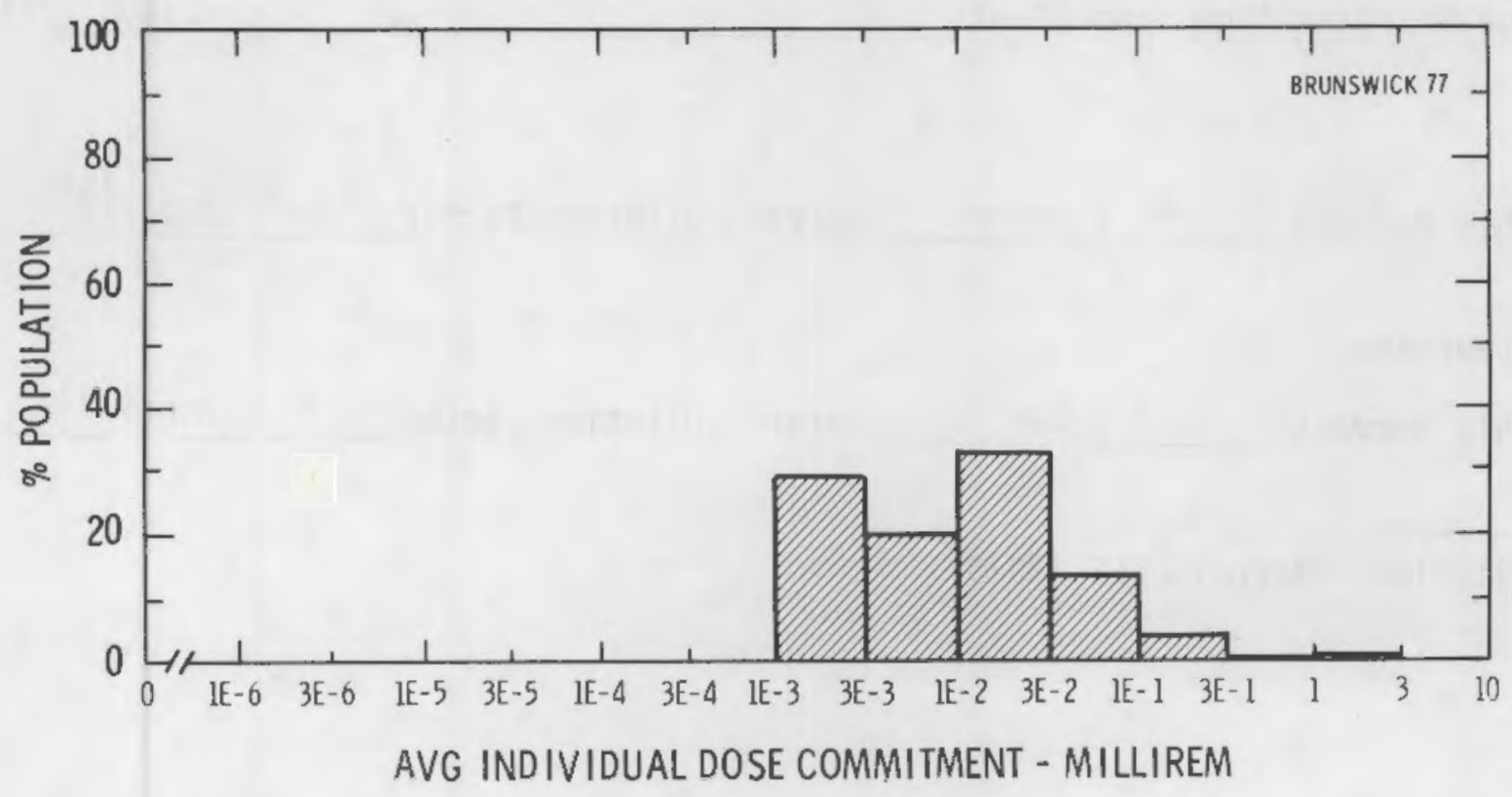


Site CALVERT CLIFFS

LUSBY, MARYLAND

Location $\mathrm{N}$

$38.4272^{\circ}$

Total Population Within 2-to-80-km Region

W $\quad 76.4261^{\circ}$

$2.4 \mathrm{E} 6$

Major Metropolitan Centers Within Region

\begin{tabular}{l}
$\frac{\text { Center }}{\text { Washington, OC SMSA }(3 / 4}$ \\
Cambridge \\
\hline Annapolis \\
\hline
\end{tabular}

$\begin{array}{r}\frac{\text { Population }}{2,300,000} \\ \hline 12,000 \\ \hline 31,000 \\ \hline\end{array}$

\begin{tabular}{|c|c|}
\hline $75 \mathrm{~km}$ & NW \\
\hline $32 \mathrm{~km}$ & ENE \\
\hline 6] $\mathrm{km}$ & $\mathrm{N}$ \\
\hline
\end{tabular}

Average Annual State Production of Crops and Animal Products in $80-\mathrm{km}$ Radius Circle Veg kg Milk $5.0 \mathrm{E} 8$ \& Meat $\mathrm{kg}$

Regional Productivity Factor

Site $\quad \frac{0.6}{\frac{0.6}{96 \%}}$

Location of Meteorological Station Site 1 JAN $75-31$ DEC 75

Period of Record

Average Dilution Flow from Plant $2,400 \quad \mathrm{ft}^{3} / \mathrm{s}$

Fish

Edible Harvest 1. 0 E7 kg/yr Dilution Factor $0.062^{(a)}$

Invertebrates
Edible Harvest
$7.4 \mathrm{E} 6$
$\mathrm{kg} / \mathrm{yr}$ Dilution Factor
$0.062^{(a)}$

(a) Dilutions given in FES (1973). 


\begin{abstract}
POPULATION DOSE-COMMITMENT ESTIMATES AND AVERAGE INDIVIDUAL DOSE-COMMITMENT HISTOGRAM FOR CALVERT CLIFFS
\end{abstract}

Dose Commitments (person-rem) from Liquid Pathways

Infant

Child

Teen

Adult

TOTAL

\begin{tabular}{l} 
Total Body \\
\hline $0.0 \mathrm{E}+00$ \\
$1.2 \mathrm{E}-01$ \\
$1.2 \mathrm{E}-01$ \\
$9.9 \mathrm{E}-01$ \\
$1.2 \mathrm{E}+00$
\end{tabular}

$\frac{G I-L L I}{0.0 E+00}$
$1.6 \mathrm{E}+00$
$3.4 \mathrm{E}+00$
$3.0 \mathrm{E}+01$
$3.5 \mathrm{E}+01$

$\frac{\text { Thyroid }}{0.0 \mathrm{E}+00}$

3. $3 \mathrm{E}-01$

2. $4 \mathrm{E}-01$

$1.6 E+00$

$2.1 \mathrm{E}+00$ $\frac{\text { Bone }}{0.0 E+00}$

2.2E-01

1. $3 \mathrm{E}-01$

8. $0 \mathrm{E}-01$

$1.2 \mathrm{E}+00$ $\frac{\text { Liver }}{0.0 \mathrm{E}+00}$

2.7E-01

2. $3 \mathrm{E}-01$

1. $4 \mathrm{E}+00$

$1.9 \mathrm{E}+00$

Dose Commitments (person-rem) from Airborne Pathways

\begin{tabular}{|c|c|c|c|c|c|}
\hline Total Body & GI-LLI & Thyroid & Bone & Liver & Lung \\
\hline $\begin{array}{l}1.1 \mathrm{E}-02 \\
1.2 \mathrm{E}-01 \\
8.3 \mathrm{E}-02 \\
4.9 \mathrm{E}-01\end{array}$ & $\begin{array}{l}1.0 \mathrm{E}-02 \\
1.2 \mathrm{E}-01 \\
8.3 \mathrm{E}-02 \\
4.9 \mathrm{E}-01\end{array}$ & $\begin{array}{l}2.2 \mathrm{E}-01 \\
1.2 \mathrm{E}+00 \\
5.1 \mathrm{E}-01 \\
1.8 \mathrm{E}+00\end{array}$ & $\begin{array}{l}9.7 \mathrm{E}-03 \\
1.0 \mathrm{E}-01 \\
7.5 \mathrm{E}-02 \\
4.5 \mathrm{E}-01\end{array}$ & $\begin{array}{l}1.1 \mathrm{E}-02 \\
1.2 \mathrm{E}-01 \\
8.4 \mathrm{E}-02 \\
4.9 \mathrm{E}-01\end{array}$ & $\begin{array}{l}1.2 \mathrm{E}-02 \\
1.3 \mathrm{E}-01 \\
1.0 \mathrm{E}-01 \\
5.6 \mathrm{E}-01\end{array}$ \\
\hline 7.0E-01 & 7.0E-01 & $3.8 \mathrm{E}+00$ & $6.4 \mathrm{E}-01$ & 7.1E-01 & 8.0E-01 \\
\hline
\end{tabular}

Production/consumption factors:
Produce: $<1$
Milk: $<1$
Meat: $<1$

FRACTION OF POPULATION RECEIVING AN INDICATED AVERAGE

TOTAL-BOOY DOSE COMMITMENT FROM AIRBORNE PATHWAYS

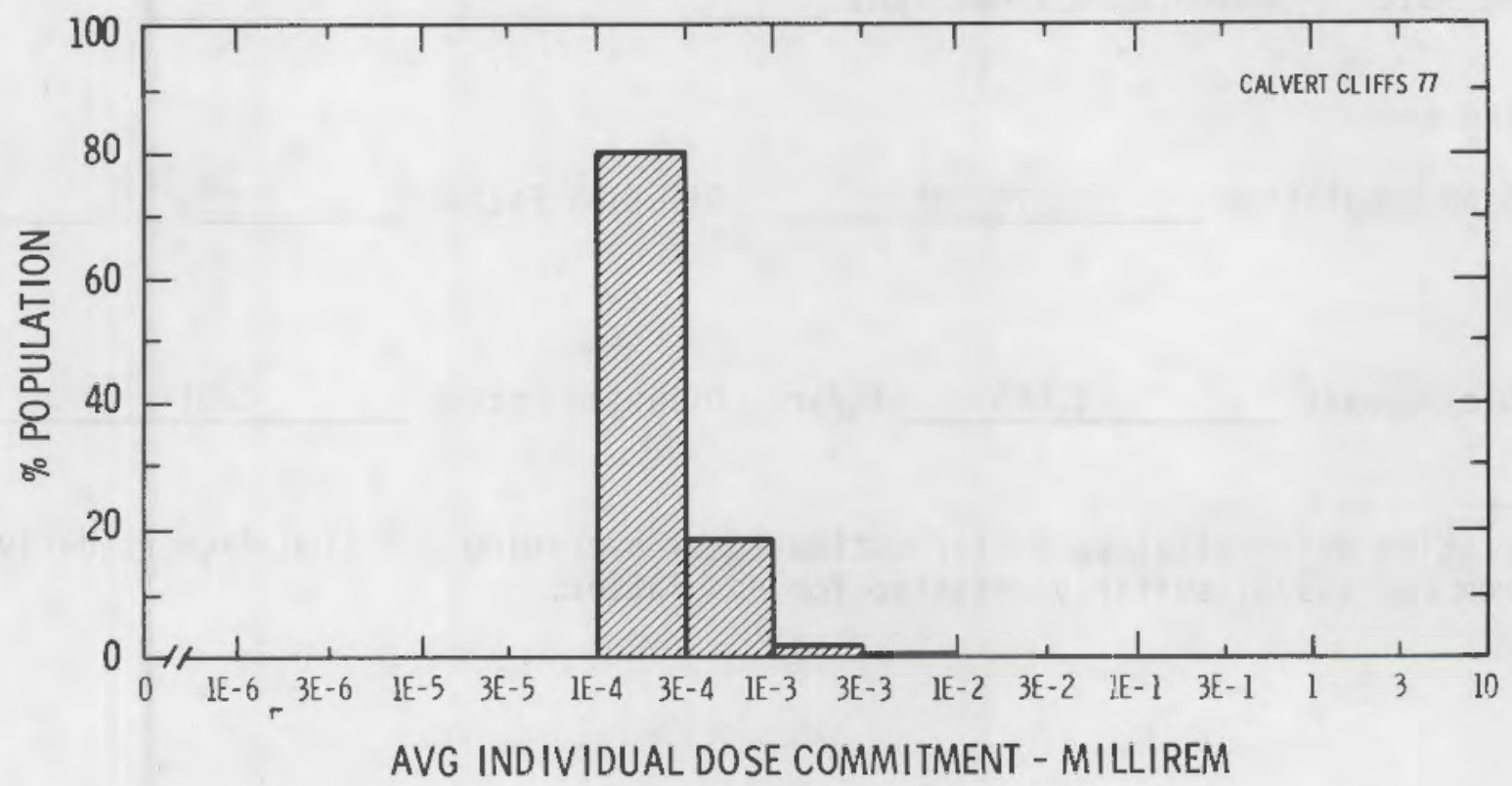


Site COOK

Location $\mathrm{N} \quad 41.9742^{\circ}$

Total Population Within 2-to-80-km Region
BENTON HARBOR, MICHIGAN

W $86.5667^{\circ}$

Major Metropolitan Centers Within Region

Center

Gary SMSA

South Bend SMSA

Benton Harbor
Population 650,000

290.000

17,000

Location

\begin{tabular}{|c|c|c|}
\hline 650,000 & $80 \mathrm{~km}$ & SW \\
\hline 290.000 & $42 \mathrm{~km}$ & SE \\
\hline 17,000 & $18 \mathrm{~km}$ & $\mathrm{NE}$ \\
\hline
\end{tabular}

Average Annual State Production of Crops and Animal Products in $80-\mathrm{km}$ Radius Circle Veg $1.1 \mathrm{E} 8 \mathrm{~kg} \quad \mathrm{Milk}$ 2.3E8 \& Meat $1.9 E 8$ $\mathrm{kg}$

Regional Productivity Factor

Animal Grazing Factor 0.6

Location of Meteorologic gical Station

Site Recovery 0.5

Period of Record 1 MAY 75 - 31 APR 76

Average Water Dilution Flow from Plant $78 \mathrm{ft}^{3} / \mathrm{s}$

Drinking Water

$$
\text { Exposed Population }
$$
270,000

Dilution Factor $0.025^{(a)}$

Fish

Edible Harvest 1.5E6 $\mathrm{kg} / \mathrm{yr}$ Dilution Factor 0.01

(a) Drinking water dilution factor estimated by averaging dilution factors derived from FES (1973) suitably weighted for population. 


\begin{abstract}
POPULATION DOSE-COMMITMENT ESTIMATES AND AVERAGE INDIVIDUAL DOSE-COMMITMENT HISTOGRAM FOR COOK
\end{abstract}

Infant

Child

Teen

Adult

TOTAL

Inf ant
Child
Teen
Adult
TOTAL

Production/consumption factors:

Produce: $<1$

\begin{tabular}{|c|c|}
\hline GI-LLI & Thyroid \\
\hline $\begin{array}{l}2.3 E-02 \\
4.6 E-01 \\
5.1 E-01 \\
4.4 E+00\end{array}$ & $\begin{array}{l}8.8 \mathrm{E}-02 \\
7.1 \mathrm{E}-01 \\
2.4 \mathrm{E}-01 \\
1.8 \mathrm{E}+00\end{array}$ \\
\hline $5.4 E+00$ & $2.9 \mathrm{E}+00$ \\
\hline
\end{tabular}

$\frac{\text { Bone }}{5.5 E-02}$

$\frac{\text { Liver }}{1 . \mathrm{DE}-01}$

$4.2 E+00$

$2.4 \mathrm{E}+00$

$1.4 \mathrm{E}+01$

$5.5 \mathrm{E}+00$

$1.8 \mathrm{E}+00$

2. $0 \mathrm{E}+01$

$5.4 \mathrm{E}+00$

$2.1 \mathrm{E}+01$

$4.3 \mathrm{E}+00$

2. $6 \mathrm{E}+01$

2. $3 \mathrm{E}+01$

Dose Commitments (person-rem) from Airborne Pathways

\begin{tabular}{|c|c|c|}
\hline Total Body & GI-LLI & Thyroid \\
\hline $\begin{array}{l}8.3 \mathrm{E}-04 \\
9.8 \mathrm{E}-03 \\
7.5 \mathrm{E}-03 \\
4.4 \mathrm{E}-02\end{array}$ & $\begin{array}{l}7.1 \mathrm{E}-04 \\
8.0 \mathrm{E}-03 \\
5.8 \mathrm{E}-03 \\
3.5 \mathrm{E}-02\end{array}$ & $\begin{array}{l}3.0 E-02 \\
2.1 E-01 \\
8.2 E-02 \\
2.9 E-01\end{array}$ \\
\hline $6.3 E-02$ & 5.0E-02 & $6.1 \mathrm{E}-01$ \\
\hline
\end{tabular}

$\frac{\text { Bone }}{1.5 E-03}$
$1.6 \mathrm{E}-02$
$8.3 \mathrm{E}-03$
$4.3 \mathrm{E}-02$
$6.9 \mathrm{E}-02$

$\frac{\text { Liver }}{1.9 \mathrm{E}-03}$

$1.8 \mathrm{E}-02$

$1.0 E-02$

$4.8 \mathrm{E}-02$

$\frac{\text { Lung }}{9.7 \mathrm{E}-04}$

$1.1 \mathrm{E}-02$

9.1E-03

4. $5 E-02$

TOTAL

Production/consump
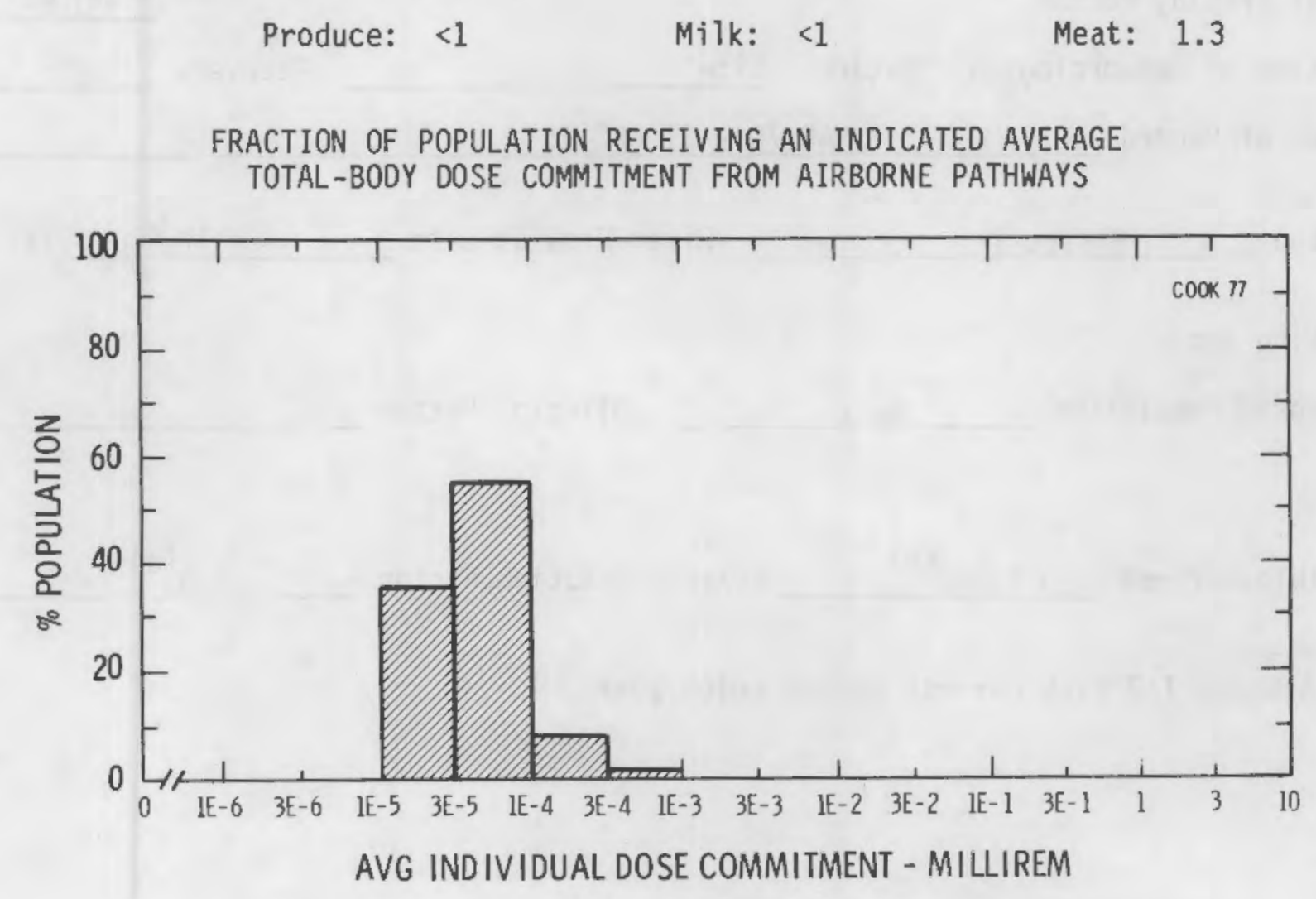
Site

COOPER STATION

Location $\mathrm{N} \quad 40.3614^{\circ}$

Total Population Within 2-to-80-km Region
NEMAHA COUNTY, NEBRASKA

W $95.6378^{\circ}$

Major Metropolitan Centers Within Region

Center

Shenandoah
Population

6,000
Location

$58 \mathrm{~km}$ NNE

Average Annual State Production of Crops and Animal Products in $80-\mathrm{km}$ Radius Circle

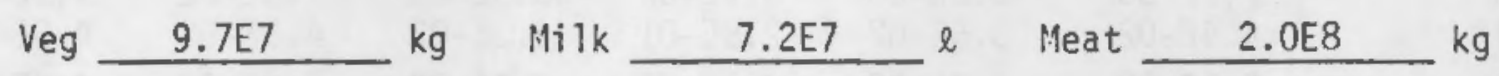

Regional Productivity Factor 1

Animal Grazing Factor 0.6

Location of Meteorological Station Site Recovery $89 \%$

Period of Record 1 MAR $70-31$ DEC 75

Average Missouri

River Flow at Site 31,000 $\mathrm{ft}^{3} / \mathrm{s}$

Drinking Water

Exposed Poputation None Dilution Factor

Fish

Edible Harvest $5.03^{(a)}$ $\mathrm{kg} / \mathrm{yr}$ Dilution Factor $0.5^{(a)}$

(a) Assumes 1/2 fish harvest caught below plant. 


\section{POPULATION DOSE-CDMMITMENT ESTIMATES AND AVERAGE INDIVIDUAL DOSE-COMMITMENT HISTOGRAM FOR COOPER STATION}

Infant
Child
Teen
Adult
TOTAL

Dose Commitments (person-rem) from Liquid Pathways

\begin{tabular}{|c|c|c|c|c|}
\hline Total Body & GI-LLI & Thyroid & Bone & Liver \\
\hline $\begin{array}{l}0.0 \mathrm{E}+00 \\
1.6 \mathrm{E}-05 \\
2.7 \mathrm{E}-05 \\
2.8 \mathrm{E}-04\end{array}$ & $\begin{array}{l}0.0 \mathrm{E}+00 \\
1.0 \mathrm{E}-05 \\
2.2 \mathrm{E}-05 \\
2.0 \mathrm{E}-04\end{array}$ & $\begin{array}{l}0.0 E+00 \\
3.7 E-06 \\
2.6 E-06 \\
1.7 E-05\end{array}$ & $\begin{array}{l}0.0 \mathrm{E}+00 \\
7.6 \mathrm{E}-05 \\
4.6 \mathrm{E}-05 \\
2.7 \mathrm{E}-04\end{array}$ & $\begin{array}{l}0.0 E+00 \\
7.9 E-05 \\
6.8 E-05 \\
4.0 E-04\end{array}$ \\
\hline 3. $2 E-04$ & $2.3 \mathrm{E}-04$ & $2.4 \mathrm{E}-05$ & $3.9 E-04$ & $5.5 E-04$ \\
\hline
\end{tabular}

Dose Commitments (person-rem) from Airborne Pathways

\begin{tabular}{|c|c|c|c|c|c|c|}
\hline & Total Body & GI-LLI & Thyroid & Bone & Liver & Lung \\
\hline $\begin{array}{l}\text { Inf ant } \\
\text { Child } \\
\text { Teen } \\
\text { Adult }\end{array}$ & $\begin{array}{l}2.1 \mathrm{E}-04 \\
3.0 \mathrm{E}-03 \\
2.0 \mathrm{E}-03 \\
1.2 \mathrm{E}-02\end{array}$ & $\begin{array}{l}2.1 \mathrm{E}-04 \\
2.9 \mathrm{E}-03 \\
2.0 \mathrm{E}-03 \\
1.2 \mathrm{E}-02\end{array}$ & $\begin{array}{l}2.0 \mathrm{E}-03 \\
2.0 \mathrm{E}-02 \\
8.7 \mathrm{E}-03 \\
3.5 \mathrm{E}-02\end{array}$ & $\begin{array}{l}1.8 E-04 \\
2.5 E-03 \\
1.6 E-03 \\
9.3 E-03\end{array}$ & $\begin{array}{l}2.2 E-04 \\
3.0 E-03 \\
2.0 E-03 \\
1.1 E-02\end{array}$ & $\begin{array}{l}2.2 E-04 \\
3.0 E-03 \\
2.1 E-03 \\
1.2 E-02\end{array}$ \\
\hline TTAL & 1. $7 \mathrm{E}-02$ & $1.7 \mathrm{E}-02$ & $6.7 \mathrm{E}-02$ & $1.4 \mathrm{E}-02$ & $1.7 \mathrm{E}-02$ & 1. $7 \mathrm{E}-02$ \\
\hline
\end{tabular}

Production/consumption factors:

Produce: 2.8 Milk: $3.1 \quad$ Meat: 14

FRACTION OF POPULATION RECEIVING AN INDICATED AVERAGE TOTAL-BODY DOSE COMMITMENT FROM AIRBORNE PATHWAYS

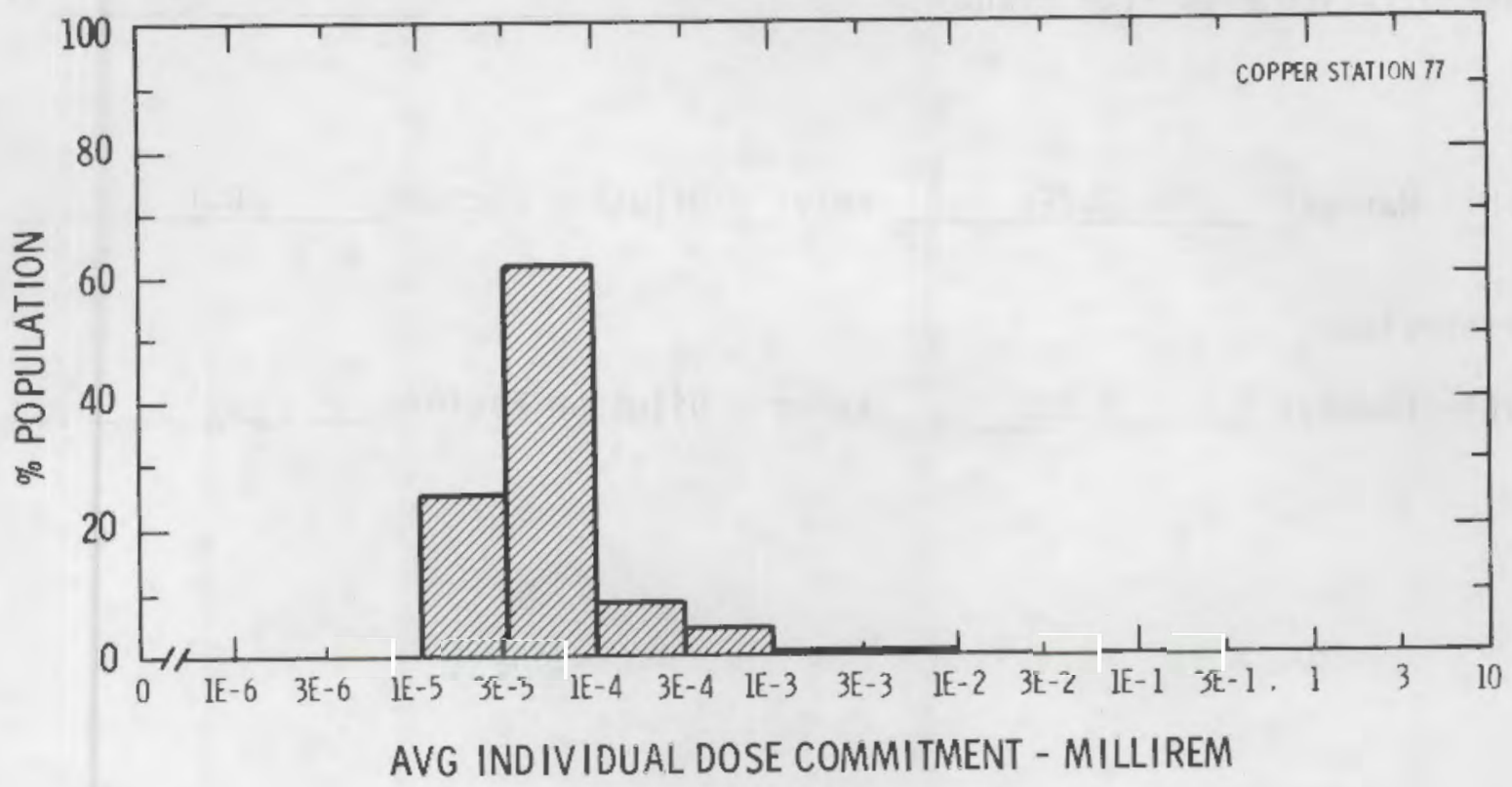


Site CRYSTAL RIVER

Location N $28.9567^{\circ}$

Total Population Within 2-to-80-km Region
CRYSTAL RIVER, FLORIDA

$82.6980^{\circ}$

Major Metropolitan Centers Within Region

Center

Ocala
Population

29,000
Location

$63 \mathrm{~km}$

Average Annual State Production of Crops and Anima? Products in $80-\mathrm{km}$ Radius Circle

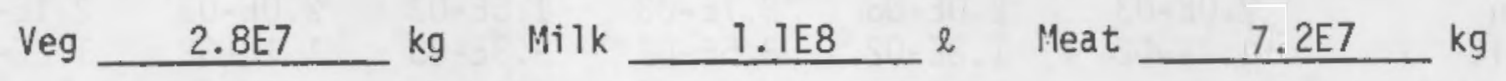

Regional Productivity Factor 0.5

Animal Grazing Factor

Location of Meteorological Station Site

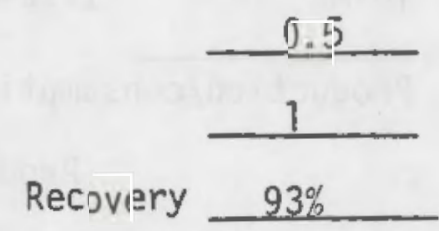

Period of Record 1 JAN $75-31$ DEC 75

Average Dilution Flow from Plant 244 $\mathrm{ft}^{3} / \mathrm{s}$

Fish

Edible Harvest 3.2E5 $\mathrm{kg} / \mathrm{yr}$ Dilution Factor 0.1 Invertebrates Edible Harvest $1.8 \mathrm{E} 5$ $\mathrm{kg} / \mathrm{yr}$ Dilution Factor 0.1 


\section{POPULATION OOSE-COMMITMENT ESTIMATES ANO AVERAGE INDIVIDUAL OOSE-COMMITMENT HISTOGRAM FOR CRYSTAL RIVER}

Dose Commitments (person-rem) from Liquid Pathways

\begin{tabular}{|c|c|c|c|c|c|}
\hline & Total Body & GI-LLI & Thyroid & Bone & Liver \\
\hline $\begin{array}{l}\text { Inf ant } \\
\text { Child } \\
\text { Teen } \\
\text { Aduit }\end{array}$ & $\begin{array}{l}0.0 E+00 \\
8.9 E-04 \\
7.4 E-04 \\
5.5 E-03\end{array}$ & $\begin{array}{l}0.0 \mathrm{E}+00 \\
1.3 \mathrm{E}-03 \\
2.2 \mathrm{E}-03 \\
1.9 \mathrm{E}-02\end{array}$ & $\begin{array}{l}0.0 E+00 \\
7.3 E-03 \\
5.2 E-03 \\
3.5 E-02\end{array}$ & $\begin{array}{l}0.0 E+00 \\
5.0 E-04 \\
3.2 E-04 \\
2.0 E-03\end{array}$ & $\begin{array}{l}0.0 E+00 \\
1.1 E-03 \\
9.3 E-04 \\
6.4 E-03\end{array}$ \\
\hline TOTAL & $7.2 \mathrm{E}-03$ & 2. $3 \mathrm{E}-02$ & 4.7E-02 & 2. $8 \mathrm{E}-03$ & $8.4 E-03$ \\
\hline
\end{tabular}

Dose Commitments (person-rem) from Airborne Pathways

\begin{tabular}{|c|c|c|c|c|c|c|}
\hline & Total Body & GI-LLI & Thyroid & Bone & Liver & Lung \\
\hline $\begin{array}{l}\text { Infant } \\
\text { Child } \\
\text { Teen } \\
\text { Adult }\end{array}$ & $\begin{array}{l}2.0 E-04 \\
2.6 E-03 \\
1.7 E-03 \\
1.0 E-02\end{array}$ & $\begin{array}{l}2.0 \mathrm{E}-04 \\
2.6 \mathrm{E}-03 \\
1.7 \mathrm{E}-03 \\
1.0 \mathrm{E}-02\end{array}$ & $\begin{array}{l}5.3 E-04 \\
4.5 E-03 \\
2.4 E-03 \\
1.2 E-02\end{array}$ & $\begin{array}{l}1.3 \mathrm{E}-04 \\
1.4 \mathrm{E}-03 \\
1.0 \mathrm{E}-03 \\
6.3 \mathrm{E}-03\end{array}$ & $\begin{array}{l}2.0 \mathrm{E}-04 \\
2.6 \mathrm{E}-03 \\
1.7 \mathrm{E}-03 \\
1.0 \mathrm{E}-02\end{array}$ & $\begin{array}{l}2.1 \mathrm{E}-04 \\
2.7 \mathrm{E}-03 \\
1.9 \mathrm{E}-03 \\
1.0 \mathrm{E}-02\end{array}$ \\
\hline TOTAL & $1.4 \mathrm{E}-02$ & $1.5 \mathrm{E}-02$ & 2.0E-02 & 9.0E-03 & 1.5E-02 & $1.5 \mathrm{E}-02$ \\
\hline
\end{tabular}

Production/consumption factors:

Produce: $<1 \quad$ Milk: $1.9 \quad$ Meat: 2.0

FRACTION OF POPULATION RECEIVING AN INOICATED AVERAGE TOTAL-BOOY DOSE COMMITMENT FROM AIRBORNE PATHWAYS

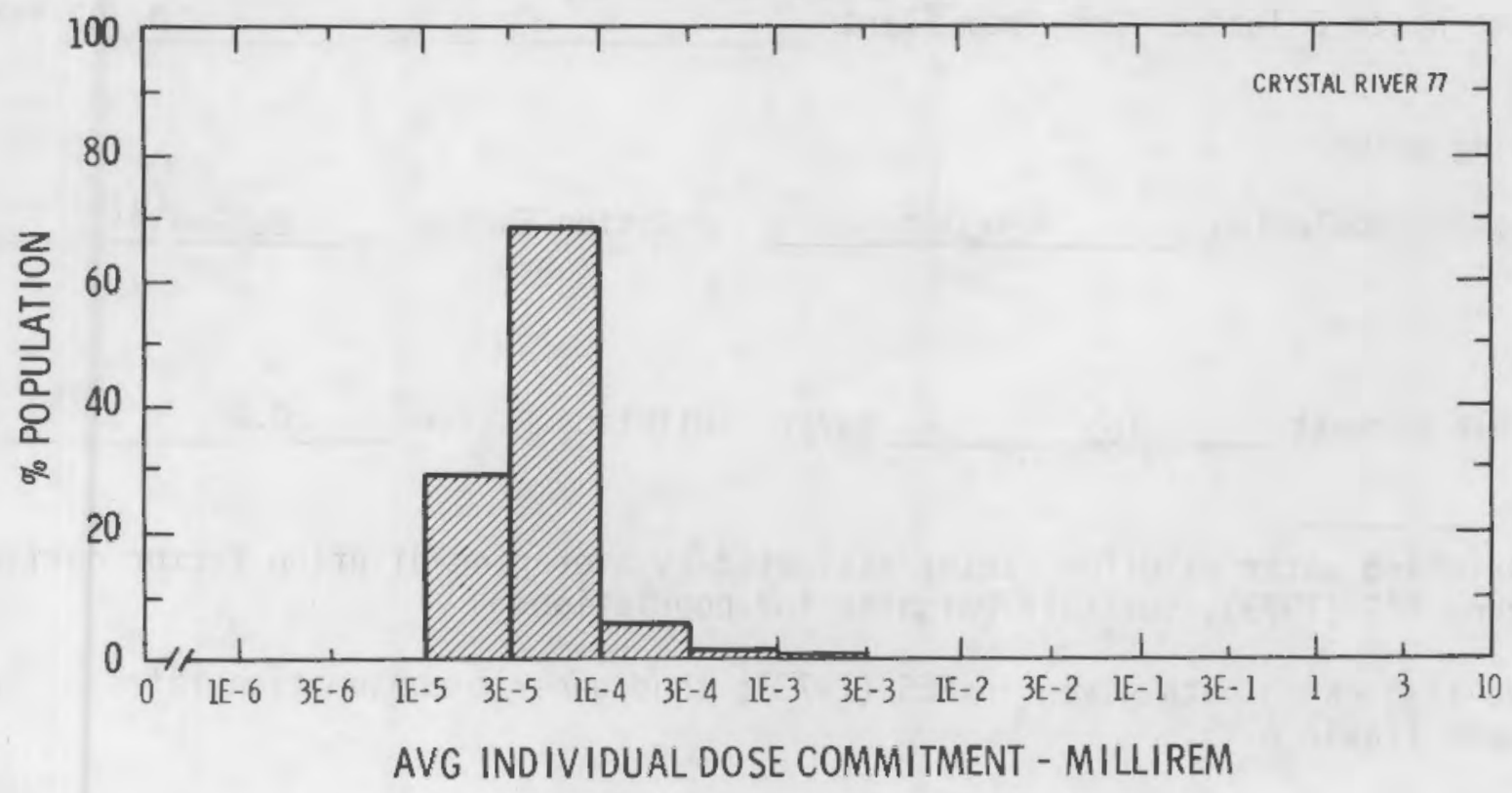


Site DAVIS-BESSE

Location $N$ $41.5947^{\circ}$

Total Population Within 2-to-80-km Region
PORT CLINTON, OHIO

W $83.0864^{\circ}$

1.8E6

Major Metropolitan Centers Within Region

\begin{tabular}{l} 
Toledo \\
Sandusky \\
\hline Findlay \\
\hline
\end{tabular}

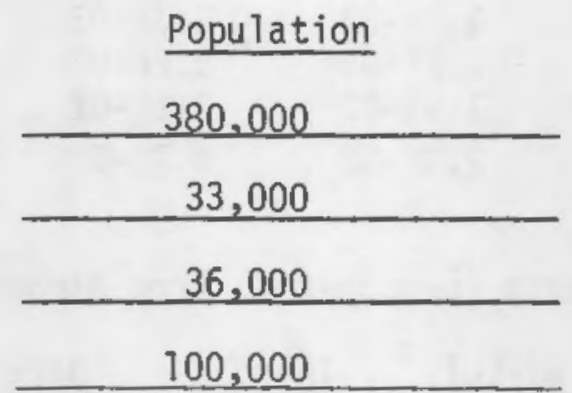

\begin{tabular}{ll}
\multicolumn{2}{l}{ Location } \\
$37 \mathrm{~km}$ & WNW \\
\hline $35 \mathrm{~km}$ & ESE \\
\hline $77 \mathrm{~km}$ & SW \\
\hline $80 \mathrm{~km}$ & $\mathrm{~N}$ \\
\hline
\end{tabular}

Average Annual State Production of Crops and Animal Products in $80-\mathrm{km}$ Radius Circle

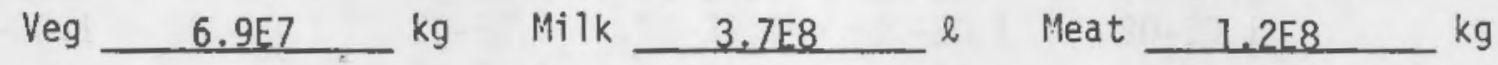

Regional Productivity Factor

Animal Grazing Factor

0.5

Location of Meteorological Station Site

Recovery $99 \%$

Period of Record

4. AUG $74-3$ AUG 76

Average Water Dilution Flow from Plant

$0.707 \mathrm{ft}^{3} / \mathrm{s}$

Drinking Water

Exposed Population 450,000

Dilution Factor

2. $0 E-4^{(a)}$

Fish

Edible Harvest (b) $\mathrm{kg} / \mathrm{yr}$ Dilution Factor 0.01

(a) Drinking water dilution factor estimated by averaging dilution factor derived from FES (1973), suitably weighted for population.

(b) No fish catch data given in FES (1973); thus generic consumption rates used (Table A-1). 


\section{POPULATION DOSE-COMMITMENT ESTIMATES AND AVERAGE INOIVIDUAL DOSE-COMMITMENT HISTOGRAM FOR DAVIS-BESSE}

Oose Commitments (person-rem) from Liquid Pathways

\begin{tabular}{|c|c|c|c|c|c|}
\hline & Total Body & GI-LLI & Thyroid & Bone & Liver \\
\hline Inf ant & 1. 3E-03 & $8.6 \bar{E}-04$ & $\overline{1.3 \mathrm{E}-03}$ & $2.1 E-03$ & $8.7 E-04$ \\
\hline Child & 1. $0 E+00$ & 7.1E-01 & $9.6 \mathrm{E}-02$ & $3.9 E+00$ & $2.4 \mathrm{E}+00$ \\
\hline Teen & 1. $2 E+00$ & $1.4 \mathrm{E}+00$ & $6.8 \mathrm{E}-02$ & $2.7 E+00$ & $2.1 \mathrm{E}+00$ \\
\hline Adult & $1.1 E+01$ & 1. $3 E+01$ & 4. $7 E-01$ & $1.8 \mathrm{E}+01$ & $1.2 \mathrm{E}+01$ \\
\hline TOTAL & $1.4 E+01$ & $1.5 \mathrm{E}+01$ & $6.4 E-01$ & $2.5 \mathrm{E}+01$ & $1.7 \mathrm{E}+01$ \\
\hline
\end{tabular}

Dose Commitments (person-rem) from Airborne Pathways

\begin{tabular}{|c|c|c|c|c|c|c|}
\hline & Total Body & GI-LLI & Thyroid & Bone & Liver & Lung \\
\hline Inf ant & $1.2 \mathrm{E}-04$ & $1.2 \mathrm{E}-04$ & $\overline{1.3 \mathrm{E}-04}$ & $1.2 E-04$ & $\overline{1.2 E-04}$ & $1.5 \mathrm{E}-04$ \\
\hline Child & 1. $3 E-03$ & $1.3 \mathrm{E}-03$ & $1.4 E-03$ & $1.5 \mathrm{E}-03$ & 1. $3 \mathrm{E}-03$ & $1.8 \mathrm{E}-03$ \\
\hline Teen & $9.6 E-04$ & $9.4 E-04$ & $9.8 E-04$ & 1.0E-03 & $9.6 \mathrm{E}-04$ & $1.6 \mathrm{E}-03$ \\
\hline Adult & & & & $6.0 \mathrm{E}-$ & & $7.8 \mathrm{E}-03$ \\
\hline TOTAL & $8.2 E-03$ & $8.0 E-03$ & $8.3 E-03$ & $8.6 \mathrm{E}-03$ & $8.1 E-03$ & $1.1 E-02$ \\
\hline
\end{tabular}

Production/consumption factors:

Produce: $<1$ Milk: $<1 \quad$ Meat: <1

FRACTION OF POPULATION RECEIVING AN INOICATEO AVERAGE TOTAL-BOOY DOSE COMMITMENT FROM AIRBORNE PATHWAYS

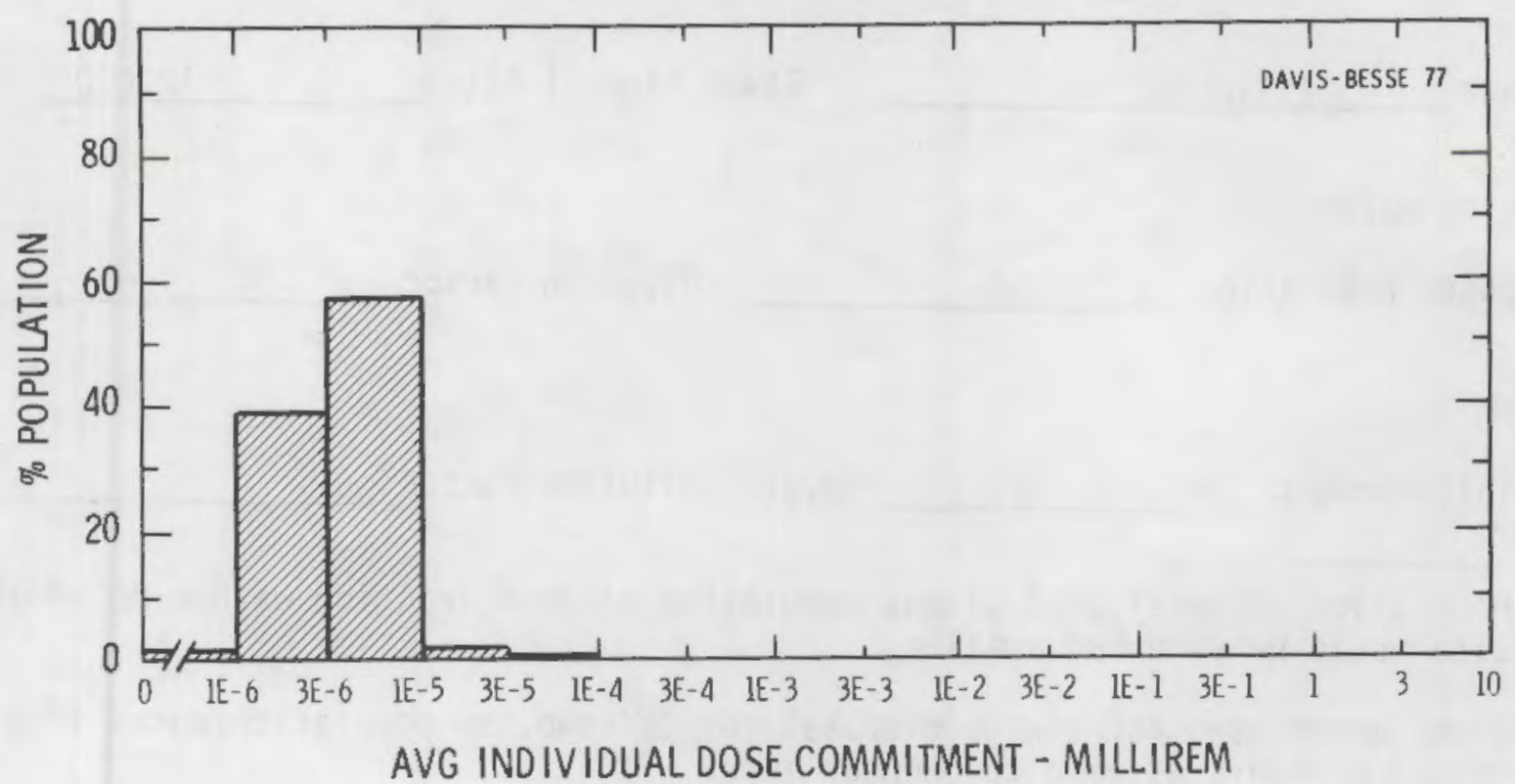


Site DRESDEN

Location $\mathrm{N}$ $41.3897^{\circ}$

Total Population Within 2-to-80-km Region
GRUNDY COUNTY, ILLINOIS

W $\quad 88.2714^{\circ}$

$6.4 \mathrm{E} 6$

Major Metropolitan Centers Within Region

Center

Chicago SMSA

Gary-Hammond-E. Chicago-SMSA

joliet

Aurora

Elgin
Population

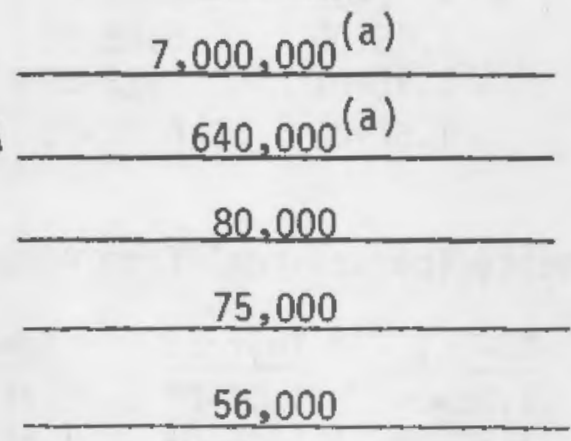

Location

\begin{tabular}{cc}
$72 \mathrm{~km}$ & NE \\
\hline $80 \mathrm{~km}$ & ENE \\
\hline $24 \mathrm{~km}$ & $\mathrm{NE}$ \\
\hline $43 \mathrm{~km}$ & $\mathrm{~N}$ \\
\hline $74 \mathrm{~km}$ & $\mathrm{~N}$ \\
\hline
\end{tabular}

Average Annual State Próduction of Crops and Animal Products in $80-\mathrm{km}$ Radius Circle Veg $1.1 \mathrm{E} 8$ _ $\mathrm{kg}$ Milk $1.8 \mathrm{E} 8{ }^{\ell}$ Meat $1.9 \mathrm{E} 8$

Regional Productivity Factor

Animal Grazing Factor

Location of Meteorological Station Site

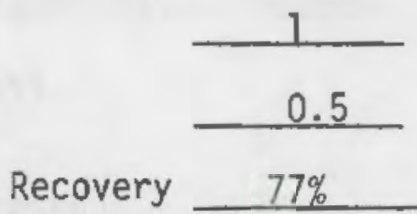

Period of Record

1 JAN $74=31$ JAN 75

Average

Ilinois

River Flow at Site

$12,000 \mathrm{ft}^{3} / \mathrm{s}$

Drinking Water

Exposed Population

(b)

Dilution Factor

Fish

Edible Harvest

(b) $\mathrm{kg} / \mathrm{yr}$ Dilution Factor

(a) Population of total SMSA given; population of SMSA fraction within $80 \mathrm{~km}$ of site would be somewhat smaller.

(b) River water used for sewage disposal for Chicago, so population doses from liquid pathways assumed to be near zero. 


\begin{abstract}
POPULATION DOSE-COMMITMENT ESTIMATES AND AVERAGE INDIVIDUAL DOSE-COMMITMENT HISTOGRAM FOR DRESDEN 1, $2 \& 3$
\end{abstract}

\begin{tabular}{|c|c|c|c|c|c|}
\hline & Total Body & GI-LLI & Thyroid & Bone & Liver \\
\hline $\begin{array}{l}\text { Infant } \\
\text { Child } \\
\text { Teen } \\
\text { Adult }\end{array}$ & $\begin{array}{l}0.0 \mathrm{E}+00 \\
1.4 \mathrm{E}+00 \\
2.6 \mathrm{E}+00 \\
2.8 \mathrm{E}+01\end{array}$ & $\begin{array}{l}0.0 \mathrm{E}+00 \\
3.3 \mathrm{E}-01 \\
6.9 \mathrm{E}-01 \\
6.0 \mathrm{E}+00\end{array}$ & $\begin{array}{l}0.0 \mathrm{E}+00 \\
2.6 \mathrm{E}-01 \\
1.9 \mathrm{E}-01 \\
1.2 \mathrm{E}+00\end{array}$ & $\begin{array}{l}0.0 \mathrm{E}+00 \\
7.7 \mathrm{E}+00 \\
4.7 \mathrm{E}+00 \\
2.7 \mathrm{E}+01\end{array}$ & $\begin{array}{l}0.0 \mathrm{E}+00 \\
7.9 \mathrm{E}+00 \\
6.6 \mathrm{E}+00 \\
3.9 \mathrm{E}+01\end{array}$ \\
\hline TOTAL & $3.2 E+01$ & $7.0 \mathrm{E}+00$ & 1. $7 E+00$ & 4. $0 E+01$ & $5.4 E+01$ \\
\hline
\end{tabular}

Dose Commitments (person-rem) from Airborne Pathways

\begin{tabular}{|c|c|c|c|c|c|c|}
\hline & Total Body & GI-LLI & Thyroid & Bone & Liver & Lung \\
\hline $\begin{array}{l}\text { Infant } \\
\text { Child } \\
\text { Teen } \\
\text { Adult }\end{array}$ & $\begin{array}{l}2.5 \mathrm{E}+00 \\
2.8 \mathrm{E}+01 \\
2.1 \mathrm{E}+01 \\
1.2 \mathrm{E}+02\end{array}$ & $\begin{array}{l}2.5 \mathrm{E}+00 \\
2.8 \mathrm{E}+01 \\
2.1 \mathrm{E}+01 \\
1.3 \mathrm{E}+02\end{array}$ & $\begin{array}{l}7.9 \mathrm{E}+00 \\
7.8 \mathrm{E}+01 \\
4.5 \mathrm{E}+01 \\
2.3 \mathrm{E}+02\end{array}$ & $\begin{array}{l}2.6 \mathrm{E}+00 \\
3.0 \mathrm{E}+01 \\
2.1 \mathrm{E}+01 \\
1.3 \mathrm{E}+02\end{array}$ & $\begin{array}{l}2.5 \mathrm{E}+00 \\
2.8 \mathrm{E}+01 \\
2.1 \mathrm{E}+01 \\
1.2 \mathrm{E}+02\end{array}$ & $\begin{array}{l}2.7 \mathrm{E}+00 \\
3.0 \mathrm{E}+01 \\
2.3 \mathrm{E}+01 \\
1.3 \mathrm{E}+02\end{array}$ \\
\hline TOTAL & $1.8 \mathrm{E}+02$ & $1.8 \mathrm{E}+02$ & $3.6 \mathrm{E}+02$ & $1.8 \mathrm{E}+02$ & $1.8 \mathrm{E}+02$ & $1.9 E+02$ \\
\hline
\end{tabular}

Production/consumption factors:

Produce: $<1$ M.ilk: $<1 \quad$ Meat: <1

FRACTION OF POPULATION RECEIVING AN INDICATED AVERAGE

TOTAL-BODY DOSE COMMITMENT FROM AIRBORNE PATHWAYS

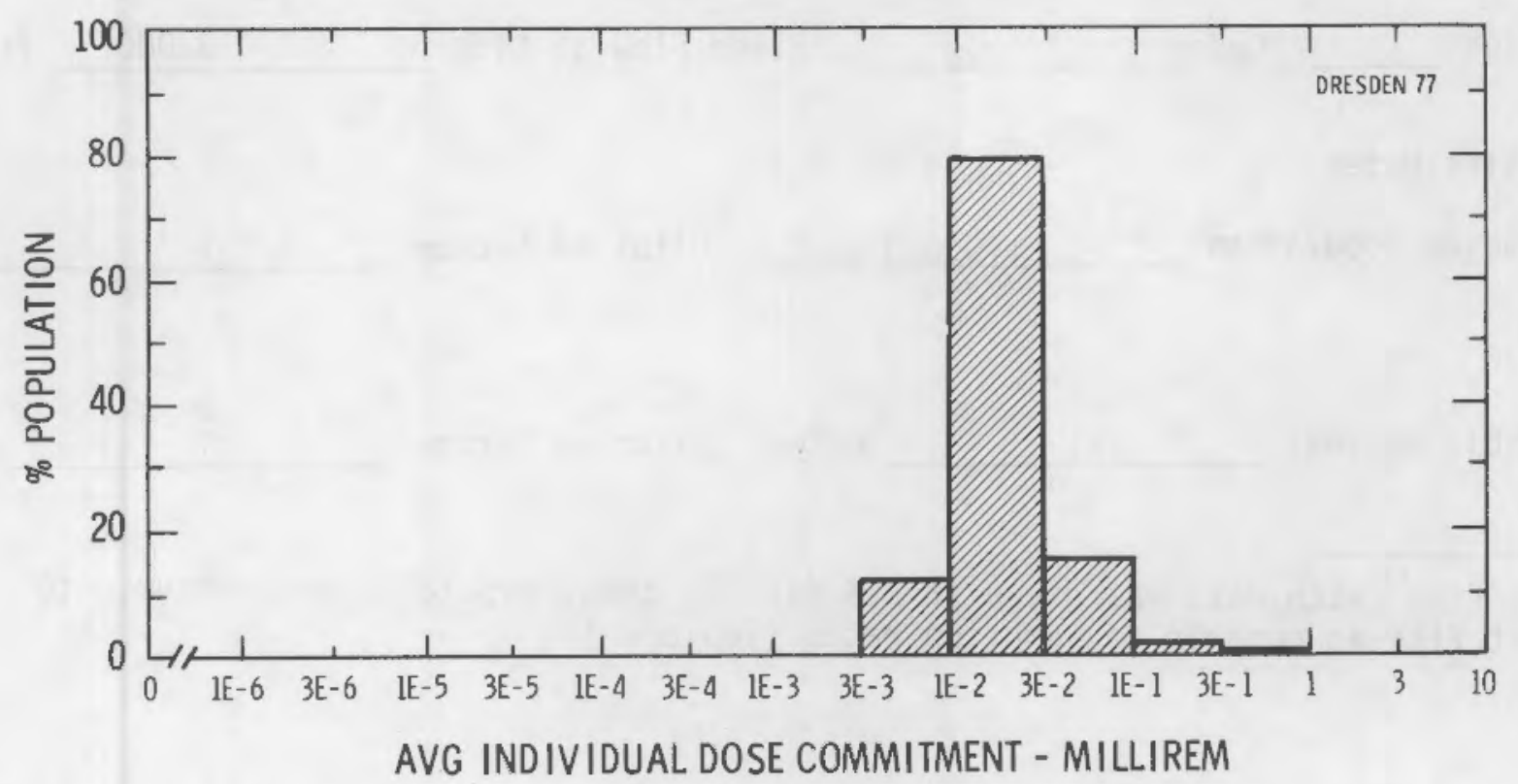


Site DUANE ARNOLD

Location

N

$42.0981^{\circ}$

Total Population Within 2-to-80-km Region
CEDAR RAPIDS, IOWA

W $91.7725^{\circ}$

Major Metropolitan Centers Within Region

$\frac{\frac{\text { Center }}{\text { Cedar Rapids }}}{\text { Waterloo }}$

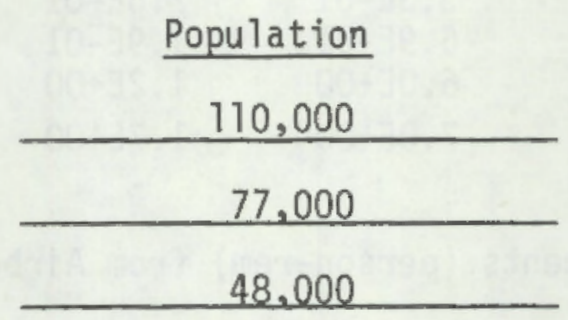

\begin{tabular}{cc}
$\frac{\text { Location }}{16 \mathrm{~km}}$ & SE \\
\hline $66 \mathrm{~km}$ & $\mathrm{NW}$ \\
\hline $53 \mathrm{~km}$ & SSE \\
\hline
\end{tabular}

Average Annual State Production of Crops and Animal Products in $80-\mathrm{km}$ Radius Circle

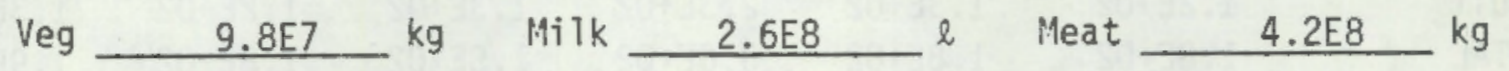

Regional Productivity Factor

$\frac{1}{0.5}$

Animal Grazing Factor Recovery $57 \%$

Location of Meteorological Station Site Period of Record 1 FEB 71 - 31 DEC 75

Average Cedar River Flow at Site $3,000 \quad \mathrm{ft}^{3} / \mathrm{s}$ Drinking Water Exposed Population 110,000 Dilution Factor 1

Fish

Edible Harvest (a) $\mathrm{kg} / \mathrm{yr}$ Dilution Factor 1

(a) No fish catch data was given in FES (1973); thus, population was assumed to eat fish at generic consumption rates (Table A-1). 
POPULATION OOSE-COMMITMENT ESTIMATES AND AVERAGE INDIVIDUAL DOSE-COMMITMENT HISTOGRAM FOR OUANE ARNOLO

Oose Commitments (person-rem) from Liquid Pathways

Inf ant

Child

Teen

Adult

TOTAL

\section{Inf ant}

Child

Teen

Adult

TOTAL

$\frac{G I-L L I}{8.8 E-06}$
$2.3 E-04$
$3.1 E-04$
$2.8 E-03$
$3.3 E-03$

Thyroid

1.2E-04

1.2E -03

5. $0 \mathrm{E}-04$

3. $5 \mathrm{E}-03$

$5.3 \mathrm{E}-03$
Bone

5.2E-06

9.6E-04

$5.9 \mathrm{E}-04$

3. $7 \mathrm{E}-03$

$5.2 \mathrm{E}-03$
Liver

9.6E-06

$1.5 \mathrm{E}-03$

1. $3 \mathrm{E}-03$

7. $8 \mathrm{E}-03$

1.1E-02

Dose Commitments (person-rem) from Airborne Pathways

\begin{tabular}{|c|c|c|c|c|c|}
\hline Total Body & GI-LLI & Thyroid & Bone & Liver & Lung \\
\hline $\begin{array}{l}4.3 \mathrm{E}-03 \\
4.8 \mathrm{E}-02 \\
3.5 \mathrm{E}-02 \\
2.1 \mathrm{E}-01\end{array}$ & $\begin{array}{l}4.3 \mathrm{E}-03 \\
4.8 \mathrm{E}-02 \\
3.5 \mathrm{E}-02 \\
2.1 \mathrm{E}-01\end{array}$ & $\begin{array}{l}9.1 \mathrm{E}-03 \\
9.7 \mathrm{E}-02 \\
5.5 \mathrm{E}-02 \\
2.9 \mathrm{E}-01\end{array}$ & $\begin{array}{l}4.3 \mathrm{E}-03 \\
4.8 \mathrm{E}-02 \\
3.5 \mathrm{E}-02 \\
2.1 \mathrm{E}-01\end{array}$ & $\begin{array}{l}4.3 \mathrm{E}-03 \\
4.9 \mathrm{E}-02 \\
3.5 \mathrm{E}-02 \\
2.1 \mathrm{E}-01\end{array}$ & $\begin{array}{l}\overline{4.4 \mathrm{E}-03} \\
5.0 \mathrm{E}-02 \\
3.7 \mathrm{E}-02 \\
2.2 \mathrm{E}-01\end{array}$ \\
\hline $3.0 \mathrm{E}-01$ & 3. $0 \mathrm{E}-01$ & $4.5 \mathrm{E}-01$ & 3. $0 \mathrm{E}-01$ & $3.0 \mathrm{E}-01$ & $3.1 E-01$ \\
\hline
\end{tabular}

Production/consumption factors:

Produce: $<1$

Milk: 3.4

Meat: 9.2

FRACTION OF POPULATION RECEIVING AN INOICATED AVERAGE TOTAL-BODY DOSE COMMITMENT FROM AIRBORNE PATHWAYS

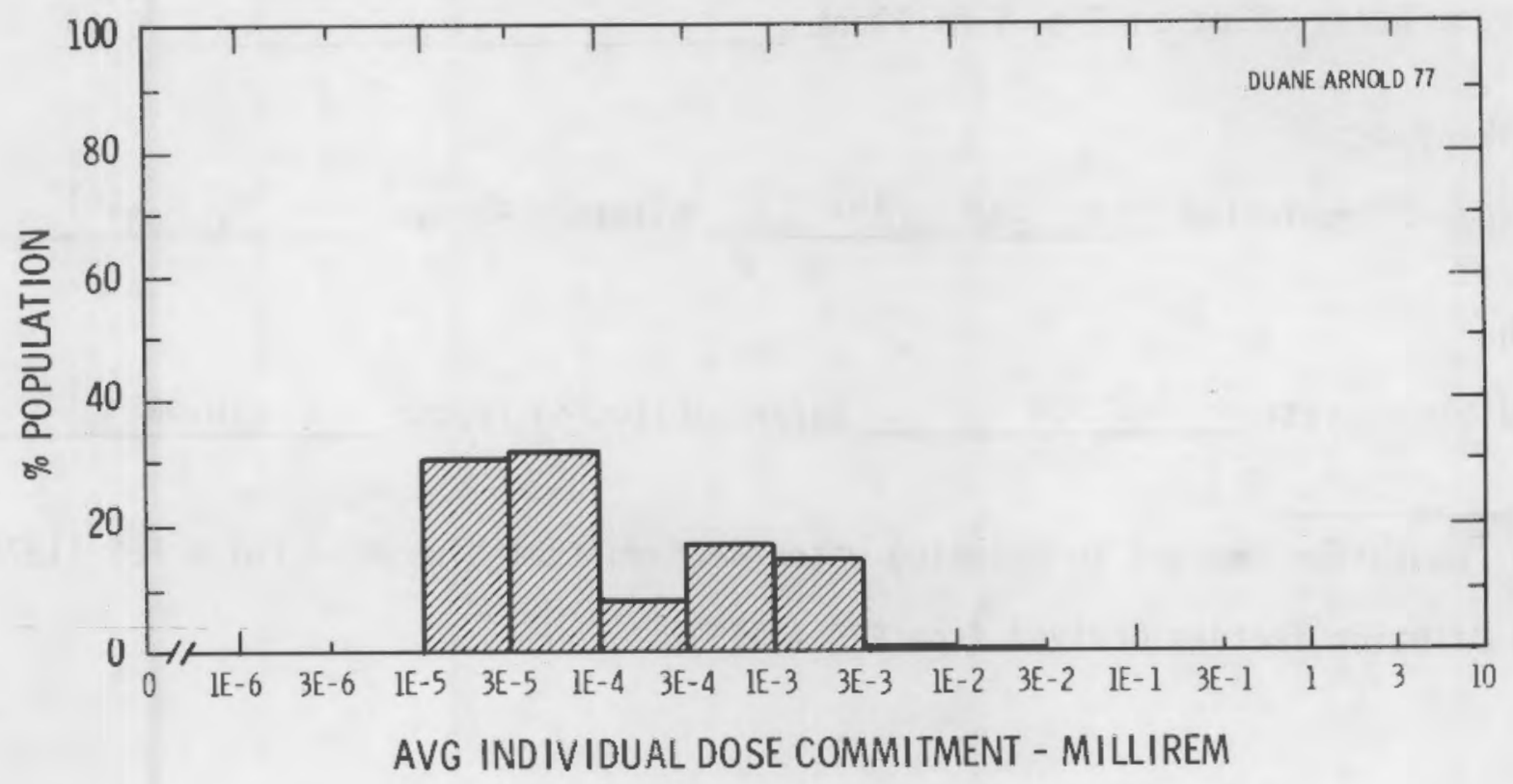


Site J.A. FITZPATRICK

Location $\mathrm{N}$ $43.5217^{\circ}$

Total Population Within 2-to-80-km Region
OSWEGO, NEW YORK

$76.3980^{\circ}$

Major Metropolitan Centers Within Region

\begin{tabular}{l} 
Syracuse SMSA \\
\hline Rome \\
\hline Auburn \\
\hline
\end{tabular}

$\begin{array}{r}\frac{\text { Population }}{630,000} \\ \hline 50,000 \\ \hline 34.000 \\ \hline\end{array}$

\begin{tabular}{cc}
\multicolumn{2}{c}{ Location } \\
$54 \mathrm{~km}$ & SSE \\
\hline $80 \mathrm{~km}$ & ESE \\
\hline $66 \mathrm{~km}$ & SSW \\
\hline
\end{tabular}

Average Annual State Production of Crops and Animal Products in $80-\mathrm{km}$ Radius Circle Veg $7.6 \mathrm{E} 7 \quad \mathrm{~kg} M i 1 \mathrm{k}+7.0 \mathrm{E} 8{ }^{\ell}$ Meat $3.3 \mathrm{EF}$

Regional Productivity Factor

0.7

Animal Grazing Factor

0.5

Location of Meteorological Station Nine Mile Point Site

Recovery $97 \%$

Period of Record

1 JAN $74-31$ DEC 75

Average Water Dilution Flow from Plant $610 \mathrm{ft}^{3} / \mathrm{s}$

Drinking Water

Exposed Population $540.000^{(a)}$

Dilution Factor $0.003^{(b)}$

Fish

Edible Harvest 7.3E5 $\mathrm{kg} / \mathrm{yr}$ Dilution Factor $0.005^{(b)}$

(a) Population exposed to drinking water derived from Nine Mile Point FES (1974).

(b) Dilution factors derived from FES (1973). 


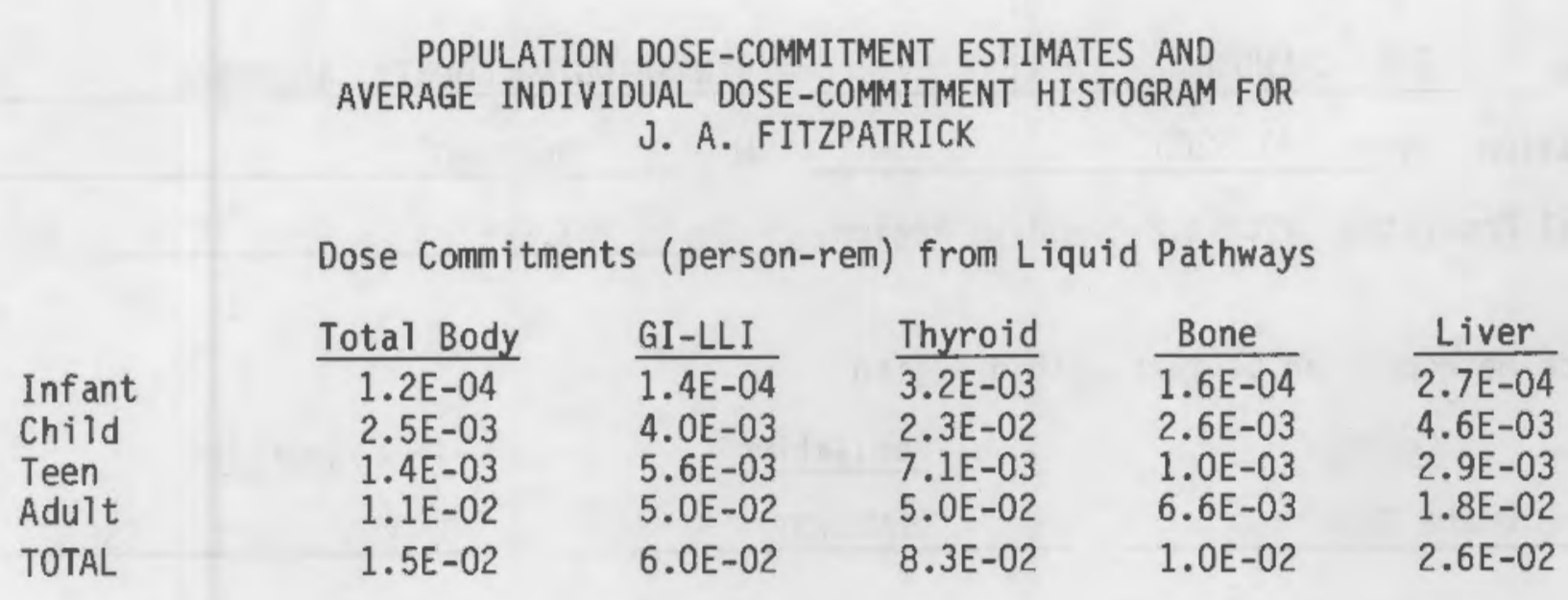

Dose Commitments (person-rem) from Airborne Pathways

\begin{tabular}{|c|c|c|c|c|c|c|}
\hline & Total Body & GI-LLI & Thyroid & Bone & Liver & Lung \\
\hline $\begin{array}{l}\text { Infant } \\
\text { Child } \\
\text { Teen } \\
\text { Adult }\end{array}$ & $\begin{array}{l}7.9 \mathrm{E}-03 \\
8.8 \mathrm{E}-02 \\
6.4 \mathrm{E}-02 \\
3.8 \mathrm{E}-01\end{array}$ & $\begin{array}{l}7.8 \mathrm{E}-03 \\
8.7 \mathrm{E}-02 \\
6.4 \mathrm{E}-02 \\
3.9 \mathrm{E}-01\end{array}$ & $\begin{array}{l}8.9 \mathrm{E}-02 \\
6.3 \mathrm{E}-01 \\
2.6 \mathrm{E}-01 \\
1.0 \mathrm{E}+00\end{array}$ & $\begin{array}{l}8.0 \mathrm{E}-03 \\
8.9 \mathrm{E}-02 \\
6.4 \mathrm{E}-02 \\
3.8 \mathrm{E}-01\end{array}$ & $\begin{array}{l}8.1 \mathrm{E}-03 \\
8.9 \mathrm{E}-02 \\
6.4 \mathrm{E}-02 \\
3.9 \mathrm{E}-01\end{array}$ & $\begin{array}{l}8.1 \mathrm{E}-03 \\
9.1 \mathrm{E}-02 \\
6.9 \mathrm{E}-02 \\
4.0 \mathrm{E}-01\end{array}$ \\
\hline TOTAL & $5.4 \mathrm{E}-01$ & $5.4 \mathrm{E}-01$ & $2.0 E+00$ & $5.4 \mathrm{E}-01$ & $5.5 E-01$ & $5.7 \mathrm{E}-01$ \\
\hline
\end{tabular}

Production/consumption factors:

Produce: <1 Milk: $4.5 \quad$ Meat: <1

FRACTION OF POPULATION RECEIVING AN INDICATED AVERAGE

TOTAL-BODY OOSE COMMITMENT FROM AIRBORNE PATHWAYS

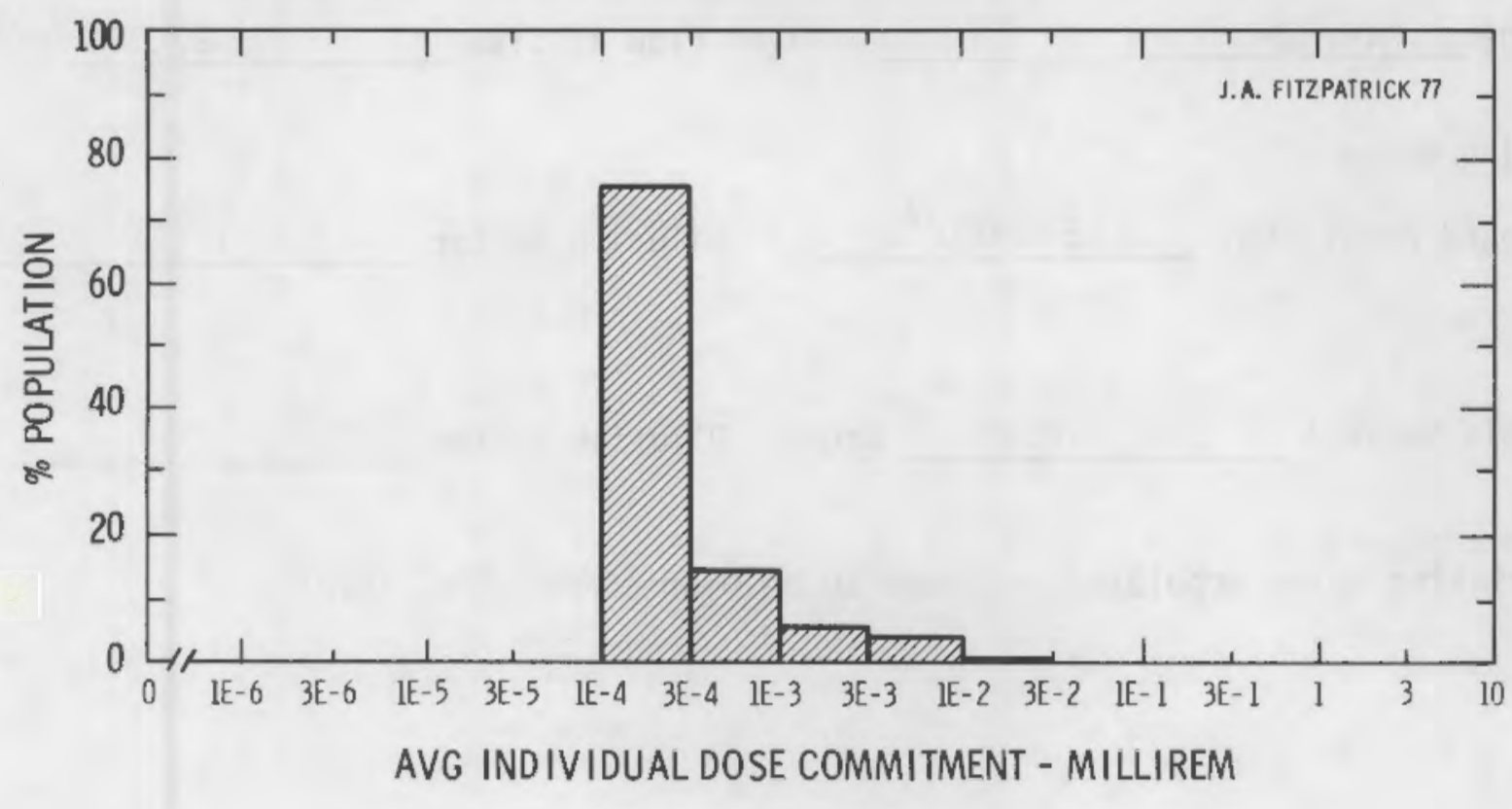


Site FORT CALHOUN

Location $\mathrm{N}$

Total Population Within 2-to-80-km Region
WASHINGTON COUNTY, NEBRASKA

w

$96.0780^{\circ}$

Major Metropolitan Centers Within Region

Center

Omaha SMSA
Population

570,000
Location

$32 \mathrm{~km}$ SSE

Average Annual State Production of Crops and Animal Products in 80-km Radius Circle

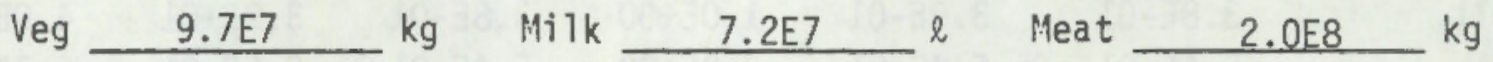

Regional Productivity Factor

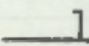

Animal Grazing Factor

0.5

Location of Meteorological Station Site Recovery $98 \%$

Period of Record

1 JAN $74-31$ OEC 74

Average Missouri

River Flow at site

$27,000 \mathrm{ft}^{3} / \mathrm{s}$

Drinking Water

Exposed Population

$570,000^{(a)}$

Dilution Factor 1

Fish

Edible Harvest 1.0E4 $\mathrm{kg} / \mathrm{yr}$ Dilution Factor 1

(a) Drinking water population assumed to be Omaha SMSA (FES, 1972). 


\section{POPULATION DOSE-COMMITMENT ESTIMATES AND AVERAGE INDIVIDUAL DOSE-COMMITMENT HISTOGRAM FDR FORT CALHOUN}

\begin{tabular}{|c|c|c|c|c|c|}
\hline & Total Body & GI-LLI & Thyroid & Bone & Liver \\
\hline $\begin{array}{l}\text { Inf ant } \\
\text { Child } \\
\text { Teen } \\
\text { Adult }\end{array}$ & $\begin{array}{l}3.5 \mathrm{E}-03 \\
4.5 \mathrm{E}-02 \\
2.3 \mathrm{E}-02 \\
2.2 \mathrm{E}-01\end{array}$ & $\begin{array}{l}2.9 \mathrm{E}-03 \\
3.4 \mathrm{E}-02 \\
1.4 \mathrm{E}-02 \\
1.2 \mathrm{E}-01\end{array}$ & $\begin{array}{l}2.6 \mathrm{E}-02 \\
2.0 \mathrm{E}-01 \\
6.2 \mathrm{E}-02 \\
4.6 \mathrm{E}-01\end{array}$ & $\begin{array}{l}5.0 \mathrm{E}-03 \\
5.6 \mathrm{E}-02 \\
1.6 \mathrm{E}-02 \\
9.8 \mathrm{E}-02\end{array}$ & $\begin{array}{l}9.7 \mathrm{E}-03 \\
9.6 \mathrm{E}-02 \\
3.7 \mathrm{E}-02 \\
2.6 \mathrm{E}-01\end{array}$ \\
\hline TOTAL & $2.9 \mathrm{E}-01$ & 1. $7 \mathrm{E}-01$ & $7.4 \mathrm{E}-01$ & $1.7 \mathrm{E}-01$ & 4.0E-01 \\
\hline
\end{tabular}

Dose Commitments (person-rem) from Liquid Pathways

Dose Cormitments (person-rem) from Airborne Pathways

\begin{tabular}{|c|c|c|c|c|c|c|}
\hline & Total Body & GI-LLI & Thyroid & Bone & Liver & Lung \\
\hline $\begin{array}{l}\text { Inf ant } \\
\text { Child } \\
\text { Teen } \\
\text { Adult }\end{array}$ & $\begin{array}{l}6.3 \mathrm{E}-04 \\
7.1 \mathrm{E}-03 \\
5.2 \mathrm{E}-03 \\
3.1 \mathrm{E}-02\end{array}$ & $\begin{array}{l}6.3 \mathrm{E}-04 \\
7.0 \mathrm{E}-03 \\
5.1 \mathrm{E}-03 \\
3.1 \mathrm{E}-02\end{array}$ & $\begin{array}{l}5.4 \mathrm{E}-03 \\
5.5 \mathrm{E}-02 \\
2.4 \mathrm{E}-02 \\
9.9 \mathrm{E}-02\end{array}$ & $\begin{array}{l}6.3 \mathrm{E}-04 \\
7.1 \mathrm{E}-03 \\
5.1 \mathrm{E}-03 \\
3.1 \mathrm{E}-02\end{array}$ & $\begin{array}{l}6.4 \mathrm{E}-04 \\
7.2 \mathrm{E}-03 \\
5.2 \mathrm{E}-03 \\
3.1 \mathrm{E}-02\end{array}$ & $\begin{array}{l}6.7 \mathrm{E}-04 \\
7.7 \mathrm{E}-03 \\
6.0 \mathrm{E}-03 \\
3.4 \mathrm{E}-02\end{array}$ \\
\hline TOTAL & $4.4 \mathrm{E}-02$ & $4.4 \mathrm{E}-02$ & $1.8 \mathrm{E}-01$ & 4. $4 \mathrm{E}-02$ & 4. $4 \mathrm{E}-02$ & $4.8 \mathrm{E}-02$ \\
\hline
\end{tabular}

Production/consumption factors:

Produce: $<1$ Milk: $<1$ Meat: 3.3

FRACTION OF POPULATION RECEIVING AN INDICATED AVERAGE

TOTAL-BODY DOSE COMMITMENT FROM AIRBORNE PATHWAYS

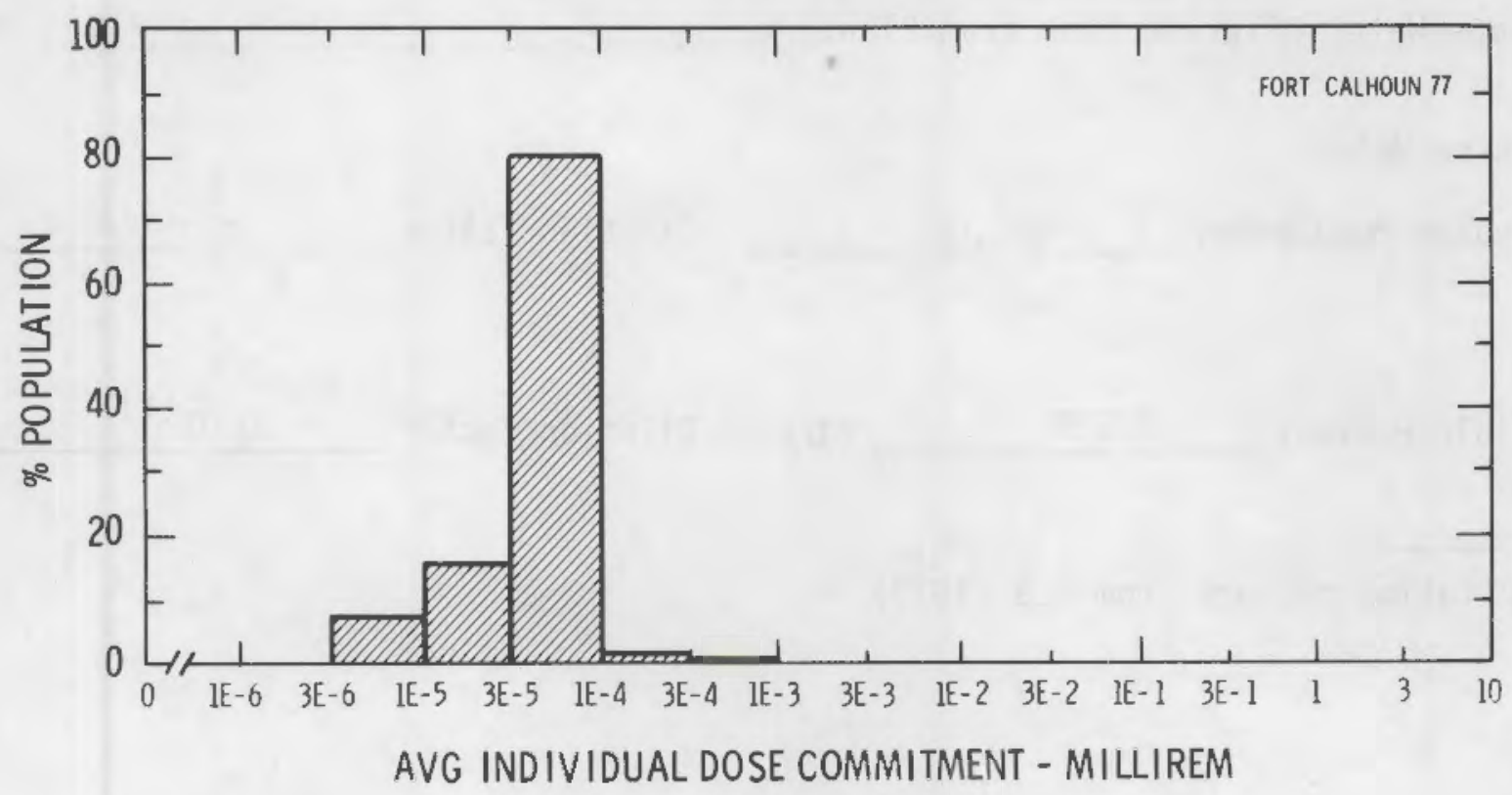


Site

R. E. GINNA

Location $\mathrm{N}$ $43.2772^{\circ}$

Total Population Within 2-to-80-km Region
ONTARIO, NEW YORK

W $77.3081^{\circ}$

Major Metropolitan Centers Within Region

\begin{tabular}{l}
$\frac{\text { Center }}{\text { Rochester SMSA }}$ \\
\hline Auburn \\
\hline Oswego
\end{tabular}

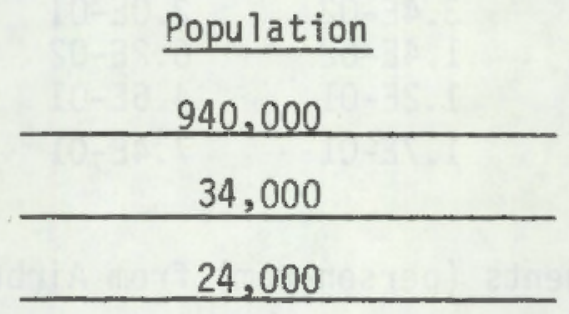

\begin{aligned} &$\frac{\text { Location }}{34 \mathrm{~km}}$ WSW \\ & \hline $64 \mathrm{~km}$ SE \\ & \hline $56 \mathrm{~km}$ ENE \\ & \hline\end{aligned}

Average Annual State Production of Crops and Animal Products in $80-\mathrm{km}$ Radius Circle

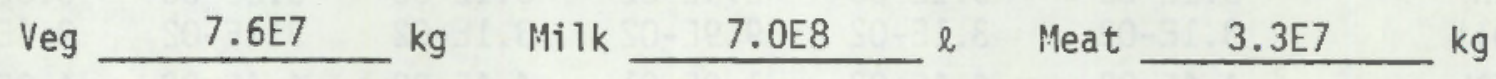

Regional Productivity Factor

Animal Grazing Factor

0.6

Location of Meteorological Station Site 0.5

Period of Record

1 JAN $66-31$ DEC 67

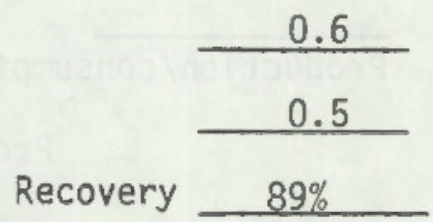

Average Water Dilution Flow from Plant $710 \mathrm{ft} / \mathrm{s}$

Drinking Water

Exposed Population 540,000

Fish

Edible Harvest 7.3E5 $\mathrm{kg} / \mathrm{yr}$ Dilution Factor $0.01^{(a)}$

(a) Dilution factors from FES (1973). 
POPULATION DOSE-COMMITMENT ESTIMATES AND AVERAGE INDIVIDUAL DOSE-COMMITMENT HISTOGRAM FOR

R. E. GINNA

Dose Commitments (person-rem) from Liquid Pathways

Inf ant

Child

Teen

Adult

TOTAL $\frac{\text { Total Body }}{7.1 \mathrm{E}-04}$

$9.8 \mathrm{E}-03$

$6.5 \mathrm{E}-03$

$6.5 \mathrm{E}-02$

$8.2 \mathrm{E}-02$
$\frac{\mathrm{GI}-\mathrm{LLI}}{6.9 \mathrm{E}-04}$

$8.6 \mathrm{E}-03$

$4.6 \mathrm{E}-03$

$3.9 \mathrm{E}-\mathrm{O} 2$

$5.3 \mathrm{E}-02$ $\frac{\text { Thyroid }}{1.3 \mathrm{E}-03}$

1.2E -02

4.3E-03

$3.4 \mathrm{E}-02$

$5.2 \mathrm{E}-02$ $\frac{\text { Bone }}{2.3 E-04}$

$1.2 \mathrm{E}-02$

$6.2 \mathrm{E}-03$

3.6E-02

$5.4 \mathrm{E}-02$ $\frac{\text { Liver }}{9.8 \mathrm{E}-04}$

2.0 -02

$1.2 \mathrm{E}-02$

$8.1 \mathrm{E}-02$

$1.1 \mathrm{E}-01$

Dose Commitments (person-rem) from Airborne Pathways

\begin{tabular}{|c|c|c|c|c|c|c|}
\hline & Total Body & GI-LLI & Thyroid & Bone & Liver & Lung \\
\hline $\begin{array}{l}\text { Infant } \\
\text { Child } \\
\text { Teen } \\
\text { Adult }\end{array}$ & $\begin{array}{l}8.2 E-04 \\
9.5 E-03 \\
6.6 E-03 \\
3.9 E-02\end{array}$ & $\begin{array}{l}8.0 \mathrm{E}-04 \\
9.4 \mathrm{E}-03 \\
6.6 \mathrm{E}-03 \\
3.8 \mathrm{E}-02\end{array}$ & $\begin{array}{l}1.4 \mathrm{E}-02 \\
9.1 \mathrm{E}-02 \\
3.8 \mathrm{E}-02 \\
1.4 \mathrm{E}-01\end{array}$ & $\begin{array}{l}6.6 \mathrm{E}-04 \\
7.1 \mathrm{E}-03 \\
5.1 \mathrm{E}-03 \\
3.1 \mathrm{E}-02\end{array}$ & $\begin{array}{l}8.5 \mathrm{E}-04 \\
9.7 \mathrm{E}-03 \\
6.7 \mathrm{E}-03 \\
3.9 \mathrm{E}-02\end{array}$ & $\begin{array}{l}8.6 \mathrm{E}-04 \\
1.0 \mathrm{E}-02 \\
7.6 \mathrm{E}-03 \\
4.2 \mathrm{E}-02\end{array}$ \\
\hline TOTAL & $5.6 E-02$ & $5.5 \mathrm{E}-02$ & 2. $9 \mathrm{E}-01$ & $4.4 \mathrm{E}-02$ & $5.6 \mathrm{E}-02$ & $6.0 \mathrm{E}-02$ \\
\hline
\end{tabular}

Production/consumption factors:

Produce: $<1$

Milk: 2.8

Meat: $<1$

FRACTION OF POPULATION RECEIVING AN INDICATED AVERAGE TOTAL-BODY DOSE COMMITMENT FROM AIRBORNE PATHWAYS

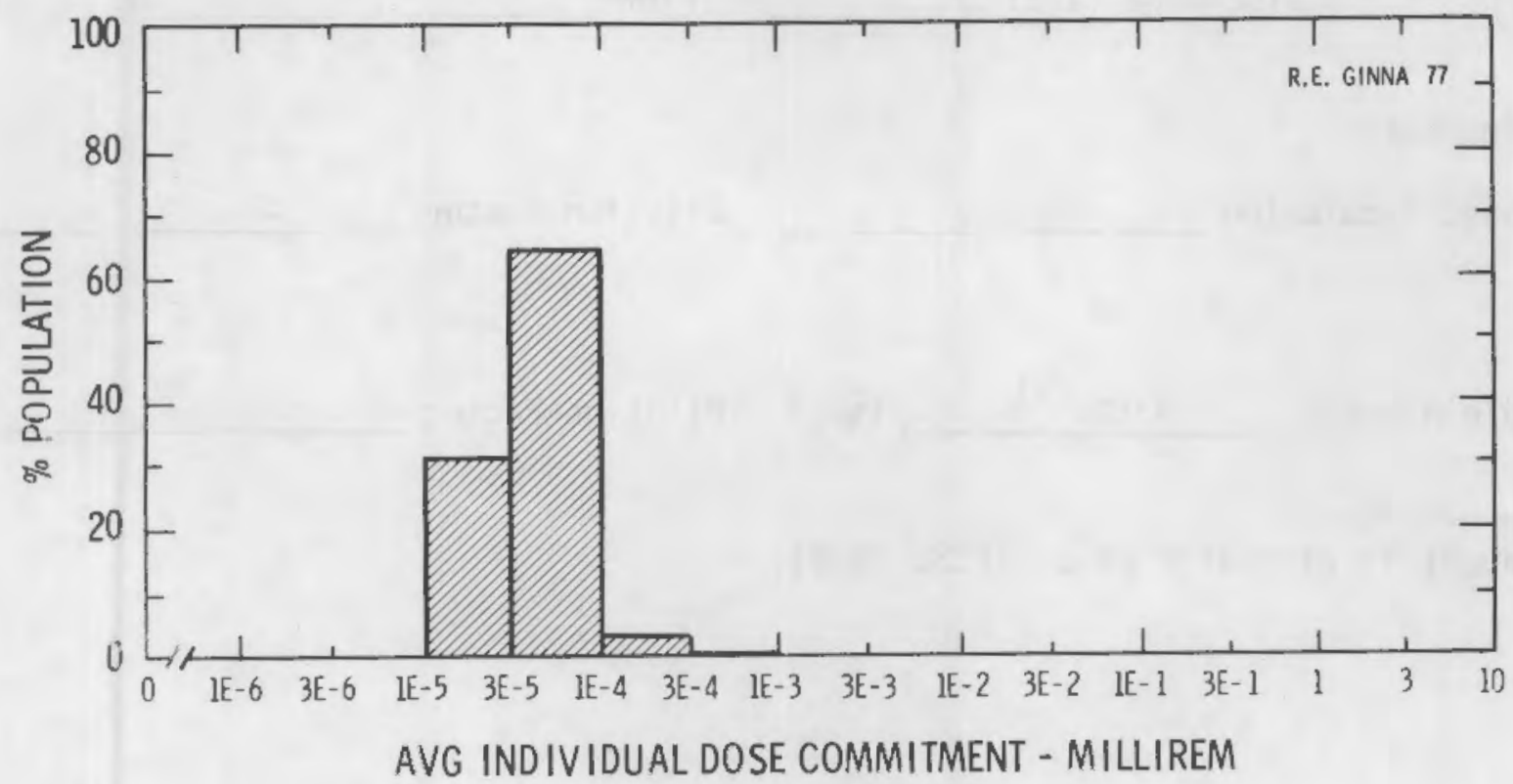


Site HADDAM NECK (CONN. YANKEE)

Location $N$ $41.4822^{\circ}$

Total Population Within 2-to-80-km Region
HADDAM NECK, CONNECTICUT

W $72.4989^{\circ}$

Major Metropolitan Centers Within Region

\begin{tabular}{l} 
Hartford SMSA \\
\hline New Haven SMSA \\
\hline Springfield SMSA \\
Waterbury \\
\hline
\end{tabular}

\begin{tabular}{c}
$\frac{\text { Population }}{830,000}$ \\
\hline 760,000 \\
\hline 600,000 \\
\hline 110,00 \\
\hline
\end{tabular}

\begin{tabular}{ll}
\multicolumn{2}{c}{ Location } \\
$37 \mathrm{~km}$ & $\mathrm{NW}$ \\
\hline $40 \mathrm{~km}$ & $\mathrm{WSW}$ \\
\hline $72 \mathrm{~km}$ & $\mathrm{~N}$ \\
\hline $47 \mathrm{~km}$ & $\mathrm{~W}$ \\
\hline
\end{tabular}

Average Annual State Production of Crops and Animal Products in $80-\mathrm{km}$ Radius Circle

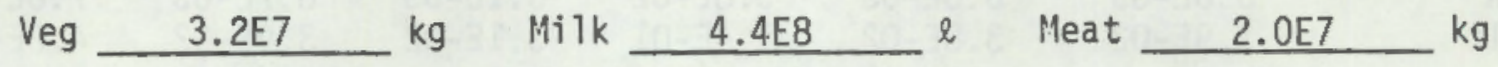

Regional Productivity Factor 0.7

Animal Grazing Factor

Location of Meteorological Station Site 0.6 Period of Record 1 JAN $75-31$ DEC 75

Average Discharge Canal

Flow $780-f t^{3} / 5$

Drinking Water

Exposed Population None

Dilution Factor

Fish

Edible Harvest $9.1 E 3^{(a)}$ $\mathrm{kg} / \mathrm{yr}$ Dilution Factor 1

(a) Caught in discharge canal (FES, 1973). 


\begin{abstract}
POPULATION OOSE-COMMITMENT ESTIMATES AND AVERAGE INDIVIOUAL OOSE-COMMITMENT HISTOGRAM FOR HAODAM NECK
\end{abstract}

Dose Commitments (person-rem) from Liquid Pathways

Inf ant

Child

Teen

Adult

TOTAL

\begin{tabular}{|c|c|c|c|c|}
\hline Total Body & GI-LLI & Thyroid & Bone & Liver \\
\hline $\begin{array}{l}0.0 \mathrm{E}+00 \\
1.0 \mathrm{E}-02 \\
1.9 \mathrm{E}-02 \\
2.0 \mathrm{E}-01\end{array}$ & $\begin{array}{l}0.0 \mathrm{E}+00 \\
7.1 \mathrm{E}-03 \\
1.4 \mathrm{E}-02 \\
1.2 \mathrm{E}-01\end{array}$ & $\begin{array}{l}0.0 \mathrm{E}+00 \\
4.0 \mathrm{E}-02 \\
2.9 \mathrm{E}-02 \\
1.9 \mathrm{E}-01\end{array}$ & $\begin{array}{l}0.0 \mathrm{E}+00 \\
4.8 \mathrm{E}-02 \\
2.9 \mathrm{E}-02 \\
1.7 \mathrm{E}-01\end{array}$ & $\begin{array}{l}0.0 \mathrm{E}+00 \\
5.4 \mathrm{E}-02 \\
4.6 \mathrm{E}-02 \\
4.7 \mathrm{E}-01\end{array}$ \\
\hline 2. $3 E-01$ & $1.4 \mathrm{E}-01$ & $2.6 \mathrm{E}-01$ & $2.4 \mathrm{E}-01$ & 3.7E-01 \\
\hline
\end{tabular}

Dose Commitments (person-rem) from Airborne Pathways

\begin{tabular}{|c|c|c|c|c|c|c|}
\hline & Total Body & GI-LLI & Thyroid & Bone & Liver & Lung \\
\hline $\begin{array}{l}\text { Infant } \\
\text { Child } \\
\text { Teen } \\
\text { Adult }\end{array}$ & $\begin{array}{l}1.3 \mathrm{E}-01 \\
8.4 \mathrm{E}-01 \\
2.9 \mathrm{E}-01 \\
9.8 \mathrm{E}-01\end{array}$ & $\begin{array}{l}1.3 E-01 \\
8.4 E-01 \\
2.9 E-01 \\
9.8 E-01\end{array}$ & $\begin{array}{l}1.3 E-01 \\
8.5 E-01 \\
2.9 E-01 \\
9.9 E-01\end{array}$ & $\begin{array}{l}5.8 \mathrm{E}-01 \\
3.9 \mathrm{E}+00 \\
1.2 \mathrm{E}+00 \\
3.4 \mathrm{E}+00\end{array}$ & $\begin{array}{l}1.3 \mathrm{E}-01 \\
8.4 \mathrm{E}-01 \\
2.9 \mathrm{E}-01 \\
9.8 \mathrm{E}-01\end{array}$ & $\begin{array}{l}\text { 1. } 3 E-01 \\
8.5 E-01 \\
3.0 E-01 \\
1.0 E+00\end{array}$ \\
\hline TOTAL & $2.2 E+00$ & $2.2 \mathrm{E}+00$ & 2. $3 \mathrm{E}+00$ & $9.1 E+00$ & $2.2 \mathrm{E}+00$ & $2.3 E+00$ \\
\hline
\end{tabular}

Production/consumption factors:

Produce: <1 Milk: <1 Meat: <1

FRACTION OF POPULATION RECEIVING AN INDICATEO AVERAGE

TOTAL-BODY DOSE COMMITMENT FROM AIRBORNE PATHWAYS

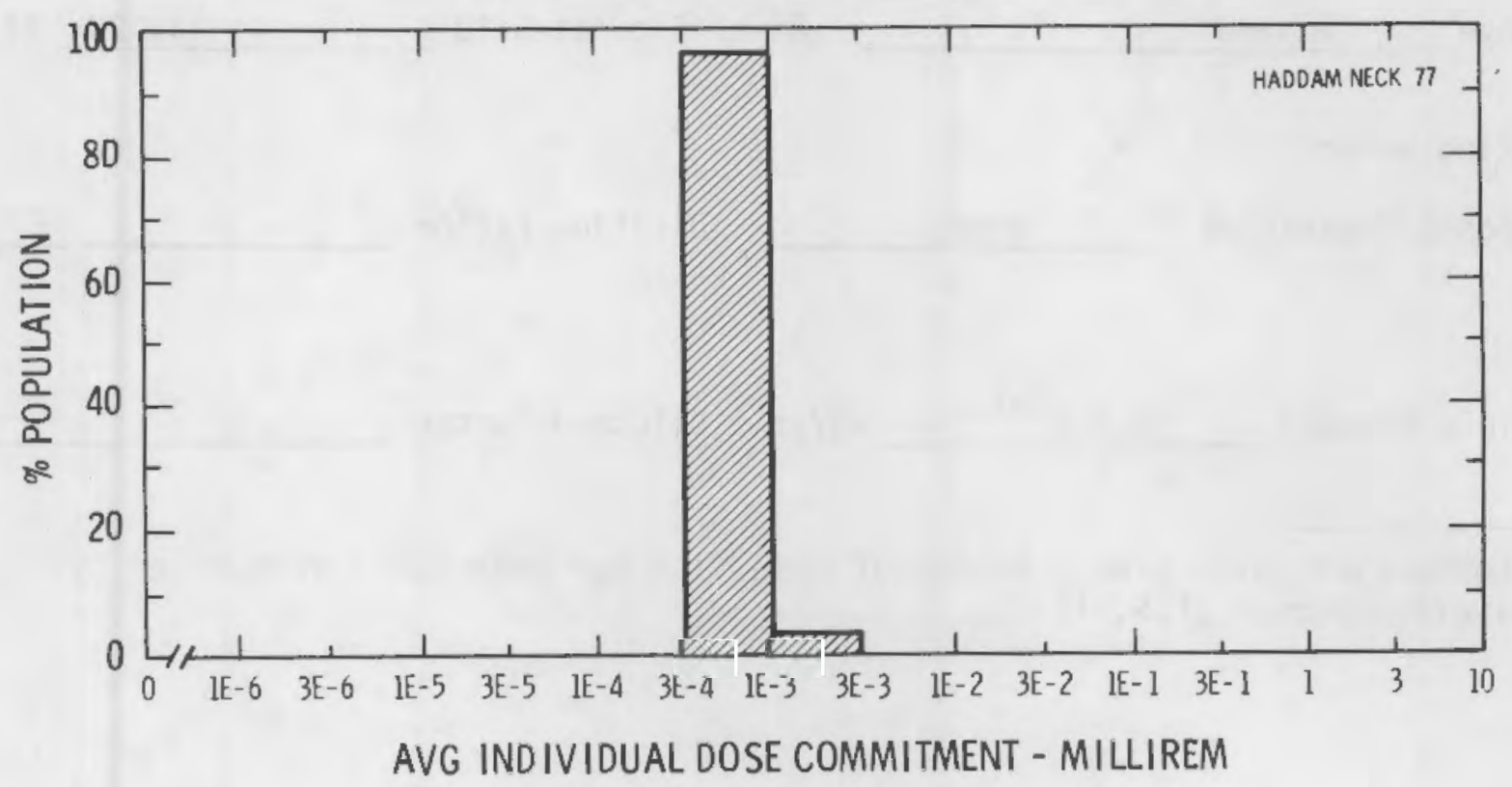


Site

E. I. HATCH

Location $\mathrm{N}$ $31.9344^{\circ}$

Total Population Within 2-to-80-km Region
BAXLEY, GEORGIA

$82.3444^{\circ}$

2.8E5

Major Metropolitan Centers Within Region

Center

Waycross
Population

21,000

\section{Location}

$80 \mathrm{~km}$

S

Average Annual State Production of Crops and Animal Products in $80-\mathrm{km}$ Radius Circle Veg 8.8E6 $\mathrm{kg}$ Milk $7.0 \mathrm{E} 7$ \& Meat 8.1E7 $\mathrm{kg}$

Regional Productivity Factor

Animal Grazing Factor

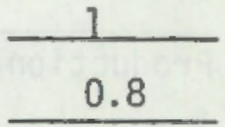

Location of Meteorological

Station Site

Recovery $87 \%$

Period of Record 1 JUN 70 - 31 AUG 74

Average A7 tamaha River Flow at Site $13,000 \mathrm{ft}^{3} / \mathrm{s}$

Drinking Water

Exposed Population None Dilution Factor

Fish

Edible Harvest $6.3 E 5^{(a)}$ $\mathrm{kg} / \mathrm{yr}$ Dilution Factor 1

(a) Commercial catch plus 3 pounds of game fish per year taken from river by average person (FES, 1972). 


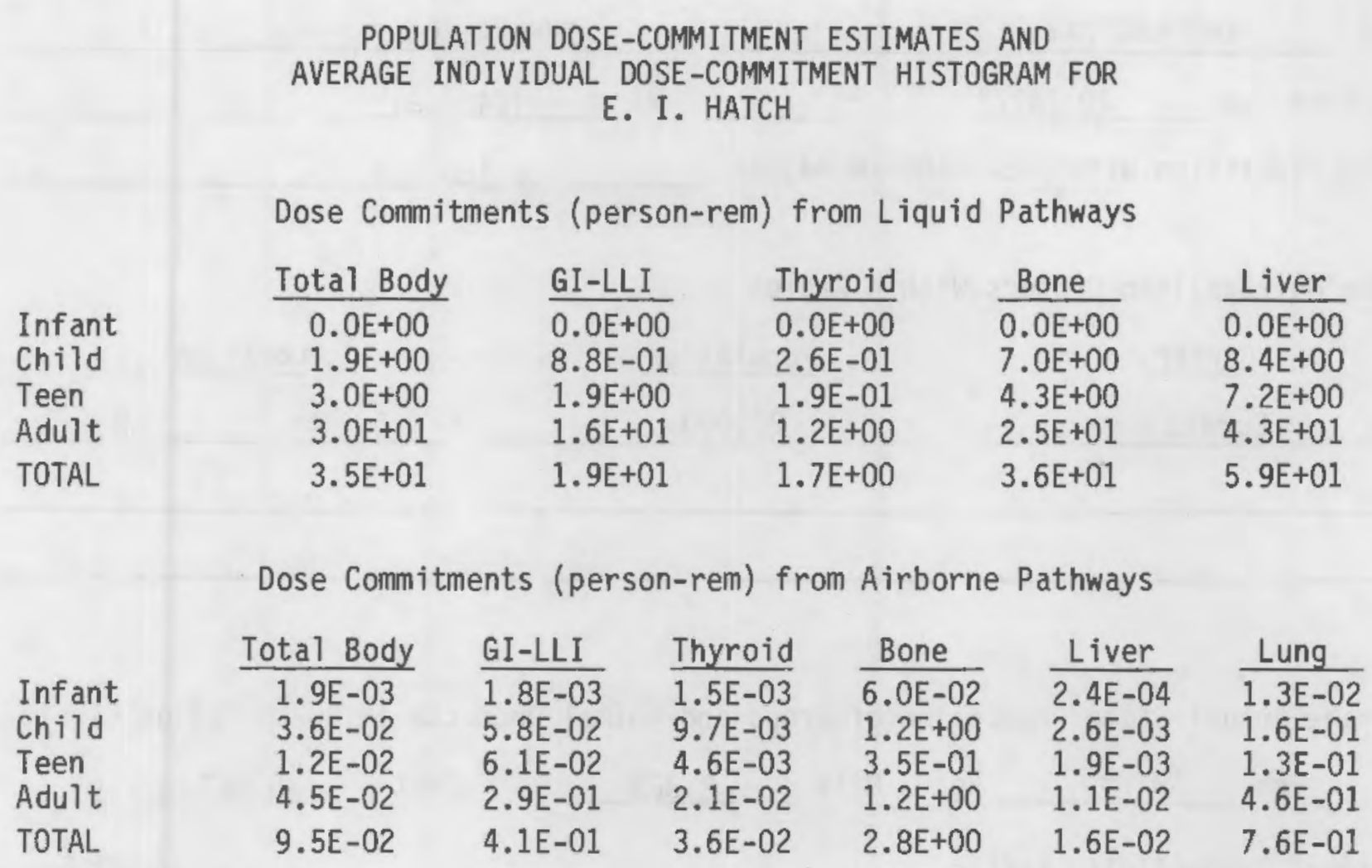

Production/consumption factors:

Produce: <1 Milk: $2.0 \quad$ Meat: 3.7

FRACTION OF POPULATION RECEIVING AN INDICATED AVERAGE TOTAL-BODY DOSE COMMITMENT FROM AIRBORNE PATHWAYS

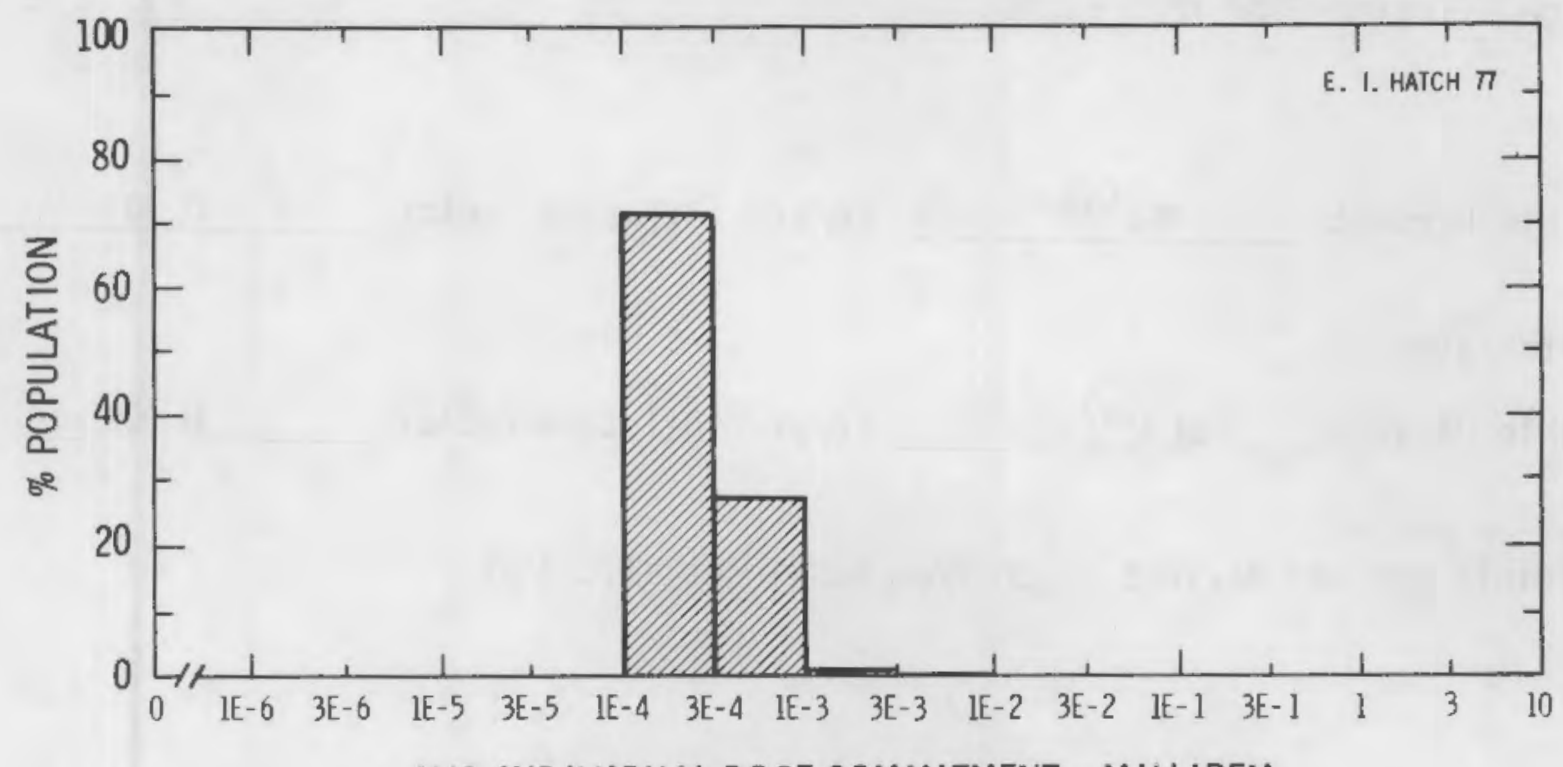

AVG INDIVIDUAL DOSE COMMITMENT - MILLIREM 
Site HUMBOLDT BAY

Location $\mathrm{N}$

$40.7417^{\circ}$

Tota 1 Population Within 2-to-80-km Region
EUREKA, CALIFORNIA

$124.2087^{\circ}$

Major Metropolitan Centers Within Region

Center

Eureka
Population

26,000

Location

$6.4 \mathrm{~km}$

N

Average Annual State Production of Crops and Animal Products in 80-km Radius Circle

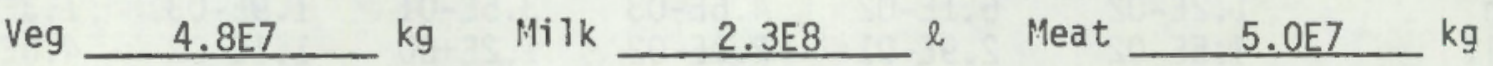

Regional Productivity Factor

$\frac{0.5}{1}$

Animal Grazing Factor

Location of Meteorological Station Site

Recovery $96 \%$

Period of Record 1 JAN 66 - 31 DEC 67

Average Dilution Flow from Plant $150 \mathrm{ft}^{3} / \mathrm{s}$

Fish

Edible Harvest $4 E 4^{(a)}$ $\mathrm{kg} / \mathrm{y}$ Dilution Factor 0.001

Invertebrates

Edible Harvest $5 E 3^{(a)}$ $\mathrm{kg} / \mathrm{yr}$ Dilution Factor 0.002

(a) Edible seafood harvest taken from Noskin et al., 1976. 


\begin{tabular}{|c|c|c|c|c|c|c|}
\hline & \multicolumn{5}{|c|}{$\begin{array}{l}\text { POPULATIDN DOSE-COMMITMENT ESTIMATES AND } \\
\text { AVERAGE INDIVIDUAL DOSE-COMMITMENT HISTOGRAM FOR } \\
\text { HUMBOLDT BAY }\end{array}$} & \multirow[b]{3}{*}{ Liver } \\
\hline & \multicolumn{5}{|c|}{ Dose Commitments (person-rem) from Liquid Pathways } & \\
\hline & Total Body & \multicolumn{2}{|c|}{ GI-LLI } & Thyroid & Bone & \\
\hline $\begin{array}{l}\text { Infant } \\
\text { Child } \\
\text { Teen } \\
\text { Adult }\end{array}$ & $\begin{array}{l}0.0 \mathrm{E}+00 \\
2.3 \mathrm{E}-04 \\
1.9 \mathrm{E}-04 \\
1.4 \mathrm{E}-03\end{array}$ & $\begin{array}{l}0.0 \mathrm{E} \\
1.6 \mathrm{E} \\
3.4 \mathrm{E} \\
3.0 \mathrm{E}\end{array}$ & $\begin{array}{l}0 . \\
9 . \\
7 .\end{array}$ & $\begin{array}{l}+00 \\
-09 \\
-09 \\
-08\end{array}$ & $\begin{array}{l}0.0 \mathrm{E}+00 \\
2.6 \mathrm{E}-04 \\
1.7 \mathrm{E}-04 \\
1.0 \mathrm{E}-03\end{array}$ & $\begin{array}{l}0.0 \mathrm{E}+00 \\
4.6 \mathrm{E}-04 \\
4.1 \mathrm{E}-04 \\
2.5 \mathrm{E}-03\end{array}$ \\
\hline TOTAL & $1.8 E-03$ & $3.5 \mathrm{E}$ & 7. & -08 & $1.4 E-03$ & $3.4 \mathrm{E}-03$ \\
\hline & Dose Commitr & ents (pe & rem) fr & Airborne & e Pathways & \\
\hline & Total Body & GI-LLI & Thyroid & Bone & Liver & Lung \\
\hline $\begin{array}{l}\text { Infant } \\
\text { Child } \\
\text { Teen } \\
\text { Adult }\end{array}$ & $\begin{array}{l}1.1 \mathrm{E}-05 \\
1.4 \mathrm{E}-04 \\
1.0 \mathrm{E}-04 \\
6.2 \mathrm{E}-04\end{array}$ & $\begin{array}{l}1.1 \mathrm{E}-05 \\
1.5 \mathrm{E}-04 \\
1.4 \mathrm{E}-04 \\
8.7 \mathrm{E}-04\end{array}$ & $\begin{array}{l}1.1 \mathrm{E}-05 \\
1.3 \mathrm{E}-04 \\
9.1 \mathrm{E}-05 \\
5.4 \mathrm{E}-04\end{array}$ & $\begin{array}{l}1.4 \mathrm{E}-05 \\
2.1 \mathrm{E}-04 \\
1.5 \mathrm{E}-04 \\
8.2 \mathrm{E}-04\end{array}$ & $\begin{array}{l}1.3 \mathrm{E}-05 \\
1.6 \mathrm{E}-04 \\
1.1 \mathrm{E}-04 \\
6.4 \mathrm{E}-04\end{array}$ & $\begin{array}{l}1.0 \mathrm{E}-04 \\
1.8 \mathrm{E}-03 \\
1.6 \mathrm{E}-03 \\
6.6 \mathrm{E}-03\end{array}$ \\
\hline TOTAL & $8.7 \mathrm{E}-04$ & 1.2E-03 & $7.7 \mathrm{E}-04$ & 1. $2 E-03$ & $9.3 E-04$ & $1.0 E-02$ \\
\hline
\end{tabular}

Production/consumption factors:

Produce: $1.1 \quad$ Milk: $8.0 \quad$ Meat: 2.8

FRACTION OF POPULATION RECEIVING AN INDICATED AVERAGE TOTAL-BODY DOSE COMMITMENT FROM AIRBORNE PATHWAYS

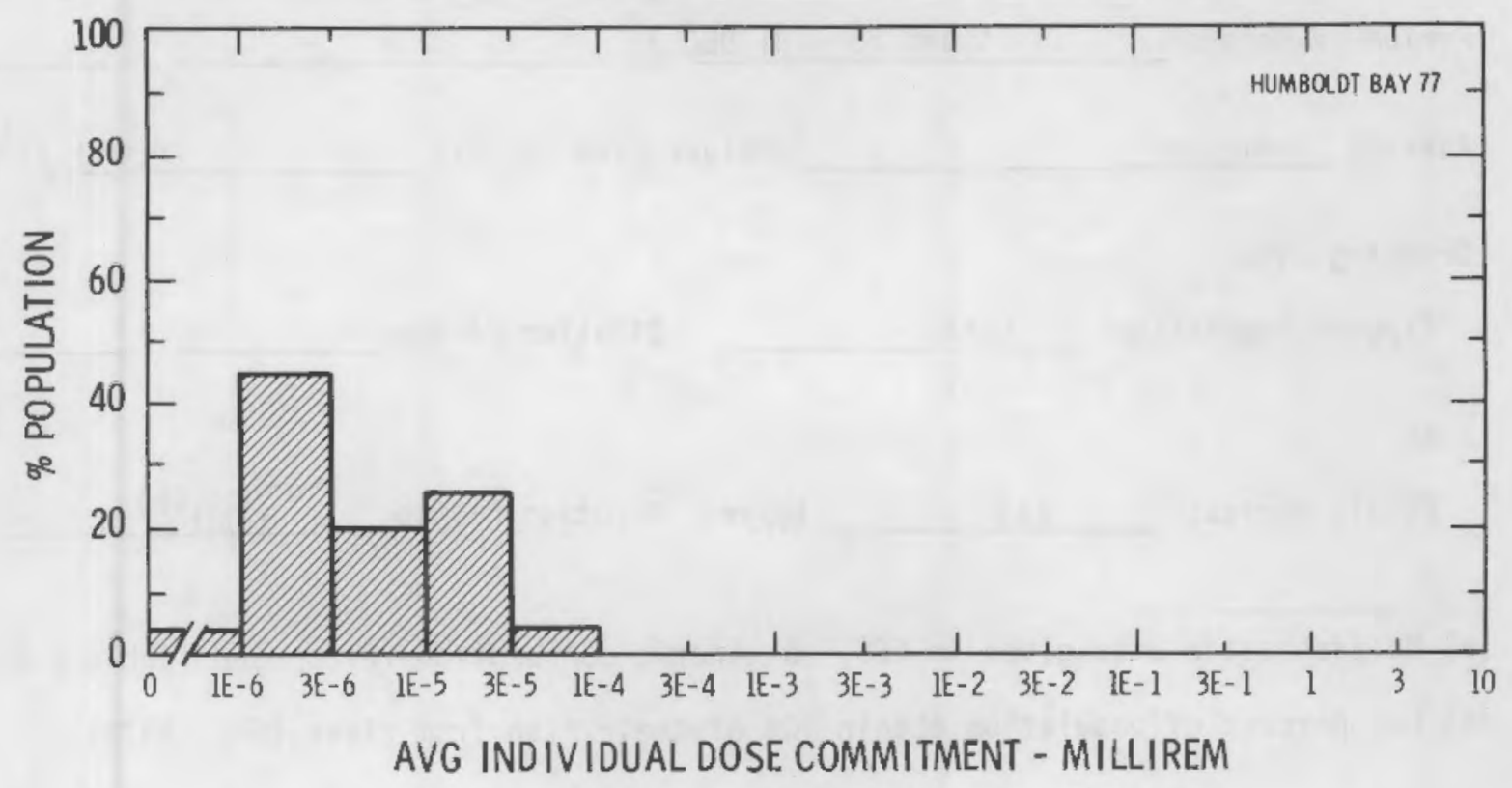


Site INDIAN POINT

Location $\mathrm{N}$ $41 . .2656^{\circ}$

Total Population Within 2-to-80-km Region
BUCHANAN, NEW YORK

W $73.9350^{\circ}$ $1.6 \mathrm{E} 7$

Major Metropolitan Centers Within Region

Center

New York SMSA

Newark SMSA

Stamford

Norwalk

Bridgeport

Poughkeepsie

White Plains
Population

$\begin{array}{r}\frac{9,900,000}{2,100,000} \\ \hline 110,000 \\ \hline 78,000 \\ \hline 160,000 \\ \hline 32,000 \\ \hline 50,000 \\ \hline\end{array}$

\section{Location}

\begin{tabular}{cc}
$60 \mathrm{~km}$ & $\mathrm{~S}$ \\
\hline $64 \mathrm{~km}$ & $\mathrm{SSW}$ \\
\hline $42 \mathrm{~km}$ & $\mathrm{SE}$ \\
\hline $48 \mathrm{~km}$ & $\mathrm{ESE}$ \\
\hline $64 \mathrm{~km}$ & $\mathrm{E}$ \\
\hline $48 \mathrm{~km}$ & $\mathrm{~N}$ \\
\hline $30 \mathrm{~km}$ & $\mathrm{SSE}$ \\
\hline
\end{tabular}

Average Annual State Production of Crops and Animal Products in 80-km Radius Circle

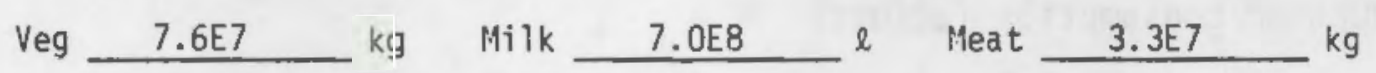

Regional Productivity Factor 0.8

Animal Grazing Factor 0.5

Location of Meteorological Station Site Recovery $96 \%$

Period of Record 1 JAN $75-31$ DEC 75

Average Hudson River Flow at Site $20,000 \mathrm{ft}^{3} / \mathrm{s}$ Drinking Water Exposed Population None 0ilution Factor

Fish

Edible Harvest (a) $\mathrm{kg} / \mathrm{yr}$ Dilution Factor $0.001^{(b)}$

(a) No fish catch data given in FES, so generic consumption rates used (Table A-1). (b) One percent of population obtain 10\% of their fish from river (FES, 1972). 


\section{POPULATION DOSE-COMMITMENT ESTIMATES AND AVERAGE INDIVIDUAL DOSE-COMMITMENT HISTOGRAM FOR INDIAN POINT}

\begin{tabular}{|c|c|c|c|c|c|}
\hline & Total Body & GI-LLI & Thyroid & Bone & Liver \\
\hline $\begin{array}{l}\text { Inf ant } \\
\text { Child } \\
\text { Teen } \\
\text { Adult }\end{array}$ & $\begin{array}{l}0.0 \mathrm{E}+00 \\
3.7 \mathrm{E}-02 \\
7.2 \mathrm{E}-02 \\
7.8 \mathrm{E}-01\end{array}$ & $\begin{array}{l}0.0 \mathrm{E}+00 \\
3.5 \mathrm{E}-03 \\
7.2 \mathrm{E}-03 \\
6.2 \mathrm{E}-02\end{array}$ & $\begin{array}{l}0.0 \mathrm{E}+00 \\
4.2 \mathrm{E}-03 \\
3.0 \mathrm{E}-03 \\
2.0 \mathrm{E}-02\end{array}$ & $\begin{array}{l}0.0 \mathrm{E}+00 \\
1.9 \mathrm{E}-01 \\
1.1 \mathrm{E}-01 \\
6.6 \mathrm{E}-01\end{array}$ & $\begin{array}{l}0.0 \mathrm{E}+00 \\
2.1 \mathrm{E}-01 \\
1.8 \mathrm{E}-01 \\
1.1 \mathrm{E}+00\end{array}$ \\
\hline TOTAL & $8.8 E-01$ & 7.2E-02 & $2.7 \mathrm{E}-02$ & $9.6 \mathrm{E}-01$ & $1.5 \mathrm{E}+00$ \\
\hline
\end{tabular}

Dose Commitments (person-rem) from Airborne Pathways

\begin{tabular}{|c|c|c|c|c|c|c|}
\hline & Total Body & GI-LLI & Thyroid & Bone & Liver & Lung \\
\hline $\begin{array}{l}\text { Inf ant } \\
\text { Child } \\
\text { Teen } \\
\text { Adult }\end{array}$ & $\begin{array}{l}1.7 \mathrm{E}-01 \\
1.9 \mathrm{E}+00 \\
1.4 \mathrm{E}+\mathrm{D0} \\
8.4 \mathrm{E}+00\end{array}$ & $\begin{array}{l}1.7 \mathrm{E}-01 \\
1.9 \mathrm{E}+00 \\
1.4 \mathrm{E}+00 \\
8.4 \mathrm{E}+00\end{array}$ & $\begin{array}{l}2.8 E-01 \\
2.7 E+00 \\
1.8 E+00 \\
1.0 E+01\end{array}$ & $\begin{array}{l}1.7 \mathrm{E}-01 \\
1.9 \mathrm{E}+00 \\
1.4 \mathrm{E}+00 \\
8.3 \mathrm{E}+00\end{array}$ & $\begin{array}{l}1.7 \mathrm{E}-01 \\
1.9 \mathrm{E}+00 \\
1.4 \mathrm{E}+00 \\
8.4 \mathrm{E}+00\end{array}$ & $\begin{array}{l}1.8 \mathrm{E}-01 \\
2.0 \mathrm{E}+00 \\
1.6 \mathrm{E}+00 \\
9.0 \mathrm{E}+00\end{array}$ \\
\hline TOTAL & 1. $2 \mathrm{E}+01$ & $1.2 \mathrm{E}+01$ & $1.5 \mathrm{E}+01$ & $1.2 \mathrm{E}+01$ & 1. $2 \mathrm{E}+01$ & $1.3 \mathrm{E}+01$ \\
\hline
\end{tabular}

Production/consumption factors:

Produce: $<1$ Milk: $<1 \quad$ Meat: <1

FRACTION OF POPULATION RECEIVING AN INDICATED AVERAGE

TOTAL-BODY DOSE COMMITMENT FROM AIRBORNE PATHWAYS

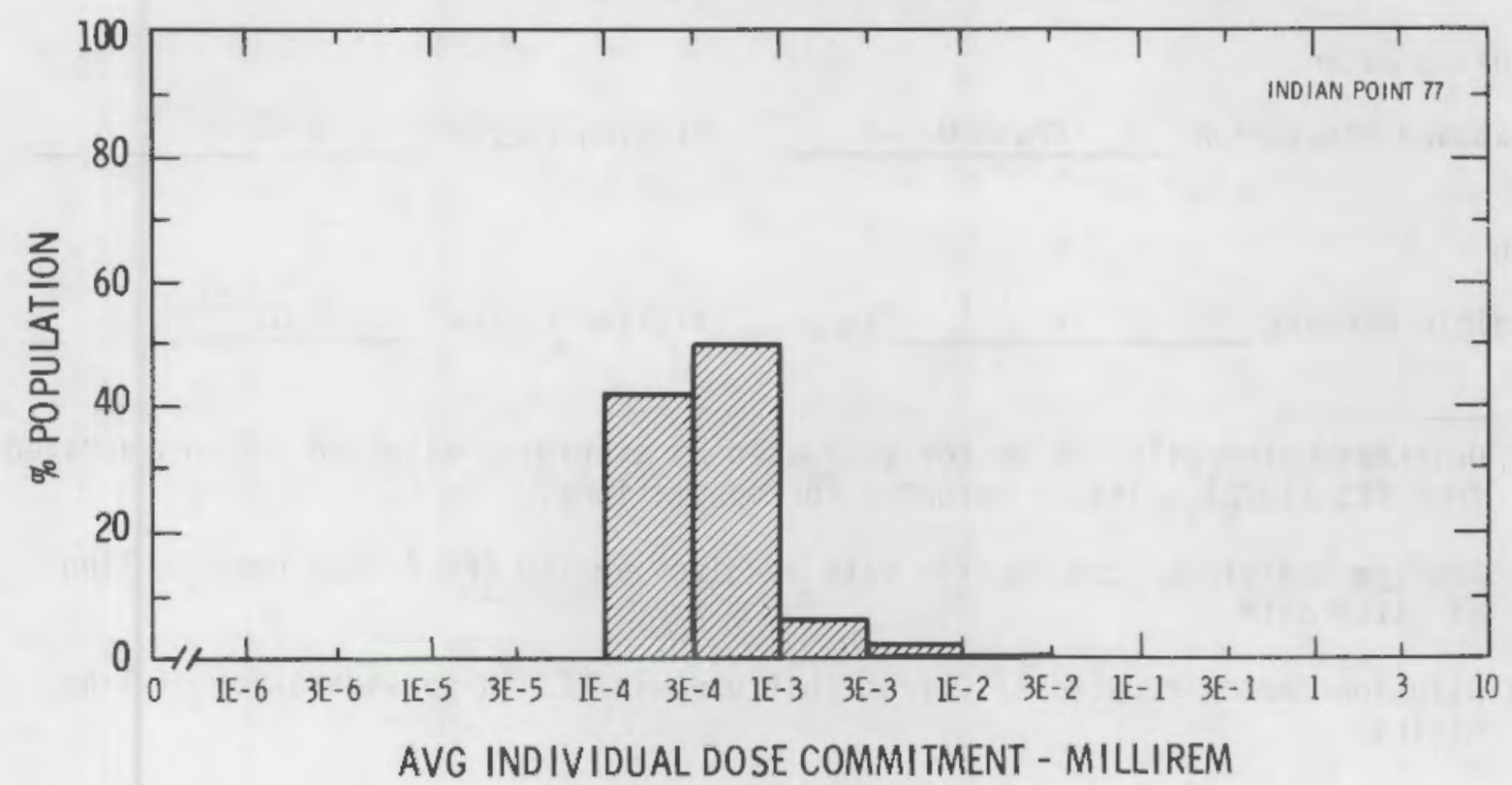


Site KEWAUNEE

Location $N$ $44.3433^{\circ}$

Tota 1 Population Within 2-to-80-km Region
CARLTON, WISCONSIN

W $87.5350^{\circ}$

Major Metropolitan Centers Within Region

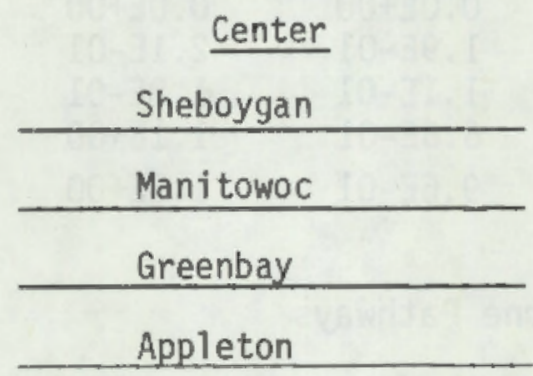

\begin{tabular}{c}
$\frac{\text { Population }}{51,000}$ \\
\hline 35,000 \\
\hline 93,000 \\
\hline 61,000 \\
\hline
\end{tabular}

Location

Average Annual State Production of Crops and Animal Products in 80-km Radius Circle Veg 7.2E7 $\mathrm{kg} \quad \mathrm{MiTk}$ 1.2E9 \& Meat $1.0 \mathrm{E} 8$ $\mathrm{kg}$

Regional Productivity Factor

\begin{tabular}{cc}
$64 \mathrm{~km}$ & $\mathrm{SSW}$ \\
\hline $30 \mathrm{~km}$ & $\mathrm{SSW}$ \\
\hline $40 \mathrm{~km}$ & $\mathrm{~W}$ \\
\hline $72 \mathrm{~km}$ & WSW \\
\hline
\end{tabular}

Animal Grazing Factor 0.5 Location of Meteorological Station Site 0.5 Period of Record 2 JAN $69-31$ DEC 69

Average Water Dilution Flow from Plant $140 \quad \mathrm{ft}^{3} / \mathrm{s}$ Drinking Water Exposed Population 190,000 Dilution Factor $8.2 \mathrm{E}-3^{(\mathrm{a})}$

Fish

Edible Harvest $1.1^{(b)}$ $\mathrm{kg} / \mathrm{yr}$ Dilution Factor $0.01^{(c)}$

(a) Drinking water dilution factor estimated by averaging dilution factors derived from FES (1972) suitably weighted for populations.

(b) Average individual consumption rate as given in the FES (1972) used in lieu of catch data.

(c) Dilution factor reduced $1 / 10$ from that used in FES in consideration of lake mixing. 


\section{POPULATION DOSE-COMMITMENT ESTIMATES ANO AVERAGE INDIVIOUAL DOSE-COMMITMENT HISTOGRAM FOR KEWAUNEE}

Oose Commitments (person-rem) from Liquid Pathways

Infant
Child
Teen
Adult
TOTAL

Inf ant
Child
Teen
Adult
TOTAL

Production/consumption factors:

Produce: $<1$

$\frac{\text { Thyroid }}{2.4 \mathrm{E}-02}$

$1.9 \mathrm{E}-01$

$6.3 \mathrm{E}-02$

4.5E-01

7.3E-01

4. $0 \mathrm{E}-01$

4. $9 \mathrm{E}-01$

7.3E-01

Dose Commitments (person-rem) from Airborne Pathways

\begin{tabular}{|c|c|c|c|c|c|}
\hline Total Body & GI-LLI & Thyroid & Bone & Liver & Lung \\
\hline $\begin{array}{l}3.0 \mathrm{E}-04 \\
3.5 \mathrm{E}-03 \\
2.5 \mathrm{E}-03 \\
1.5 \mathrm{E}-02\end{array}$ & $\begin{array}{l}2.9 \mathrm{E}-04 \\
3.3 \mathrm{E}-03 \\
2.4 \mathrm{E}-03 \\
1.4 \mathrm{E}-02\end{array}$ & $\begin{array}{l}3.3 \mathrm{E}-03 \\
2.6 \mathrm{E}-02 \\
1.2 \mathrm{E}-02 \\
5.4 \mathrm{E}-02\end{array}$ & $\begin{array}{l}3.1 \mathrm{E}-04 \\
4.3 \mathrm{E}-03 \\
3.0 \mathrm{E}-03 \\
1.7 \mathrm{E}-02\end{array}$ & $\begin{array}{l}3.0 \mathrm{E}-04 \\
3.4 \mathrm{E}-03 \\
2.4 \mathrm{E}-03 \\
1.4 \mathrm{E}-02\end{array}$ & $\begin{array}{l}3.2 \mathrm{E}-04 \\
3.7 \mathrm{E}-03 \\
2.9 \mathrm{E}-03 \\
1.6 \mathrm{E}-02\end{array}$ \\
\hline 2.1E-02 & 2. $0 \mathrm{E}-02$ & $9.6 \mathrm{E}-02$ & $2.5 E-02$ & $2.0 E-02$ & 2. $3 E-02$ \\
\hline
\end{tabular}

FRACTION OF POPULATION RECEIVING AN INOICATED AVERAGE TOTAL-BODY DOSE COMMITMENT FROM AIRBORNE PATHWAYS

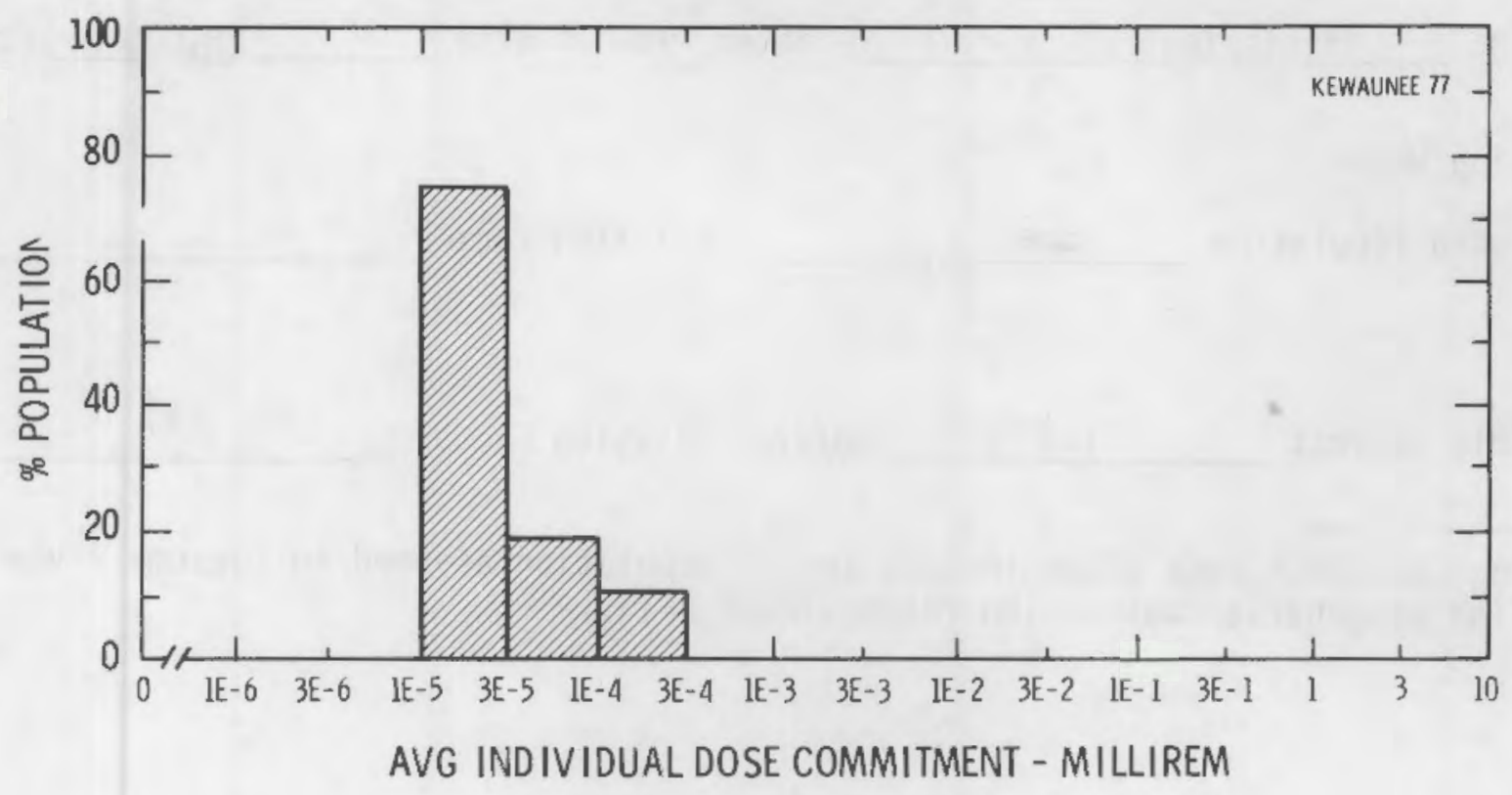


Site LA CROSSE

Location $\mathrm{N}$ $43.5597^{\circ}$

Tota 1 Population Within 2-to-80-km Region
GENOA, WISCONSIN

$91.2281^{\circ}$

Major Metropolitan Centers Within Region

Center
La Crosse

$\begin{array}{r}\frac{\text { Population }}{54,000} \\ \hline\end{array}$

Location

La Crosse

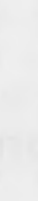

Average Annual State Production of Crops and Animal Products in $80-\mathrm{km}$ Radius Circle

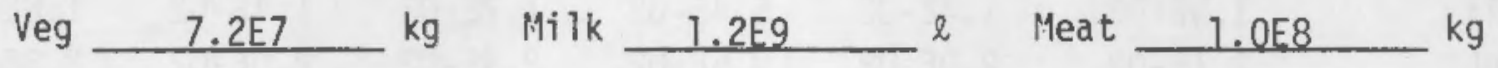

Regional Productivity Factor

Animal Grazing Factor $32 \mathrm{~km}$ N

Location of Meteorological Station Site Recovery $\quad 97 \%$

Period of Record 1 JAN $75-31$ DEC 75

Average Mississippi River Flow at Site $28,000 \quad \mathrm{ft}^{3} / \mathrm{s}$ Drinking Water Exposed Population None Dilution Factor

Fish

Edible Harvest (a) kg/yr Dilution Factor $0.5^{(a)}$

(a) No fish catch data given in FES, so $1 / 2$ population assumed to consume river fish at generic consumption rates (Table $\mathrm{A}-1$ ). 


\section{POPULATION OOSE-COMMITMENT ESTIMATES ANO AVERAGE INDIVIOUAL OOSE-COMMITMENT HISTOGRAM FOR LA CROSSE}

Inf ant

Child

Teen

Adult

TOTAL

Dose Commitments (person-rem) from Liquid Pathways

\begin{tabular}{|c|c|c|c|c|}
\hline Total Body & GI-LLI & Thyroid & Bone & Liver \\
\hline $\begin{array}{l}0.0 \mathrm{E}+00 \\
3.6 \mathrm{E}-01 \\
6.4 \mathrm{E}-01 \\
6.8 \mathrm{E}+00\end{array}$ & $\begin{array}{l}0.0 \mathrm{E}+00 \\
3.4 \mathrm{E}-02 \\
7.3 \mathrm{E}-02 \\
6.4 \mathrm{E}-01\end{array}$ & $\begin{array}{l}0.0 \mathrm{E}+00 \\
3.2 \mathrm{E}-02 \\
2.3 \mathrm{E}-02 \\
1.5 \mathrm{E}-01\end{array}$ & $\begin{array}{l}0.0 \mathrm{E}+00 \\
1.6 \mathrm{E}+00 \\
1.0 \mathrm{E}+00 \\
5.9 \mathrm{E}+00\end{array}$ & $\begin{array}{l}0.0 \mathrm{E}+00 \\
1.8 \mathrm{E}+00 \\
1.6 \mathrm{E}+00 \\
9.3 \mathrm{E}+00\end{array}$ \\
\hline $7.8 \mathrm{E}+00$ & 7. $4 E-01$ & 2.1E-01 & $8.5 E+00$ & 1. $3 \mathrm{E}+01$ \\
\hline
\end{tabular}

Dose Commitments (person-rem) from Airborne Pathways

Infant

Child

Teen

Adult

TOTAL

\section{GI-LL}

2.2E-02

2. $6 \mathrm{E}-01$

2.0E-01

1. $2 \mathrm{E}+00$

$1.1 E+00$

$1.6 \mathrm{E}+00$

$1.6 \mathrm{E}+00$

$\begin{array}{ll}\frac{\text { Thyroid }}{2.7 \mathrm{E}-01} & \frac{\text { Bone }}{2.3 \mathrm{E}-02} \\ 3.4 \mathrm{E}+00 & 2.8 \mathrm{E}-01 \\ 1.9 \mathrm{E}+00 & 1.9 \mathrm{E}-01 \\ 8.6 \mathrm{E}+00 & 1.1 \mathrm{E}+00 \\ 1.4 \mathrm{E}+01 & 1.6 \mathrm{E}+00\end{array}$

\begin{tabular}{|c|c|}
\hline Liver & Lung \\
\hline $\begin{array}{l}2.4 \mathrm{E}-02 \\
2.8 \mathrm{E}-01 \\
2.0 \mathrm{E}-01 \\
1.2 \mathrm{E}+00\end{array}$ & $\begin{array}{l}2.2 \mathrm{E}-02 \\
2.5 \mathrm{E}-01 \\
1.9 \mathrm{E}-01 \\
1.1 \mathrm{E}+00\end{array}$ \\
\hline $1.7 E+00$ & $1.6 \mathrm{E}+00$ \\
\hline
\end{tabular}

Production/consumption factors:

Produce: 1.1

Milk: 27

Meat: 3.8

FRACTION OF POPULATION RECEIVING AN INDICATED AVERAGE TOTAL-BODY OOSE COMMITMENT FROM AIRBORNE PATHWAYS

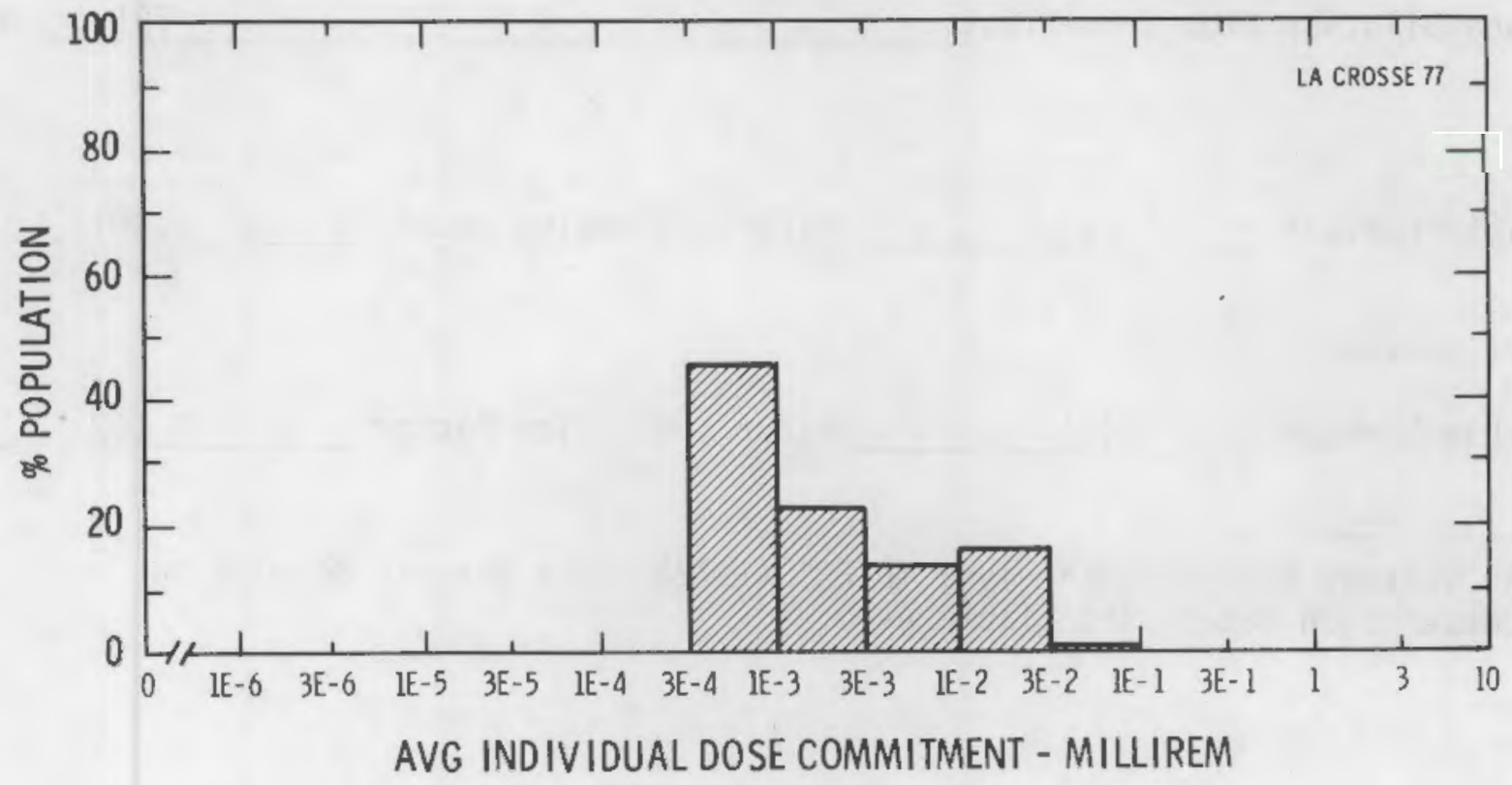


Site

Location $\mathrm{N}$

$43.9503^{\circ}$

W

$69.6964^{\circ}$

Total Population Within 2-to-80-km Region

Major Metropolitan Centers Within Region

$\frac{\begin{array}{c}\text { Center } \\ \text { Portland }\end{array}}{\text { Lewiston }}$

$\frac{\frac{\text { Population }}{71,000}}{46,000}$

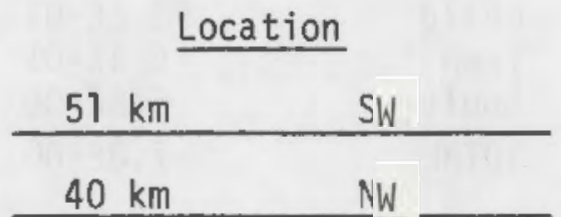

Average Annual State Production of Crops and Animal Products in 80-km Radius Circle Veg 2. $4 \mathrm{E} 8$ $\mathrm{kg} \quad \mathrm{Mi} \mathrm{lk}$ $6.6 \mathrm{E} 7$ \& Meat 4. $3 \mathrm{E} 6$ $\mathrm{kg}$

Regional Productivity Factor

Site $\quad \frac{0.6}{\frac{0.5}{\frac{0.5}{9}}}$

Location of Meteorological Station Site

Period of Record 1 APR 75 - 31 MAR 76

Average Dilution Flow from Plant 791 $f t^{3} / s$

Fish

Edible Harvest (a) $\mathrm{kg} / \mathrm{yr}$ Dilution Factor 0.001

Invertebrates

Edible Harvest (a) $\mathrm{kg} / \mathrm{yr}$ Dilution Factor 0.002

(a) No seafood harvest data given in FES (1972), thus generic population consumption rates used (Table $\mathrm{A}-1$ ). 


\section{POPULATION DOSE-COMMITMENT ESTIMATES AND AVERAGE INDIVIDUAL DOSE-COMMITMENT HISTOGRAM FOR MAINE YANKEE}

Inf ant

Child

Teen

Adult

TOTAL
Infant

Child

Teen

Adult

TOTAL

Dose Commitments (person-rem) from Liquid Pathways

\begin{tabular}{|c|c|c|c|c|}
\hline Total Body & GI-LLI & Thyroid & Bone & Liver \\
\hline $\begin{array}{l}0.0 \mathrm{E}+00 \\
2.7 \mathrm{E}-04 \\
3.7 \mathrm{E}-04 \\
3.6 \mathrm{E}-03\end{array}$ & $\begin{array}{l}0.0 E+00 \\
2.5 E-04 \\
5.1 E-04 \\
4.6 E-03\end{array}$ & $\begin{array}{l}0.0 \mathrm{E}+00 \\
1.8 \mathrm{E}-03 \\
1.3 \mathrm{E}-03 \\
8.5 \mathrm{E}-03\end{array}$ & $\begin{array}{l}0.0 \mathrm{E}+00 \\
8.2 \mathrm{E}-04 \\
4.8 \mathrm{E}-04 \\
2.8 \mathrm{E}-03\end{array}$ & $\begin{array}{l}0.0 \mathrm{E}+00 \\
9.2 \mathrm{E}-04 \\
7.8 \mathrm{E}-04 \\
4.6 \mathrm{E}-03\end{array}$ \\
\hline $4.2 \mathrm{E}-03$ & $5.4 \mathrm{E}-03$ & 1.2E-02 & 4.1E-03 & $6.3 \mathrm{E}-03$ \\
\hline
\end{tabular}

Dose Commitments (person-rem) from Airborne Pathways

\begin{tabular}{|c|c|c|c|c|c|}
\hline Total Body & GI-LLI & Thyroid & Bone & Liver & Lung \\
\hline $\begin{array}{l}1.3 \mathrm{E}-04 \\
1.6 \mathrm{E}-03 \\
1.2 \mathrm{E}-03 \\
7.0 \mathrm{E}-03\end{array}$ & $\begin{array}{l}1.3 \mathrm{E}-04 \\
1.6 \mathrm{E}-03 \\
1.1 \mathrm{E}-03 \\
6.8 \mathrm{E}-03\end{array}$ & $\begin{array}{l}9.1 \mathrm{E}-04 \\
1.3 \mathrm{E}-02 \\
5.9 \mathrm{E}-03 \\
2.6 \mathrm{E}-02\end{array}$ & $\begin{array}{l}1.3 \mathrm{E}-04 \\
1.6 \mathrm{E}-03 \\
1.1 \mathrm{E}-03 \\
6.4 \mathrm{E}-03\end{array}$ & $\begin{array}{l}1.4 \mathrm{E}-04 \\
1.8 \mathrm{E}-03 \\
1.2 \mathrm{E}-03 \\
7.1 \mathrm{E}-03\end{array}$ & $7.5 \mathrm{E}-0$ \\
\hline $1.0 \mathrm{E}-02$ & $9.7 \mathrm{E}-03$ & $4.6 \mathrm{E}-02$ & $9.2 E-03$ & $1.0 \mathrm{E}-02$ & $1.1 \mathrm{E}-0$ \\
\hline
\end{tabular}

Production/consumption factors:

Produce: 1.3

Milk: $<1$

Meat: $<1$

FRACTIDN OF POPULATION RECEIVING AN INDICATED AVERAGE TOTAL-8ODY DOSE COMMITMENT FRDM AIRBORNE PATHWAYS

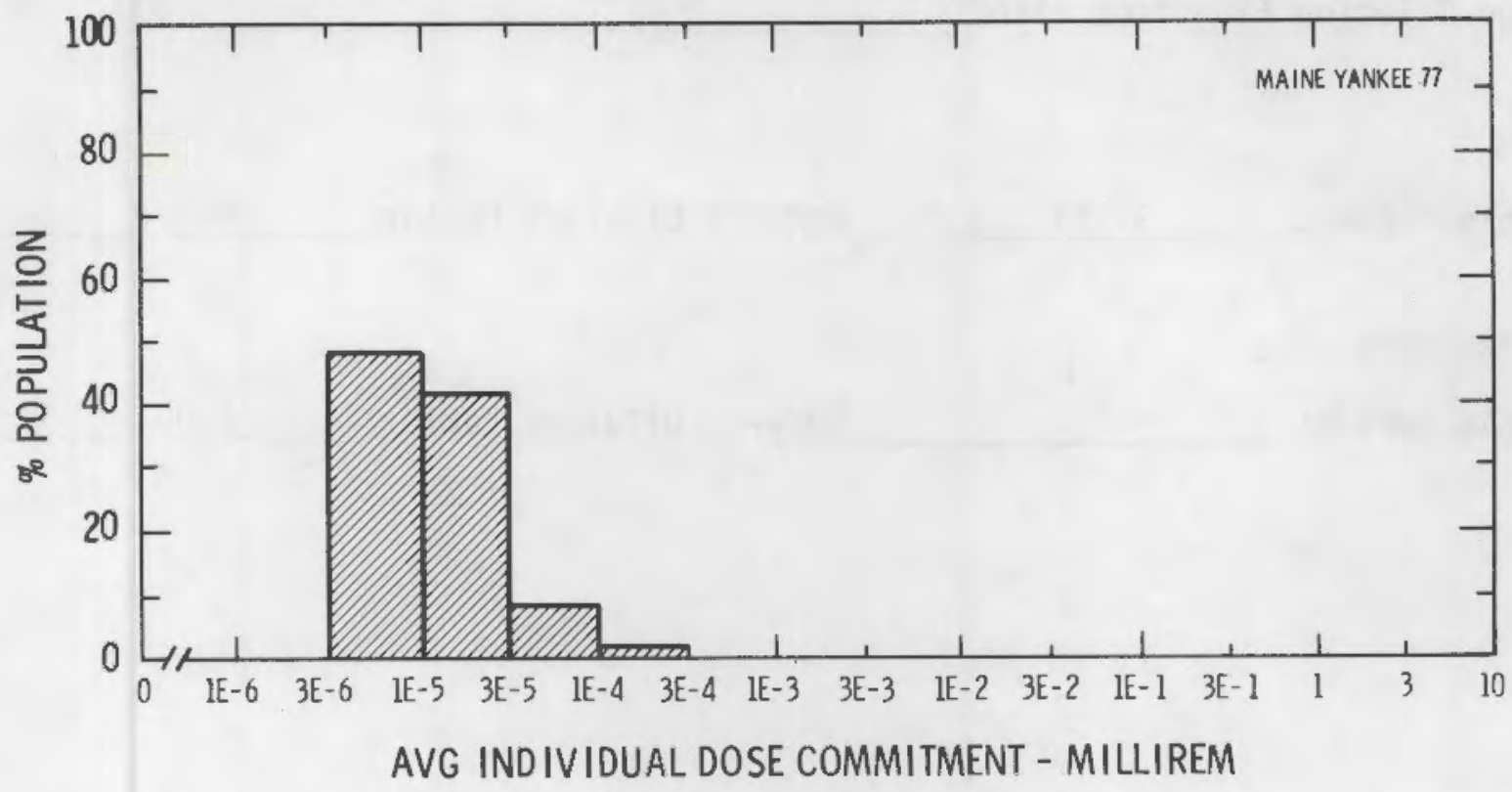


Site MILLSTONE POINT

Location $\mathrm{N}$ $41.3086^{\circ}$

Total Population Within 2-to-80-km Region
WATERFORD, CONNECTICUT

W $72.1675^{\circ}$

Major Metropolitan Centers Within Region

\begin{tabular}{l}
$\frac{1}{\text { Center }}$ \\
Hartford SMSA \\
\hline New Haven SMSA \\
\hline Warwick \\
\hline Waterbury \\
\hline
\end{tabular}

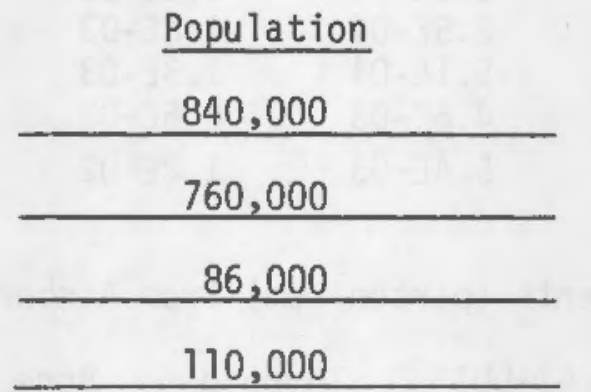

\begin{tabular}{ll}
\multicolumn{2}{r}{ Location } \\
$72 \mathrm{~km}$ & $\mathrm{NW}$ \\
\hline $64 \mathrm{~km}$ & $\mathrm{~W}$ \\
\hline $80 \mathrm{~km}$ & $\mathrm{NE}$ \\
\hline $70 \mathrm{~km}$ & WNW \\
\hline
\end{tabular}

Average Annual State Production of Crops and Animal Products in 80-km Radius Circle

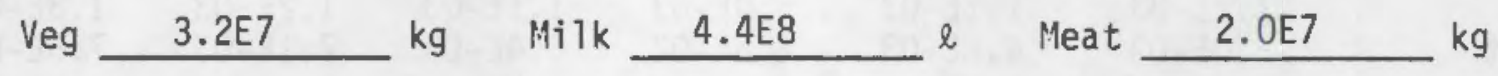

Regional Productivity Factor

Animal Grazing Factor

Location of Meteorological Station Site Recovery $95 \%$

Period of Record 1 JAN 74 - 31 DEC 74

Average Dilution Flow from Plant $300 \mathrm{ft}^{3} / \mathrm{s}$

Fish

Edible Harvest 9. TE4 $\mathrm{kg} / \mathrm{yr}$ Dilution Factor 0.001

Invertebrates

Edible Harvest $9.1 \mathrm{E} 4$ $\mathrm{kg} / \mathrm{yr}$ Dilution Factor 0.002 


\begin{abstract}
POPULATION DOSE-COMMITMENT ESTIMATES AND AVERAGE INDIVIDUAL DOSE-COMMITMENT HISTOGRAM FOR MILLSTONE POINT $1 \& 2$
\end{abstract}

Infant
Child
Teen
Aduit
TOTAL

Dose Commitments (person-rem) from Liquid Pathways

\begin{tabular}{|c|c|c|c|c|}
\hline Total Body & GI-LLI & Thyroid & Bone & Liver \\
\hline $\begin{array}{l}0.0 E+00 \\
3.5 E-03 \\
2.6 E-03 \\
1.8 E-02\end{array}$ & $\begin{array}{l}0.0 \mathrm{E}+00 \\
9.0 \mathrm{E}-03 \\
1.9 \mathrm{E}-02 \\
1.6 \mathrm{E}-01\end{array}$ & $\begin{array}{l}0.0 \mathrm{E}+00 \\
5.3 \mathrm{E}-04 \\
3.7 \mathrm{E}-04 \\
2.5 \mathrm{E}-03\end{array}$ & $\begin{array}{l}0.0 \mathrm{E}+00 \\
1.7 \mathrm{E}-03 \\
1.0 \mathrm{E}-03 \\
6.1 \mathrm{E}-03\end{array}$ & $\begin{array}{l}0.0 \mathrm{E}+00 \\
3.2 \mathrm{E}-03 \\
2.7 \mathrm{E}-03 \\
1.7 \mathrm{E}-02\end{array}$ \\
\hline $2.4 \mathrm{E}-02$ & $1.9 E-01$ & $3.4 \mathrm{E}-03$ & $8.8 E-03$ & $2.3 \mathrm{E}-02$ \\
\hline
\end{tabular}

Dose Commitments (person-rem) from Airborne Pathways

\begin{tabular}{|c|c|c|c|c|c|c|}
\hline & Total Body & GI-LLI & Thyroid & Bone & Liver & Lung \\
\hline $\begin{array}{l}\text { Infant } \\
\text { Child } \\
\text { Teen } \\
\text { Adult }\end{array}$ & $\begin{array}{l}3.2 E+00 \\
3.6 E+01 \\
2.6 E+01 \\
1.6 E+02\end{array}$ & $\begin{array}{l}3.2 \mathrm{E}+00 \\
3.6 \mathrm{E}+01 \\
2.6 \mathrm{E}+01 \\
1.6 \mathrm{E}+02\end{array}$ & $\begin{array}{l}9.8 \mathrm{E}+00 \\
7.1 \mathrm{E}+01 \\
4.1 \mathrm{E}+01 \\
2.1 \mathrm{E}+02\end{array}$ & $\begin{array}{l}3.2 \mathrm{E}+00 \\
3.6 \mathrm{E}+01 \\
2.6 \mathrm{E}+01 \\
1.6 \mathrm{E}+02\end{array}$ & $\begin{array}{l}3.2 \mathrm{E}+00 \\
3.6 \mathrm{E}+01 \\
2.6 \mathrm{E}+01 \\
1.6 \mathrm{E}+02\end{array}$ & $\begin{array}{l}3.3 \mathrm{E}+00 \\
3.7 \mathrm{E}+01 \\
2.7 \mathrm{E}+01 \\
1.6 \mathrm{E}+02\end{array}$ \\
\hline OTAL & $2.2 E+02$ & $2.2 E+02$ & $3.3 E+02$ & $2.2 E+02$ & $2.2 \mathrm{E}+02$ & 2. $3 E+02$ \\
\hline
\end{tabular}

Production/consumption factors:

Produce: $<1 \quad$ Milk: <1 Meat: <1

FRACTION OF POPULATION RECEIVING AN INDICATED AVERAGE

TOTAL-BODY DOSE COMMITMENT FROM AIRBORNE PATHWAYS

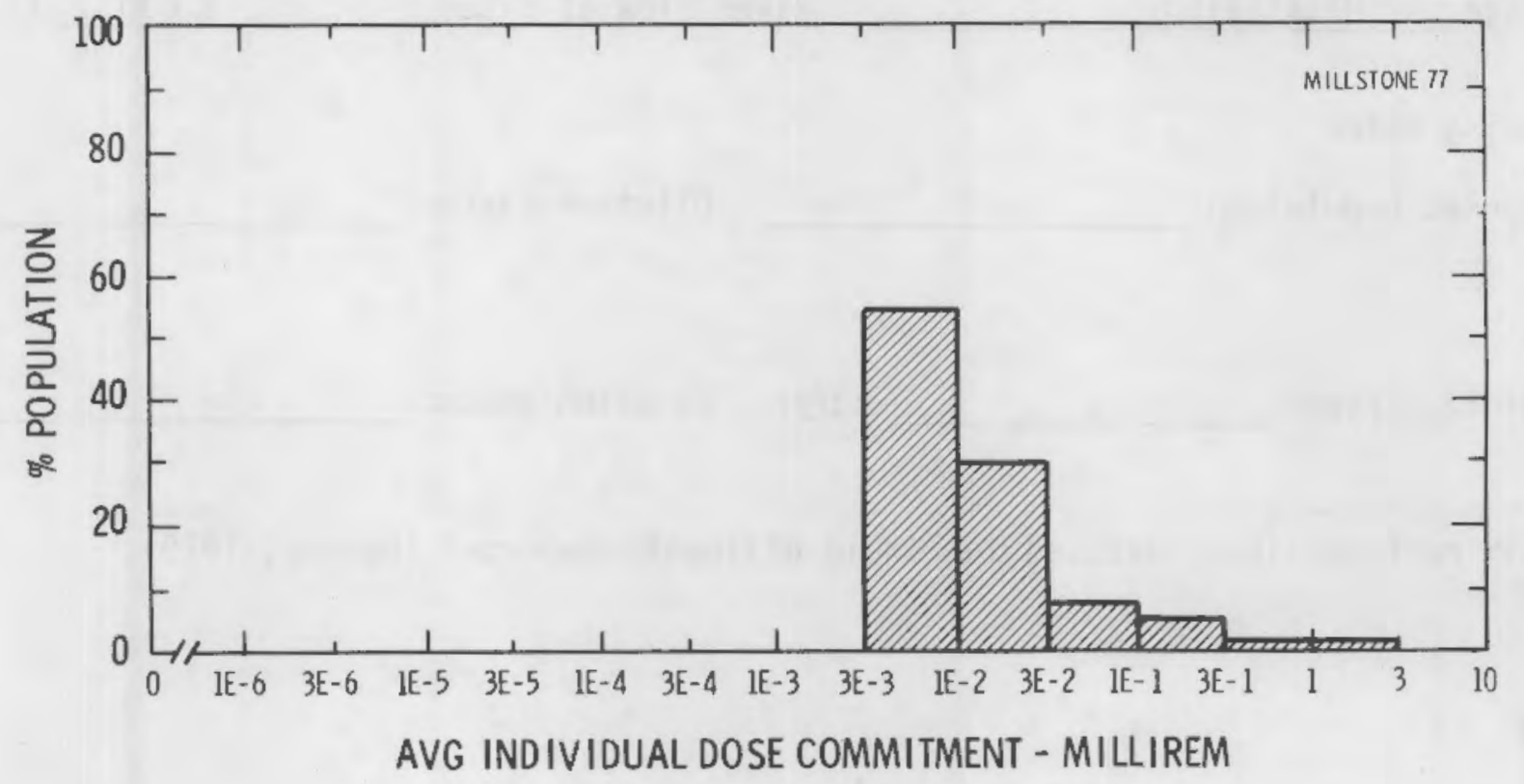


Site

MONTICELLO

Location $\mathrm{N}$

$45.3339^{\circ}$

Total Population Within 2-to-80-km Region
MONTICELLO, MINNESOTA

$93.8486^{\circ}$

$2.1 E 6$

Major Metropolitan Centers Within Region

\section{Center}

Minneapolis-St. Paut SMSA

St. Cloud

Anoka
Population

$2,000,000$

41,000

14.000

\section{Location}

\begin{tabular}{rr}
$64 \mathrm{~km}$ & SE \\
\hline $37 \mathrm{~km}$ & $\mathrm{NW}$ \\
\hline $35 \mathrm{~km}$ & ESE \\
\hline
\end{tabular}

Average Annual State Production of Crops and Animal Products in $80-\mathrm{km}$ Radius Circle

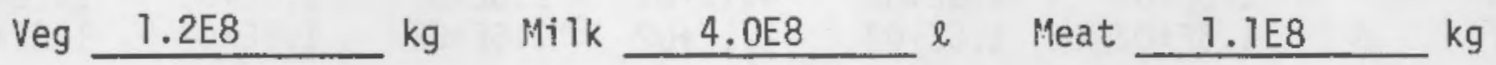

Regional Productivity Factor

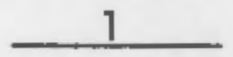

Animal Grazing Factor 0.5

Location of Meteorological Station Site Recovery $92 \%$

Period of Record 1 JAN $74-31$ DEC 74

Average Mississippi

River Flow at Site $4,600 \quad \mathrm{ft}^{3} / \mathrm{s}$

Drinking Water

Exposed Population

(a)

Dilution Factor

Fish

Edible Harvest (a) $\mathrm{kg} / \mathrm{yr}$ Dilution Factor

(a) No radionuclides released in Tiquid effluents reported (Decker, 1979). 


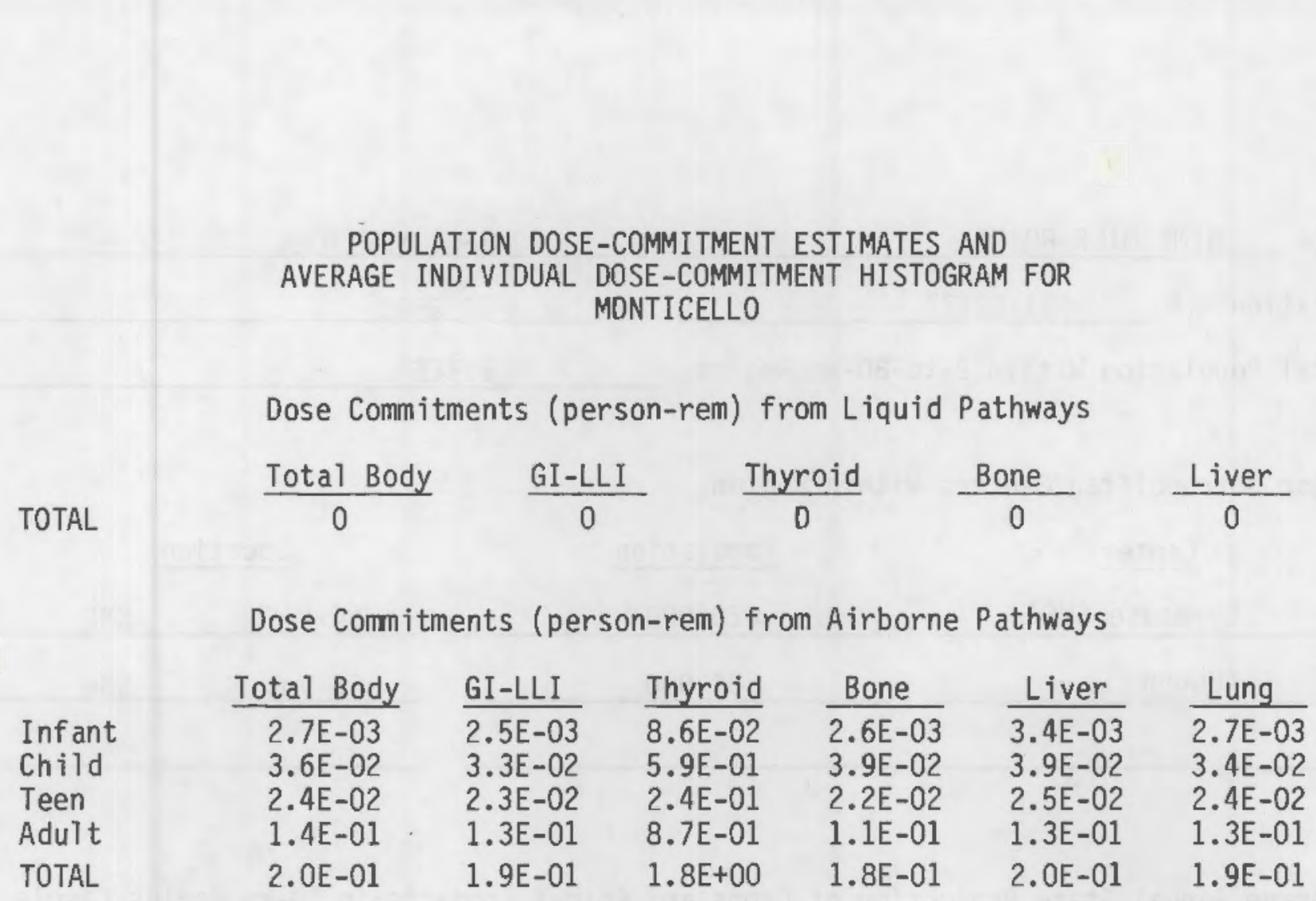

Production/consumption factors:

Produce: <1 Milk: 1.5 Meat: <1

FRACTION OF POPULATION RECEIVING AN INDICATED AVERAGE

TOTAL-BODY DOSE COMMITMENT FROM AIRBORNE PATHWAYS

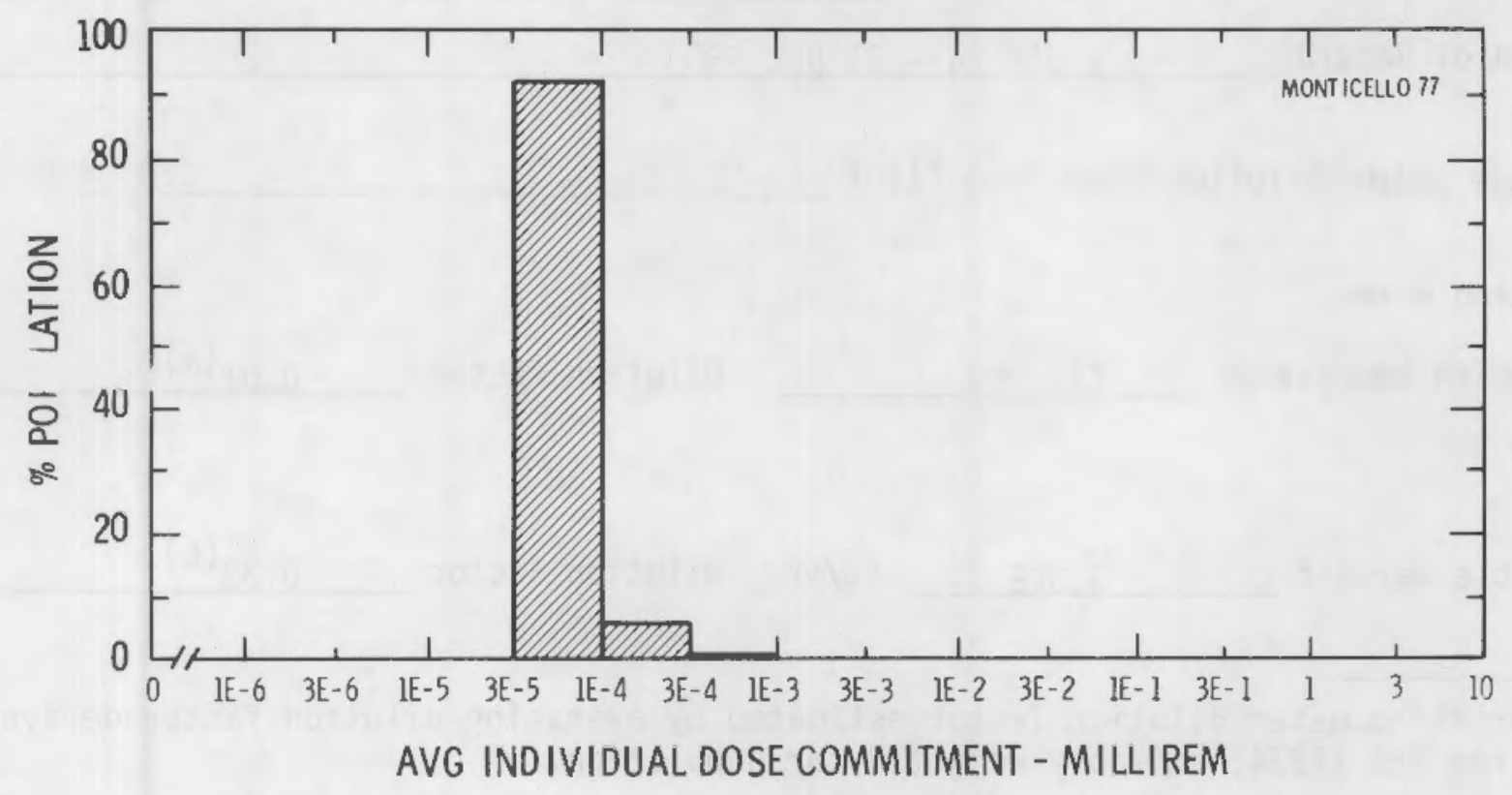


Site NINE MILE POINT

Location N $43.5217^{\circ}$

Total Population Within 2-to-80-km Region
OSWEGO. NEW YORK

W $76.3980^{\circ}$ 8. 3 E.5

Major Metropolitan Centers Within Region

Center

Syracuse SMSA

Auburn
Population

620,000

34,000

Location

$\frac{620,000}{34,000}$

$54 \mathrm{~km}$

$66 \mathrm{~km}$
SSE

SSW

Average Annual State Production of Crops and Animal Products in 80-km Radius Circle

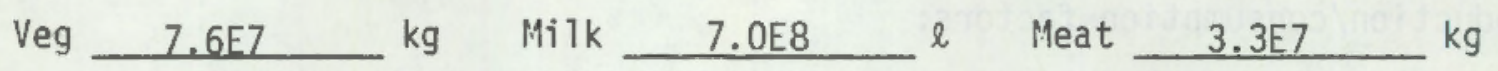

Regional Productivity Factor

0.7

Animal Grazing Factor

0.5

Location of Meteorological Station Site

Recovery $97 \%$

Period of Record

1 JAN 74 - 3] DEC 75

Average Water Dilution Flow from Plant

$270 \quad \mathrm{ft}^{3} / \mathrm{s}$

Drinking Water

Exposed Population

540,000

Dilution Factor

$0.07^{(a)}$

Fish

Edible Harvest

7. 35.5

$\mathrm{kg} / \mathrm{yr}$ Dilution Factor

$0.33^{(b)}$

(a) Drinking water dilution factor estimated by averaging dilution factor derived from FES (1974) suitably weighted for population.

(b) Fish catch dilution factor (FES, 1974). 


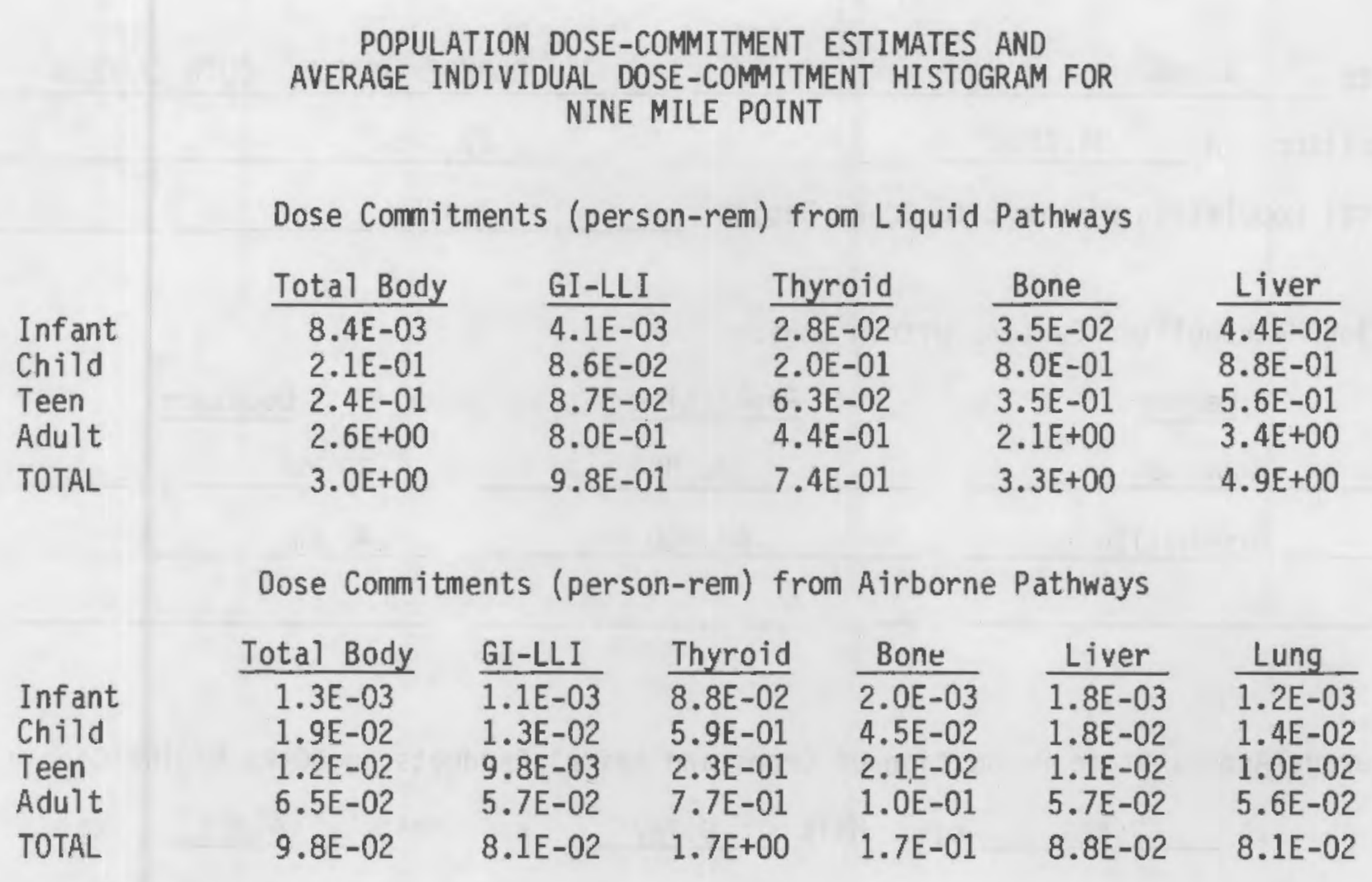

Production/consumption factors:

Produce: <1 Milk: 4.5 Meat: <1

FRACTION OF POPULATION RECEIVING AN INDICATED AVERAGE

TOTAL-BODY DOSE COMMITMENT FROM AIRBORNE PATHWAYS

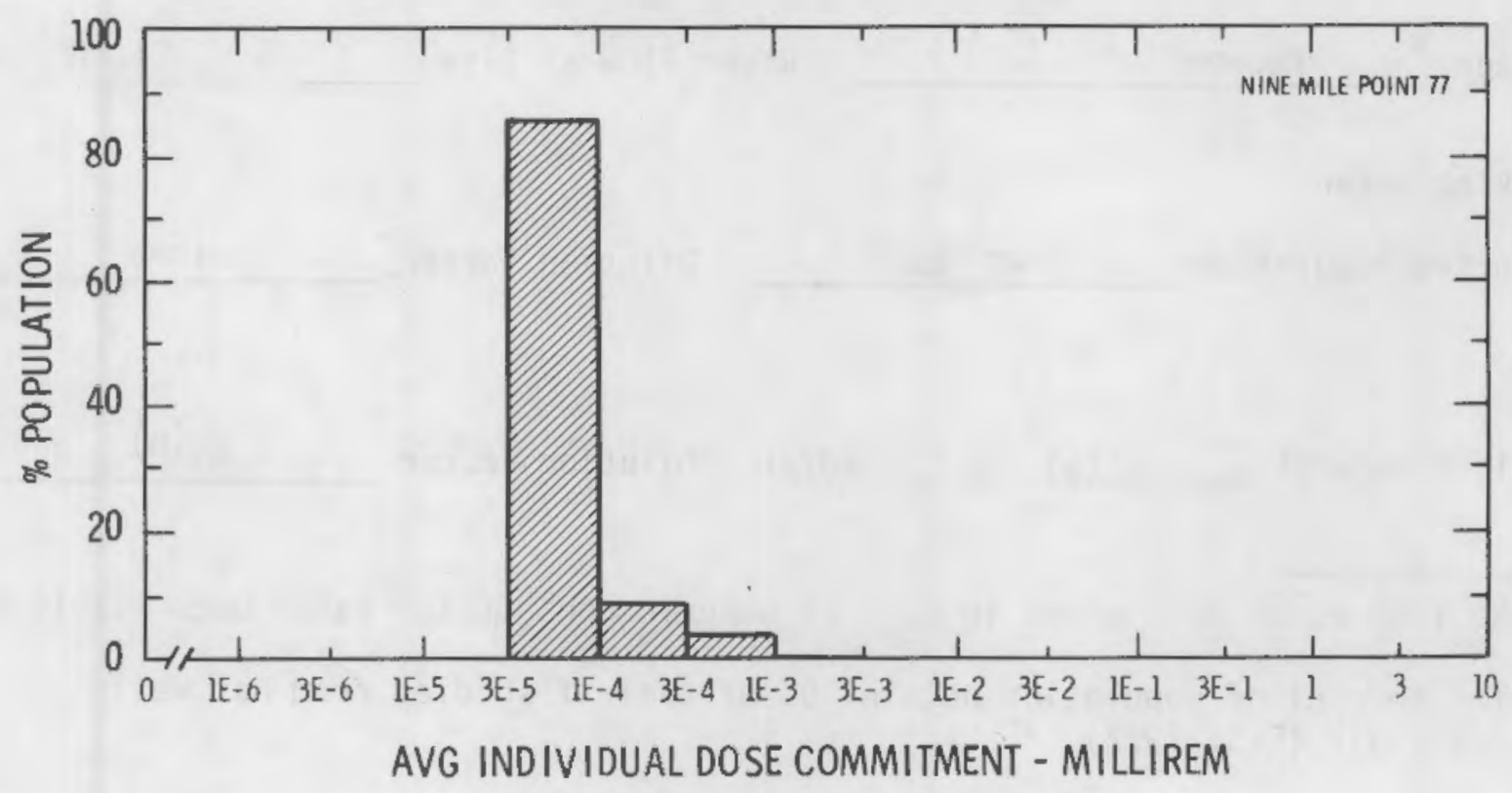


Site OCONEE

OCONEE COUNTY, SOUTH CAROLINA

Location $\mathrm{N} \quad 34.7500^{\circ}$

Total Population Within 2-to-80-km Region

W $\quad 83.0458^{\circ}$

7.4E5

Major Metropolitan Centers Within Region

\begin{tabular}{c}
$\frac{\text { Center }}{\text { Anderson }}$ \\
\hline Greenville \\
\hline
\end{tabular}

Average Annual State Production of Crops and Animal Products in 80-km Radius Circle

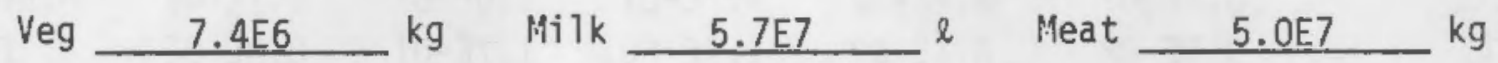

Regional Productivity Factor

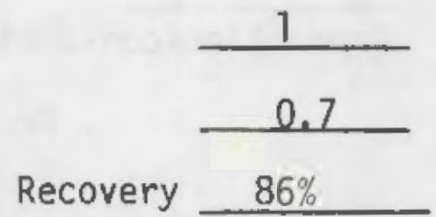

Location of Meteorological Station Site

Recovery $86 \%$

Period of Record

1. JAN $75-31$ DEC 75

Average

Keowee

River Flow at Site

$1,100 \mathrm{ft}^{3} / \mathrm{s}$

Drinking Water

Exposed Population

44,000

Dilution Factor

1

Fish

Edible Harvest

(a)

$\mathrm{kg} / \mathrm{yr}$ Dilution Factor

$0.01^{\text {(b) }}$

(a) No fish catch data given in FES, so generic consumption rates used (Table A-1).

(b) Ten percent of population obtain 10\% of their fish diet from Hartwell Reservoir (FES, 1972). 
POPULATION DOSE-COMMITMENT ESTIMATES AND AVERAGE INDIVIDUAL DOSE-COMMITMENT HISTOGRAM FOR DCONEE $1,2 \& 3$

Dose Commitments (person-rem) from Liquid Pathways

Infant

Child

Teen

Adult

TOTAL
Infant

Child

Teen

Adult

TOTAL

$\frac{G I-L L I}{8.1 E-02}$
$1.2 E+00$
$8.8 E-01$
$7.4 E+00$
$9.5 E+00$

$\frac{\text { Thyroid }}{5.4 \mathrm{E}+00}$

$3.9 \mathrm{E}+01$

$1.2 E+01$

$8.5 \mathrm{E}+01$

$1.4 \mathrm{E}+02$ $\frac{\text { Bone }}{5.6 \mathrm{E}-01}$
$1.2 \mathrm{E}+01$
$5.0 \mathrm{E}+00$
$3.1 \mathrm{E}+01$
$4.8 \mathrm{E}+01$ $\frac{\text { Liver }}{3.3 E-01}$

$9.4 \mathrm{E}+00$

$6.5 \mathrm{E}+00$

4. $0 \mathrm{E}+01$

$5.6 \mathrm{E}+01$

Dose Cormitments (person-rem) from Airborne Pathways

\begin{tabular}{|c|c|c|c|c|c|}
\hline Total Body & GI-LLI & Thyroid & Bone & Liver & Lung \\
\hline $\begin{array}{l}9.9 \mathrm{E}-03 \\
1.1 \mathrm{E}-01 \\
8.0 \mathrm{E}-02 \\
4.9 \mathrm{E}-01\end{array}$ & $\begin{array}{l}9.7 \mathrm{E}-03 \\
1.1 \mathrm{E}-01 \\
8.0 \mathrm{E}-02 \\
4.8 \mathrm{E}-01\end{array}$ & $\begin{array}{l}1.0 \mathrm{E}-01 \\
6.6 \mathrm{E}-01 \\
3.1 \mathrm{E}-01 \\
1.3 \mathrm{E}+00\end{array}$ & $\begin{array}{l}1.0 \mathrm{E}-02 \\
1.1 \mathrm{E}-01 \\
8.0 \mathrm{E}-02 \\
4.8 \mathrm{E}-01\end{array}$ & $\begin{array}{l}1.0 \mathrm{E}-02 \\
1.1 \mathrm{E}-01 \\
8.1 \mathrm{E}-02 \\
4.9 \mathrm{E}-01\end{array}$ & $\begin{array}{l}1.1 \mathrm{E}-02 \\
1.2 \mathrm{E}-01 \\
9.4 \mathrm{E}-02 \\
5.3 \mathrm{E}-01\end{array}$ \\
\hline $6.9 \mathrm{E}-01$ & $6.8 \mathrm{E}-01$ & $2.4 E+00$ & $6.8 \mathrm{E}-01$ & $6.9 E-01$ & $7.5 \mathrm{E}-01$ \\
\hline
\end{tabular}

Production/consumption factors:

Produce: $<1$

Milk: $<1$

Meat: $<1$

FRACTIDN OF POPULATION RECEIVING AN INDICATED AVERAGE TOTAL-BODY DOSE COMMITMENT FROM AIRBORNE PATHWAYS

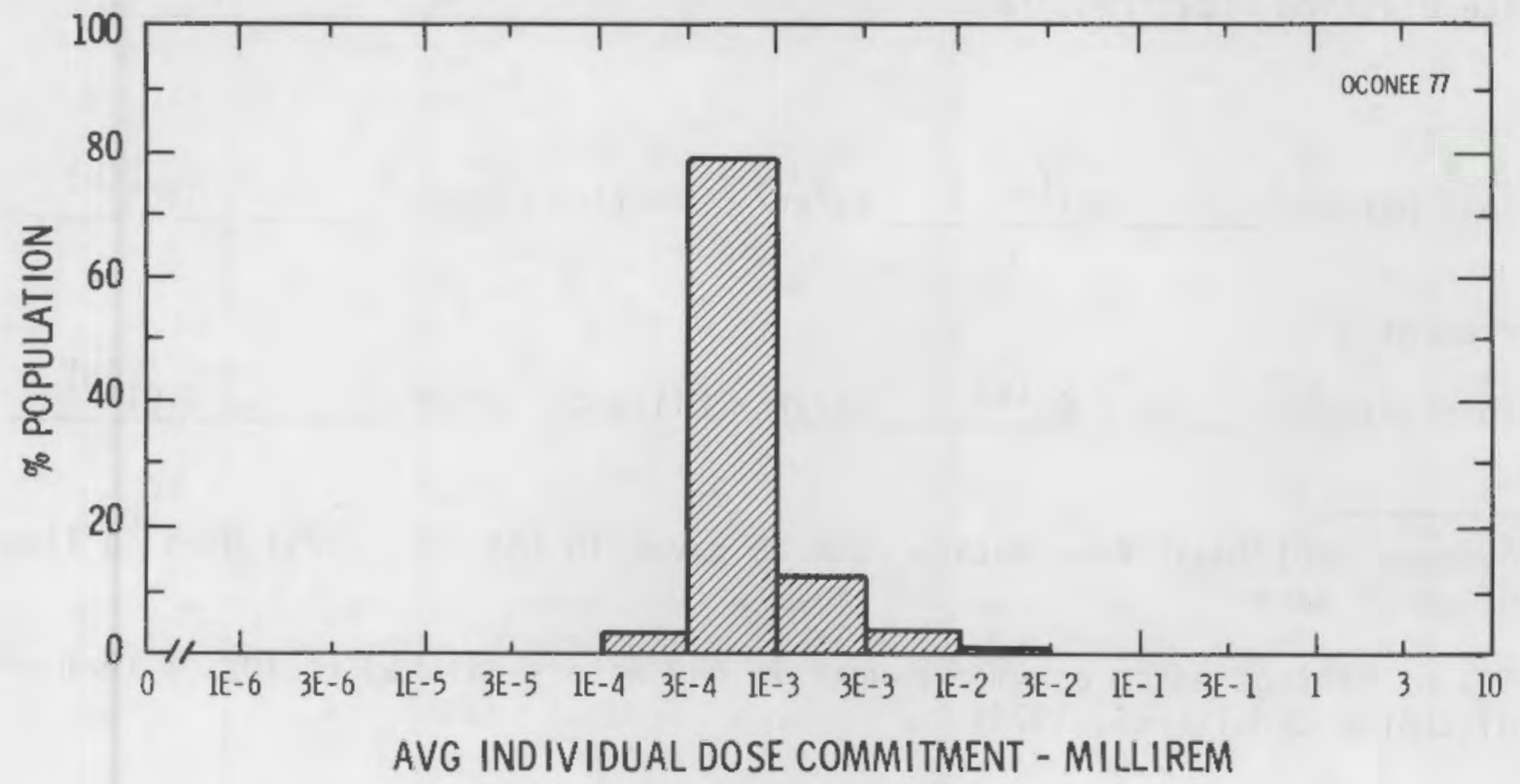


Site OYSTER CREEK

Location $\mathrm{N}$ $39.8739^{\circ}$

Total Population Within 2-to-80-km Region
OYSTER CREEK, NEW JERSEY

W

$3.3 \mathrm{E} 6$

Major Metropolitan Centers Within Region

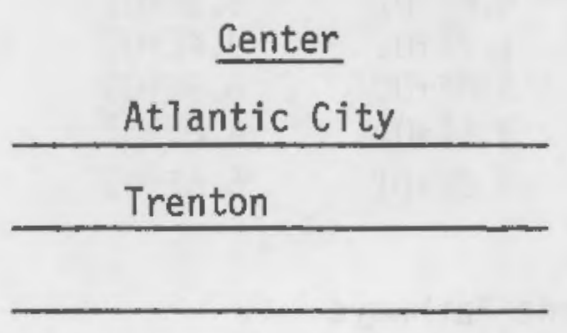

$\frac{\frac{\text { Population }}{49,000}}{110,000}$

\begin{tabular}{|c|c|}
\hline \multicolumn{2}{|c|}{ Location } \\
\hline $56 \mathrm{~km}$ & SSW \\
\hline $64 \mathrm{~km}$ & $\mathrm{NW}$ \\
\hline
\end{tabular}

Average Annual State Production of Crops and Animal Products in $80-\mathrm{km}$ Radius Circle Veg $7.4 E 7$ Mg Milk $2.7 E 8 \quad \ell$ Meat $2.4 E 7$

Regional Productivity Factor 0.5

Animal Grazing Factor 0.6

Location of Meteorological Station

Site

Recovery $63 \%$

Period of Record 15 FEB $66-31$ DEC 68

Average Dilution Flow from Plant $1,800 \mathrm{ft}^{3} / \mathrm{s}$

Fish

Edible Harvest $2.1^{(a)}$ $\mathrm{kg} / \mathrm{yr}$ Dilution Factor $0.01^{(b)}$

Invertebrates

Edible Harvest $0.96^{(a)}$ $\mathrm{kg} / \mathrm{yr}$ Dilution Factor $0.01^{(b)}$

(a) Average individual consumption rate as given in the FES (1974) used in lieu of catch data.

(b) $10 \%$ of seafood eaten assumed caught in bay waters diluted to $10 \%$ of that of discharge canal (FES, 1974). 


\section{POPULATION DOSE-COMMITMENT ESTIMATES AND \\ AVERAGE INOIVIDUAL DOSE-COMMITMENT HISTOGRAM FOR OYSTER CREEK}

Infant
Child
Teen
Adult
TOTAL

Inf ant

Child

Teen

Adult

TOTAL

Dose Commitments (person-rem) from Liquid Pathways

\begin{tabular}{|c|c|c|c|c|}
\hline Total Body & GI-LLI & Thyroid & Bone & Liver \\
\hline $\begin{array}{l}0.0 \mathrm{E}+00 \\
1.8 \mathrm{E}-03 \\
1.3 \mathrm{E}-03 \\
7.9 \mathrm{E}-03\end{array}$ & $\begin{array}{l}0.0 E+00 \\
3.3 E-03 \\
6.9 E-03 \\
6.2 E-02\end{array}$ & $\begin{array}{l}0.0 \mathrm{E}+00 \\
2.8 \mathrm{E}-04 \\
2.0 \mathrm{E}-04 \\
1.3 \mathrm{E}-03\end{array}$ & $\begin{array}{l}0.0 \mathrm{E}+00 \\
7.1 \mathrm{E}-04 \\
5.1 \mathrm{E}-04 \\
3.5 \mathrm{E}-03\end{array}$ & $\begin{array}{l}0.0 \mathrm{E}+00 \\
1.1 \mathrm{E}-03 \\
9.4 \mathrm{E}-04 \\
5.8 \mathrm{E}-03\end{array}$ \\
\hline $1.1 \mathrm{E}-02$ & 7. $2 \mathrm{E}-02$ & $1.8 \mathrm{E}-03$ & 4.7E-03 & $7.8 E-03$ \\
\hline
\end{tabular}

Oose Commitments (person-rem) from Airborne Pathways

Production/consumption factors:

Produce: $<1$

Milk: $<1$

Meat: $<1$

FRACTION OF POPULATION RECEIVING AN INDICATED AVERAGE TOTAL-BODY DOSE COMMITMENT FROM AIRBORNE PATHWAYS

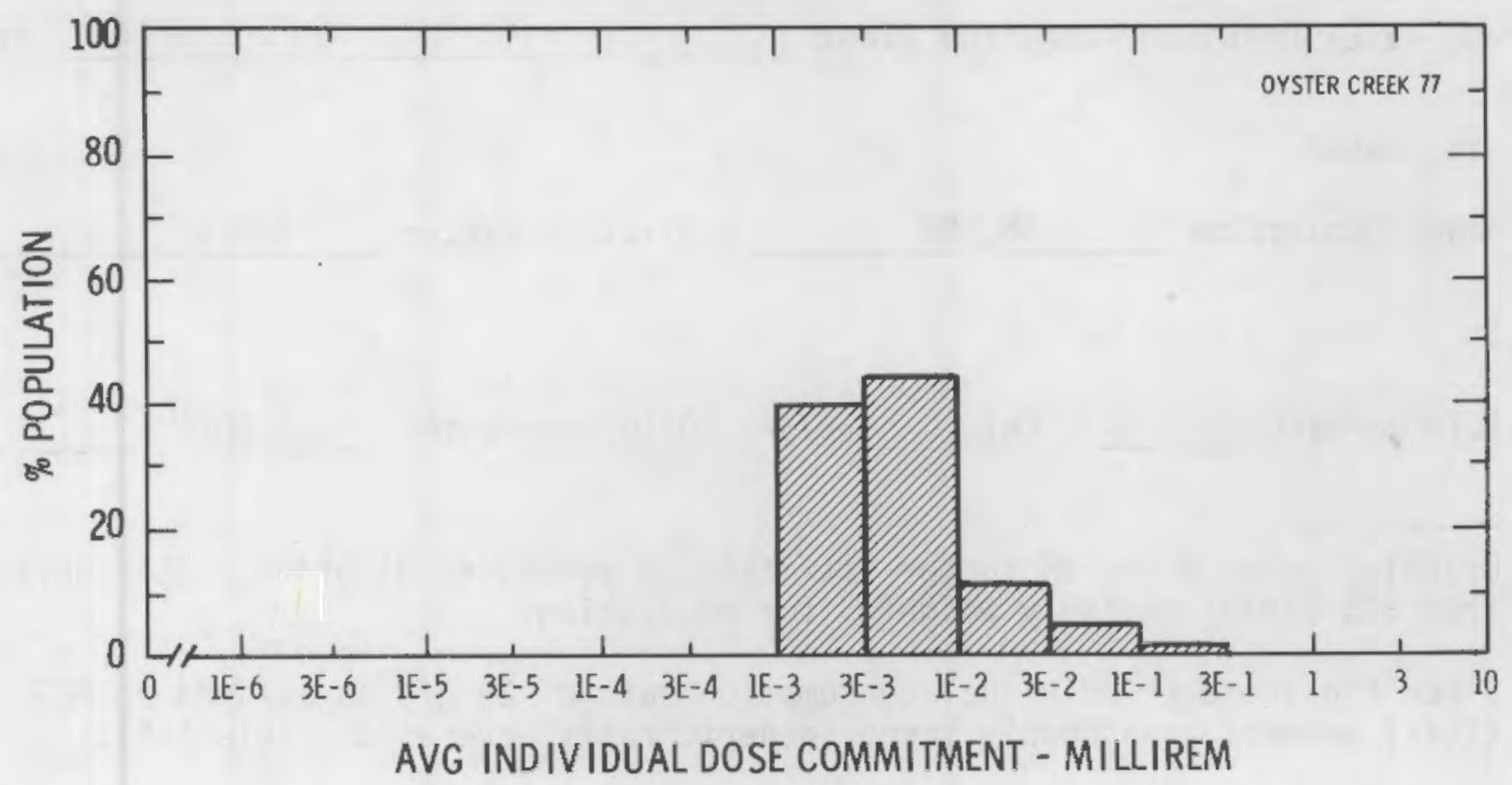


Site PALISADES

COVERT TOWNSHIP, MICHIGAN

Location $\mathrm{N}$

$42.3233^{\circ}$

$86.3142^{\circ}$

Total Population Within 2-to-80-km Region $1.0 \mathrm{E} 6$

Major Metropolitan Centers Within Region

$\frac{\frac{\text { Center }}{\text { Kalamazoo }}}{\text { South Bend SMSA }}$

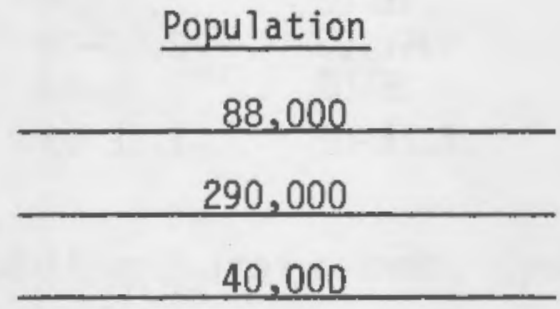

\begin{tabular}{ll}
\multicolumn{2}{c}{ Location } \\
$56 \mathrm{~km}$ & $\mathrm{E}$ \\
\hline $72 \mathrm{~km}$ & $\mathrm{~S}$ \\
\hline $66 \mathrm{~km}$ & $\mathrm{SW}$ \\
\hline
\end{tabular}

Average Annual State Production of Crops and Animal Products in $80-\mathrm{km}$ Radius Circle Veg $6.8 \mathrm{E} 7 \quad k$ $\mathrm{kg} \quad \mathrm{Milk}$ $2.9 E 8$ \& Meat $4.5 \mathrm{E} 7 \mathrm{~kg}$

Regional Productivity Factor Site Recovery $\frac{0.6}{\frac{0.5}{67 \%}}$ Animal Grazing Factor Location of Meteorological Station Site 1 SEP 73 - 31 AUG 74

Period of Record

Average Water Dilution Flow from Plant $140 \mathrm{ft}^{3} / \mathrm{s}$

Drinking Water

Exposed Population 48,000

Dilution Factor $3.5 \mathrm{E}-3^{(a)}$

Fish

Edible Harvest (b) $\mathrm{kg} / \mathrm{yr}$ Dilution Factor $0.007^{(b)}$

(a) Drinking water dilution factor estimated by averaging dilution factor derived from FES (1972) suitably weighted for population.

(b) Since the average individual consumption rate of $20 \mathrm{~g} / \mathrm{d}$ as assumed in FES (1972) seemed unreasonably large, generic rates were used (Table A-1). 


\begin{abstract}
POPULATION DOSE-COMMITMENT ESTIMATES ANO
AVERAGE INDIVIDUAL DOSE-COMMITMENT HISTOGRAM FOR

PALISADES
\end{abstract}

\begin{tabular}{|c|c|c|c|c|c|}
\hline & Total Body & GI-LLI & Thyroid & Bone & Liver \\
\hline $\begin{array}{l}\text { Inf ant } \\
\text { Child } \\
\text { Teen } \\
\text { Adult }\end{array}$ & $\begin{array}{l}8.6 E-05 \\
2.7 E-02 \\
5.1 E-02 \\
5.6 E-01\end{array}$ & $\begin{array}{l}7.3 \mathrm{E}-05 \\
1.9 \mathrm{E}-03 \\
2.4 \mathrm{E}-03 \\
2.0 \mathrm{E}-02\end{array}$ & $\begin{array}{l}7.3 \mathrm{E}-05 \\
8.5 \mathrm{E}-04 \\
3.4 \mathrm{E}-04 \\
2.9 \mathrm{E}-03\end{array}$ & $\begin{array}{l}1.4 \mathrm{E}-04 \\
1.5 \mathrm{E}-01 \\
9.1 \mathrm{E}-02 \\
5.2 \mathrm{E}-01\end{array}$ & $\begin{array}{l}2.5 \mathrm{E}-04 \\
1.6 \mathrm{E}-01 \\
1.3 \mathrm{E}-01 \\
8.0 \mathrm{E}-01\end{array}$ \\
\hline TOTAL & $6.3 \mathrm{E}-01$ & $2.4 \mathrm{E}-02$ & $4.2 E-03$ & $7.7 \mathrm{E}-01$ & $1.1 \mathrm{E}+00$ \\
\hline
\end{tabular}

Dose Commitments (person-rem) from Liquid Pathways

Dose Cormitments (person-rem) from Airborne Pathways

\begin{tabular}{|c|c|c|c|c|c|c|}
\hline & Total Body & GI-LLI & Thyroid & Bone & Liver & Lung \\
\hline $\begin{array}{l}\text { Infant } \\
\text { Child } \\
\text { Teen } \\
\text { Adult }\end{array}$ & $\begin{array}{l}2.6 \mathrm{E}-05 \\
2.9 \mathrm{E}-04 \\
1.9 \mathrm{E}-04 \\
1.0 \mathrm{E}-03\end{array}$ & $\begin{array}{l}1.7 \mathrm{E}-05 \\
2.2 \mathrm{E}-04 \\
1.5 \mathrm{E}-04 \\
8.7 \mathrm{E}-04\end{array}$ & $\begin{array}{l}6.0 \mathrm{E}-03 \\
3.7 \mathrm{E}-02 \\
1.4 \mathrm{E}-02 \\
4.8 \mathrm{E}-02\end{array}$ & $\begin{array}{l}4.3 \mathrm{E}-05 \\
3.8 \mathrm{E}-04 \\
1.7 \mathrm{E}-04 \\
7.6 \mathrm{E}-04\end{array}$ & $\begin{array}{l}5.7 \mathrm{E}-05 \\
4.7 \mathrm{E}-04 \\
2.6 \mathrm{E}-04 \\
1.1 \mathrm{E}-03\end{array}$ & $\begin{array}{l}2.2 \mathrm{E}-05 \\
2.6 \mathrm{E}-04 \\
1.9 \mathrm{E}-04 \\
9.7 \mathrm{E}-04\end{array}$ \\
\hline TOTAL & $1.5 E-03$ & 1. $3 \mathrm{E}-03$ & $1.1 E-01$ & $1.4 \mathrm{E}-03$ & $1.9 \mathrm{E}-03$ & $1.4 \mathrm{E}-03$ \\
\hline
\end{tabular}

Production/consumption factors:

Produce: <1 Milk: 1.3 Meat: <1

FRACTION OF POPULATION RECEIVING AN INDICATED AVERAGE

TOTAL-BODY DOSE COMMITMENT FROM AIRBORNE PATHWAYS

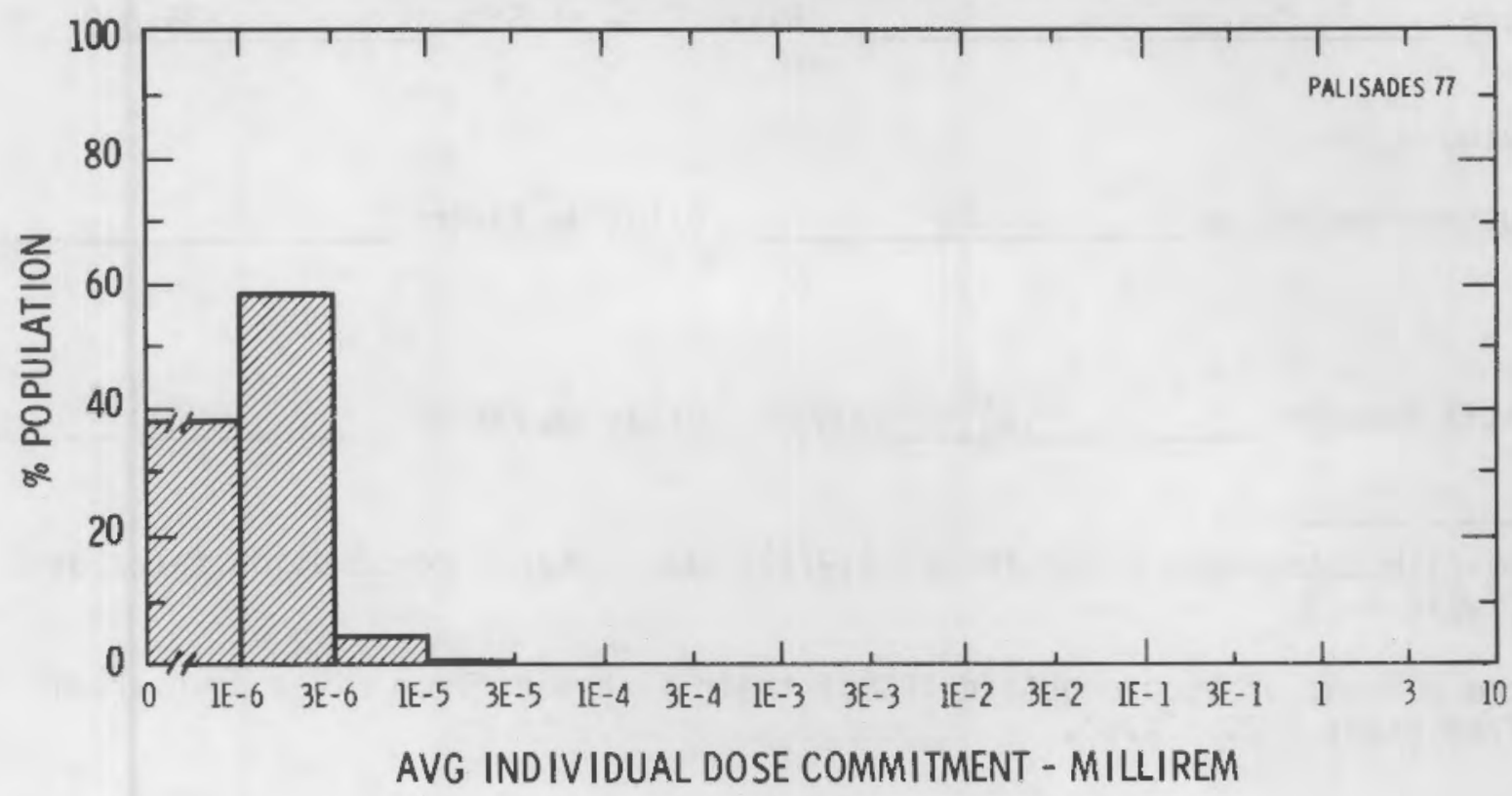


Site PEACH BOTTOM

Location N $39.7592^{\circ}$

Total Population Within 2-to-80-km Region
YORK COUNTY, PENNSYLVANIA

W

$76.2689^{\circ}$

4.1E6

Major Metropolitan Centers Within Region

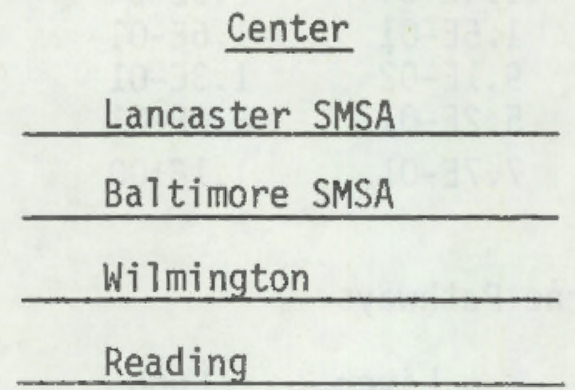

$\begin{array}{r}\frac{\text { Population }}{320,000} \\ \hline 2,100,000 \\ \hline 500,000 \\ \hline 300,000 \\ \hline\end{array}$

\begin{tabular}{cc}
\multicolumn{2}{c}{ Location } \\
$31 \mathrm{~km}$ & $\mathrm{~N}$ \\
\hline $58 \mathrm{~km}$ & $\mathrm{SW}$ \\
\hline $64 \mathrm{~km}$ & $\mathrm{E}$ \\
\hline $72 \mathrm{~km}$ & $\mathrm{NNE}$ \\
\hline
\end{tabular}

Average Annual State Production of Crops and Animal Products in 80-km Radius Circle Veg $5.3 \mathrm{E} 7 \mathrm{~kg} \mathrm{Milk}-5.3 \mathrm{E} 8{ }^{\ell}$ Meat $\frac{5.4 \mathrm{E} 7}{\mathrm{~kg}}$

Regional Productivity Factor

Anima 1 Grazing Factor

Location of Meteorological Station Site 0.6

Period of Record 1 AUG 67 - 31 JUL 71

Average Susquehanna

River Flow at Site $36,000 \mathrm{ft}^{3} / \mathrm{s}$

Drinking Water

Exposed Population 2.1E6

Dilution Factor 1

Fish

Edible Harvest

(a) $\mathrm{kg} / \mathrm{yr}$ Dilution Factor

$0.001^{(b)}$

(a) No fish catch data given in FES (1974), thus generic consumption rates used (Table A-1).

(b) One percent of people obtain 10\% of their fish diet from river downstream from plant (FES, 1973). 


\begin{abstract}
POPULATION DOSE-COMMITMENT ESTIMATES AND AVERAGE INDIVIDUAL DOSE-COMMITMENT HISTOGRAM FOR PEACH BOTTOM 2 \& 3
\end{abstract}

Dose Commitments (person-rem) from Liquid Pathways

Infant

Child

Teen

Adult

TOTAL

Inf ant

Child

Teen

Adult

TOTAL

$\frac{G I-L L I}{2.0 E-02}$
$1.9 E-01$
$1.3 E-01$
$1.1 E+00$
$1.5 E+00$

Thyroid

3. $7 \mathrm{E}+01$

1.1E+01

8. $0 \mathrm{E}+01$

1. $3 \mathrm{E}+02$
$6.8 \mathrm{E}-01$

4. $8 E-01$

$5.2 \mathrm{E}+00$

$6.4 \mathrm{E}+00$ $\frac{\text { Bone }}{2.2 \mathrm{E}-01}$

2. $3 \mathrm{E}+00$

6. $3 E-01$

4. $0 \mathrm{E}+00$

7. $2 \mathrm{E}+00$
Liver

$2.9 E+00$

1. $1 \mathrm{E}+00$

7. $0 E+00$

$1.1 \mathrm{E}+01$

Dose Commitments (person-rem) from Airborne Pathways

\begin{tabular}{|c|c|c|c|c|c|}
\hline Total Body & GI-LLI & Thyroid & Bone & Liver & Lung \\
\hline $\begin{array}{l}7.3 \mathrm{E}-02 \\
8.1 \mathrm{E}-01 \\
5.9 \mathrm{E}-01 \\
3.5 \mathrm{E}+00\end{array}$ & $\begin{array}{l}7.3 \mathrm{E}-02 \\
8.0 \mathrm{E}-01 \\
5.9 \mathrm{E}-01 \\
3.5 \mathrm{E}+00\end{array}$ & $\begin{array}{l}5.4 \mathrm{E}-01 \\
3.6 \mathrm{E}+00 \\
1.8 \mathrm{E}+00 \\
7.9 \mathrm{E}+00\end{array}$ & $\begin{array}{l}\text { 7. } 3 \mathrm{E}-02 \\
8.0 \mathrm{E}-01 \\
5.7 \mathrm{E}-01 \\
3.4 \mathrm{E}+00\end{array}$ & $\begin{array}{l}7.7 \mathrm{E}-02 \\
8.3 \mathrm{E}-01 \\
6.0 \mathrm{E}-01 \\
3.6 \mathrm{E}+00\end{array}$ & $\begin{array}{l}7.8 \mathrm{E}-02 \\
8.9 \mathrm{E}-01 \\
6.9 \mathrm{E}-01 \\
3.9 \mathrm{E}+00\end{array}$ \\
\hline $5.0 \mathrm{E}+00$ & $5.0 E+00$ & $1.4 \mathrm{E}+01$ & $4.9 E+00$ & $5.1 E+00$ & $5.5 E+00$ \\
\hline
\end{tabular}

Production/consumption factors:

Produce: $<1$

Milk: $<1$

Meat: <1

FRACTION OF POPULATION RECEIVING AN INDICATED AVERAGE

TOTAL-BODY DOSE COMMITMENT FROM AIRBORNE PATHWAYS

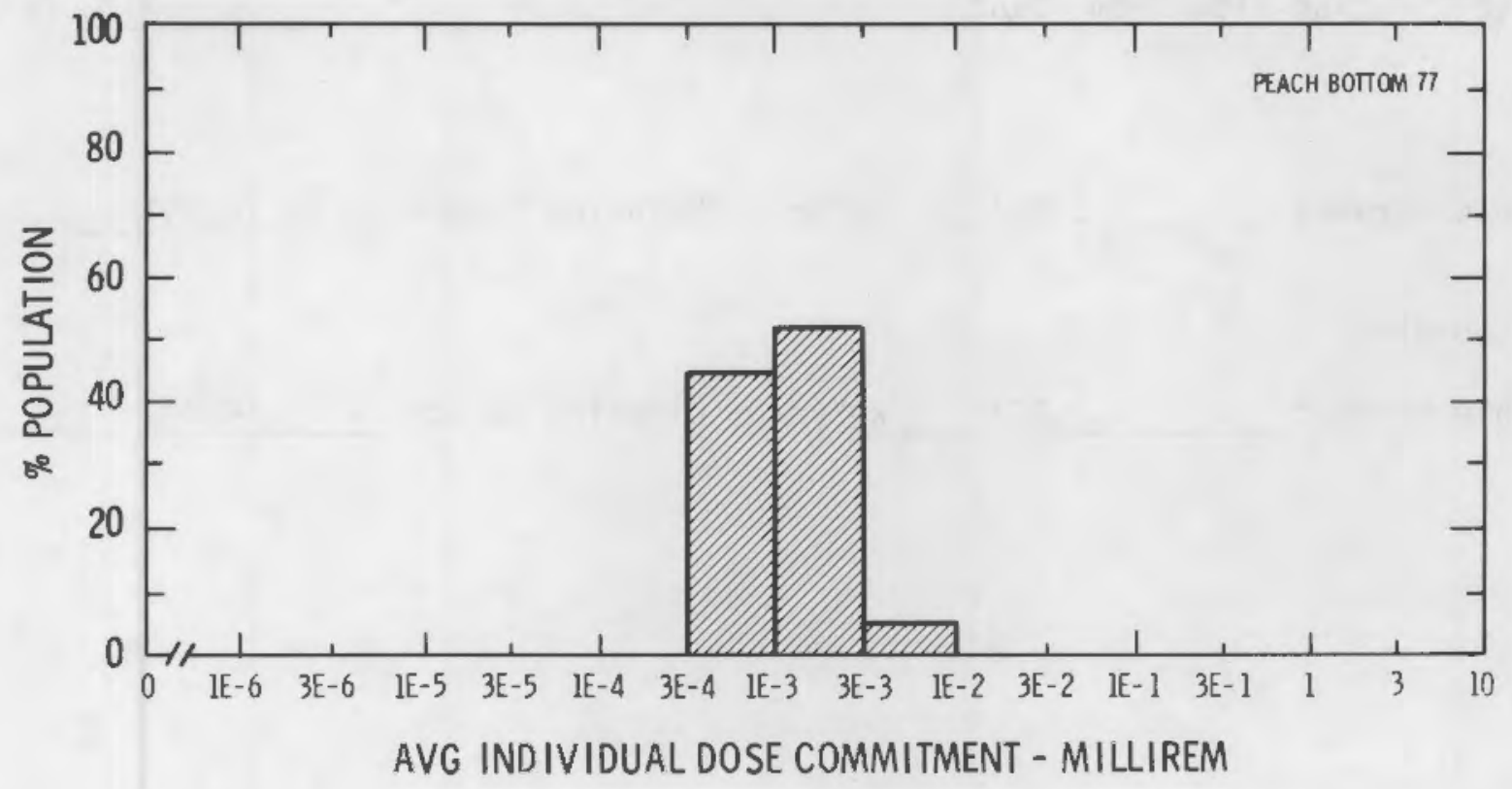


Site PILGRIM

Location $\mathrm{N}$ $41.9444^{\circ}$

Total Population Within 2-to-80-km Region
PLYMOUTH, MASSACHUSETTS

W $70.5778^{\circ}$

Major Metropolitan Centers Within Region

\begin{tabular}{l} 
Boston SMSA \\
\hline Providence SMSA \\
\hline Brockton \\
\hline New Bedford \\
\hline
\end{tabular}

$\begin{array}{r}\frac{\text { Population }}{3,500,000} \\ \hline 780,000 \\ \hline 91,000 \\ \hline 100,000 \\ \hline\end{array}$

\begin{tabular}{ll}
\multicolumn{2}{c}{ Location } \\
$64 \mathrm{~km}$ & $\mathrm{NW}$ \\
\hline $70 \mathrm{~km}$ & WSW \\
\hline $40 \mathrm{~km}$ & WNW \\
\hline $45 \mathrm{~km}$ & $\mathrm{SW}$ \\
\hline
\end{tabular}

Average Annual State Production of Crops and Animal Products in $80-\mathrm{km}$ Radius Circle Veg $2.0 \mathrm{E} 7 \mathrm{~kg}$ Milk $2.6 \mathrm{E} 8$ _ Meat $1.6 \mathrm{E} 7$ kg

Regional Productivity Factor

0.3

Animal Grazing Factor

0.6

Location of Meteorological Station Site Recovery $93 \%$

Period of Record 1 MAY 74 - 30 APR 75

Average Dilution Flow from Plant $27 \mathrm{ft}^{3} / \mathrm{s}$

Fish

Edible Harvest $2.6 \mathrm{E} 4$ $\mathrm{kg} / \mathrm{yr}$ Dilution Factor 0.001

Invertebrates

Edible Harvest 3. $1 E 4$ $\mathrm{kg} / \mathrm{yr}$ Dilution Factor 0.002 
POPULATION DOSE-COMMITMENT ESTIMATES AND

AVERAGE INDIVIDUAL DOSE-COMMITMENT HISTOGRAM FOR

PILGRIM

Dose Commitments (person-rem) from Liquid Pathways

Infant

Child

Teen

Aduit

TOTAL

Infant
Child
Teen
Adult
TOTAL

\begin{tabular}{l} 
Total Body \\
\hline $0.0 \mathrm{E}+00$ \\
$1.7 \mathrm{E}-02$ \\
$1.1 \mathrm{E}-02$ \\
$6.5 \mathrm{E}-02$ \\
$9.3 \mathrm{E}-02$
\end{tabular}

GI-LLI

$0.0 \mathrm{E}+00$

Thyroid

$1.5 \mathrm{E}-02$

$6.8 \mathrm{E}-03$

2. $9 \mathrm{E}-02$

4.7E-03

2.4E-01

3.1E-02

$\frac{\text { Bone }}{0.0 \mathrm{E}+00}$

Liver

$0.0 \mathrm{E}+00$

$4.1 \mathrm{E}-02$

$6.1 \mathrm{E}-02$

$3.2 E-02$

1.9E-01

2.0E-01

2.6E-01

Dose Commitments (person-rem) from Airborne Pathways

\begin{tabular}{|c|c|c|c|c|c|}
\hline Total Body & GI-LLI & Thyroid & Bone & Liver & Lung \\
\hline $\begin{array}{l}7.5 \mathrm{E}-01 \\
8.3 \mathrm{E}+00 \\
6.1 \mathrm{E}+00 \\
3.7 \mathrm{E}+01\end{array}$ & $\begin{array}{l}7.5 \mathrm{E}-01 \\
8.3 \mathrm{E}+00 \\
6.1 \mathrm{E}+00 \\
3.7 \mathrm{E}+01\end{array}$ & $\begin{array}{l}9.6 \mathrm{E}-01 \\
9.6 \mathrm{E}+00 \\
6.7 \mathrm{E}+00 \\
3.9 \mathrm{E}+01\end{array}$ & $\begin{array}{l}7.5 \mathrm{E}-01 \\
8.3 \mathrm{E}+00 \\
6.1 \mathrm{E}+00 \\
3.7 \mathrm{E}+01\end{array}$ & $\begin{array}{l}7.5 E-01 \\
8.3 E+00 \\
6.1 E+00 \\
3.7 E+01\end{array}$ & $\begin{array}{l}7.7 \mathrm{E}-01 \\
8.6 \mathrm{E}+00 \\
6.5 \mathrm{E}+00 \\
3.8 \mathrm{E}+01\end{array}$ \\
\hline $5.2 E+01$ & $5.2 E+01$ & $5.6 E+01$ & $5.2 E+01$ & $5.2 E+01$ & $5.4 E+01$ \\
\hline
\end{tabular}

Production/consumption factors:

Produce: $<1$

Milk: $<1$

Meat: $<1$

FRACTION OF POPULATION RECEIVING AN INDICATED AVERAGE

TOTAL-BODY DOSE COMMITMENT FROM AIRBORNE PATHWAYS

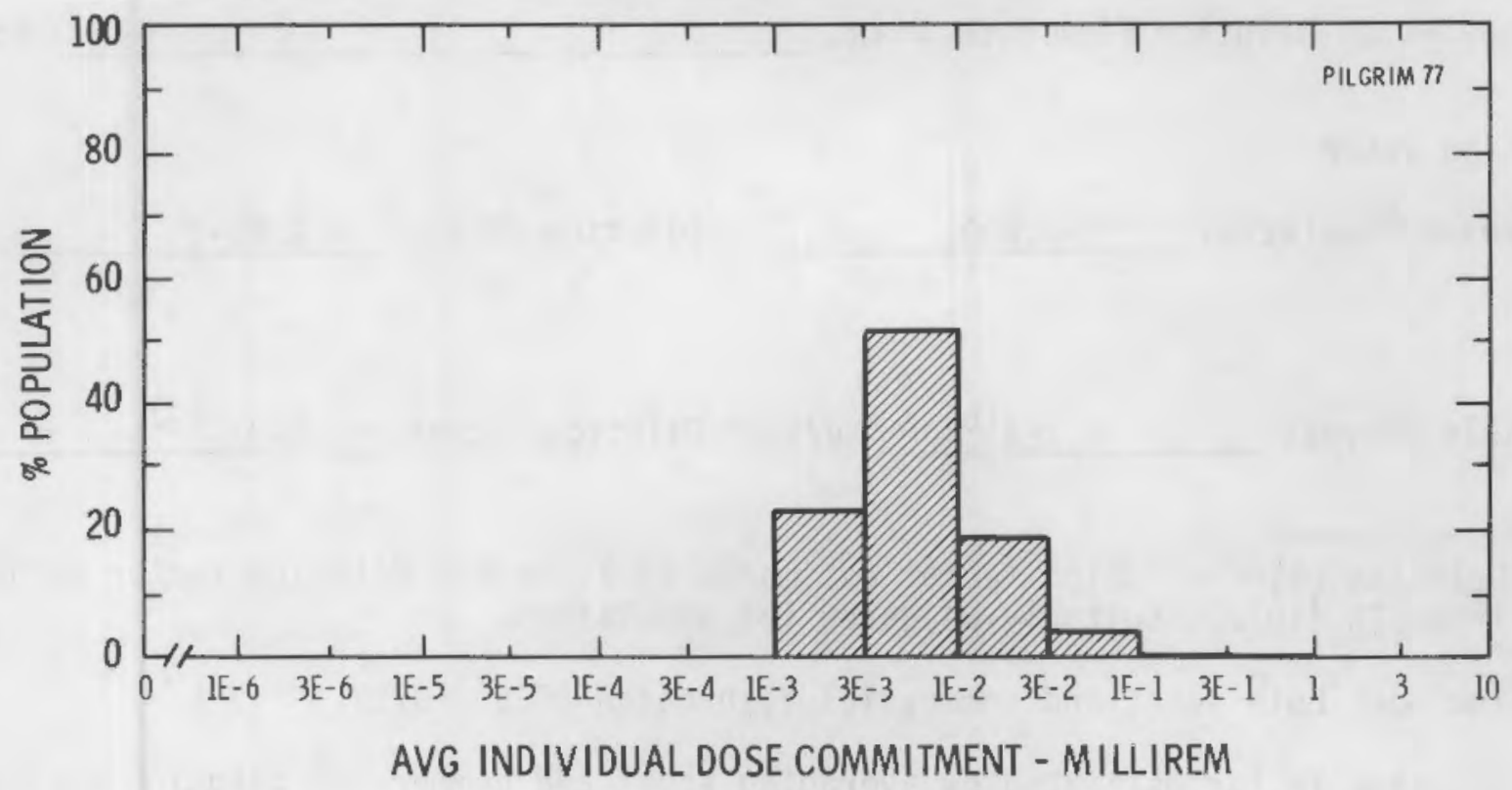


Site POINT BEACH

Location $N$ $44.2764^{\circ}$

Total Population Within 2-to-80-km Region
MANITOWOC COUNTY, WISCONSIN

W $87.5186^{\circ}$

Major Metropolitan Centers Within Region

\begin{tabular}{c}
$\frac{\text { Center }}{\text { Sheboygan }}$ \\
\hline Manitowoc \\
\hline Greenbay \\
\hline Appleton \\
\hline
\end{tabular}

\begin{tabular}{c}
$\frac{\text { Population }}{57,000}$ \\
\hline 35,000 \\
\hline 93,000 \\
\hline 67,000 \\
\hline
\end{tabular}

\begin{tabular}{cc}
\multicolumn{2}{c}{ Location } \\
$56 \mathrm{~km}$ & $\mathrm{SSW}$ \\
\hline $19 \mathrm{~km}$ & $\mathrm{SSW}$ \\
\hline $48 \mathrm{~km}$ & $\mathrm{NW}$ \\
\hline $72 \mathrm{~km}$ & $\mathrm{~W}$ \\
\hline
\end{tabular}

Average Annual State Production of Crops and Animal Products in $80-\mathrm{km}$ Radius Circle

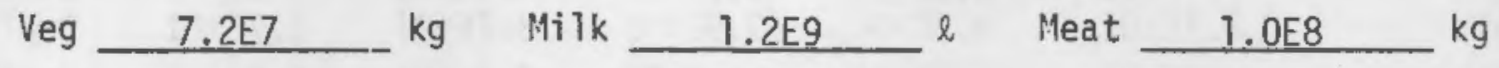

Regional Productivity Factor 0.5

Animal Grazing Factor 0.5

Location of Meteorological Station Site Recovery $83 \%$

Period of Record 19 APR $67-18$ APR 69

Average Water Dilution Flow from Plant $590 \quad \mathrm{ft}^{3} / \mathrm{s}$

Drinking Water

Exposed Population 190,000 Dilution Factor $2.6 \mathrm{E}-3^{(a)}$

Fish

Edible Harvest $6.7 \mathrm{E} 4^{(\mathrm{b})}$ $\mathrm{kg} / \mathrm{yr}$ Dilution Factor $0.013^{(c)}$

(a) Drinking water dilution factor estimated by averaging dilution factor derived from FES (1972), suitably weighted for population.

(b) Includes both sport and commercial fish catch (FES, 1972).

(c) Dilution factor estimated by averaging sport and commercial dilution factor derived from FES (1972). 


\begin{abstract}
POPULATION DOSE-COMMITMENT ESTIMATES AND AVERAGE INDIVIOUAL OOSE-COMMITMENT HISTOGRAM FOR POINT BEACH 1 \& 2
\end{abstract}

\begin{tabular}{|c|c|c|c|c|c|}
\hline & Total Body & GI-LLI & Thyroid & Bone & Liver \\
\hline Inf ant & $7.0 \mathrm{E}-04$ & $6.7 \mathrm{E}-04$ & $\overline{7.0 \mathrm{E}-03}$ & $\overline{2.6 \mathrm{E}-04}$ & $1.0 \mathrm{E}-03$ \\
\hline Child & $1.1 \mathrm{E}-02$ & $7.7 \mathrm{E}-03$ & $5.3 \mathrm{E}-02$ & $1.5 \mathrm{E}-02$ & $2.6 \mathrm{E}-02$ \\
\hline Teen & $8.5 E-03$ & $3.2 \mathrm{E}-03$ & $1.7 \mathrm{E}-02$ & $8.2 E-03$ & $1.7 \mathrm{E}-02$ \\
\hline Adult & $8.6 \mathrm{E}-02$ & $2.8 \mathrm{E}-02$ & 1. $2 \mathrm{E}-01$ & -02 & $1.1 E-01$ \\
\hline TOTAL & 1.1E-01 & $3.9 \mathrm{E}-02$ & $2.0 \mathrm{E}-01$ & 7. $2 \mathrm{E}-02$ & $1.5 E-01$ \\
\hline
\end{tabular}

Dose Commitments (person-rem) from Airborne Pathways

\begin{tabular}{|c|c|c|c|c|c|c|}
\hline & Total Body & GI-LLI & Thyroid & Bone & Liver & Lung \\
\hline $\begin{array}{l}\text { Infant } \\
\text { Child } \\
\text { Teen } \\
\text { Adult }\end{array}$ & $\begin{array}{l}9.5 \mathrm{E}-04 \\
1.3 \mathrm{E}-02 \\
8.5 \mathrm{E}-03 \\
4.8 \mathrm{E}-02\end{array}$ & $\begin{array}{l}9.5 \mathrm{E}-04 \\
1.3 \mathrm{E}-02 \\
8.5 \mathrm{E}-03 \\
4.8 \mathrm{E}-02\end{array}$ & $\begin{array}{l}1.8 \mathrm{E}-03 \\
1.9 \mathrm{E}-02 \\
1.1 \mathrm{E}-02 \\
5.7 \mathrm{E}-02\end{array}$ & $\begin{array}{l}4.5 \mathrm{E}-04 \\
5.0 \mathrm{E}-03 \\
3.6 \mathrm{E}-03 \\
2.2 \mathrm{E}-02\end{array}$ & $\begin{array}{l}9.6 \mathrm{E}-04 \\
1.3 \mathrm{E}-02 \\
8.5 \mathrm{E}-03 \\
4.8 \mathrm{E}-02\end{array}$ & $\begin{array}{l}9.7 \mathrm{E}-04 \\
1.3 \mathrm{E}-02 \\
8.8 \mathrm{E}-03 \\
4.9 \mathrm{E}-02\end{array}$ \\
\hline TOTAL & $7.1 \mathrm{E}-02$ & $7.1 \mathrm{E}-02$ & $8.8 \mathrm{E}-02$ & $3.1 \mathrm{E}-02$ & $7.1 \mathrm{E}-02$ & 7. $2 \mathrm{E}-02$ \\
\hline
\end{tabular}

Production/consumption factors:

Produce: <1 Milk: 7.5 Meat: 1.1

FRACTION OF POPULATION RECEIVING AN INOICATED AVERAGE

TOTAL-BODY DOSE COMMITMENT FROM AIRBORNE PATHWAYS

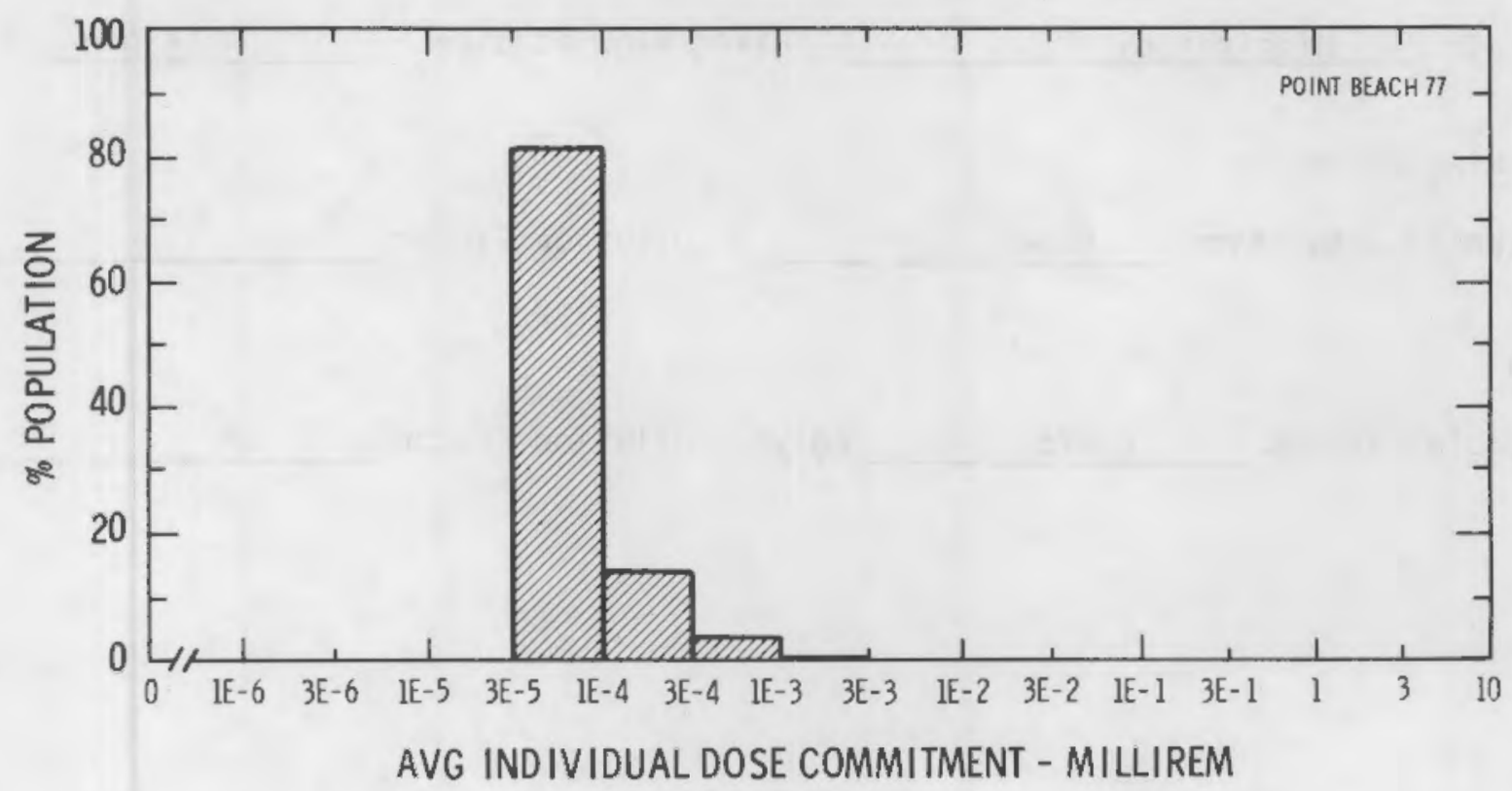


Site PRAIRIE ISLAND

Location $\mathrm{N}$ $44.6217^{\circ}$

Total Population Within 2-to-80-km Region
RED WING, MINNESOTA

$92.6330^{\circ}$

Major Metropolitan Centers Within Region

Center

Minneapolis-St. Paul SMSA

Rochester

Hastings
Population

2.0 6

60,000

13.000
Location

$50 \mathrm{~km}$

$64 \mathrm{~km}$

$19 \mathrm{~km}$

$\frac{60,000}{13,000}$

\begin{tabular}{rr}
$64 \mathrm{~km}$ & SSE \\
\hline $19 \mathrm{~km}$ & $\mathrm{NW}$ \\
\hline
\end{tabular}

Average Annual State Production of Crops and Animal Products in $80-\mathrm{km}$ Radius Circle Veg $1.2 \mathrm{E} 8$ $\mathrm{kg} \quad \mathrm{Milk} \quad 4.0 \mathrm{E} 8$ \& Meat $1.0 \mathrm{E} 8$ $\mathrm{kg}$

Regional Productivity Factor

Animal Grazing Factor 1

Location of Meteorological Station Site 0.5 Period of Record 22 MAR $74-21$ MAR 75

Average Mississippi River Flow at Site $15.000 \mathrm{ft}^{3} / \mathrm{s}$

Drinking Water Exposed Population None Dilution Factor Recovery $65 \%$

Fish

Edible Harvest $6.8 \mathrm{E} 5$ $\mathrm{kg} / \mathrm{yr}$ Dilution Factor 1 


Infant
Child
Teen
Adult
TOTAL

Inf ant Child Teen Adult TOTAL

Production/consumption factors:

Produce: <1 Milk: $1.4 \quad$ Meat: <1

Dose Commitments (person-rem) from Airborne Pathways

\begin{tabular}{|c|c|c|c|c|c|}
\hline Total Body & GI-LLI & Thyroid & Bone & Liver & Lung \\
\hline $\begin{array}{l}4.6 \mathrm{E}-03 \\
5.3 \mathrm{E}-02 \\
3.8 \mathrm{E}-02 \\
2.3 \mathrm{E}-01\end{array}$ & $\begin{array}{l}4.6 \mathrm{E}-03 \\
5.4 \mathrm{E}-02 \\
3.8 \mathrm{E}-02 \\
2.3 \mathrm{E}-01\end{array}$ & $\begin{array}{l}1.4 \mathrm{E}-02 \\
1.1 \mathrm{E}-01 \\
6.2 \mathrm{E}-02 \\
3.1 \mathrm{E}-01\end{array}$ & $\begin{array}{l}4.1 \mathrm{E}-03 \\
4.6 \mathrm{E}-02 \\
3.3 \mathrm{E}-02 \\
2.0 \mathrm{E}-01\end{array}$ & $\begin{array}{l}4.6 \mathrm{E}-03 \\
5.4 \mathrm{E}-02 \\
3.8 \mathrm{E}-02 \\
2.3 \mathrm{E}-01\end{array}$ & $\begin{array}{l}5.6 \mathrm{E}-03 \\
6.7 \mathrm{E}-02 \\
5.6 \mathrm{E}-02 \\
2.9 \mathrm{E}-01\end{array}$ \\
\hline $3.2 E-01$ & 3. $3 E-01$ & $5.0 E-01$ & $2.8 \mathrm{E}-01$ & 3. 3E-01 & 4.1E-01 \\
\hline
\end{tabular}

FRACTION OF POPULATION RECEIVING AN INOICATED AVERAGE

TOTAL-BODY DOSE COMMITMENT FROM AIRBORNE PATHWAYS

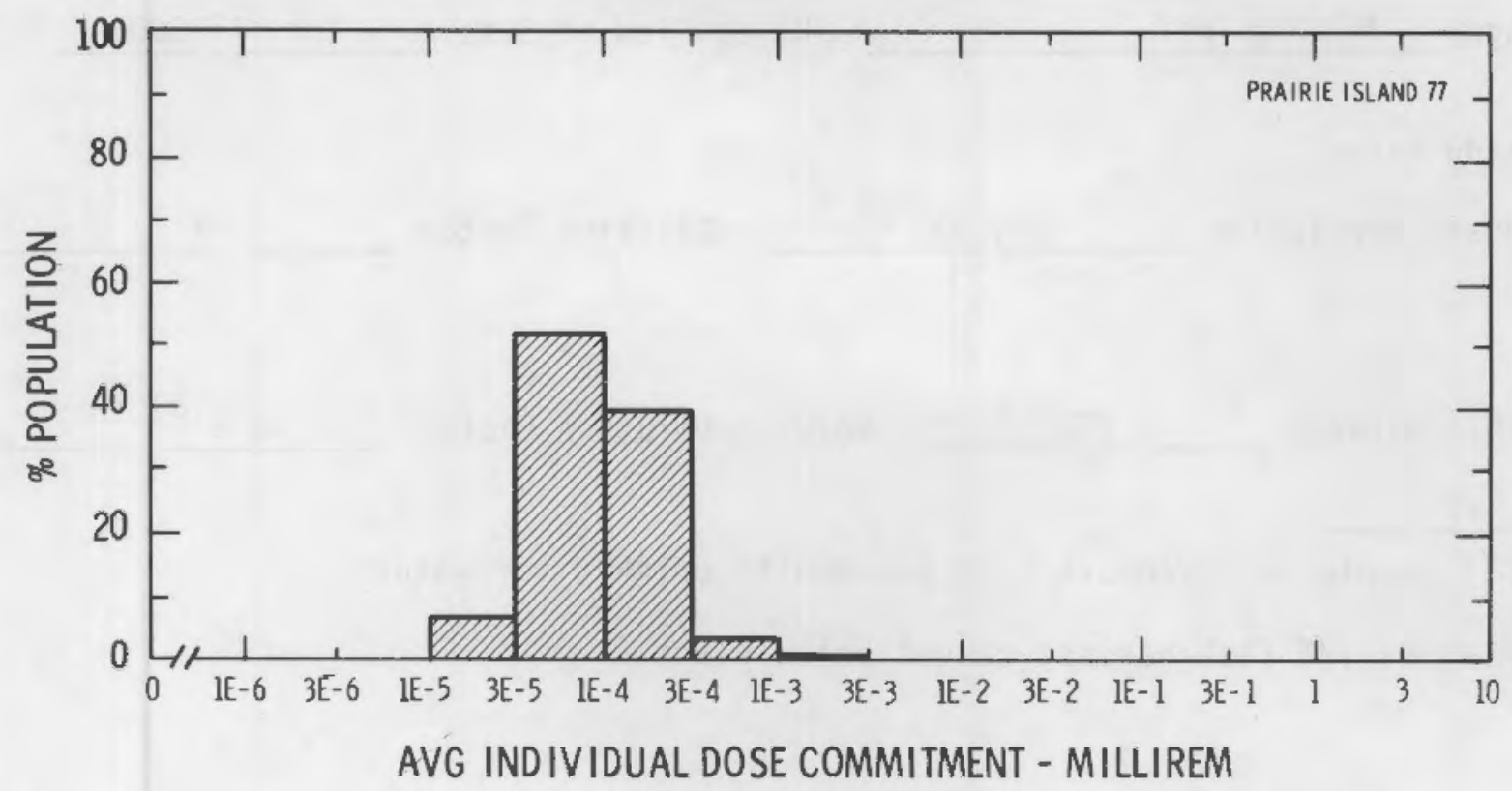


Site QUAD CITIES

Location $\mathrm{N}$

$41.7272^{\circ}$

Total Population Within 2-to-80-km Region
ROCK ISLAND, ILLINOIS

W $90.3417^{\circ}$

Major Metropolitan Centers Within Region

Center

Davenport SMSA
Population 360,000
Location

SW

Average Annual State Production of Crops and Animal Products in $80-\mathrm{km}$ Radius Circle Veg $1.1 \mathrm{E} 8{ }^{\mathrm{kg}}$ Milk $1.8 \mathrm{E} 8{ }^{\ell}$ Meat $1.9 \mathrm{E} 8 \mathrm{~kg}$

Regional Productivity Factor

Animal Grazing Factor

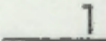

Location of Meteorological Station Site

0.5

Period of Record

1. JAN $74-31$ DEC 75

Average

Mississippi

River Flow at Site

47,000

$\mathrm{ft}^{3} / \mathrm{s}$

Drinking Water

Exposed Population

$370,000^{(a)}$

Dilution Factor

1

Fish

Edible Harvest

2.1E6 ${ }^{(b)}$ $\mathrm{kg} / \mathrm{yr}$

Dilution Factor

$0.5^{(b)}$

(a) All people in Davenport SMSA assumed to drink river water.

(b) Assumes $1 / 2$ fish harvest caught below plant. 


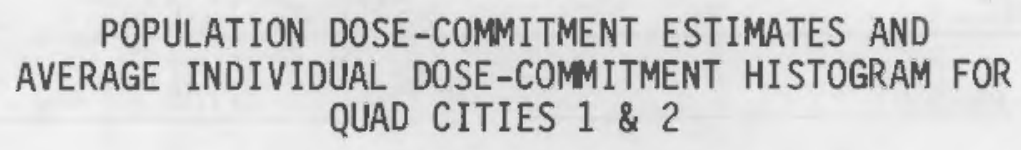

Dose Commitments (person-rem) from Liquid Pathways

\begin{tabular}{|c|c|c|c|c|c|}
\hline & Total Body & GI-LLI & Thyroid & Bone & Liver \\
\hline $\begin{array}{l}\text { Infant } \\
\text { Child } \\
\text { Teen } \\
\text { Adult }\end{array}$ & $\begin{array}{l}1.1 \mathrm{E}-03 \\
1.2 \mathrm{E}-01 \\
2.2 \mathrm{E}-01 \\
2.4 \mathrm{E}+00\end{array}$ & $\begin{array}{l}3.5 \mathrm{E}-04 \\
9.5 \mathrm{E}-03 \\
1.2 \mathrm{E}-02 \\
1.0 \mathrm{E}-01\end{array}$ & $\begin{array}{l}2.2 \mathrm{E}-02 \\
1.6 \mathrm{E}-01 \\
5.1 \mathrm{E}-02 \\
3.6 \mathrm{E}-01\end{array}$ & $\begin{array}{l}6.9 \mathrm{E}-03 \\
6.1 \mathrm{E}-01 \\
3.4 \mathrm{E}-01 \\
2.0 \mathrm{E}+00\end{array}$ & $\begin{array}{l}8.6 \mathrm{E}-03 \\
6.9 \mathrm{E}-01 \\
5.5 \mathrm{E}-01 \\
3.3 \mathrm{E}+00\end{array}$ \\
\hline TOTAL & 2. $7 \mathrm{E}+00$ & $1.2 \mathrm{E}-01$ & $5.9 \mathrm{E}-01$ & $3.0 \mathrm{E}+00$ & $4.5 E+00$ \\
\hline
\end{tabular}

Dose Commitments (person-rem) from Airborne Pathways

\begin{tabular}{|c|c|c|c|c|c|c|}
\hline & Total Body & GI-LLI & Thyroid & Bone & Liver & Lung \\
\hline $\begin{array}{l}\text { Inf ant } \\
\text { Child } \\
\text { Teen } \\
\text { Adult }\end{array}$ & $\begin{array}{l}1.8 \mathrm{E}-02 \\
2.1 \mathrm{E}-01 \\
1.5 \mathrm{E}-01 \\
9.1 \mathrm{E}-01\end{array}$ & $\begin{array}{l}1.8 \mathrm{E}-02 \\
2.0 \mathrm{E}-01 \\
1.5 \mathrm{E}-01 \\
9.1 \mathrm{E}-01\end{array}$ & $\begin{array}{l}2.9 E-01 \\
2.8 E+00 \\
1.2 E+00 \\
4.7 E+00\end{array}$ & $\begin{array}{l}2.0 \mathrm{E}-02 \\
2.8 \mathrm{E}-01 \\
1.7 \mathrm{E}-01 \\
9.7 \mathrm{E}-01\end{array}$ & $\begin{array}{l}2.0 \mathrm{E}-02 \\
2.2 \mathrm{E}-01 \\
1.5 \mathrm{E}-01 \\
9.1 \mathrm{E}-01\end{array}$ & $\begin{array}{l}1.9 \mathrm{E}-02 \\
2.1 \mathrm{E}-01 \\
1.6 \mathrm{E}-01 \\
9.4 \mathrm{E}-01\end{array}$ \\
\hline TOTAL & 1. $3 \mathrm{E}+00$ & $1.3 \mathrm{E}+00$ & $9.1 \mathrm{E}+00$ & $1.4 E+00$ & $1.3 E+00$ & 1. $3 E+00$ \\
\hline
\end{tabular}

Production/consumption factors:
Produce: $<1$
Milk: 2.0
Meat: 3.5

FRACTION OF POPULATION RECEIVING AN INDICATED AVERAGE TOTAL-BODY DOSE COMMITMENT FROM AIRBORNE PATHWAYS

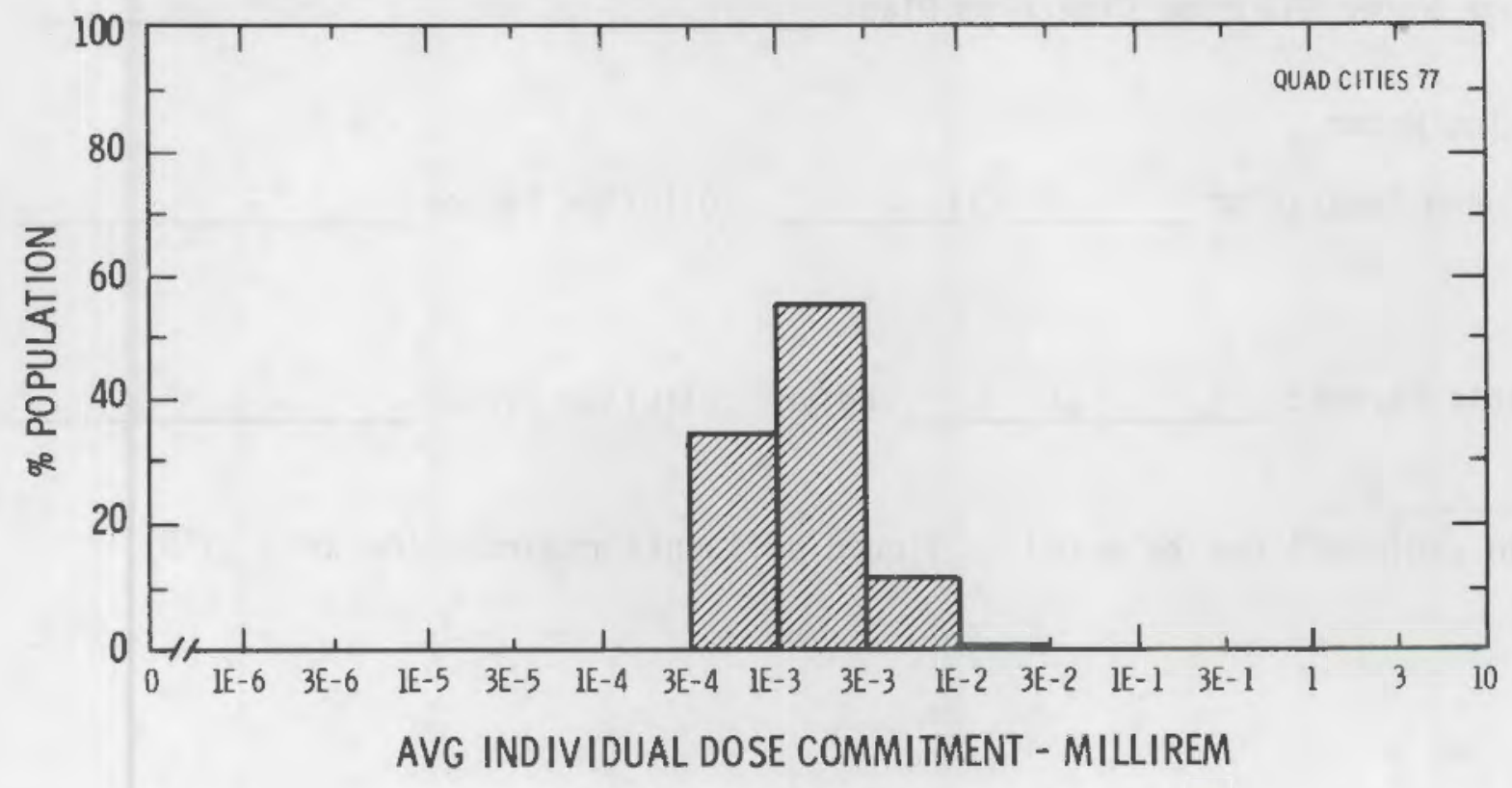


Site RANCHO SECO

Location N $38.3461^{\circ}$

Total Population Within 2-to-80-km Region
SACRAMENTO COUNTY, CALIFORNIA $W$ $121.1186^{\circ}$

Major Metropolitan Centers Within Region

Center

Sacramento

Stockton
Population

$\begin{array}{r}870,000 \\ \hline 120,000 \\ \hline\end{array}$

Location

\begin{tabular}{ll}
$35 \mathrm{~km}$ & $\mathrm{NNW}$ \\
\hline $45 \mathrm{~km}$ & $\mathrm{SW}$ \\
\hline
\end{tabular}

Average Annual State Production of Crops and Animal Products in $80-\mathrm{km}$ Radius Circle

Veg $\frac{4.8 \mathrm{E} 7}{\mathrm{~kg} M i \mathrm{MK} \_2.3 \mathrm{E} 8{ }^{\ell} \text { Meat } 5.0 \mathrm{E} 7} \mathrm{~kg}$

Regional Productivity Factor

Animal Grazing Factor 1

Location of Meteorological Station Site 0.9

Period of Record 1 FEB $75-31$ JAN 76

Average Water Dilution Flow from Plant (a) $\mathrm{ft}^{3} / \mathrm{s}$

Orinking Water Exposed Population

(a) Dilution Factor Recovery $\quad 98 \%$

Fish

Edible Harvest (a) $\mathrm{kg} / \mathrm{yr}$ Dilution Factor $-$

(a) No radionuclides released in liquid effluents reported (Decker, 1979). 


\section{POPULATION DOSE-COMMITMENT ESTIMATES AND AVERAGE INDIVIDUAL. DOSE-COMMITMENT HISTOGRAM FOR RANCHO SECO}

\section{TOTAL}

Inf ant
Child
Teen
Adult
TOTAL

Dose Commitments (person-rem) from Liquid Pathways

$\frac{\text { Total Body }}{0} \quad \frac{\text { GI-LLI }}{0} \quad \frac{\text { Thyroid }}{0} \quad \frac{\text { Bone }}{0} \quad \frac{\text { Liver }}{0}$

Dose Commitments (person-rem) from Airborne Pathways

\begin{tabular}{|c|c|c|c|c|c|}
\hline Total Body & GI-LLI & Thyroid & Bone & Liver & Lung \\
\hline $\begin{array}{l}9.3 \mathrm{E}-04 \\
1.1 \mathrm{E}-02 \\
7.6 \mathrm{E}-03 \\
4.5 \mathrm{E}-02\end{array}$ & $\begin{array}{l}9.2 \mathrm{E}-04 \\
1.1 \mathrm{E}-02 \\
7.5 \mathrm{E}-03 \\
4.5 \mathrm{E}-02\end{array}$ & $\begin{array}{l}3.2 \mathrm{E}-03 \\
2.3 \mathrm{E}-02 \\
1.2 \mathrm{E}-02 \\
6.0 \mathrm{E}-02\end{array}$ & $\begin{array}{l}8.2 \mathrm{E}-04 \\
9.2 \mathrm{E}-03 \\
6.6 \mathrm{E}-03 \\
4.0 \mathrm{E}-02\end{array}$ & $\begin{array}{l}9.3 \mathrm{E}-04 \\
1.1 \mathrm{E}-02 \\
7.6 \mathrm{E}-03 \\
4.5 \mathrm{E}-02\end{array}$ & $\begin{array}{l}9.8 \mathrm{E}-04 \\
1.1 \mathrm{E}-02 \\
8.7 \mathrm{E}-03 \\
4.8 \mathrm{E}-02\end{array}$ \\
\hline $6.4 E-02$ & $6.4 \mathrm{E}-02$ & $9.8 \mathrm{E}-02$ & $5.7 E-02$ & $6.4 E-02$ & 7.0E-02 \\
\hline
\end{tabular}

Production/consumption factors:

Produce: <1 Milk: 1.2 Meat: <1

FRACTION OF POPULATION RECEIVING AN INDICATED AVERAGE TOTAL-BODY DOSE COMMITMENT FROM AIRBORNE PATHWAYS

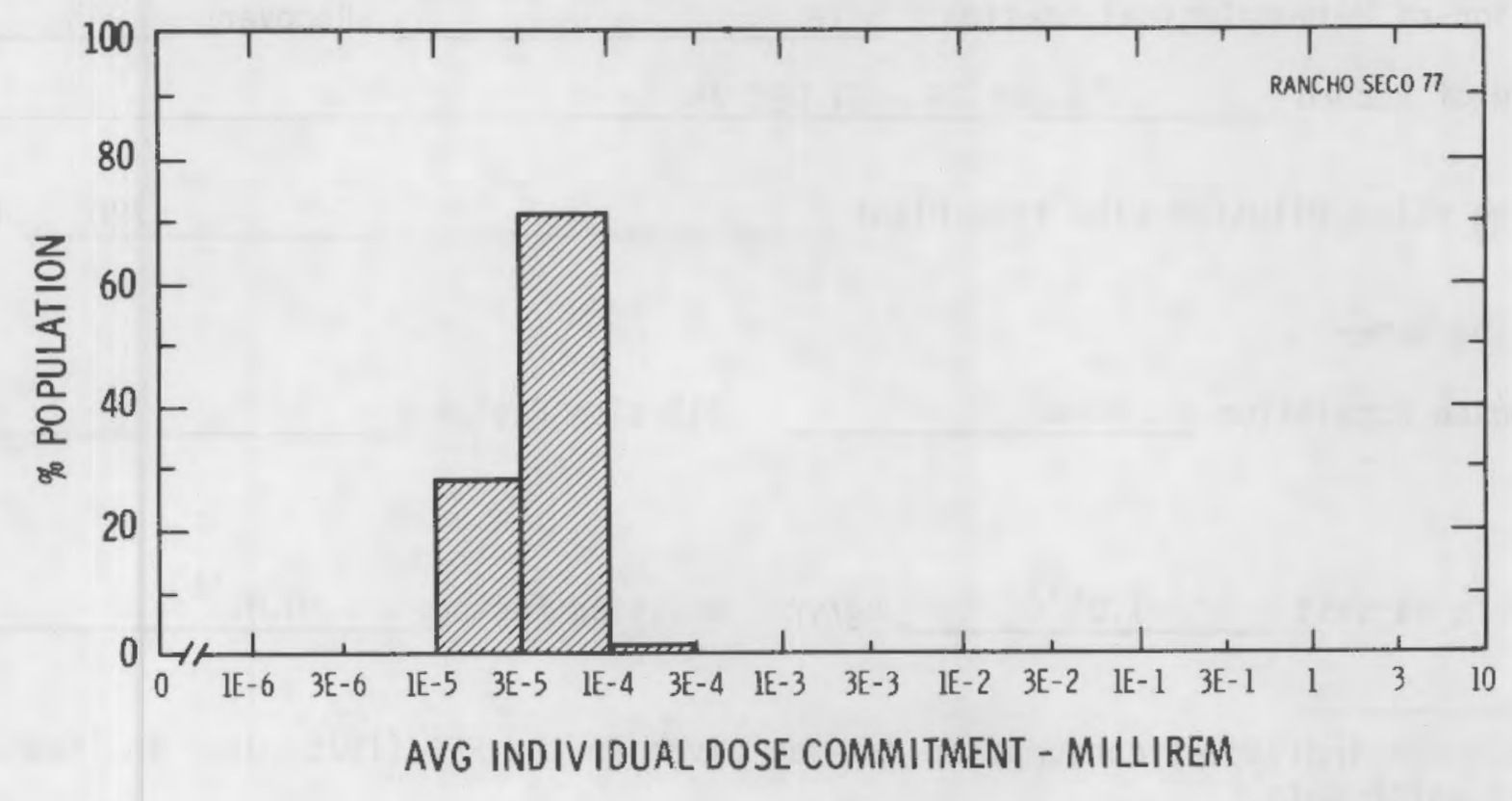


Site H. B. ROBINSON Location $\mathrm{N}$ $34.4014^{\circ}$

Total Population Within 2-to-80-km Region
HARTSVILLE, SOUTH CAROLINA

W $\mathrm{B} 0.1567^{\circ}$

\section{$6.4 \mathrm{E} 5$}

Major Metropolitan Centers Within Region

\begin{tabular}{c}
$\frac{\begin{array}{c}\text { Center } \\
\text { Florence }\end{array}}{\text { Sumter }}-\frac{\frac{\text { Population }}{29,000}}{27,000}$ \\
\hline
\end{tabular}

\begin{tabular}{rr}
\multicolumn{2}{c}{ Location } \\
$40 \mathrm{~km}$ & SE \\
\hline $53 \mathrm{~km}$ & SSW \\
\hline
\end{tabular}

Average Annual State Production of Crops and Animal Products in $80-\mathrm{km}$ Radius Circle Veg $7.4 \mathrm{E} 6 \quad \mathrm{~kg} \mathrm{Milk} 5.7 \mathrm{E} 7 \_$Meat $5.0 \mathrm{E} 7$

Regional Productivity Factor

Animal Grazing Factor

Site _. Recovery $\frac{1}{\frac{0.8}{94 \%}}$

Location of Meteorological Station Site.. Period of Record 1 JAN $75-31$ DEC 75

Average Water Dilution Flow from Plant $720 \quad \mathrm{ft}^{3} / \mathrm{s}$

Drinking Water

Exposed Fopulation None Dilution Factor

Fish
Edible Harvest
$1.8^{(a)}$ $\mathrm{kg} / \mathrm{yr}$ Dilution Factor $0.02^{(b)}$

(a) Average individual consumption rate as given in the FES (1975) used in lieu of catch data.

(b) Ten percent of population consumes fish taken from water diluted by a factor of 0.2 (FES, 1975). 
POPULATION DOSE-COMMITMENT ESTIMATES AND

AVERAGE INDIVIDUAL DOSE-COMMITMENT HISTOGRAM FOR

H. B. ROBINSON

Dose Commitments (person-rem) from Liquid Pathways

\begin{tabular}{|c|c|c|c|c|c|}
\hline & Total Body & GI-LLI & Thyroid & Bone & Liver \\
\hline $\begin{array}{l}\text { Infant } \\
\text { Child } \\
\text { Teen } \\
\text { Adult }\end{array}$ & $\begin{array}{l}0.0 \mathrm{E}+00 \\
1.9 \mathrm{E}-02 \\
3.7 \mathrm{E}-02 \\
3.9 \mathrm{E}-01\end{array}$ & $\begin{array}{l}0.0 \mathrm{E}+00 \\
2.0 \mathrm{E}-03 \\
4.0 \mathrm{E}-03 \\
3.4 \mathrm{E}-02\end{array}$ & $\begin{array}{l}0.0 E+00 \\
3.4 E-04 \\
2.9 E-04 \\
2.2 E-03\end{array}$ & $\begin{array}{l}0.0 \mathrm{E}+00 \\
8.7 \mathrm{E}-02 \\
5.3 \mathrm{E}-02 \\
3.1 \mathrm{E}-01\end{array}$ & $\begin{array}{l}0.0 \mathrm{E}+00 \\
1.0 \mathrm{E}-01 \\
9.0 \mathrm{E}-02 \\
5.3 \mathrm{E}-01\end{array}$ \\
\hline TOTAL & 4. $5 E-01$ & $4.0 \mathrm{E}-02$ & $2.8 \mathrm{E}-03$ & $4.5 \mathrm{E}-01$ & 7. $3 \mathrm{E}-01$ \\
\hline
\end{tabular}

Dose Commitments (person-rem) from Airborne Pathways

\begin{tabular}{|c|c|c|c|c|c|c|}
\hline & Total Body & GI-LLI & Thyroid & Bone & Liver & Lung \\
\hline $\begin{array}{l}\text { Infant } \\
\text { Child } \\
\text { Teen } \\
\text { Adult }\end{array}$ & $\begin{array}{l}3.1 \mathrm{E}-04 \\
4.1 \mathrm{E}-03 \\
2.8 \mathrm{E}-03 \\
1.7 \mathrm{E}-02\end{array}$ & $\begin{array}{l}3.1 \mathrm{E}-04 \\
4.1 \mathrm{E}-03 \\
2.8 \mathrm{E}-03 \\
1.7 \mathrm{E}-02\end{array}$ & $\begin{array}{l}1.7 \mathrm{E}-03 \\
1.2 \mathrm{E}-02 \\
6.1 \mathrm{E}-03 \\
2.8 \mathrm{E}-02\end{array}$ & $\begin{array}{l}1.5 \mathrm{E}-04 \\
1.6 \mathrm{E}-03 \\
1.2 \mathrm{E}-03 \\
7.1 \mathrm{E}-03\end{array}$ & $\begin{array}{l}3.1 E-04 \\
4.1 E-03 \\
2.8 E-03 \\
1.7 E-02\end{array}$ & $\begin{array}{l}3.2 \mathrm{E}-04 \\
4.2 \mathrm{E}-03 \\
3.0 \mathrm{E}-03 \\
1.7 \mathrm{E}-02\end{array}$ \\
\hline JTAL & $2.4 E-02$ & $2.4 \mathrm{E}-02$ & $4.8 \mathrm{E}-02$ & $1.0 E-02$ & $2.4 E-02$ & $2.5 \mathrm{E}-02$ \\
\hline
\end{tabular}

Production/consumption factors:

Produce: <1 Milk: <1 Meat: <1

FRACTION OF POPULATION RECEIVING AN INDICATED AVERAGE

TOTAL-BODY DOSE COMMITMENT FROM AIRBORNE PATHWAYS

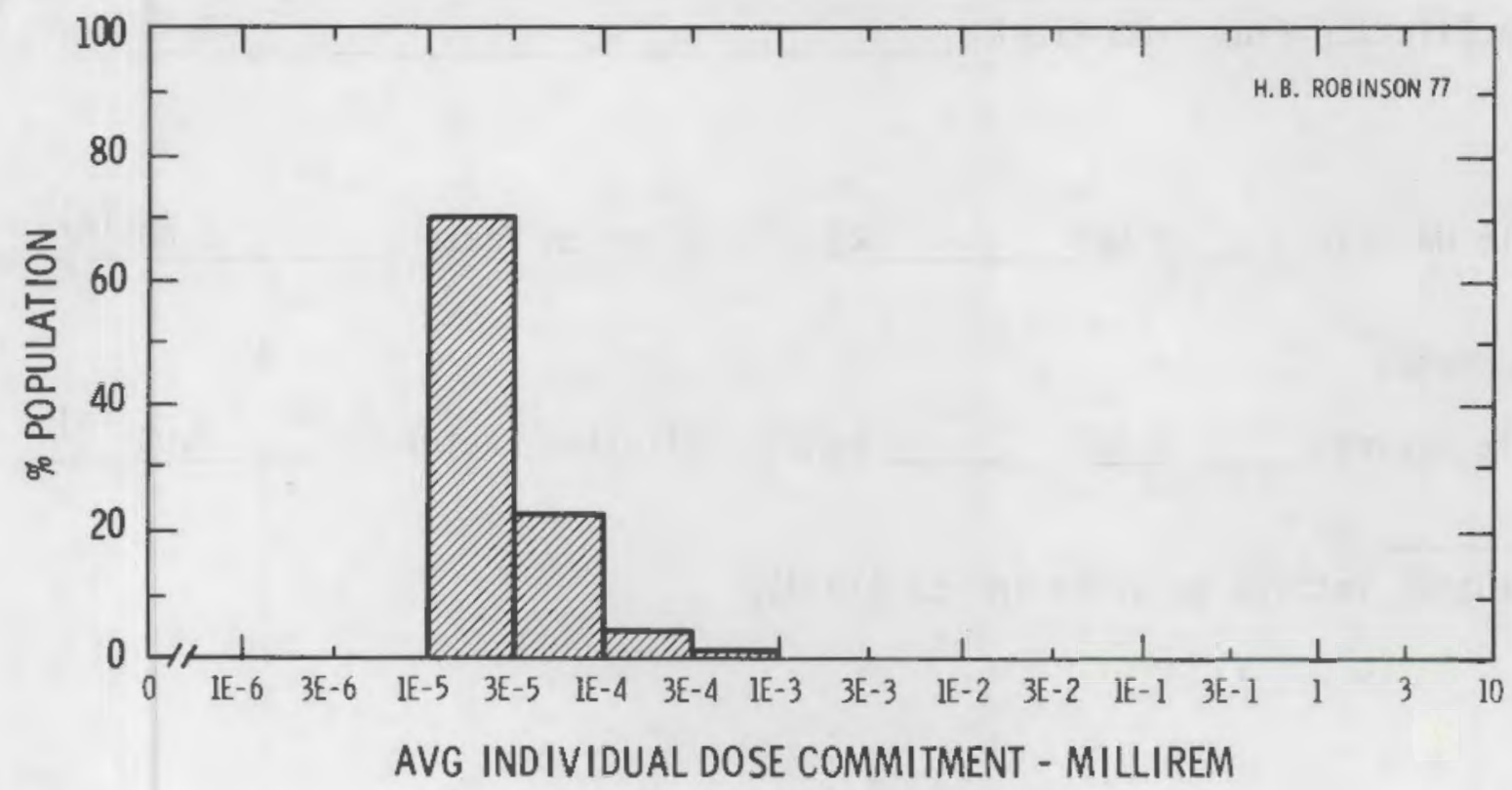


Site

ST. LUCIE

Location $\mathrm{N}$

$27.4466^{\circ}$

Total Population Within 2-to-80-km Region
FORT PIERCE, FLORIDA

W $80.3266^{\circ}$

Major Metropolitan Centers Within Region

\begin{tabular}{l}
$\frac{\text { Center }}{\text { West Palm Beach }}$ \\
\hline Ft. Pierce \\
\hline Vero Beach \\
\hline
\end{tabular}

\begin{tabular}{c}
$\frac{\text { Population }}{71,000}$ \\
\hline 37,000 \\
\hline 15,000 \\
\hline
\end{tabular}

\begin{tabular}{ll}
\multicolumn{1}{l}{$\frac{\text { Location }}{80 \mathrm{~km}}$} & SSE \\
\hline $13 \mathrm{~km}$ & $\mathrm{NW}$ \\
\hline $40 \mathrm{~km}$ & $\mathrm{NNW}$ \\
\hline
\end{tabular}

Average Annual State Production of Crops and Animal Products in $80-\mathrm{km}$ Radius Circle Veg $\mathrm{kg}$ Milk $1.1 E 8$ \& Meat $\mathrm{kg}$

Regional Productivity Factor 0.5 Animal Grazing Factor Location of Meteorological Station Site Recovery $92 \%$ Period of Record 1 JAN 76 - 31 DEC 76

Average Dilution Flow from Plant $41 \quad \mathrm{ft}^{3} / \mathrm{s}$

Fish

Edible Harvest 2.6E5 $\mathrm{kg} / \mathrm{yr}$ Dilution Factor $0.005^{(a)}$

Invertebrates

Edible Harvest 2.7E4 $\mathrm{kg} / \mathrm{yr}$ Dilution Factor $0.005^{(a)}$

(a) Dilution factors as given in FES (1973). 
POPULATION OOSE-COMMITMENT ESTIMATES ANO

AVERAGE INOIVIDUAL OOSE-COMMITMENT HISTOGRAM FOR

ST. LUCIE

Oose Commitments (person-rem) from Liquid Pathways

Inf ant

$\frac{\text { Total Body }}{0.0 \mathrm{E}+00} \quad \frac{\text { GI-LLI }}{0.0 \mathrm{E}+00}$

Thyroid

Bone

$\frac{\text { Liver }}{0.0 \mathrm{E}+00}$

Child

Teen

Adult

$7.0 \mathrm{E}-03$

2.6E-02

$5.8 \mathrm{E}-02$

$1.3 \mathrm{E}-02$

4.1E-02

9.3E-03

2.7E-01

$6.0 \mathrm{E}-02$

$1.1 \mathrm{E}-02$

$5.3 \mathrm{E}-02$

$4.8 \mathrm{E}-01$

3.7E-01

8.3E-02

$9.4 \mathrm{E}-03$

$6.7 \mathrm{E}-02$

$5.6 \mathrm{E}-01$

\begin{abstract}
8.3E-02
\end{abstract}
$5.6 \mathrm{E}-02$

Dose Cormitments (person-rem) from Airborne Pathways

\begin{tabular}{|c|c|c|c|c|c|c|}
\hline & Total Body & GI-LLI & Thyroid & Bone & Liver & Lung \\
\hline $\begin{array}{l}\text { Infant } \\
\text { Child } \\
\text { Teen } \\
\text { Adult }\end{array}$ & $\begin{array}{l}8.1 \mathrm{E}-03 \\
9.4 \mathrm{E}-02 \\
6.9 \mathrm{E}-02 \\
4.1 \mathrm{E}-01\end{array}$ & $\begin{array}{l}8.1 \mathrm{E}-03 \\
9.4 \mathrm{E}-02 \\
6.9 \mathrm{E}-02 \\
4.1 \mathrm{E}-01\end{array}$ & $\begin{array}{l}2.8 \mathrm{E}-02 \\
2.1 \mathrm{E}-01 \\
1.2 \mathrm{E}-01 \\
6.0 \mathrm{E}-01\end{array}$ & $\begin{array}{l}7.5 \mathrm{E}-03 \\
8.4 \mathrm{E}-02 \\
6.1 \mathrm{E}-02 \\
3.7 \mathrm{E}-01\end{array}$ & $\begin{array}{l}8.2 E-03 \\
9.4 E-02 \\
6.9 E-02 \\
4.1 E-01\end{array}$ & $\begin{array}{l}8.7 E-03 \\
1.0 E-01 \\
8.0 E-02 \\
4.5 E-01\end{array}$ \\
\hline TOTAL & $5.8 \mathrm{E}-01$ & $5.8 \mathrm{E}-01$ & $9.6 \mathrm{E}-01$ & $5.2 E-01$ & $5.9 \mathrm{E}-01$ & $6.4 \mathrm{E}-01$ \\
\hline
\end{tabular}

Production/consumption factors:

Produce: $<1$

Milk: 1.5

Meat: 1.6

FRACTION OF POPULATION RECEIVING AN INDICATED AVERAGE TOTAL-BODY DOSE COMMITMENT FROM AIRBORNE PATHWAYS

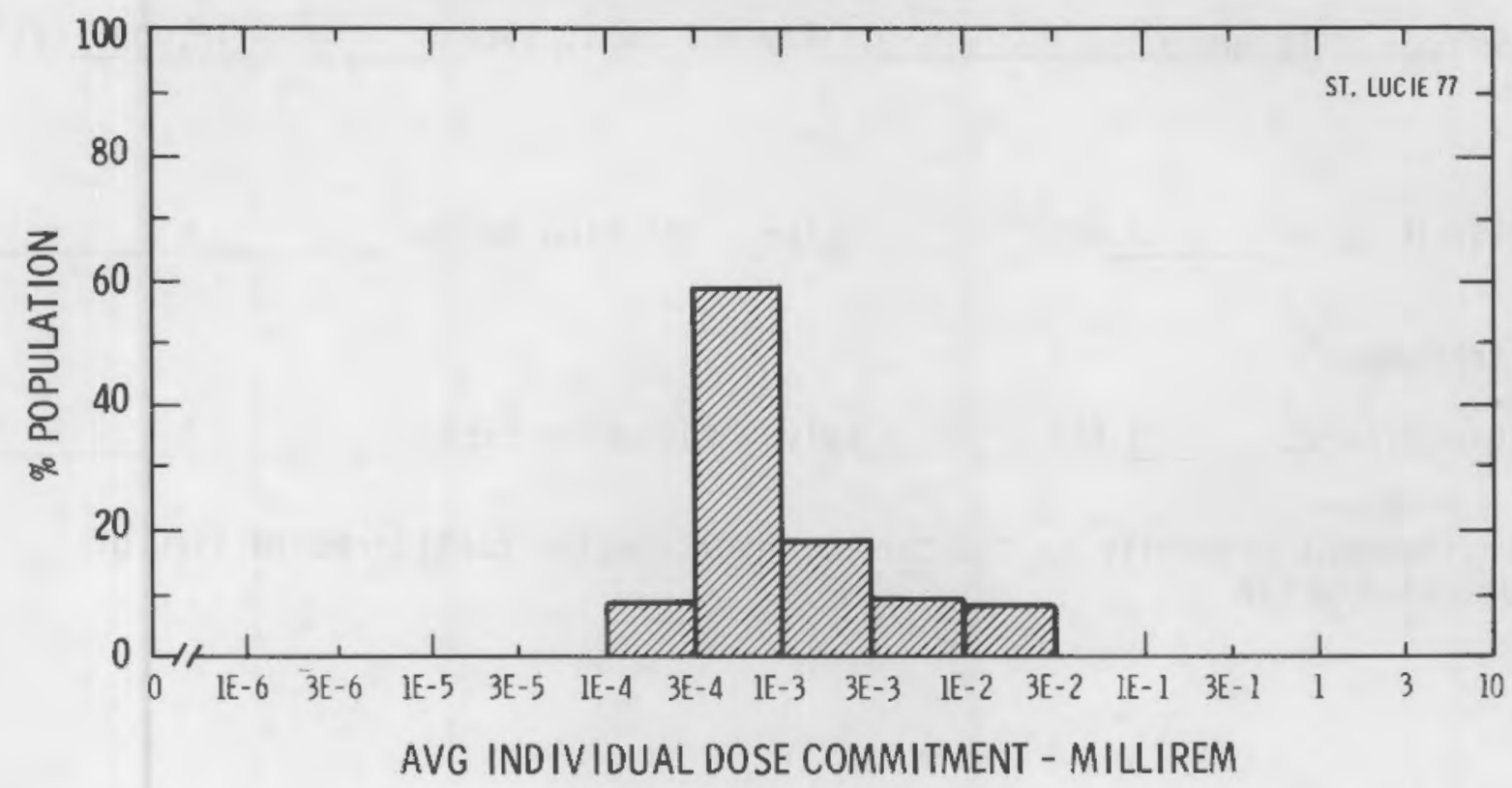


Site

SALEM

Location $\mathrm{N}$ $39.4628^{\circ}$

Total Population Within 2-to-80-km Region

Major Metropolitan Centers Within Region

\begin{tabular}{l}
\multicolumn{1}{c}{ Center } \\
Philadelphia SMSA \\
\hline Wilmington \\
\hline Chester \\
\hline
\end{tabular}

$\begin{array}{r}\frac{\text { Population }}{4,850,000} \\ \hline 501,000 \\ \hline 56,600 \\ \hline\end{array}$

Population

Location

Average Annual State Production of Crops and Animal Products in $80-\mathrm{km}$ Radius Circle Veg $\quad 7.4 \mathrm{E} 7 \mathrm{~kg}$ Milk 2.7E8 \& Meat 2.4E7 $\mathrm{kg}$

Regiona] Productivity Factor

\begin{tabular}{cc}
$64 \mathrm{~km}$ & $\mathrm{NE}$ \\
\hline $29 \mathrm{~km}$ & $\mathrm{~N}$ \\
\hline $45 \mathrm{~km}$ & $\mathrm{NNE}$ \\
\hline
\end{tabular}

Animal Grazing Factor 0.9

Location of Meteorological Station Site 0.6

Period of Record 1 JUN 70 - 31 MAY 71

Average Delaware

River Flow at Site $16,000 \mathrm{ft}^{3} / \mathrm{s}$

Fish

Edible Harvest 3.6E5 $\mathrm{kg} / \mathrm{yr}$ Dilution Factor 1 Invertebrates ${ }^{(a)}$ Edible Harvest $1.6 \mathrm{E} 5$ $\mathrm{kg} / \mathrm{yr}$ Dilution Factor 1

(a) Environment primarily salt water so invertebrates considered in lieu of drinking water. 


\begin{abstract}
POPULATION DOSE-COMMITMENT ESTIMATES AND AVERAGE INDIVIDUAL DOSE-COMMITMENT HISTOGRAM FOR SALEM
\end{abstract}

Infant
Child
Teen
Adult
TOTAL

Dose Commitments (person-rem) from Liquid Pathways

\begin{tabular}{|c|c|c|c|c|}
\hline Total Body & GI-LLI & Thyroid & Bone & Liver \\
\hline $\begin{array}{l}0.0 \mathrm{E}+00 \\
1.5 \mathrm{E}-02 \\
1.0 \mathrm{E}-02 \\
6.2 \mathrm{E}-02\end{array}$ & $\begin{array}{l}0.0 \mathrm{E}+00 \\
3.0 \mathrm{E}-02 \\
6.3 \mathrm{E}-02 \\
5.6 \mathrm{E}-01\end{array}$ & $\begin{array}{l}0.0 \mathrm{E}+00 \\
5.6 \mathrm{E}-04 \\
4.1 \mathrm{E}-04 \\
2.9 \mathrm{E}-03\end{array}$ & $\begin{array}{l}0.0 E+00 \\
4.3 E-03 \\
2.6 E-03 \\
1.6 E-02\end{array}$ & $\begin{array}{l}0.0 \mathrm{E}+00 \\
1.3 \mathrm{E}-02 \\
1.1 \mathrm{E}-02 \\
7.0 \mathrm{E}-02\end{array}$ \\
\hline $8.7 E-02$ & $6.5 \mathrm{E}-01$ & $3.8 \mathrm{E}-03$ & 2. $3 E-02$ & $9.4 \mathrm{E}-02$ \\
\hline
\end{tabular}

Dose Commitments (person-rem) from Airborne Pathways

\begin{tabular}{|c|c|c|c|c|c|c|}
\hline & Total Body & GI-LLI & Thyroid & Bone & Liver & Lung \\
\hline $\begin{array}{l}\text { Infant } \\
\text { Child } \\
\text { Teen } \\
\text { Adult }\end{array}$ & $\begin{array}{l}4.6 E-04 \\
6.8 E-03 \\
4.7 E-03 \\
2.6 E-02\end{array}$ & $\begin{array}{l}4.6 \mathrm{E}-04 \\
6.8 \mathrm{E}-03 \\
4.6 \mathrm{E}-03 \\
2.6 \mathrm{E}-02\end{array}$ & $\begin{array}{l}4.6 \mathrm{E}-04 \\
6.8 \mathrm{E}-03 \\
4.6 \mathrm{E}-03 \\
2.6 \mathrm{E}-02\end{array}$ & $\begin{array}{l}2.9 \mathrm{E}-05 \\
3.6 \mathrm{E}-04 \\
2.3 \mathrm{E}-04 \\
1.2 \mathrm{E}-03\end{array}$ & $\begin{array}{l}4.6 \mathrm{E}-04 \\
6.8 \mathrm{E}-03 \\
4.6 \mathrm{E}-03 \\
2.6 \mathrm{E}-02\end{array}$ & $\begin{array}{l}4.6 \mathrm{E}-04 \\
6.8 \mathrm{E}-03 \\
4.7 \mathrm{E}-03 \\
2.6 \mathrm{E}-02\end{array}$ \\
\hline TOTAL & $3.8 \mathrm{E}-02$ & 3. $7 \mathrm{E}-02$ & 3. $7 \mathrm{E}-02$ & $1.8 \mathrm{E}-03$ & $3.7 E-02$ & $3.8 E-02$ \\
\hline
\end{tabular}

Production/consumption factors:

Produce: $<1$

Milk: $<1$

Meat: $<1$

FRACTION OF POPULATIDN RECEIVING AN INDICATED AVERAGE

TOTAL-BODY DOSE COMMITMENT FROM AIRBORNE PATHWAYS

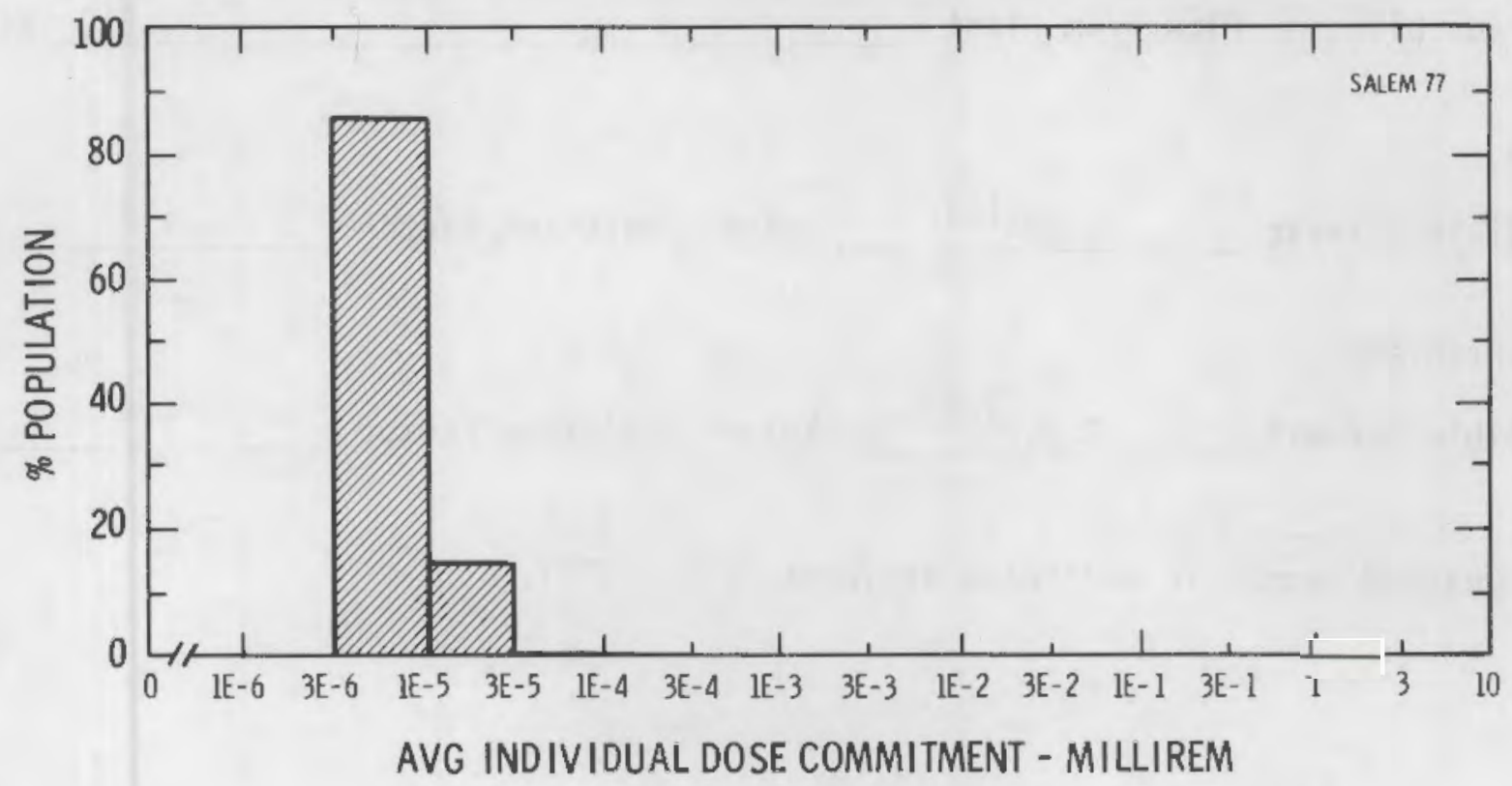


Site SAN ONOFRE

Location

N

$33.3686^{\circ}$

Total Population Within 2-to-80-km Region
CAMP PENDLETON, CALIFORNIA

W $117.5544^{\circ}$

3.8E6

Major Metropolitan Centers Within Region

\begin{tabular}{l}
$\frac{\text { Center }}{\text { San Diego SMSA }}$ \\
\hline Riverside \\
\hline Anaheim \\
\hline Santa Ana \\
\hline
\end{tabular}

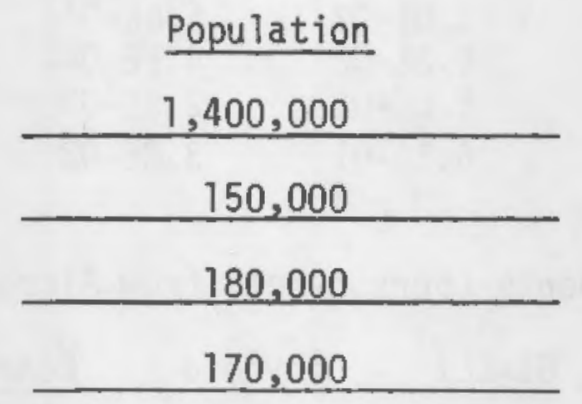

\begin{tabular}{cc}
\multicolumn{2}{c}{ Location } \\
$68 \mathrm{~km}$ & SSE \\
\hline $69 \mathrm{~km}$ & $\mathrm{NE}$ \\
\hline $69 \mathrm{~km}$ & $\mathrm{NNW}$ \\
\hline $55 \mathrm{~km}$ & $\mathrm{NNW}$ \\
\hline
\end{tabular}

Average Annual State Production of Crops and Animal Products in $80-\mathrm{km}$ Radius Circle

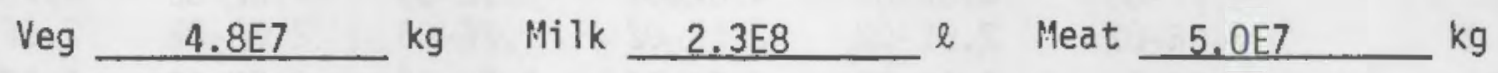

Regional Productivity Factor

Animal Grazing Factor

Location of Meteorological Station Site

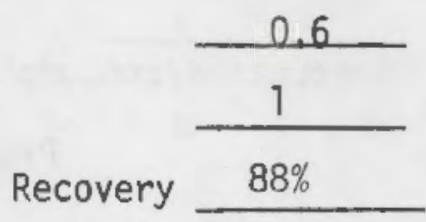

Period of Record 25 JAN $73-24$ JAN 76

Average Dilution Flow from Plant $510 \mathrm{ft}^{3} / \mathrm{s}$

Fish

Edible Harvest $2.9 \mathrm{E} 4^{(\mathrm{a})}$ $\mathrm{kg} / \mathrm{yr}$ Dilution Factor I(a)

Invertebrates

Edible Harvest 2. $9 \mathrm{E} 3^{(\mathrm{a})}$ $\mathrm{kg} / \mathrm{yr}$ Dilution Factor ${ }_{1}^{(a)}$

(a) Seafood caught in undiluted effluent (FES, 1973). 


\section{POPULATION OOSE-COMMITMENT ESTIMATES AND AVERAGE INDIVIOUAL DOSE-COMMITMENT HISTOGRAM FOR SAN ONOFRE}

Dose Commitments (person-rem) from Liquid Pathways

Inf ant

Child

Teen

Adult

TOTAL
Infant

Child

Teen

Adult

TOTAL

\begin{tabular}{l} 
Total Body \\
\hline $0.0 \mathrm{E}+00$ \\
$4.6 \mathrm{E}-02$ \\
$6.4 \mathrm{E}-02$ \\
$6.3 \mathrm{E}-01$ \\
$7.4 \mathrm{E}-01$
\end{tabular}

$\frac{\text { Thyroid }}{0.0 \mathrm{E}+00}$

3. $9 \mathrm{E}-03$

3. $0 \mathrm{E}-03$

2.1E-02

2.8E-02 $\frac{\text { Bone }}{0.0 E+00}$

$1.5 \mathrm{E}-01$

$8.8 \mathrm{E}-02$

$5.1 E-01$

7.5E-01 $\frac{\text { Liver }}{0.0 \mathrm{E}+00}$

1.7E-01

1.5E-01

8.7E-01

$1.2 \mathrm{E}+00$

Oose Commitments (person-rem) from Airborne Pathways

\begin{tabular}{|c|c|c|c|c|c|}
\hline Total Body & GI-LLI & Thyroid & Bone & Liver & Lung \\
\hline $\begin{array}{l}4.3 \mathrm{E}-04 \\
6.1 \mathrm{E}-03 \\
4.3 \mathrm{E}-03 \\
2.5 \mathrm{E}-02\end{array}$ & $\begin{array}{l}4.3 E-04 \\
6.1 E-03 \\
4.3 E-03 \\
2.5 E-02\end{array}$ & $\begin{array}{l}6.4 \mathrm{E}-04 \\
7.3 \mathrm{E}-03 \\
4.8 \mathrm{E}-03 \\
2.6 \mathrm{E}-02\end{array}$ & $\begin{array}{l}8.1 \mathrm{E}-05 \\
9.0 \mathrm{E}-04 \\
6.6 \mathrm{E}-04 \\
4.0 \mathrm{E}-03\end{array}$ & $\begin{array}{l}4.3 \mathrm{E}-04 \\
6.1 \mathrm{E}-03 \\
4.3 \mathrm{E}-03 \\
2.5 \mathrm{E}-02\end{array}$ & $\begin{array}{l}4.3 \mathrm{E}-04 \\
6.2 \mathrm{E}-03 \\
4.5 \mathrm{E}-03 \\
2.5 \mathrm{E}-02\end{array}$ \\
\hline $3.6 \mathrm{E}-02$ & $3.6 E-02$ & $3.9 \mathrm{E}-02$ & $5.6 \mathrm{E}-03$ & $3.6 \mathrm{E}-02$ & 3. $6 \mathrm{E}-02$ \\
\hline
\end{tabular}

Production/consumption factors:

Produce: $<1$

Milk: $<1$

Meat: $<1$

FRACTION OF POPULATION RECEIVING AN INDICATED AVERAGE

TOTAL-BODY DOSE COMMITMENT FROM AIRBORNE PATHWAYS

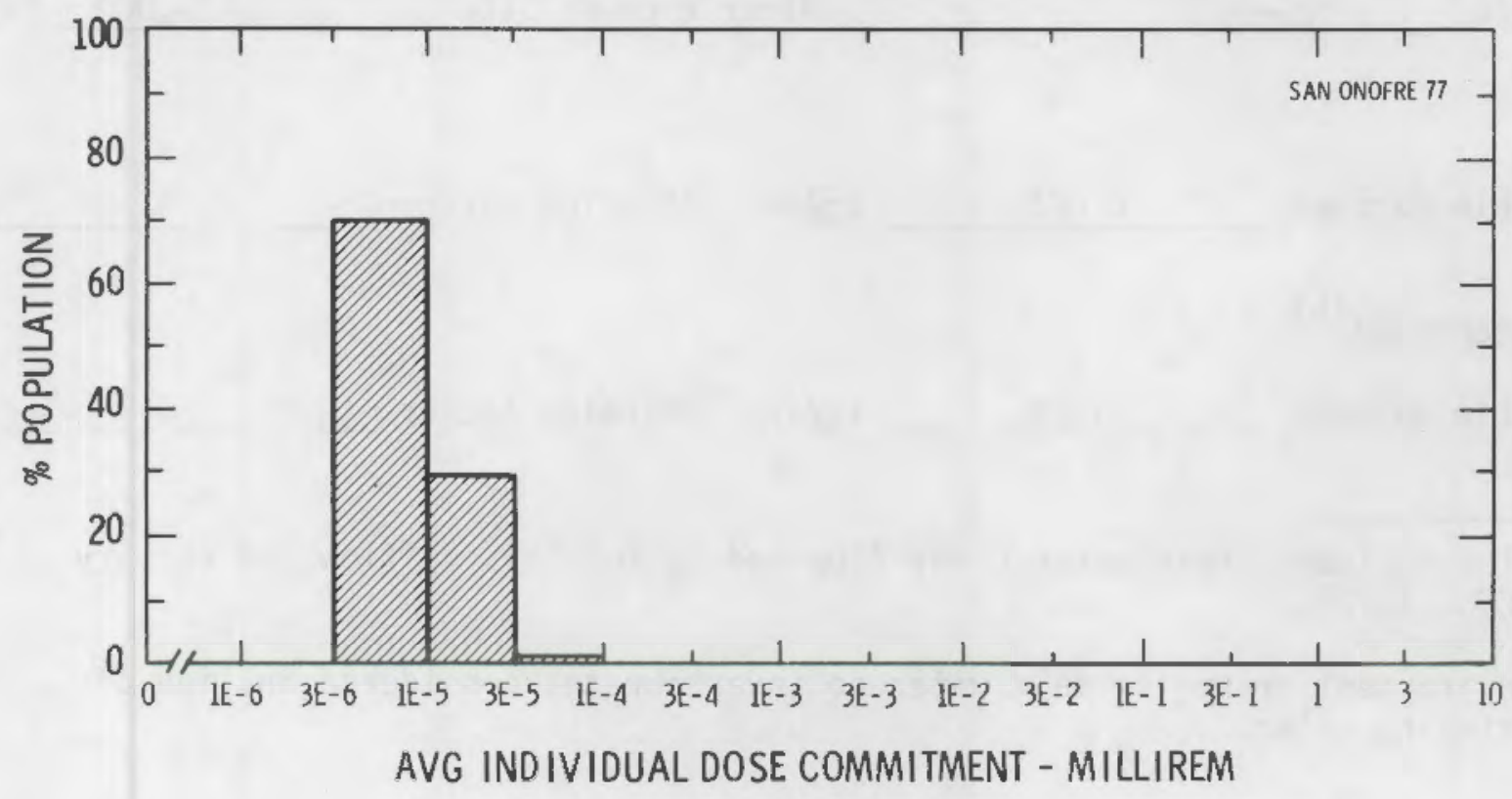


Site SURRY

Location $\mathrm{N}$

$37.1664^{\circ}$

Total Population Within 2-to-80-km Region
SURRY COUNTY, VIRGINIA

W $76.6972^{\circ}$

Major Metropolitan Centers Within Region

\begin{tabular}{l}
$\frac{\text { Center }}{\text { Newport NewS SMSA }}$ \\
\hline Norfolk SMSA \\
\hline Richmond SMSA \\
\hline
\end{tabular}

\begin{tabular}{|c|}
\hline 370,000 \\
\hline 800,000 \\
\hline 590,000 \\
\hline
\end{tabular}

\begin{tabular}{rl}
$\frac{2}{\text { Location }}$ \\
$16 \mathrm{~km}$ & ESE \\
\hline $50 \mathrm{~km}$ & $\mathrm{SE}$ \\
\hline $78 \mathrm{~km}$ & $\mathrm{NW}$ \\
\hline
\end{tabular}

Average Annual State Production of Crops and Animal Products in 80-km Radius Circle Veg $3.5 \mathrm{E} 7 \mathrm{~kg}$ Milk $1.5 \mathrm{E} 8$ \& Meat $7.4 \mathrm{E} 7 \quad \mathrm{~kg}$

Regional Productivity Factor 0.8

Animal Grazing Factor 0.7

Location of Meteorological Station Site Recovery $91 \%$

Period of Record 3 MAR $74-2$ MAR 75

Average James

River Flow at Site $25,000-\mathrm{ft}^{3} / \mathrm{s}$

Fish

Edible Harvest $6.0 \mathrm{E} 5$ $\mathrm{kg} / \mathrm{yr}$ Dilution Factor 1 Invertebrates $(b)$ Edible Harvest $1.1 \mathrm{E} 6$ $\mathrm{kg} / \mathrm{yr}$ Dilution Factor 1

(a) Flow includes fresh water river flow and saline "mixing flow" of estuary (FES, 1972).

(b) Environment primarily salt water so invertebrates considered in lieu of drinking water. 
POPULATION DOSE-COMMITMENT ESTIMATES AND

AVERAGE INDIVIDUAL DOSE-COMMITMENT HISTOGRAM FOR

SURRY 1 \& 2

Dose Commitments (person-rem) from Liquid Pathways

Inf ant

Child

Teen

Adult

TOTAL

Infant
Child
Teen
Adult
TOTAL

$\frac{G I-L L I}{0.0 E+00}$
$3.1 E-01$
$6.6 E-01$
$5.8 E+00$
$6.8 E+00$

Thyroid $0.0 \mathrm{E}+00$

2.7E+00

$1.9 \mathrm{E}+00$

$1.2 \mathrm{E}+01$

1.7E+01 \begin{tabular}{l} 
Bone \\
\hline $0.0 E+00$ \\
$3.2 E-01$ \\
$1.9 E-01$ \\
$1.1 E+00$ \\
$1.6 E+00$
\end{tabular}
Liver

$0.0 \mathrm{E}+00$

4.3E-01

3.6E-01

2. $2 \mathrm{E}+00$

$3.0 \mathrm{E}+00$

Dose Commitments (person-rem) from Airborne Pathways

\begin{tabular}{|c|c|c|c|c|c|}
\hline Total Body & GI-LLI & Thyroid & Bone & Liver & Lung \\
\hline $\begin{array}{l}1.8 \mathrm{E}-02 \\
2.2 \mathrm{E}-01 \\
1.6 \mathrm{E}-01 \\
9.3 \mathrm{E}-01\end{array}$ & $\begin{array}{l}1.8 \mathrm{E}-02 \\
2.2 \mathrm{E}-01 \\
1.6 \mathrm{E}-01 \\
9.3 \mathrm{E}-01\end{array}$ & $\begin{array}{l}8.9 \mathrm{E}-02 \\
6.7 \mathrm{E}-01 \\
3.5 \mathrm{E}-01 \\
1.6 \mathrm{E}+00\end{array}$ & $\begin{array}{l}1.2 \mathrm{E}-02 \\
1.3 \mathrm{E}-01 \\
9.3 \mathrm{E}-02 \\
5.6 \mathrm{E}-01\end{array}$ & $\begin{array}{l}1.8 \mathrm{E}-02 \\
2.3 \mathrm{E}-01 \\
1.6 \mathrm{E}-01 \\
9.3 \mathrm{E}-01\end{array}$ & $\begin{array}{l}1.9 \mathrm{E}-02 \\
2.4 \mathrm{E}-01 \\
1.8 \mathrm{E}-01 \\
1.0 \mathrm{E}+00\end{array}$ \\
\hline $1.3 E+00$ & $1.3 E+00$ & $2.7 \mathrm{E}+00$ & $7.9 E-01$ & $1.3 \mathrm{E}+00$ & $1.4 E+00$ \\
\hline
\end{tabular}

Production/consumption factors:

Produce: $<1$

Milk: $<1$

Meat: $<1$

FRACTION OF POPULATION RECEIVING AN INDICATED AVERAGE

TOTAL-BODY DOSE COMMITMENT FROM AIRBORNE PATHWAYS

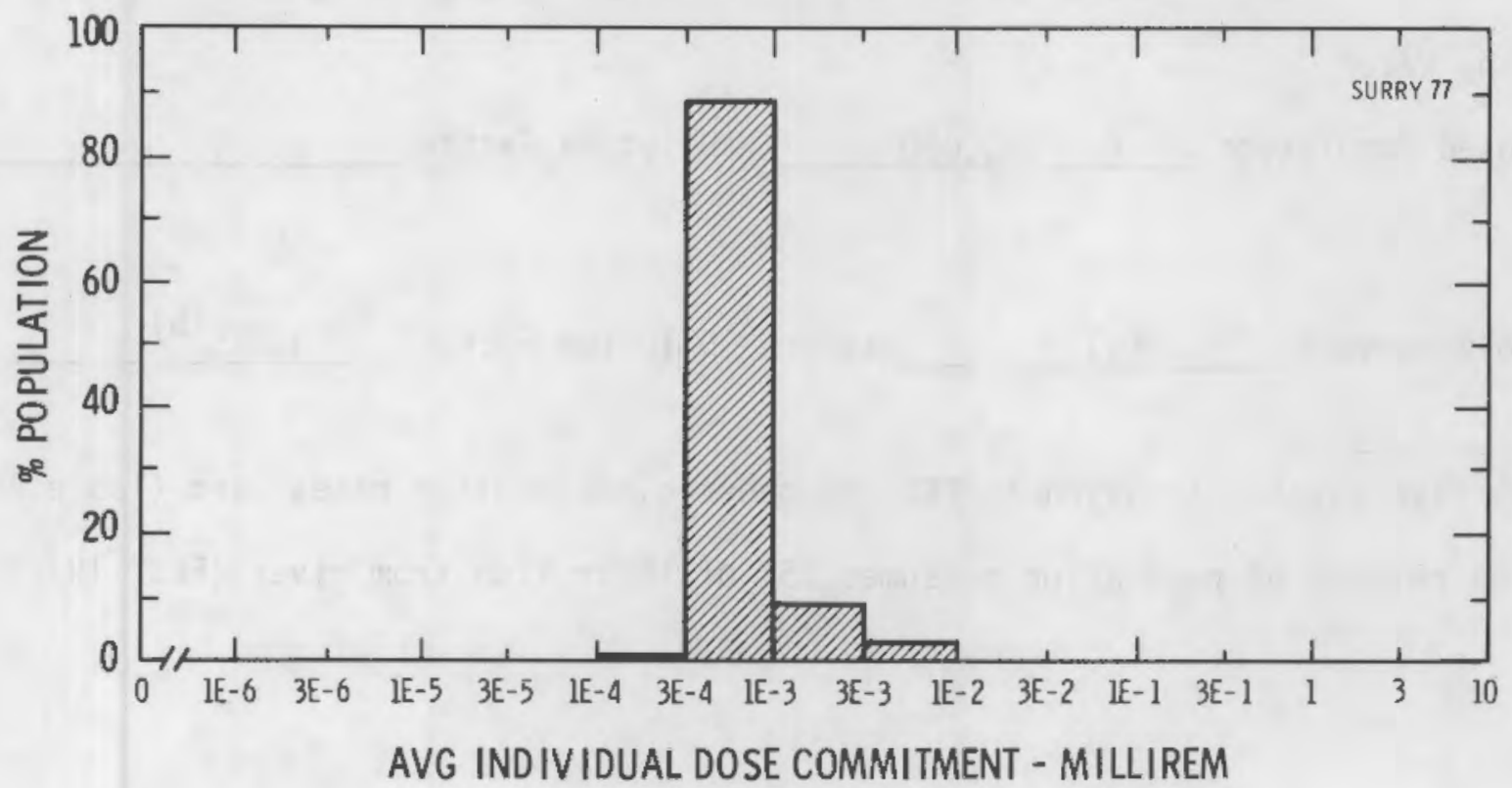


Site THREE MILE ISLAND

Location $N$ N $40.1533^{\circ}$

Total Population Within 2-to-80-km Region
THREE MILE ISLAND, PENNSYLVANIA

$76.7269^{\circ}$

$1.8 \mathrm{E} 6$

Major Metropolitan Centers Within Region

\begin{tabular}{|c|}
\hline $\begin{array}{c}\text { Center } \\
\text { Lancaster SMSA }\end{array}$ \\
\hline Harrisburg SMSA \\
\hline Reading SMSA \\
\hline York SMSA \\
\hline
\end{tabular}

\begin{tabular}{c}
$\frac{\text { Population }}{320,000}$ \\
\hline 410,000 \\
\hline 300,000 \\
\hline 330,000 \\
\hline
\end{tabular}

\begin{tabular}{lc} 
& \multicolumn{2}{l}{ Location } \\
$40 \mathrm{~km}$ & ESE \\
\hline $16 \mathrm{~km}$ & $\mathrm{NW}$ \\
\hline $71 \mathrm{~km}$ & $\mathrm{ENE}$ \\
\hline $23 \mathrm{~km}$ & $\mathrm{~S}$ \\
\hline
\end{tabular}

Average Annual State Production of Crops and Animal Products in $80-\mathrm{km}$ Radius Circle

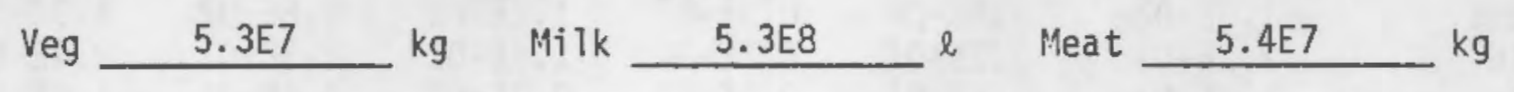

Regional Productivity Factor

1

Animal Grazing Factor

0.5

Location of Meteorological Station Site

Recovery $80 \%$

Period of Record

1 OCT $72-30$ SEP 73

Average

Susquehanna

River Flow at Site

$34,000 \mathrm{ft}^{3} / \mathrm{s}$

Drinking Water

Exposed Population

200,000

Dilution Factor

1.

Fish

Edible Harvest

(a)

$\mathrm{kg} / \mathrm{yr}$ Dilution Factor

$0.025^{(b)}$

(a) No fish catch data given in FES, so generic consumption rates used (Table A-1).

(b) Ten percent of population consumes $25 \%$ of "their fish from river (FES, 1972). 


\begin{abstract}
POPULATION DOSE-COMMITMENT ESTIMATES AND AVERAGE INDIVIDUAL DOSE-COMMITMENT HISTOGRAM FOR THREE MILE ISLAND
\end{abstract}

\begin{tabular}{|c|c|c|c|c|}
\hline Total Body & GI-LLI & Thyroid & Bone & Liver \\
\hline $\begin{array}{l}1.1 E-03 \\
2.2 E-02 \\
2.3 E-02 \\
2.4 E-01\end{array}$ & $\begin{array}{l}9.6 \mathrm{E}-04 \\
1.1 \mathrm{E}-02 \\
5.0 \mathrm{E}-03 \\
4.3 \mathrm{E}-02\end{array}$ & $\begin{array}{l}1.3 \mathrm{E}-03 \\
1.3 \mathrm{E}-02 \\
5.0 \mathrm{E}-03 \\
4.1 \mathrm{E}-02\end{array}$ & $\begin{array}{l}1.0 \mathrm{E}-03 \\
5.1 \mathrm{E}-02 \\
2.7 \mathrm{E}-02 \\
1.6 \mathrm{E}-01\end{array}$ & $\begin{array}{l}\text { 2. } 3 \mathrm{E}-03 \\
7.1 \mathrm{E}-02 \\
5.0 \mathrm{E}-02 \\
3.1 \mathrm{E}-01\end{array}$ \\
\hline $2.8 \mathrm{E}-01$ & $6.0 \mathrm{E}-02$ & $6.1 \mathrm{E}-02$ & $2.4 \mathrm{E}-01$ & 4. $3 E-01$ \\
\hline
\end{tabular}

Dose Commitments (person-rem) from Airborne Pathways

\begin{tabular}{|c|c|c|c|c|c|c|}
\hline & Total Body & GI-LLI & Thyroid & Bone & Liver & Lung \\
\hline $\begin{array}{l}\text { Infant } \\
\text { Child } \\
\text { Teen } \\
\text { Adult }\end{array}$ & $\begin{array}{l}2.4 \mathrm{E}-02 \\
2.8 \mathrm{E}-01 \\
2.0 \mathrm{E}-01 \\
1.2 \mathrm{E}+00\end{array}$ & $\begin{array}{l}2.4 \mathrm{E}-02 \\
2.8 \mathrm{E}-01 \\
2.0 \mathrm{E}-01 \\
1.2 \mathrm{E}+00\end{array}$ & $\begin{array}{l}\text { 4.6E-02 } \\
4.2 \mathrm{E}-01 \\
2.6 \mathrm{E}-01 \\
1.4 \mathrm{E}+00\end{array}$ & $\begin{array}{l}2.2 \mathrm{E}-02 \\
2.5 \mathrm{E}-01 \\
1.8 \mathrm{E}-01 \\
1.1 \mathrm{E}+00\end{array}$ & $\begin{array}{l}2.5 \mathrm{E}-02 \\
2.8 \mathrm{E}-01 \\
2.0 \mathrm{E}-01 \\
1.2 \mathrm{E}+00\end{array}$ & $\begin{array}{l}2.6 \mathrm{E}-02 \\
3.1 \mathrm{E}-01 \\
2.4 \mathrm{E}-01 \\
1.3 \mathrm{E}+00\end{array}$ \\
\hline OTAL & $1.7 E+00$ & 1. $7 E+00$ & $2.2 \mathrm{E}+00$ & $1.5 E+00$ & 1. $7 \mathrm{E}+00$ & $1.9 \mathrm{E}+00$ \\
\hline
\end{tabular}

Production/consumption factors:

Produce: $<1$

Milk: 2.2

Meat: $<1$

FRACTION OF POPULATION RECEIVING AN INDICATED AVERAGE

TOTAL-BODY DOSE COMMITMENT FROM AIRBORNE PATHWAYS

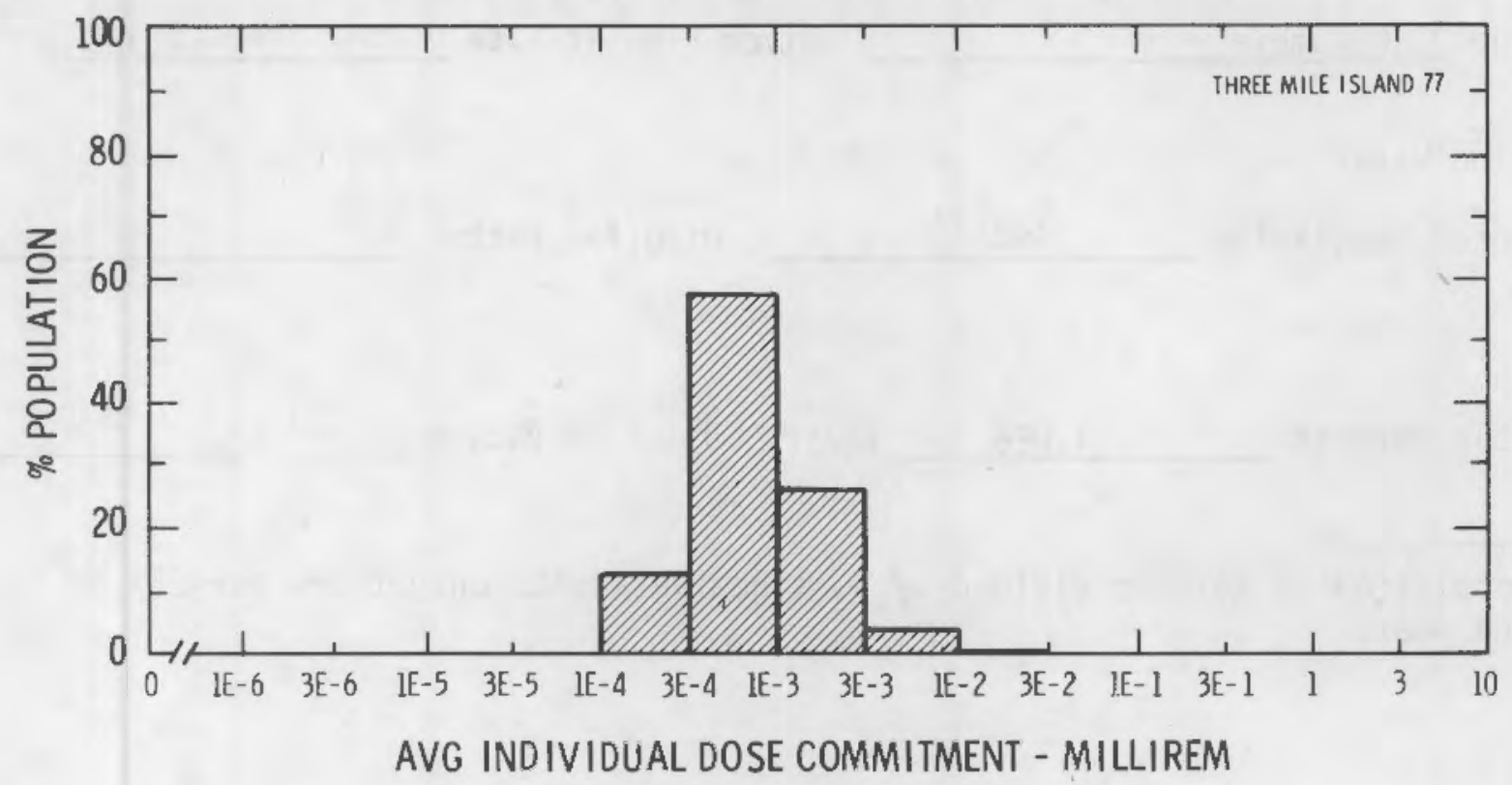


Site TROJAN

Location $\mathrm{N}$ $46.0372^{\circ}$

Total Population Within 2-to-80-km Region
PRESCOTT, OREGON

W $122.8847^{\circ}$

Major Metropolitan Centers Within Region

\begin{tabular}{l}
$\frac{1}{\text { Center }}$ \\
Portland SMSA \\
\hline Longview \\
\hline Astoria \\
\hline
\end{tabular}

\begin{tabular}{c}
$\frac{\text { Population }}{1.1 \mathrm{E} 6}$ \\
\hline 30,000 \\
\hline 11,000 \\
\hline
\end{tabular}

\begin{tabular}{ll}
\multicolumn{2}{c}{ Location } \\
$63 \mathrm{~km}$ & SSE \\
\hline $14 \mathrm{~km}$ & NNW \\
\hline $72 \mathrm{~km}$ & WNW \\
\hline
\end{tabular}

Average Annual State Production of Crops and Animal Products in $80-\mathrm{km}$ Radius Circle Veg $2.4 \mathrm{EE} 7 \mathrm{~kg} \mathrm{Milk}-3.7 \mathrm{EZ}{ }^{\ell}$ Meat $2.6 \mathrm{E} 7{ }^{\mathrm{kg}}$

Regional Productivity Factor

Animal Grazing Factor 0.9

Location of Meteorological Station Site

Period of Record 1 SEP 71 - 31 AUG 74

Average Columbia River Flow at Site $2.3 E 5 \mathrm{ft}^{3} / \mathrm{s}$ Drinking Water Exposed Population ${ }_{490}(\mathrm{a})$ Dilution Factor 1 Fish Edible Harvest $1.0 \mathrm{E} 6$ $\mathrm{kg} / \mathrm{yr}$ Dilution Factor Recovery $\quad 90 \%$ 0.75 


\begin{tabular}{|c|c|c|c|c|c|}
\hline & $\begin{array}{l}\text { POPUL } \\
\text { AVERAGE IN }\end{array}$ & $\begin{array}{l}\text { DOSE-COI } \\
\text { DUAL DOSE }\end{array}$ & $\begin{array}{l}\text { NT EST I } \\
\text { IITMENT }\end{array}$ & $\begin{array}{l}\text { AND } \\
\text { aRAM FOR }\end{array}$ & \\
\hline & Dose Commi & s (persor & ) from $L i$ & Pathways & \\
\hline & Total Body & GI-LLI & Thyroid & Bone & Liver \\
\hline $\begin{array}{l}\text { Infant } \\
\text { Child } \\
\text { Teen } \\
\text { Adult }\end{array}$ & $\begin{array}{l}8.9 \mathrm{E}-07 \\
1.4 \mathrm{E}-03 \\
2.2 \mathrm{E}-03 \\
2.2 \mathrm{E}-02\end{array}$ & $\begin{array}{l}8.2 \mathrm{E}-07 \\
1.4 \mathrm{E}-03 \\
3.0 \mathrm{E}-03 \\
2.6 \mathrm{E}-02\end{array}$ & $\begin{array}{l}1.5 \mathrm{E}-05 \\
2.7 \mathrm{E}-03 \\
1.9 \mathrm{E}-03 \\
1.2 \mathrm{E}-02\end{array}$ & $\begin{array}{l}4.4 \mathrm{E}-07 \\
6.0 \mathrm{E}-03 \\
3.6 \mathrm{E}-03 \\
2.1 \mathrm{E}-02\end{array}$ & $\begin{array}{l}8.3 E-07 \\
6.1 E-03 \\
5.1 E-03 \\
3.0 E-02\end{array}$ \\
\hline TOTAL & $2.6 \mathrm{E}-02$ & $3.1 \mathrm{E}-02$ & 1.7E-02 & $3.1 E-02$ & 4.1E-02 \\
\hline
\end{tabular}

Dose Commitments (person-rem) from Airborne Pathways

\begin{tabular}{|c|c|c|c|c|c|c|}
\hline & Total Body & GI-LLI & Thyroid & Bone & Liver & Lung \\
\hline $\begin{array}{l}\text { Infant } \\
\text { Child } \\
\text { Teen } \\
\text { Adult }\end{array}$ & $\begin{array}{l}1.2 \mathrm{E}-03 \\
1.5 \mathrm{E}-02 \\
1.0 \mathrm{E}-02 \\
6.2 \mathrm{E}-02\end{array}$ & $\begin{array}{l}1.2 \mathrm{E}-03 \\
1.3 \mathrm{E}-02 \\
9.8 \mathrm{E}-03 \\
5.9 \mathrm{E}-02\end{array}$ & $\begin{array}{l}6.4 \mathrm{E}-03 \\
6.6 \mathrm{E}-02 \\
3.4 \mathrm{E}-02 \\
1.6 \mathrm{E}-01\end{array}$ & $\begin{array}{l}1.3 \mathrm{E}-03 \\
2.1 \mathrm{E}-02 \\
1.4 \mathrm{E}-02 \\
8.0 \mathrm{E}-02\end{array}$ & $\begin{array}{l}1.2 \mathrm{E}-03 \\
1.3 \mathrm{E}-02 \\
9.7 \mathrm{E}-03 \\
5.9 \mathrm{E}-02\end{array}$ & $\begin{array}{l}1.3 \mathrm{E}-03 \\
1.5 \mathrm{E}-02 \\
1.2 \mathrm{E}-02 \\
6.6 \mathrm{E}-02\end{array}$ \\
\hline TOTAL & $8.8 \mathrm{E}-02$ & $8.3 E-02$ & 2.7E-01 & $1.2 E-01$ & 8. $3 E-02$ & $9.5 \mathrm{E}-02$ \\
\hline
\end{tabular}

Production/consumption factors:

Produce: $<1 \quad$ Milk: $<1 \quad$ Meat: <1

FRACTION OF POPULATION RECEIVING AN INDICATED AVERAGE

TOTAL-BODY DOSE COMMITMENT FROM AIRBORNE PATHWAYS

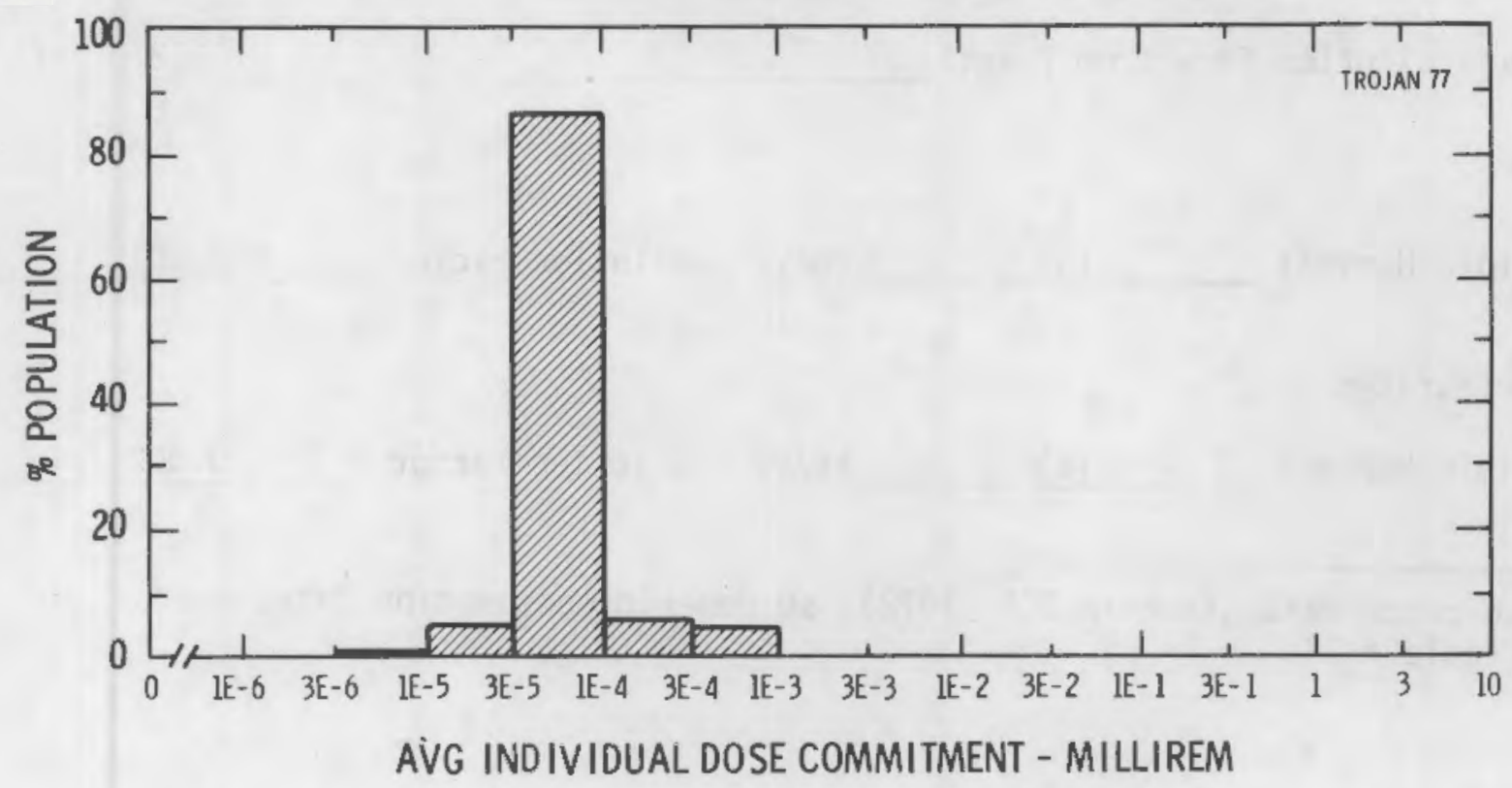


Site TURKEY POINT

DADE COUNTY, FLORIDA

Location $\mathrm{N}$

$25.4336^{\circ}$

W

$80.3317^{\circ}$

Total Population Within 2-to-80-km Region

$2.1 \mathrm{E} 6$

Major Metropolitan Centers Within Region

Center

Miami SMSA
Population

$1,500,000$
Location

$48 \mathrm{~km}$

NNE

Average Annual State Production of Crops and Arimal Products in $80-\mathrm{km}$ Radius Circle

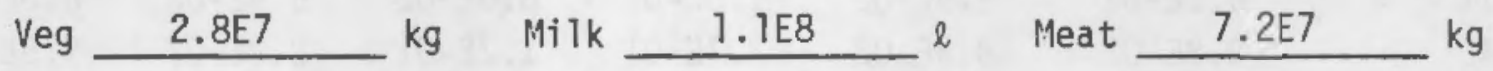

Regional Productivity Factor

$\frac{0.4}{1}$

Animal Grazing Factor

Recovery $98 \%$

Location of Meteorological Station Site

Period of Record

1 JAN $73-31$ OEC 73

Average Dilution Flow from Plant

$3,310 \mathrm{ft}^{3} / \mathrm{s}$

Fish

Edible Harvest

(a)

$\mathrm{kg} / \mathrm{yr}$ Dilution Factor

0.001

Invertebrates

Edible Harvest

(a)

$\mathrm{kg} / \mathrm{yr}$ Dilution Factor

0.002

(a) No catch data given in DES (1972), so generic consumption rates used (Table A-1). 


\section{POPULATION DOSE-COMMITMENT ESTIMATES AND AVERAGE INDIVIDUAL. DOSE-COMMITMENT HISTOGRAM FOR TURKEY POINT $3 \& 4$}

Dose Commitments (person-rem) from Liquid Pathways

Infant

Child

Teen

Adult

TOTAL

Inf ant
Child
Teen
Adult
TOTAL

$\frac{G I-L L I}{0.0 E+00}$
$4.1 E-03$
$8.5 E-03$
$7.5 E-02$
$8.7 E-02$

Thyroid

$0.0 \mathrm{E}+00$

$6.9 \mathrm{E}-02$

$4.9 \mathrm{E}-02$

$3.2 \mathrm{E}-01$

4.4E-01 $\frac{\text { Bone }}{0.0 \mathrm{E}+00}$

$3.3 \mathrm{E}-03$

2.0E -03

1.1E-02

1.7 -02
Liver

$0.0 \mathrm{E}+00$

$4.2 \mathrm{E}-03$

$3.6 \mathrm{E}-03$

2.1E-02

2. $9 \mathrm{E}-02$

Dose Commitments (person-rem) from Airborne Pathways

\begin{tabular}{|c|c|c|c|c|c|}
\hline Total Body & GI-LLI & Thyroid & Bone & Liver & Lung \\
\hline $\begin{array}{l}5.9 \mathrm{E}-03 \\
6.3 \mathrm{E}-02 \\
4.5 \mathrm{E}-02 \\
2.7 \mathrm{E}-01\end{array}$ & $\begin{array}{l}5.3 E-03 \\
5.9 E-02 \\
4.4 E-02 \\
2.7 E-01\end{array}$ & $\begin{array}{l}5.0 \mathrm{E}-01 \\
2.8 \mathrm{E}+00 \\
1.1 \mathrm{E}+00 \\
3.7 \mathrm{E}+00\end{array}$ & $\begin{array}{l}6.6 \mathrm{E}-03 \\
6.7 \mathrm{E}-02 \\
4.5 \mathrm{E}-02 \\
2.7 \mathrm{E}-01\end{array}$ & $\begin{array}{l}6.8 \mathrm{E}-03 \\
6.7 \mathrm{E}-02 \\
4.7 \mathrm{E}-02 \\
2.7 \mathrm{E}-01\end{array}$ & $\begin{array}{l}5.7 \mathrm{E}-03 \\
6.6 \mathrm{E}-02 \\
5.1 \mathrm{E}-02 \\
2.9 \mathrm{E}-01\end{array}$ \\
\hline 3. $8 \mathrm{E}-01$ & 3. $7 \mathrm{E}-01$ & $8.1 \mathrm{E}+00$ & $3.8 E-01$ & $3.9 \mathrm{E}-01$ & $4.1 \mathrm{E}-01$ \\
\hline
\end{tabular}

Production/consumption factors:

Produce: $<1$

Milk: $<1$

Meat: $<1$

FRACTION OF POPULATION RECEIVING AN INDICATED AVERAGE TOTAL-BODY DOSE COMMITMENT FROM AIRBORNE PATHWAYS

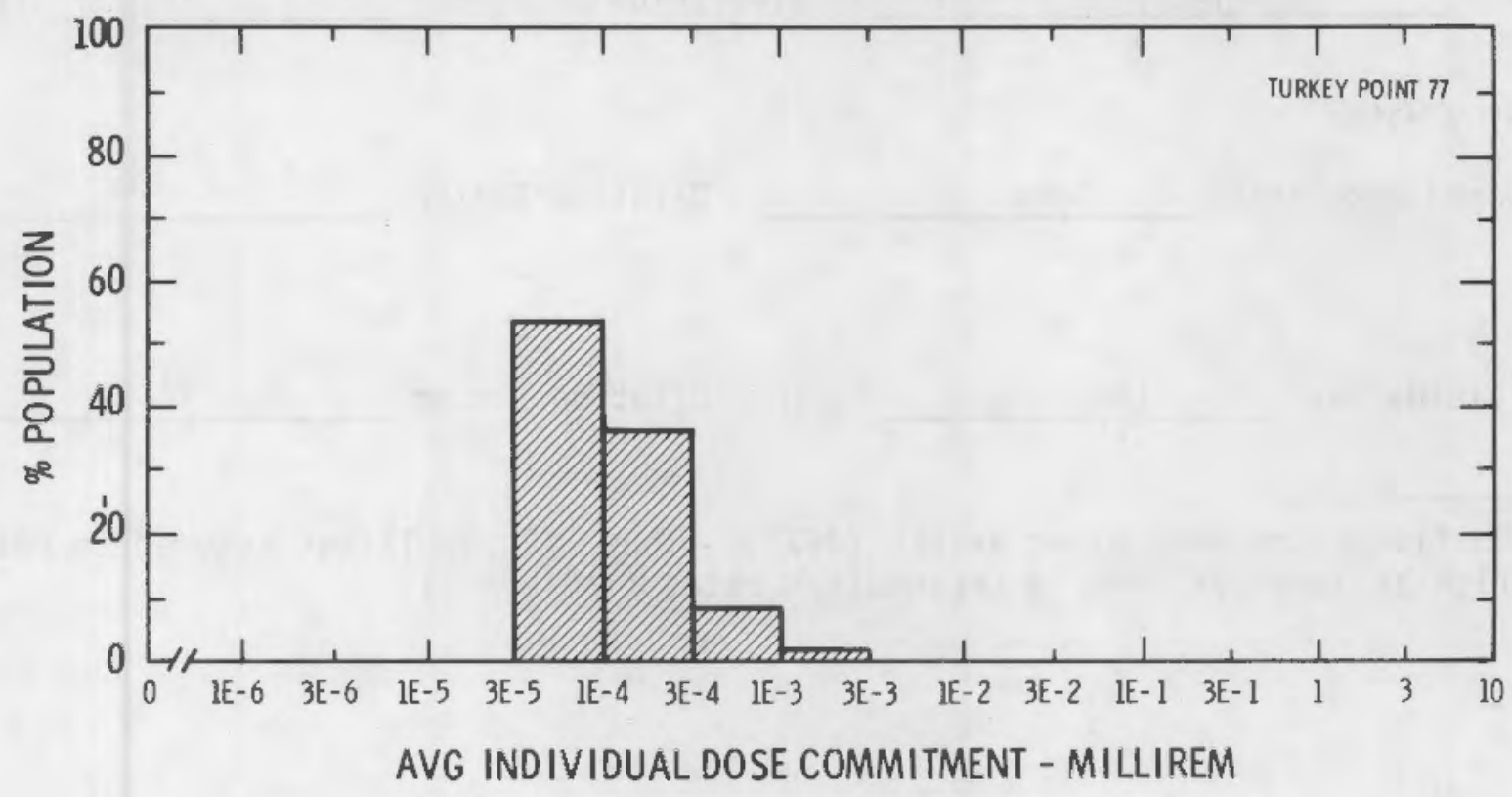


Site VERMONT YANKEE

Location $\mathrm{N}$ $42.7803^{\circ}$

Tota 1 Population Within 2-to-80-km Region
VERNON, VERMONT

W $72.5158^{\circ}$

Major Metropolitan Centers Within Region

\begin{tabular}{l} 
Center \\
Pittsfield \\
\hline Springfield SMSA \\
\hline Worcester SMSA \\
\hline
\end{tabular}

$\begin{array}{r}\frac{\text { Population }}{58,000} \\ \hline 620,000 \\ \hline 680,000 \\ \hline\end{array}$

\begin{tabular}{ll}
$70 \mathrm{~km}$ & $\mathrm{SW}$ \\
\hline $72 \mathrm{~km}$ & $\mathrm{~S}$ \\
\hline $80 \mathrm{~km}$ & $\mathrm{SE}$ \\
\hline
\end{tabular}

Average Annual State Production of Crops and Animal Products in $80-\mathrm{km}$ Radius Circle

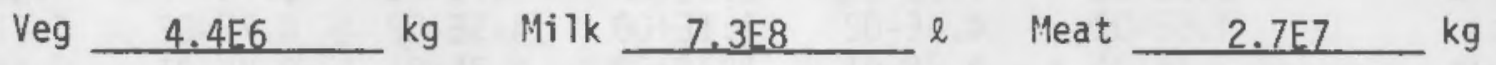

Regional Productivity Factor

Animal Grazing Factor

$\frac{1}{0.4}$

Location of Meteorological Station

Site

Recovery $97 \%$

Period of Record 1 APR 75 - 31 MAR 76

Average Connecticut

River Flow at Site $10,000 \mathrm{ft}^{3} / \mathrm{s}$

Drinking Water

Exposed Population None Dilution Factor

Fish

Edible Harvest (a) $\mathrm{kg} / \mathrm{yr}$ Dilution Factor $0.5^{(a)}$

(a) No fish catch data given in FES (1972). Thus $1 / 2$ population assumed to eat fish at level of generic consumption rates (Table A-1). 
Infant

Child

Teen

Adult

TOTAL
Infant

Child

Teen

Adult

TOTAL

\begin{abstract}
POPULATION DOSE-COMMITMENT ESTIMATES AND
AVERAGE INDIVIDUAL DOSE-COMMITMENT HISTOGRAM FOR

VERMONT YANKEE
\end{abstract}

Dose Commitments (person-rem) from Liquid Pathways

\begin{tabular}{|c|c|c|c|c|}
\hline Total Body & GI-LLI & Thyroid & Bone & Liver \\
\hline $\begin{array}{l}0.0 \mathrm{E}+00 \\
1.1 \mathrm{E}-02 \\
2.0 \mathrm{E}-02 \\
2.1 \mathrm{E}-01\end{array}$ & $\begin{array}{l}0.0 \mathrm{E}+00 \\
1.7 \mathrm{E}-03 \\
3.5 \mathrm{E}-03 \\
3.0 \mathrm{E}-02\end{array}$ & $\begin{array}{l}0.0 \mathrm{E}+00 \\
1.8 \mathrm{E}-03 \\
1.3 \mathrm{E}-03 \\
8.5 \mathrm{E}-03\end{array}$ & $\begin{array}{l}0.0 \mathrm{E}+00 \\
5.6 \mathrm{E}-02 \\
3.4 \mathrm{E}-02 \\
2.0 \mathrm{E}-01\end{array}$ & $\begin{array}{l}0.0 \mathrm{E}+00 \\
6.0 \mathrm{E}-02 \\
5.0 \mathrm{E}-02 \\
3.0 \mathrm{E}-01\end{array}$ \\
\hline $2.4 \mathrm{E}-01$ & $3.5 E-02$ & $1.2 E-02$ & $2.9 \mathrm{E}-01$ & 4.1E-01 \\
\hline
\end{tabular}

Dose Commitments (person-rem) from Airborne Pathways

Production/consumption factors:

Produce: $<1 \quad$ Milk: 4.0 Meat: <1

FRACTION OF POPULATION RECEIVING AN INDICATED AVERAGE

TOTAL-BODY DOSE COMMITMENT FROM AIRBORNE PATHWAYS

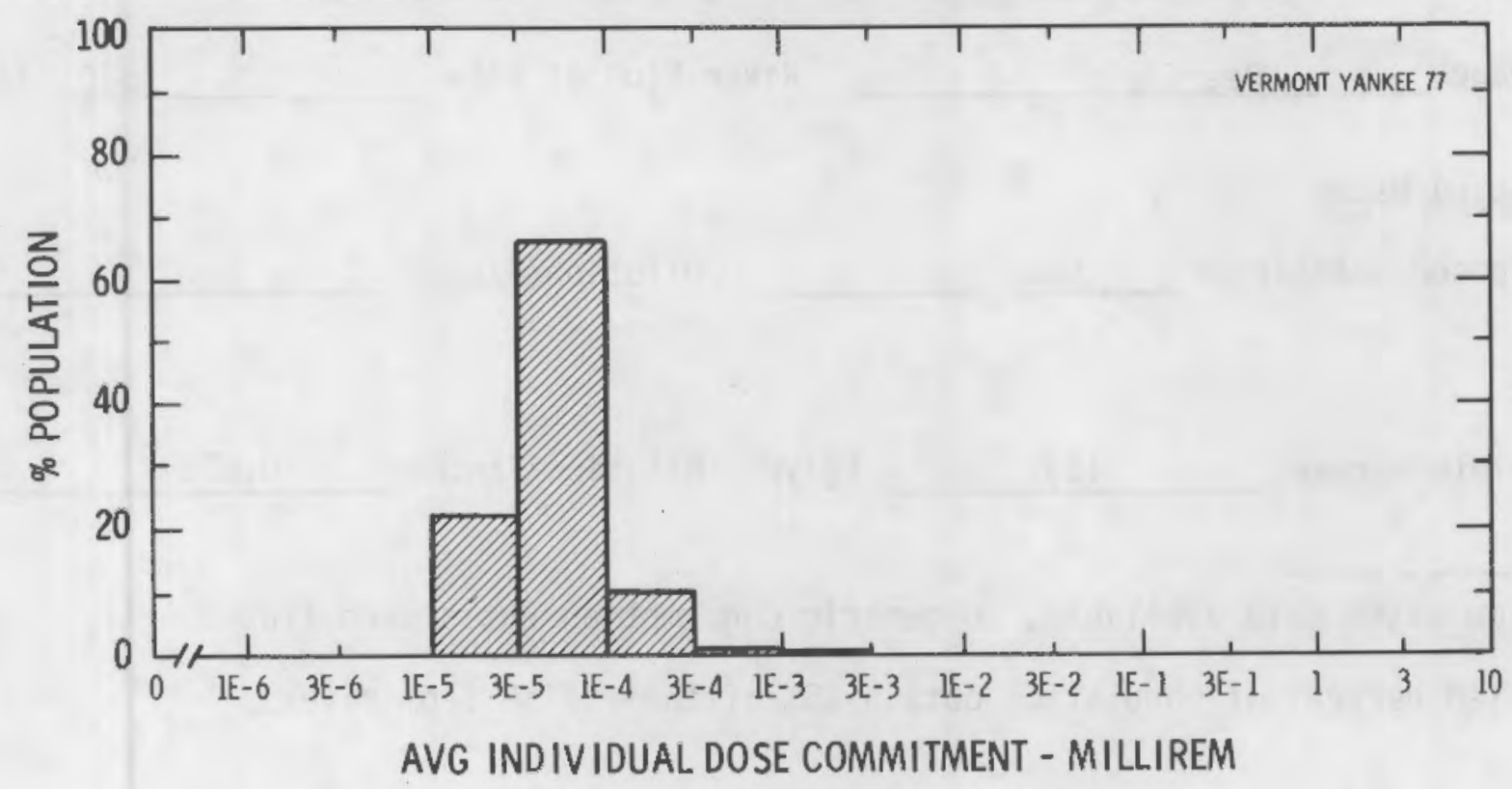


Site YANKEE ROWE

ROWE, MASSACHUSETTS

Location $\mathrm{N}$ $42.7287^{\circ}$

$72.9247^{\circ}$

Total Population Within 2-to-80-km Region $1.6 \mathrm{E} 6$

Major Metropolitan Centers Within Region

$\frac{\frac{\text { Center }}{\text { Springfield SMSA }}}{\text { Pittsfield }}$

$\frac{\frac{\text { Population }}{600,000}}{58,000}$

\begin{tabular}{cc}
$\frac{2}{2}$ Location \\
$64 \mathrm{~km}$ & SSE \\
\hline $34 \mathrm{~km}$ & SW \\
\hline $72 \mathrm{~km}$ & $\mathrm{~W}$ \\
\hline
\end{tabular}

Average Annual State Production of Crops and Animal Products in $80-\mathrm{km}$ Radius Circie Veg $2.0 \mathrm{E} 7 \quad \mathrm{~kg} M \mathrm{Milk}-2.6 \mathrm{E} 8{ }^{\ell}$ Meat $1.6 \mathrm{EF} 7 \mathrm{~kg}$

Regional Productivity Factor

Animal Grazing Factor

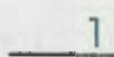

Location of Meteorological Station

Site

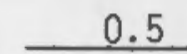

Period of Record 1 OCT $71-30$ SEP 72

Average Deerfield

River Flow at Site $570 \mathrm{ft}^{3} / \mathrm{s}$

Drinking Water

Exposed Population None

Dilution Factor

Fish

Edible Harvest

(a) $\mathrm{kg} / \mathrm{yr}$ Dilution Factor $0.025^{(b)}$

(a) No catch data available, so generic consumption rates used (Table A-1).

(b) Ten percent of population obtain $25 \%$ of their fish from river. 


\section{POPULATION DOSE-COMMITMENT ESTIMATES AND AVERAGE INDIVIDUAL DOSE-COMMITMENT HISTOGRAM FOR YANKEE ROWE}

Dose Commitments (person-rem) from Liquid Pathways

\begin{tabular}{|c|c|c|c|c|c|}
\hline & Total Body & GI-LLI & Thyroid & Bone & Liver \\
\hline $\begin{array}{l}\text { Inf ant } \\
\text { Child } \\
\text { Teen } \\
\text { Adult }\end{array}$ & $\begin{array}{l}0.0 \mathrm{E}+00 \\
1.6 \mathrm{E}-02 \\
1.7 \mathrm{E}-02 \\
1.5 \mathrm{E}-01\end{array}$ & $\begin{array}{l}0.0 \mathrm{E}+00 \\
1.2 \mathrm{E}-02 \\
9.2 \mathrm{E}-03 \\
6.3 \mathrm{E}-02\end{array}$ & $\begin{array}{l}0.0 \mathrm{E}+00 \\
2.1 \mathrm{E}-02 \\
1.4 \mathrm{E}-02 \\
9.2 \mathrm{E}-02\end{array}$ & $\begin{array}{l}0.0 \mathrm{E}+00 \\
7.1 \mathrm{E}-02 \\
4.2 \mathrm{E}-02 \\
2.4 \mathrm{E}-01\end{array}$ & $\begin{array}{l}0.0 \mathrm{E}+00 \\
4.0 \mathrm{E}-02 \\
3.2 \mathrm{E}-02 \\
1.9 \mathrm{E}-01\end{array}$ \\
\hline TOTAL & $1.8 E-01$ & $8.4 E-02$ & 1. $3 E-01$ & $3.5 E-01$ & $2.6 \mathrm{E}-01$ \\
\hline
\end{tabular}

Dose Commitments (person-rem) from Airborne Pathways

\begin{tabular}{|c|c|c|c|c|c|c|}
\hline & Total Body & GI-LLI & Thyroid & Bone & Liver & Lung \\
\hline $\begin{array}{l}\text { Inf ant } \\
\text { Child } \\
\text { Teen } \\
\text { Adult }\end{array}$ & $\begin{array}{l}6.9 E-04 \\
5.0 E-03 \\
2.0 E-03 \\
8.6 E-03\end{array}$ & $\begin{array}{l}6.9 \mathrm{E}-04 \\
5.0 \mathrm{E}-03 \\
2.0 \mathrm{E}-03 \\
8.6 \mathrm{E}-03\end{array}$ & $\begin{array}{l}7.3 \mathrm{E}-04 \\
5.3 \mathrm{E}-03 \\
2.2 \mathrm{E}-03 \\
9.0 \mathrm{E}-03\end{array}$ & $\begin{array}{l}2.7 \mathrm{E}-03 \\
1.9 \mathrm{E}-02 \\
6.2 \mathrm{E}-03 \\
2.0 \mathrm{E}-02\end{array}$ & $\begin{array}{l}6.9 \mathrm{E}-04 \\
5.0 \mathrm{E}-03 \\
2.0 \mathrm{E}-03 \\
8.6 \mathrm{E}-03\end{array}$ & $\begin{array}{l}6.9 \mathrm{E}-04 \\
5.1 \mathrm{E}-03 \\
2.2 \mathrm{E}-03 \\
9.0 \mathrm{E}-03\end{array}$ \\
\hline TOTAL & $1.6 E-02$ & $1.6 E-02$ & $1.7 \mathrm{E}-02$ & $4.8 E-02$ & $1.6 \mathrm{E}-02$ & 1.7E-02 \\
\hline
\end{tabular}

Production/consumption factors:

Produce: < Milk: 1.3 Meat: <

FRACTION OF POPULATION RECEIVING AN INDICATED AVERAGE

TOTAL-BODY DOSE COMMITMENT FROM AIRBORNE PATHWAYS

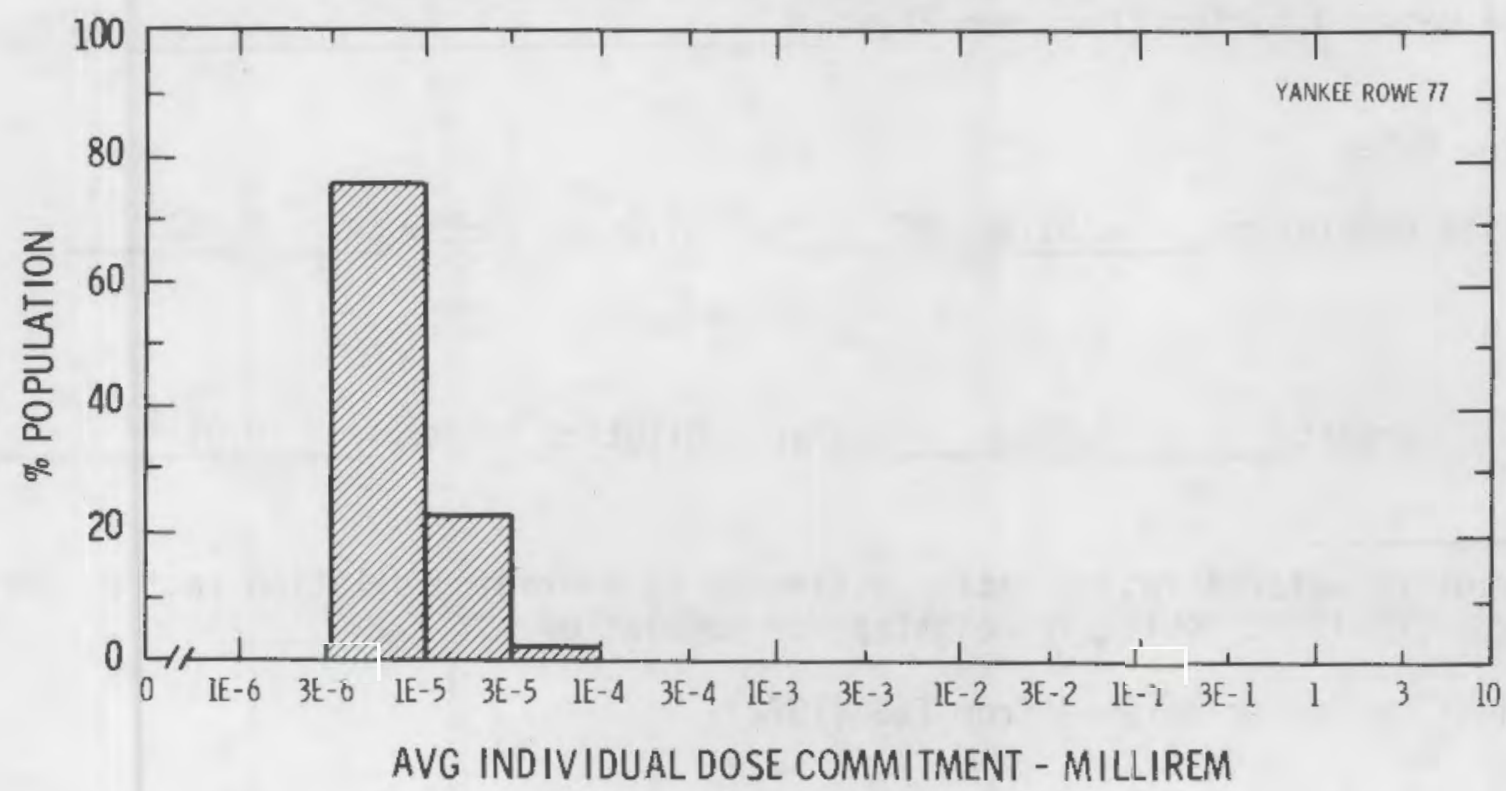


Site ZION

Location $\mathrm{N}$

$42.4594^{\circ}$

Total Population Within 2-to-80-km Region
ZION, ILLINOIS

W $87.8067^{\circ}$

$7.0 \mathrm{E} 6$

Major Metropolitan Centers Within Region

\begin{tabular}{ll}
$\frac{\text { Center }}{\text { Chicago SMSA }(2 / 3)}$ \\
$\frac{\text { Milwaukee }}{\text { Kenosha }} \frac{\frac{\text { Population }}{4,600,000}}{\frac{1,400,000}{80,000}} \frac{\frac{64 \mathrm{~km}}{\text { Location }}}{\frac{64 \mathrm{~km}}{14 \mathrm{~km}} \mathrm{NSE}}$ \\
\hline
\end{tabular}

Average Annual State Production of Crops and Animal Products in $80-\mathrm{km}$ Radius Circle Veg $1.1 \mathrm{E} 8$ _ $\mathrm{kg}$ Milk $1.8 \mathrm{E} 8{ }^{\ell}$ Meat $1.9 \mathrm{E} 8{ }^{\mathrm{kg}}$

Regional Productivity Factor

0.5

Animal Grazing Factor

0.5

Location of Meteorological Station

Site

Recovery $88 \%$

Period of Record 1 JAN $74-31$ DEC 75

Average Water Dilution Flow from Plant $1100 \mathrm{ft}^{3} / \mathrm{s}$

Drinking Water

Exposed Population

$6,700,000$

Dilution Factor

$0.037^{(a)}$

Fish

Edible Harvest $5.0 \mathrm{E} 6$ $\mathrm{kg} / \mathrm{yr}$ Dilution Factor $0.01^{(b)}$

(a) Drinking water dilution factor estimated by averaging dilution factors derived from FES (1972) suitably weighted for population.

(b) Dilution factor derived from FES (1972). 


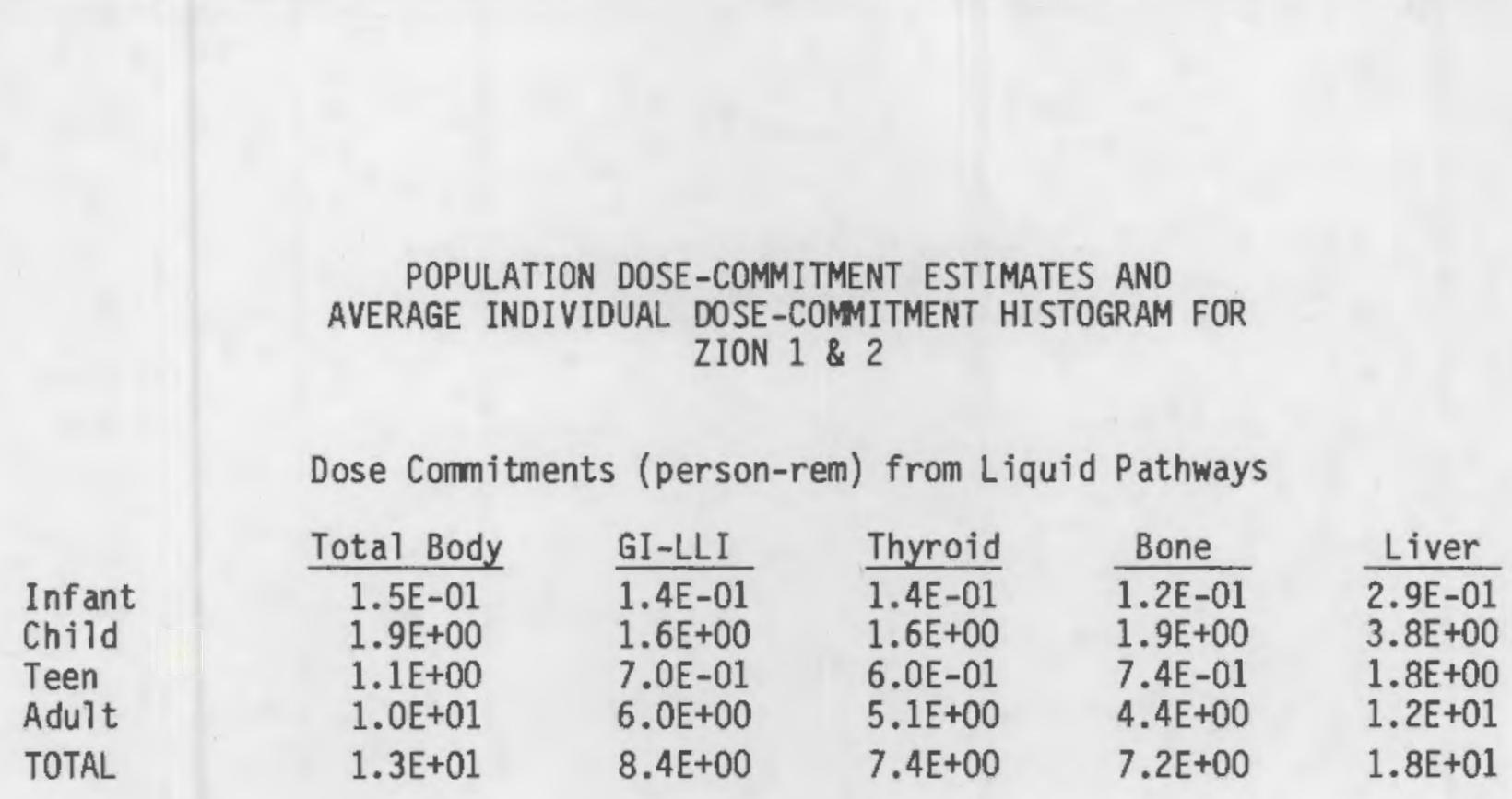

Dose Comitments (person-rem) from Airborne Pathways

\begin{tabular}{|c|c|c|c|c|c|c|}
\hline & Total Body & GI-LLI & Thyroid & Bone & Liver & Lung \\
\hline $\begin{array}{l}\text { Inf ant } \\
\text { Child } \\
\text { Teen } \\
\text { Adult }\end{array}$ & $\begin{array}{l}1.4 E-01 \\
1.5 E+00 \\
1.1 E+00 \\
6.7 E+00\end{array}$ & $\begin{array}{l}1.3 E-01 \\
1.5 E+00 \\
1.1 E+00 \\
6.7 E+00\end{array}$ & $\begin{array}{l}1.5 \mathrm{E}-01 \\
1.6 \mathrm{E}+00 \\
1.2 \mathrm{E}+00 \\
6.9 \mathrm{E}+00\end{array}$ & $\begin{array}{l}1.4 \mathrm{E}-01 \\
1.6 \mathrm{E}+00 \\
1.1 \mathrm{E}+00 \\
6.7 \mathrm{E}+00\end{array}$ & $\begin{array}{l}1.4 \mathrm{E}-01 \\
1.6 \mathrm{E}+00 \\
1.1 \mathrm{E}+00 \\
6.7 \mathrm{E}+00\end{array}$ & $\begin{array}{l}1.4 \mathrm{E}-01 \\
1.6 \mathrm{E}+00 \\
1.2 \mathrm{E}+00 \\
7.2 \mathrm{E}+00\end{array}$ \\
\hline TOTAL & $9.5 E+00$ & $9.4 E+00$ & $9.8 E+00$ & $9.5 \mathrm{E}+00$ & $9.6 \mathrm{E}+00$ & $1.0 E+01$ \\
\hline
\end{tabular}

Production/consumption factors:

$\begin{array}{lll}\text { Produce: }<1 & \text { Milk: }<1 & \text { Meat: }<1\end{array}$

FRACTION OF POPULATION RECEIVING AN INDICATED AVERAGE

TOTAL-BODY DOSE COMMITMENT FROM AIRBORNE PATHWAYS

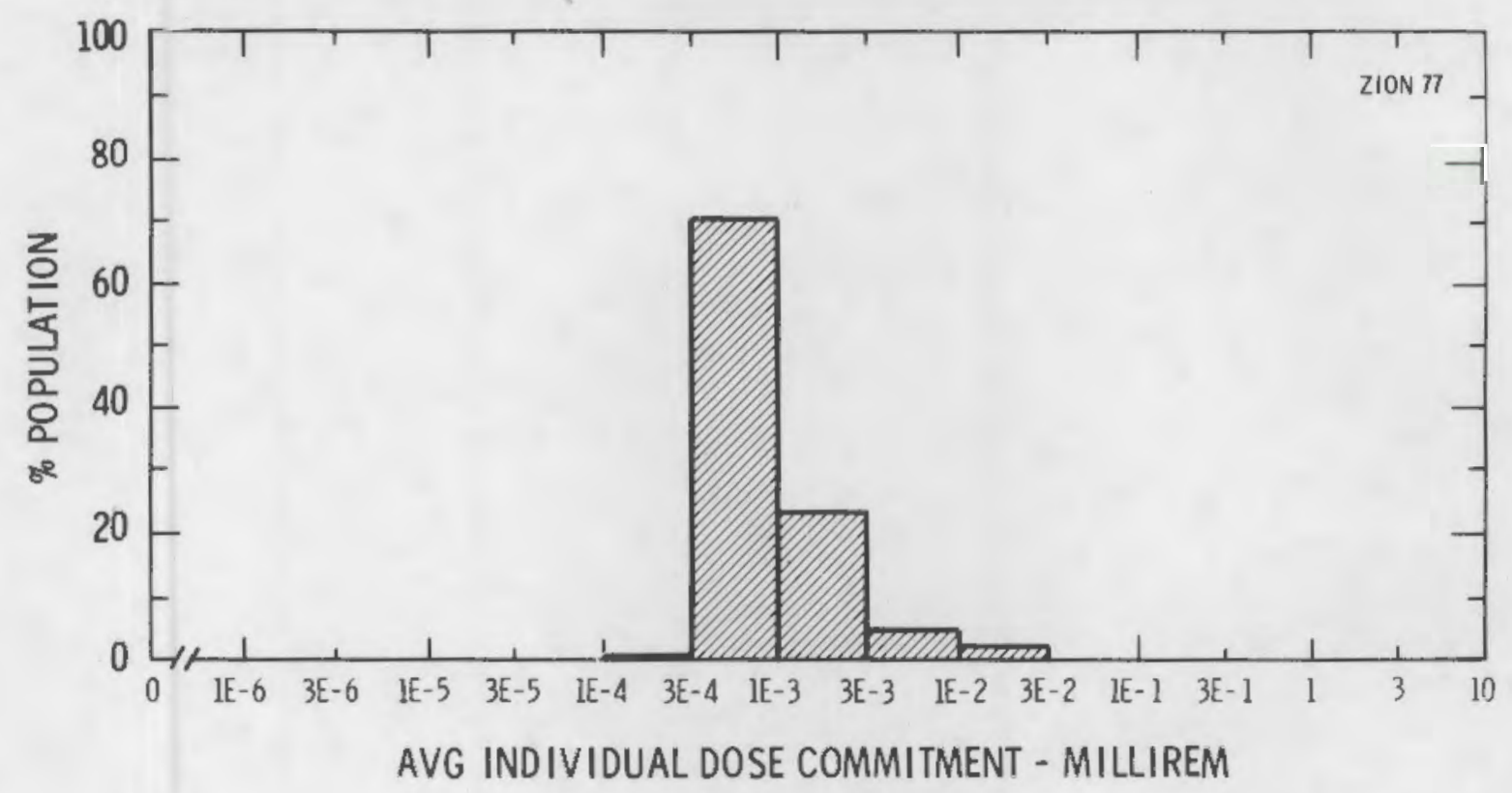





\section{REFERENCES}

Baker, D. A., J. K. Soldat and E. C. Watson. 1977. Population Dose Commitments Due to Radioactive Releases from Nuclear Power Plant Sites in 1975, PNL-2439. Pacific Northwest Laboratory, Richland, WA.

Baker, D. A. 1979. Population Dose Commitments Due to Radioactive Releases from Nuclear Power Plant Sites in 1976, NUREG/CR-1125, PNL-2940. U.S. Nuc lear Regulatory Commission, Washington, DC. *

Decker, T. R. 1979. Radioactive Materials Released from Nuclear Power Plants (1977), NUREG-0521. U.S. Nuc Tear Regulatory Commission, Washington, DC. *

Final Environmental Statement Concerning Proposed Rule-Making Action: Numerical Guides for Design Objectives and Limiting Conditions for Operation to Meet the Criterion "As Low As Practicable" for Radioactive Material in Light-Water-Cooled Nuclear Power Reactor Effluents. 1973. WASH-1258, Vo1. 1. Directorate of Regulatory Standards, U.S. Atomic Energy Commission, Washington, DC.

Noshkin, V. E., W. L. Robison and F. L. Harrison. 1976. Radiological Dose to Man Through the Marine Pathway from Reactor Operations at Humboldt Bay, California, ÜCRL-52160. Lawrence Livermore Laboratory, Livermore, $\overline{C A}$.

ॠAvailable for purchase from the NRC/GPO Sales Program, U.S. Nuclear Regulatory Commission, Washington, $D C$ 20555, and the National Technical Information Service, Springfield, VA 22161. 

APPENDIX 
APPENOIX

\section{MODELS}

The calculationa? models used were primarily those given in the Nuclear Regulatory Commission's Regulatory Guide 1.109 (1977). Computer programs were written to use these models to generate population dose commitments for four age groups. The percentages of the population comprising the four age groups were $1.44 \%$, infant; $16.0 \%$, child; $11.7 \%$, teen-ager; and $70.9 \%$, adu?t (Population Estimates and Projections, 1975). Where possible, the site-dependent parameters were taken from the environmental statements issued for each reactor (Table 3). The generic parameters used for this study such as consumption rates, occupancy factors and holdup times are given in Table A-1 and A-2 below. It should be noted that generic consumption rates for aquatic foods and inhalation rates are taken from Regulatory Guide 1.109 (1977). Bioaccumulation factors and terrestrial food transfer factors were taken from Regulatory Guide 1.109 (1977). Dose comitment factors for the four age groups were taken from Hoenes and Soldat (1977).

TABLE A-1. Generic Consumption Rates and Occupancy Factors Used for the Study of Average Members of the Population(a)

\begin{tabular}{|c|c|c|c|c|}
\hline Pathway & Infant & Child & Teen-ager & Adult \\
\hline $\begin{array}{l}\text { Fruits, vegetables and } \\
\text { grain }(\mathrm{kg} / \mathrm{yr})\end{array}$ & 0 & 200 & 240 & 190 \\
\hline Milk $(2 / \mathrm{yr})$ & 170 & 170 & 200 & 110 \\
\hline Meat and pou 7 try $(\mathrm{kg} / \mathrm{yr})$ & 0 & 37 & 59 & 95 \\
\hline Fish $(\mathrm{kg} / \mathrm{yr})(\mathrm{b})$ & 0 & 2.2 & 5.2 & 6.9 \\
\hline Invertebrates $(\mathrm{kg} / \mathrm{yr})$ & 0 & 0.33 & 0.75 & 1.0 \\
\hline Drinking water $(\ell / \mathrm{l} r)$ & $170(c)$ & 260 & 260 & 370 \\
\hline Inhalation $\left(\mathrm{m}^{3} / \mathrm{yr}\right)$ & $1400(d)$ & 3700 & 8000 & 8000 \\
\hline $\begin{array}{l}\text { Air submersion and } \\
\text { ground irradiation } \\
\text { occupancy factor }\end{array}$ & 0.5 & 0.5 & 0.5 & 0.5 \\
\hline
\end{tabular}

(a) Regulatory Guide 1.109 (1977)

(b) Both fresh- and saltwater

(c) Assumed to be equal to milk consumption

(d) Same as for maximum individual 
TABLE A-2. Holdup Times Between Harvest and Consumption of Foods(a)

Food

Fruits, grains and vegetables Milk (b) Meat (b)

Aquatic foods ( $f$ ish and invertebrates) Drinking water
Holdup Time (days)

14

4

20

7

(a) Regulatory Guide 1.109 (1977)

(b) Value given is time after milking or slaughter. For the portion of the time animals were fed stored feed, an additional 90 days was added to the holdup time.

\section{RELEASES}

The doses were estimated using the measured releases as reported by the site operators for 1977 (Decker, 1979). (a) These releases include all radionuclides specified by the NRC to be measured and reported by the operators of all commercial nuclear power plants. Radionuclides given as a combination of parent-daughter isotopes such as $\mathrm{Y} / \mathrm{Sr}-90, \mathrm{Zr} / \mathrm{Nb}-95, \mathrm{Ba} / \mathrm{La}-140$, $\mathrm{I} / \mathrm{Xe}-133$ and $\mathrm{Pr} / \mathrm{Ce}-144$ were divided eventy between the parent and daughter.

The radionuclides used in this study, aiong with their half-lives, are given in Table A-3. Note that the " $+D$ " after some of the nuclides indicates that the decay energy of the daughter is included with the parent. Thus, whenever a parent nuclide release is specified, the result of the dose calculation will be as though an additional equilibrium amount of the daughter nuclide is specified. The daughter nuclide itself will be included separately if it can be released independently of the parent and/or if it has a relatively long half-life.

\section{METEORDLDGY}

When more than one set of meteorological (joint frequency) data were available for a site, the one which appeared to be the most reliable was used to generate atmospheric transport factors. Factors were calculated for 16 compass points, and ten radii from 2 to $80 \mathrm{~km}$ (see TabTe A-4) using the NRC computer program XOQDOQ (Sagendorf 1977).

(a) Very short-1ived isotopes such as $\mathrm{Kr}-90,91,93,94, \mathrm{Xe}-139,140,141,143$ and $\mathrm{Rb}-88 \mathrm{M}$; those not likely to be produced; and those which were daughters whose decay energies were accounted for in the dose factor for the parent were not included in the dose. 
TABLE A-3. Radionuclides Considered in This Study

\begin{tabular}{|c|c|c|c|c|c|}
\hline No. & Nuclide & $\begin{array}{c}\text { Decay Constant } \\
(1 / \mathrm{sec}) \\
\end{array}$ & No. & Nuclide & $\begin{array}{c}\text { Decay Constant } \\
(1 / \text { sec }) \\
\end{array}$ \\
\hline 1 & $\mathrm{H}-3$ & $1.78 \mathrm{E}-09$ & 43 & $\mathrm{Nb}-97$ & $1.57 \mathrm{E}-04$ \\
\hline 2 & $\mathrm{Be}-10$ & $1.37 \mathrm{E}-14$ & 44 & Mo- $99+D$ & $2.92 \mathrm{E}-06$ \\
\hline 3 & $C-14$ & $3.83 \mathrm{E}-12$ & 45 & TC-99M & $3.19 \mathrm{E}-05$ \\
\hline 4 & $\mathrm{~N}-13$ & $1.16 \mathrm{E}-03$ & 46 & Ru-103+D & $2.02 \mathrm{E}-07$ \\
\hline 5 & $\mathrm{~F}-18$ & $1.05 \mathrm{E}-04$ & 47 & $R u-106+D$ & $2.17 \mathrm{E}-08$ \\
\hline 6 & $\mathrm{Na}-22$ & $8.44 \mathrm{E}-09$ & 48 & $\mathrm{Aq}-110 \mathrm{M}+\mathrm{D}$ & $3.19 \mathrm{E}-08$ \\
\hline 7 & $\mathrm{Na}-24$ & $1.28 \mathrm{E}-05$ & 49 & Cd-115M & $1.80 \mathrm{~F}-07$ \\
\hline 8 & $\mathrm{Ar}-41$ & $1.05 E-04$ & 50 & Cd-115 & $3.60 \mathrm{E}-06$ \\
\hline 9 & Sc -46 & $9.58 \mathrm{E}-08$ & 51 & $S n-125+D$ & $8.31 E-07$ \\
\hline 10 & $C r-51$ & $2.89 E-07$ & 52 & Sb-124 & $1.33 \mathrm{E}-07$ \\
\hline 11 & $M n-54$ & $2.57 \mathrm{E}-08$ & 53 & Sb $-125+D$ & $8.06 \mathrm{E}-09$ \\
\hline 12 & $M n-56$ & $7.47 \mathrm{E}-05$ & 54 & Te-132+D & $2.47 \mathrm{E}-06$ \\
\hline 13 & $\mathrm{Fe}-55$ & $8.14 E-09$ & 55 & $T e-133 M+D$ & $2.09 \mathrm{E}-04$ \\
\hline 14 & $\mathrm{Fe}-59$ & $1.80 \mathrm{E}-07$ & 56 & $I-131+D$ & $9.97 \mathrm{E}-07$ \\
\hline 15 & $\mathrm{Co}-57$ & $2.97 \mathrm{E}-08$ & 57 & I - 132 & $8.42 \mathrm{E}-05$ \\
\hline 16 & $\mathrm{Co}-58$ & $1.12 E-07$ & 58 & $I-133+D$ & $9.25 \mathrm{E}-06$ \\
\hline 17 & $\mathrm{Co}-60$ & $4.17 E-09$ & 59 & $\mathrm{I}-134$ & $2.20 \mathrm{E}-04$ \\
\hline 18 & $\mathrm{Ni}-57$ & $5.35 \mathrm{E}-06$ & 60 & $I-135+D$ & $2.92 \mathrm{E}-05$ \\
\hline 19 & $\mathrm{Ni}-63$ & $2.20 E-10$ & 61 & $\mathrm{Xe}-131 \mathrm{M}$ & $6.69 \mathrm{E}-07$ \\
\hline 20 & $\mathrm{Ni}-65$ & $7.64 \mathrm{E}-05$ & 62 & $X e-133 M$ & $3.61 \mathrm{E}-06$ \\
\hline 21 & $\mathrm{Cu}-64$ & $1.52 E-05$ & 63 & Xe-133 & $1.52 \mathrm{E}-06$ \\
\hline 22 & $Z n-65$ & $3.31 E-08$ & 64 & $\mathrm{Xe}-135 \mathrm{M}$ & $7.56 \mathrm{E}-04$ \\
\hline 23 & $Z n-69 M+D$ & $1.39 \mathrm{E}-05$ & 65 & $\mathrm{Xe}-135$ & $2.10 E-05$ \\
\hline 24 & As -76 & $7.32 \mathrm{E}-06$ & 66 & xe-137 & $3.01 \mathrm{E}-03$ \\
\hline 25 & $8 r-82$ & $5.44 E-06$ & 67 & $\mathrm{Xe}-138+\mathrm{D}$ & $8.14 \mathrm{E}-04$ \\
\hline 26 & $\mathrm{Kr}-83 \mathrm{M}$ & $1.04 \mathrm{E}-04$ & 68 & Cs-134 & $1.07 \mathrm{E}-08$ \\
\hline 27 & $\mathrm{Kr}-85 \mathrm{M}$ & $4.31 E-05$ & 69 & $C s-136$ & $6.17 \mathrm{E}-07$ \\
\hline 28 & $\mathrm{Kr}-85$ & $2.05 E-09$ & 70 & $C s-137+D$ & $7.31 \mathrm{E}-10$ \\
\hline 29 & $\mathrm{Kr}-87$ & $1.52 \mathrm{E}-04$ & 71 & Cs-138 & $3.58 \mathrm{E}-04$ \\
\hline 30 & $\mathrm{Kr}-88+\mathrm{D}$ & $6.89 \mathrm{E}-05$ & 72 & Cs $-139+D$ & $1.24 E-03$ \\
\hline 31 & $\mathrm{Kr}-89$ & $3.64 E-03$ & 73 & $\mathrm{Ba}-139$ & $1.39 \mathrm{E}-04$ \\
\hline 32 & $\mathrm{Rb}-88$ & $6.53 \mathrm{E}-04$ & 74 & $B a-140+D$ & $6.28 \mathrm{E}-07$ \\
\hline 33 & $R b-89+D$ & $7.61 \mathrm{E}-04$ & 75 & $\mathrm{La}-140$ & $4.78 \mathrm{E}-06$ \\
\hline 34 & $S r-89+0$ & $1.59 E-07$ & 76 & La-141 & $4.97 \mathrm{E}-05$ \\
\hline 35 & $S r-90+D$ & $7.58 \mathrm{E}-10$ & 77 & $\mathrm{Ce}-141$ & $2.47 \mathrm{E}-07$ \\
\hline 36 & $S r-91+D$ & $2.03 \mathrm{E}-05$ & 78 & $\mathrm{Ce}-144+\mathrm{D}$ & $2.83 E-08$ \\
\hline 37 & $5 r-92+D$ & $7.11 E-05$ & 79 & Eu-152 & $1.69 \mathrm{E}-09$ \\
\hline 38 & $Y-90$ & $3.01 \mathrm{E}-06$ & 80 & Eu-154 & $2.55 \mathrm{E}-09$ \\
\hline 39 & $Y-91 M+D$ & $2.32 E-04$ & 81 & W-187 & $8.06 \mathrm{E}-06$ \\
\hline 40 & $\mathrm{Zr}-95+\mathrm{D}$ & $1.22 E-07$ & 82 & Th $-232+D$ & $1.57 \mathrm{E}-18$ \\
\hline 41 & $2 r-97+0$ & $1.14 \mathrm{E}-05$ & 83 & $\mathrm{~Np}-239$ & $3.42 \mathrm{E}-06$ \\
\hline 42 & $\mathrm{Nb}-95$ & $2.29 \mathrm{E}-07$ & & & \\
\hline
\end{tabular}


TABLE A-4. Radius Intervals and Midpoints for Airborne Dose Calculations $(\mathrm{km})$

\begin{tabular}{cc} 
Interval & Midpoint \\
\hline $2-3$ & 2.5 \\
$3-4$ & 3.5 \\
$4-6$ & 5 \\
$6-9$ & 7.5 \\
$9-14$ & 11.5 \\
$14-20$ & 17 \\
$20-30$ & 25 \\
$30-40$ & 35 \\
$40-60$ & 50 \\
$60-80$ & 70
\end{tabular}

The $X_{0 Q D 0 Q}$ program generates four sets of atmospheric transport factors:

- average annual atmospheric dilution factors which are not corrected for cloud depletion or radioactive decay

- dilution factors which are only corrected for decay assuming a 2.26-day half-life

- dilution factors which are corrected for depletion and for decay assuming an 8-day half-life

- relative deposition per unit area.

These factors were used to estimate the dose from airborne releases using methods similar to the NRC GASPAR program (Eckerman 1976). Except for the two additional sites, the transport factors used this year were the same as those used for the 1976 estimates. The assumptions used in the calculation of these transport factors were as follows:

- 50-m source height with no correction for plume rise or building wake effects

- semi-infinite cloud model with sector-average, Gaussian-plume dispersion

- no correction for terrain height variation.

Since information about height and locations at each site for the releases given in Decker (1979) was unavailable, a single generic height of $50 \mathrm{~m}$ was used at each site for the release point. Because the heights and locations of releases are uncertain, estimates of dose to persons living within $2 \mathrm{~km}$ of the site could be in serious error; only persons living between 2 to $80 \mathrm{~km}$ from the site were included in the dose estimates. 
POPULATION

The population distribution within 2 to $80 \mathrm{~km}$ around each site was determined by using a computer program and data base derived from the 1970 census. The program and data base were developed by the Department of Commerce and subsequently adopted by the Environmental Protection Agency (EPA) for use with population exposure problems (Athey, Tell and Janis 1974, Hill 1977).

The population data base used was an edited and compressed version of the 1970 Census Bureau's Master Enumeration District List with Coordinates. It contains housing and population counts for each census enumeration district and the geographic coordinates of the population centroid for each district. Using a modified version of the EPA program and the data base, the population in each of the 160 segments around each site was estimated from a distance of 2 to $80 \mathrm{~km}$. The populations for 1977 were estimated using the "net increase" factors by state over the population values for 1970 as given in Statistical Abstract of the United States, 1978 (Table 12).

\section{FOOD PRODUCTION VERSUS FOOD CONSUMPTION}

The total food production for the region within $80 \mathrm{~km}$ around each site was the product of the NRC state-wide productivity figure for each state and a site productivity factor. At some sites this total production may be more or less than the total consumption; i.e., population times average individual consumption (see Table A-1 for generic consumption rates). When production was more than consumption for a site, it was assumed that all persons in the 2-to-80-km region ate contaminated food; when production was less than consumption, it was assumed that dilution would occur because uncontaminated food would be shipped into the area from outside. Thus, the calculated doses for a particular food type were reduced in proportion to the ratio of production $\div$ consumption (production/consumption < 1 ).

The dose to persons outside the 80-km 1 imit from food shipped out of the region, in the case of production being greater than consumption, is not included in this report because it is concerned only with the dose within the 80-km radius. These production/consumption factors are given as footnotes to the tables showing airborne dose commitment in the Site Summary section for reference.

\section{ORINKING WATER}

The population between 2 and $80 \mathrm{~km}$ of each plant site exposed to drinking water contaminated with released radionuclides was generally obtained from the environmental statement (ES) for the plant. For all sites located on salt water, it was assumed that no dose was received from drinking water. The consumption rates used are given in Table A-1 for drinking water. 
The radionuclide concentration in the drinking water consumed by a population downstream from a site was usually estimated assuming $100 \%$ mixing of the plant effluent with the river. For lakes, an overall dilution factor was est imated from dilution factors given in the ES for each population center along the shore (within $80 \mathrm{~km}$ ) which consume the contaminated lake water. These individual factors were weighted by population and averaged to obtain an effective dilution factor for the total population exposed to contaminated drinking water.

\section{AQUATIC FOOD}

Wherever possible, the fish-catch data from the plant ES was used to estimate aquatic food consumption rates for the population in the region. When this data was not found in the ES or was considered unrealistic, the generic values of Table A-1 were used.

The average radionuclide concentration of the waters in which this food was harvested was estimated assuming an additional dilution over the effluent flow from the reactor. For rivers, it was assumed that the fish were caught in waters in which the plant effiuent was completely diluted. For lakes, an additional factor as given in the ES was used; when none was given in the ES, a generic value of 0.01 was used. For ocean and bay sites, a generic value of 0.001 and 0.002 was used for fish and invertebrates, respectively, if the ES yielded no values for these parameters. Invertebrates were not assumed to be caught in sufficient quantity at freshwater sites (river and lake) to affect the population dose and therefore were not included in the dose calculation. Any exceptions to these general guidelines are explained in the footnotes to the individual site sumaries. 


\section{APPENDIX REFERENCES}

Athey, T. W., R. A. Tell and D. E. Janis. 1974. The Use of an Automated Population Data Base in Population Exposure Calculation, CONF 741018. Proceedings of Symposium on Population Exposures. NTIS, Springfield, VA.

Decker, T. R. 1979. Radioactive Materials Released from Nuclear Power Plants; (1977), NUREG-0521. U.S. Nuclear Regulatory Comission, Washington, DC. *

Eckerman, K. 1976. GASPAR DOSE CODE (Computer Listing). U.S. Nuclear Regulatory Commission, Washington, DC.

Hill, E. R. 1977. Calculation of Population Distribution Using a Computer Data Base, BNWL-2395. Pacific Northwest Laboratory, Richland, WA.

Hoenes, G. R., and J. K. Soldat. 1977. Age-Specific Radiation Dose Commitment Factors for a One-Year Chronic Intake, NUREG-0172. U.S. Nuclear ReguTatory Comission, Washington, DC.*

Population Estimates and Projections, Current Population Reports. 1975. Series P-25, No. 541. U.S. Department of Commerce, Social and Economic Statistics Administration, Bureau of the Census.

Regulatory Guide 1.109. 1977. Calculation of Annual Doses to Man from Routine Releases of Reactor Effluents for the Purpose of Evaluating Compliance with 10 CFR Part 50, Appendix I. Revision 1. U.S. Nuclear Regulatory Commission, Washington, DC.

Sagendorf, J. F., and J. T. Goll. 1977. XOQDOQ--Program for the Meteorological Evaluation of Routine Effluent Releases at Nuclear Power Stations, NUREG-0324. U.S. Nuclear Regulatory Commission, Washington, DC. *ᄎ

Statistical Abstract of the United States: 1978. 99th edition. U.S. Department of Commerce, Bureau of the Census, Washington, DC.

ॠAvailable for purchase from the NRC/GPO Sales Program, U.S. Nuclear Regulatory Commission, Washington, DC 20555, and the Nationa\} Technical Information Service, Springfield, VA 22161.

**Available free upon written request to the Division of Technical Information and Document Control, U.S. Nuclear Regulatory Commission, Washington, DC 20555. 

No. of

Copies

1 AA Churm

DOE Patent Division

$9800 \mathrm{~S}$. Cass Avenue

Argonne, IL 60439

2 DOE Technical Information Center

Oak Ridge, TN 37830

20 MR Beebe

Office of Management \& Program Analysis

Nuclear Regulatory Commission

Washington, DC 20555

50 Pacific Northwest Laboratory

P.0. Box 999

Richland, WA 99352

DA Baker (41)

BE Vaughan

EC Watson

Publishing Coordinator (2)

Technical Information (5) 


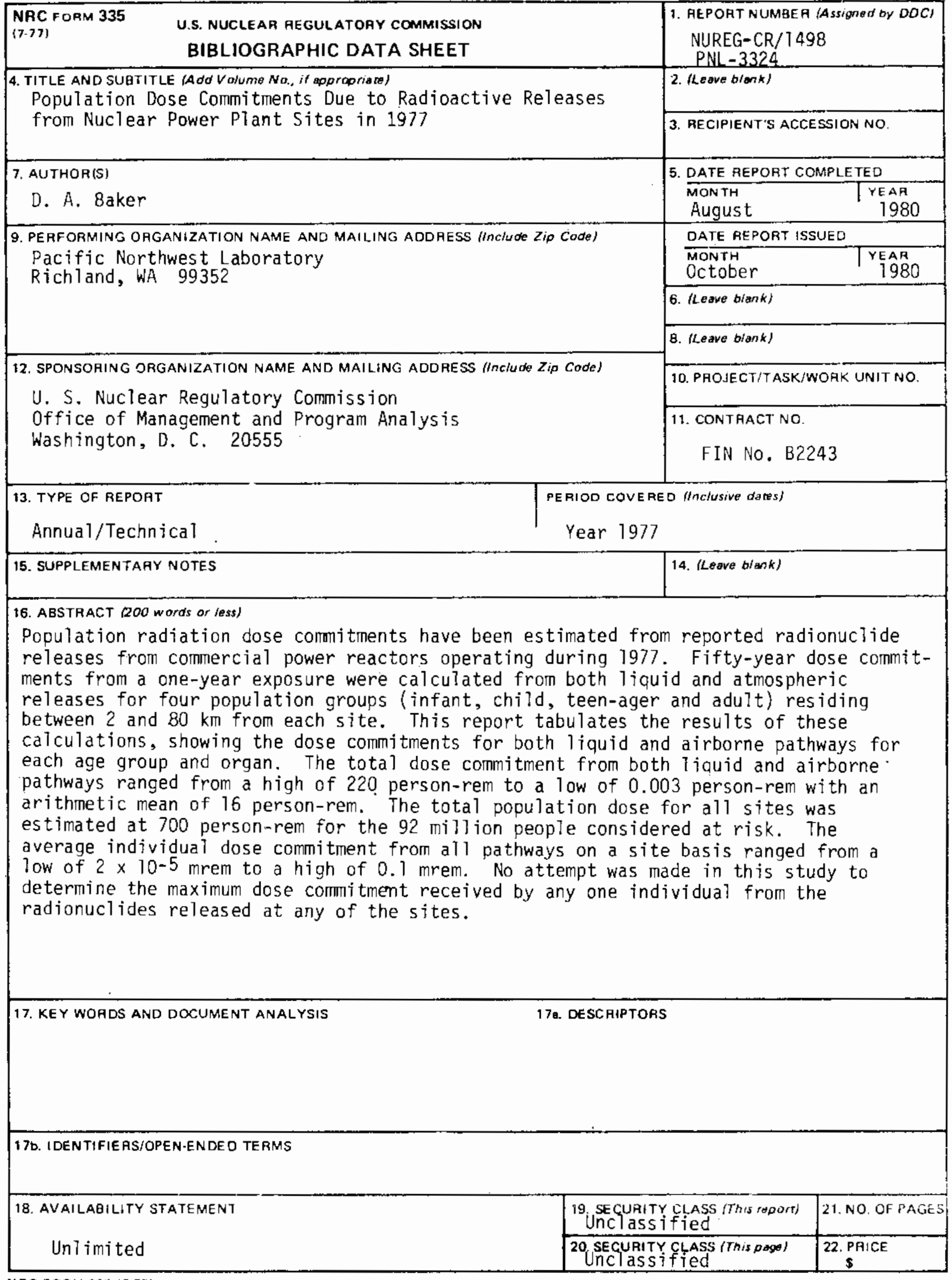


